\title{
How developmental and behavioural plasticity in the fall field cricket is influenced by the acoustic social environment and anthropogenic noise
}

\author{
by \\ Genevieve L. Ferguson
}

A thesis submitted to the Faculty of Graduate and Postdoctoral Affairs in partial fulfillment of the requirements for the degree of

Doctor of Philosophy
in

Biology

Carleton University

Ottawa, Ontario

(C) 2018, Genevieve L. Ferguson 


\section{Abstract}

The social environment is an important driver of developmental plasticity in juveniles and behavioural plasticity in adults. An individual's ability to accurately assess cues during development and predict its future social dynamics plays an important role in ensuring that its phenotype at adulthood will match their anticipated environment. However, because the overall plasticity of an individual is the result of a concomitant interaction between development, environment, and behaviour over the entire lifetime, constraints arising from development may hinder adult behaviour. My dissertation examines the influence of the developmental social environment on life-history traits and fitness-conferring adult behaviours to enhance our understanding of how these two life phases interact in the fall field cricket Gryllus pennsylvanicus. Through play-back experiments that altered the density of adult male acoustic signals, my findings reveal that male and female development time decreases and female residual mass at adulthood increases in higher perceived population densities. Contrary to my expectations, neither adult male aggressive nor mate attraction signalling were significantly influenced by developmental social environments. Adult female mate preference behaviour was influenced by developmental social environment, as females raised in social isolation were more responsive than females raised exposed to signals. Furthermore, the acoustic social environment experienced during development had significant indirect effects on all adult behaviours through constraints imposed on adult body size. Crickets reared in the high density environments developed faster, and a faster development time resulted in larger body sizes. Because body size significantly influenced male aggression and signalling behaviour, and female mate preference behaviour, the ability to express 
behavioural plasticity at adulthood may have been limited. However, my experiment that switched acoustic social environments between juvenile and adult stages showed that males who experienced a switched environment were able to adjust specific aspects of their signalling to match the unanticipated adult social dynamics. I also examined whether anthropogenic noise pollutes the bioacoustics network. Anthropogenic noise influenced male development time and signalling behaviour, and female residual mass and preference functions. Further, anthropogenic noise sometimes interacted with the acoustic social environment experienced during rearing, causing individuals to be mismatched with their social environment in adulthood. 


\section{Acknowledgements}

I am immensely grateful to my supervisor Dr. Susan Bertram, for her encouragement, support, guidance, and massive patience for my shenanigans over the past seven years. She has been an amazing mentor in academia and life. I thank her for the enormous contribution to my research, the completion of this thesis, her encouragement and support of my teaching, and her beer. Her enthusiasm, reassurance, as well as her friendship kept me going when things seemed impossible.

I am extremely grateful to the staff and faculty at Carleton University for their support during my doctoral studies. I would especially like to thank the Biology department's amazing support staff for their ability to solve administrative and greenhouse problems, and for helping me find and move (and find and move and find and move) a home for my experiments. I would also like to thank Drs. Jean-Guy Godin and Julie Morand-Ferron for serving on my thesis advisory committee, and for their thoughtprovoking questions, helpful advice, and encouragement over these past several years.

Also, a giant thank-you to all of the Bertram lab members both past and present for their support with animal care, experimental brainstorming, and assistance, including Maria Doria, Mykell Reifer, Emily Missyabit McAuley, Amy Villarreal, Michelle Loranger, Ian Thomson, Caitlin Grant, Lauren Fitzsimmons, as well as countless undergraduate students. I want to especially thank lab members Donovan Tremblay (for all the effort he put into keeping my crickets alive and the trials he ran) and to Michelle Leveillee (who spent so many hours watching crickets fight). I also want to acknowledge my 'lab wife' Sarah Harrison - without whom I never would have made it through the 
past years. Her kindness, friendship, and guidance have kept me sane, and the sushi we ate kept me happy.

The completion of this doctoral thesis would not have been possible without the support of my family and friends. A giant sloppy hug and thank you to my partner, James Jacques, for being an unending source of support, for taking care of me, cheering me on, providing the coffee, and occasionally doing the dishes. Thanks to my parents, John and Pat, for always encouraging me to go after my dreams, supporting me the whole time, and for always being in my corner. I love you Mom and wish you had been able to see me finish. I also never would have completed my thesis without the support of my sister Danielle, brother CodyAllan, and brother-in-law Matt (who has done so many edits I've lost count). As well my friends Gosia Kozak and Nicole Cote - who have pushed me when needed and held my hand the rest of the time. To everyone else who had to listen to me rant about crickets for seven years and still respond to my texts (Allee Holland, Melissa Wallace, Christine Halling, and Amy Harris), you are amazing and have my thanks.

Funding for the research presented in this thesis was provided through research grants awarded to my supervisor, Dr. Susan M. Bertram, from the Natural Sciences and Engineering Research Council of Canada Discovery Grants, the Canadian Foundation for Innovation, the Ontario Research Fund, and the Carleton University Research Fund. I was directly supported by Departmental Scholarships from Carleton University, an Animal Behavior Society Research Grant, the Hamlin Graduate Bursary, and the Lorraine Cinkant Bursary in Science. 


\section{Table of Contents}

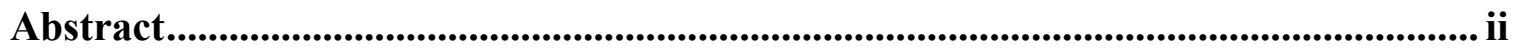

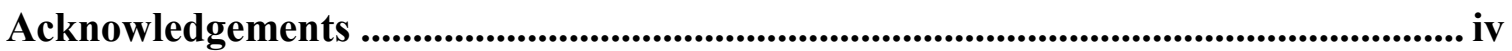

Table of Contents ............................................................................................................ vi

List of Tables ....................................................................................................................... xi

List of Figures...................................................................................................................... xvi

List of Appendices......................................................................................................... xxii

Chapter 1: General Introduction .............................................................................................. 1

1.1 Life History Traits and Phenotypic Plasticity ……………............................... 1

1.2 Developmental versus Behavioural Plasticity ................................................. 2

$1.3 \quad$ Social Environment and Plasticity ……………......................................... 4

$1.4 \quad$ The Importance of Juvenile Social Environment.............................................. 5

$1.5 \quad$ Use of Acoustics in Social Environment Studies ........................................... 6

$1.6 \quad$ Impact of Anthropogenic Noise ................................................................. 7

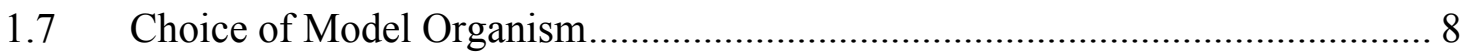

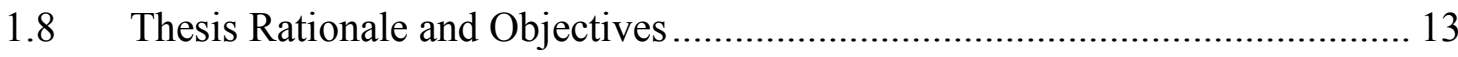

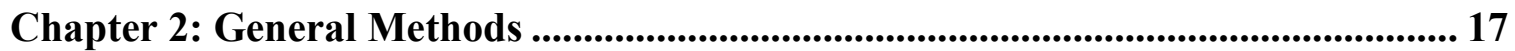

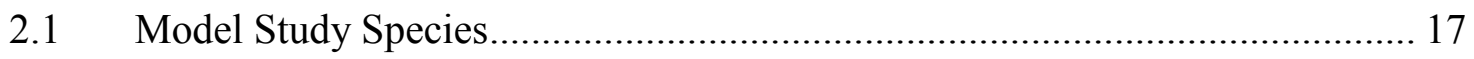

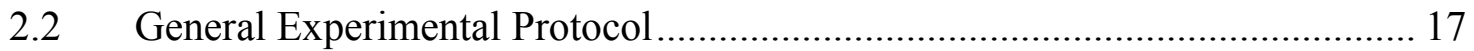

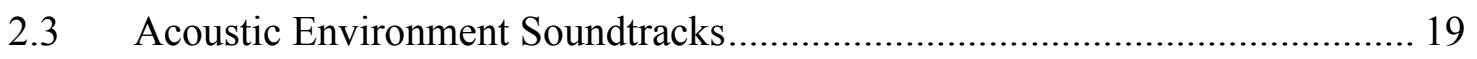

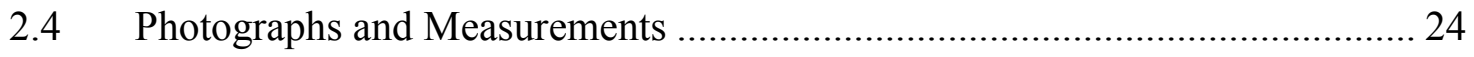

Chapter 3: Developmental acoustic social environment and anthropogenic noise affects field cricket expression of life history traits ......................................................... 26

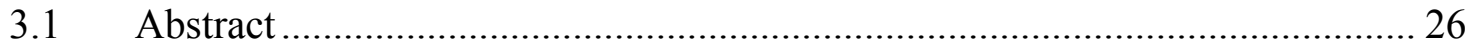




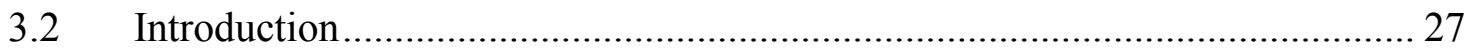

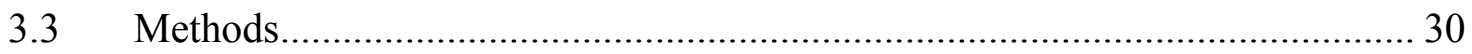

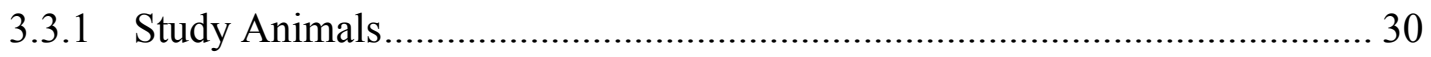

3.3.2 General Experimental Protocol ................................................................ 30

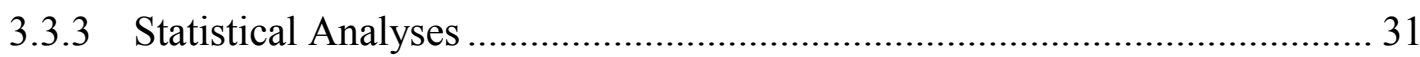

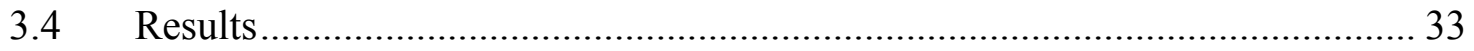

3.4.1 Impact of Acoustic Treatment on Female Developmental Traits ................. 34

3.4.2 Impact of Acoustic Treatment on Male Developmental Traits .................... 40

3.4.3 Impact of Development Time on Adult Male and Female Morphology ...... 44

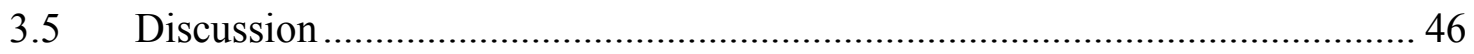

Chapter 4: Developmental acoustic social environment, but not anthropogenic noise, affects male field cricket aggression ..................................................................................... 53

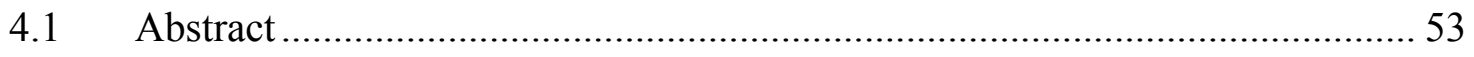

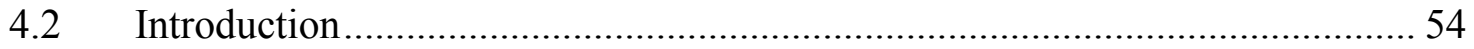

$4.3 \quad$ Methods

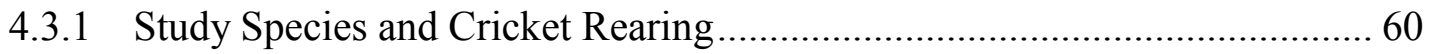

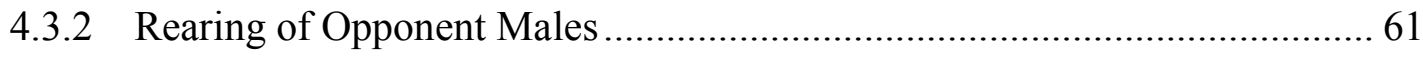

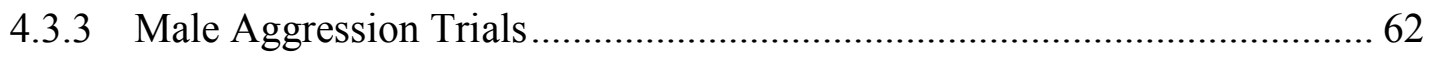

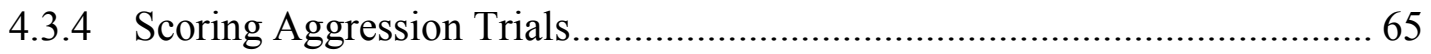

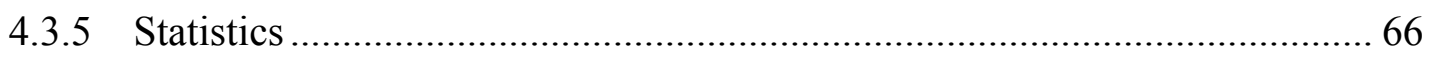

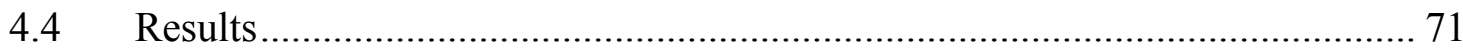

4.4.1 Impact of Acoustic Treatment on Male Aggressive Behaviour..................... 71

4.4.2 Impact of Acoustic social environment Treatment on Life History Traits ... 80

4.4.3 Impact of Development Time on Adult Body Size …................................... 84 


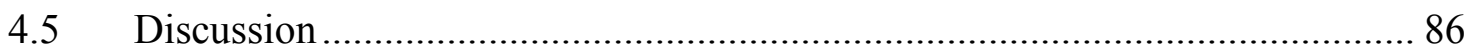

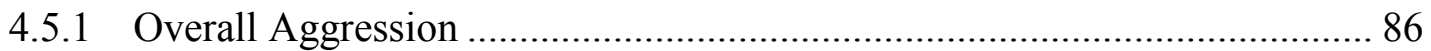

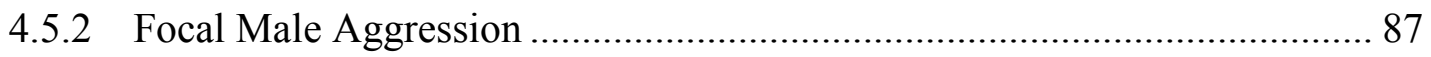

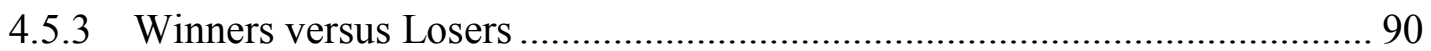

Chapter 5: Field cricket signalling behaviour is not influenced by the developmental acoustic social environment or anthropogenic noise ................................................... 93

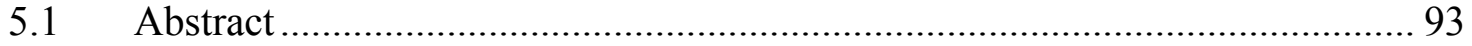

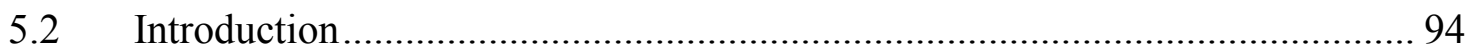

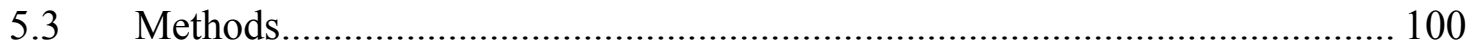

5.3.1 Study Species and Cricket Rearing ........................................................ 100

5.3.2 Adult Male Signalling Behaviour ................................................................ 101

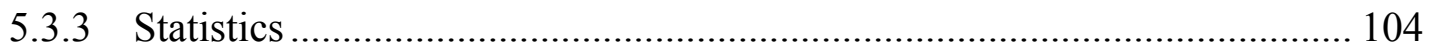

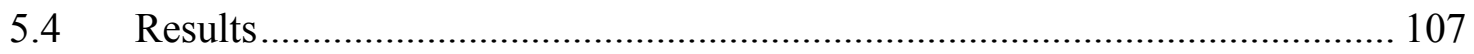

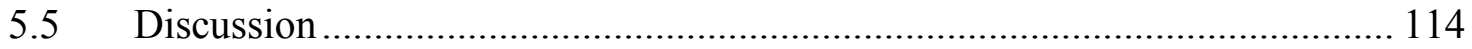

Chapter 6: Female field crickets that experience acoustic social signals during development expect more from a mate .................................................................................. 120

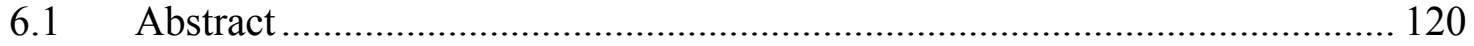

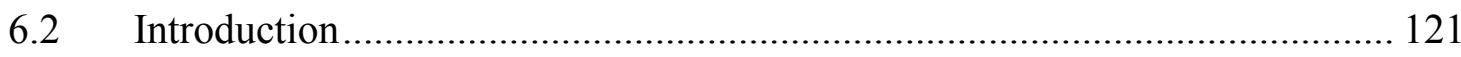

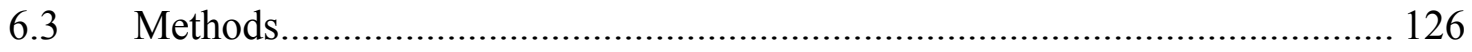

6.3.1 Study Species and Cricket Rearing......................................................... 126

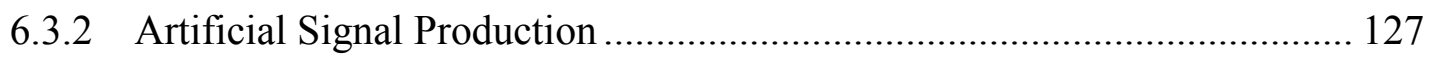

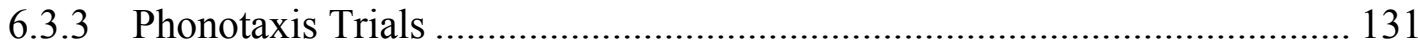

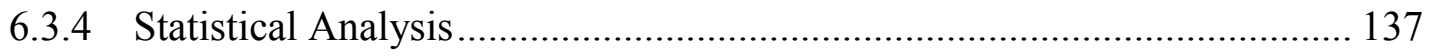

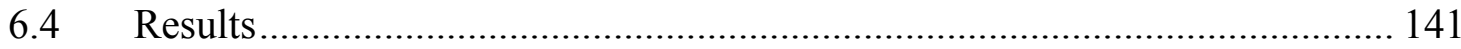


6.4.1 Preference Function Shape …………………........................................ 141

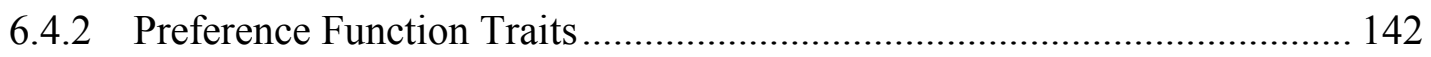

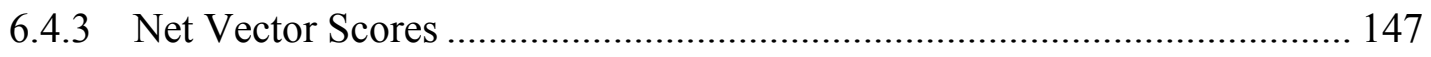

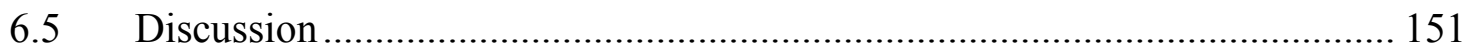

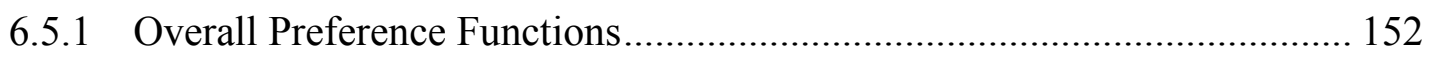

6.5.2 Impact of Acoustic Social Environment on Adult Female Preference ....... 152

6.5.3 Impact of Anthropogenic Noise on Adult Female Preference .................... 154

6.5.4 Impact of Life History Trait Variation on Adult Female Preference........... 155

\section{Chapter 7: Can adult male field crickets adjust their signalling behaviour when} presented with new social densities? ................................................................................. 157

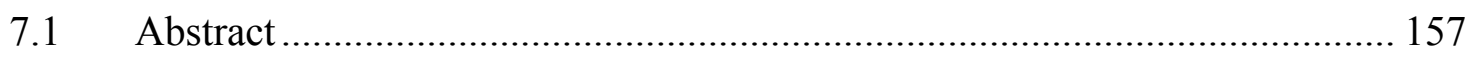

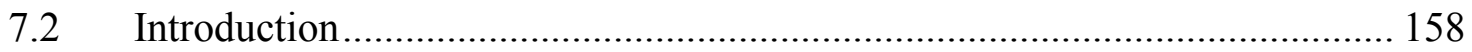

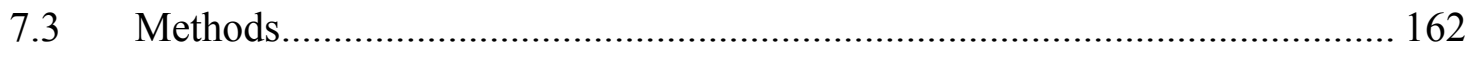

7.3.1 Study Species and Cricket Rearing ........................................................... 162

7.3.2 Adult Male Signalling Behaviour ………………………………….......... 163

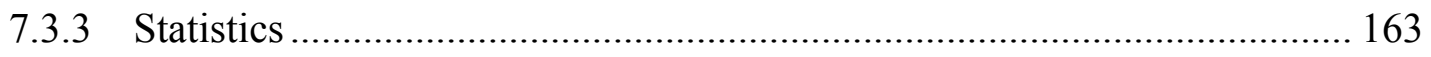

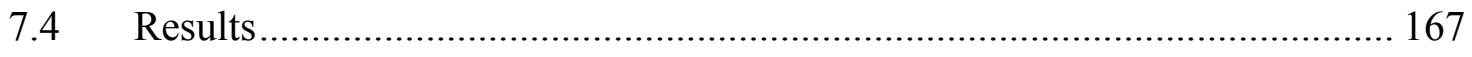

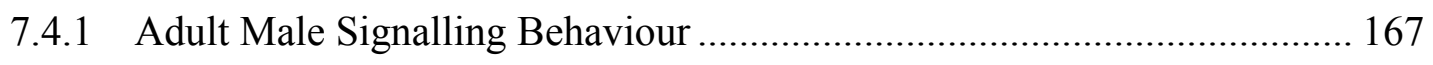

7.4.2 Impact of Acoustic Treatments on Life History Traits ............................... 174

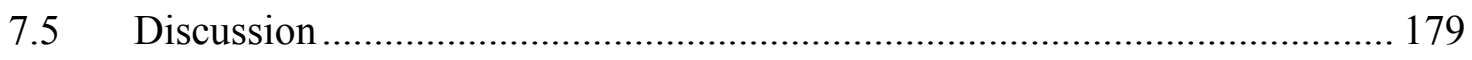

Chapter 8: General Conclusions................................................................................... 184

8.1 Influence of Juvenile Acoustic Social Environment on Life History Traits... 184

8.2 Influence of Juvenile Acoustic Social Environment on Adult Behaviour...... 185

8.3 Influence of Anthropogenic Noise ............................................................. 190 
8.4 Limitations and Future Studies

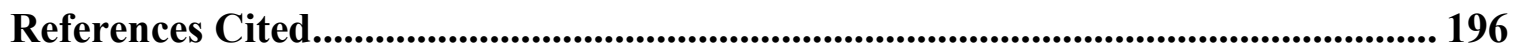

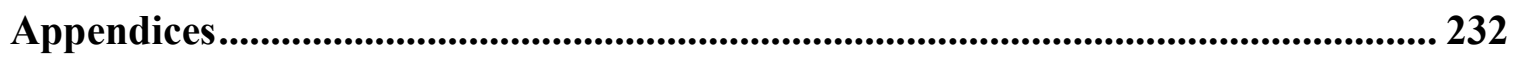

Appendix 1: Parameter Estimate Tables for Chapter 3 ....................................... 232

Appendix 2: Parameter Estimate Tables for Chapter 4 .......................................... 238

Appendix 3: Developmental Plasticity for Males from Chapter 4 and 5................. 245

Appendix 4: Parameter Estimate Table for Chapter 5 ........................................... 246

Appendix 5: Parameter Estimate Table for Chapter 6 ......................................... 249

Appendix 6: Parameter Estimate Table for Chapter 7 ........................................ 251 


\section{List of Tables}

Table 2.1. Acoustic environment treatments. Juveniles were raised in one of six acoustic treatments that represent differing levels of adult male calling (signalling density) and anthropogenic noise

Table 3.1. Results of the linear models examining effects of acoustic treatment on development time measures, adult body size (PC1 size) and adult residual mass in females. Significant effects are bolded for both models and model effects, FDR $\mathrm{B}_{\mathrm{Y}}$ corrected $P=0.02(\mathrm{~N}=285)$. Parameter estimates are included in Appendix 1, Table

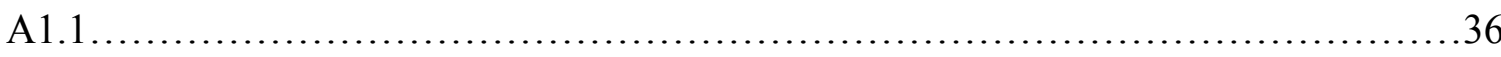

Table 3.2. Results of the linear models examining effects of juvenile acoustic treatment during development on development time, body size (PC1 size) and residual mass at adulthood in males. Significant effects are bolded for both models and model effects, FDR $_{\mathrm{B}-\mathrm{Y}}$ corrected $P=0.02(\mathrm{~N}=228)$. Parameter effects are included in Appendix 1,

Table A1.2 .............................................................. 41

Table 3.3. Results of the linear models examining effects of development time on body size $(\mathrm{PC} 1 \mathrm{size})$ and residual mass at adulthood in females $(\mathrm{N}=285)$ males $(\mathrm{N}=228)$. Significant effects are bolded for both models and model effects, FDR B-Y $_{\text {corrected }} P=$ 0.027. Parameter effects are included in Appendix 1, Table A1.3.....................45

Table 4.1. PCA factor loading values for aggression scores. All loadings were positively associated with the aggression factors 
Table 4.2. Results of the linear model examining effects of acoustic treatment, weight (mg) of males before the trial, and subject of male (focal vs opponent) on aggression levels. Significant effects are bolded. FDR $\mathrm{BY}_{\mathrm{B}}$ corrected level of significance is $P=0.0219$ $(\mathrm{N}=394)$. Parameter effects are included in Appendix 1, Table A2.1....................73

Table 4.3. Results of the generalized linear model on focal males examining effects of acoustic treatment, percentage difference in body weight between fighting males, body size of focal males (PC1 size), residual mass of focal males, and season on likelihood of being a winner or loser. Significant effects are bolded. FDR $\mathrm{BY}_{\mathrm{B}}$ corrected level of significance is $P=0.0193(\mathrm{~N}=197)$. Parameter effects are included in Appendix 1, Table A2.2

Table 4.4. Results of the linear models on focal males examining effects of acoustic treatment, percentage difference in body weight between fighting males, body size of focal males ( $\mathrm{PC} 1$ size), and season on aggression ( $\mathrm{PC} 1$ aggression). Significant effects are bolded for model effects. FDR $\mathrm{BY}_{\mathrm{BY}}$ corrected level of significance is $P=0.0193$ $(\mathrm{N}=197)$. Parameter effects are included in Appendix 1, Table A2.3

Table 4.5. Results of the linear models examining effects of acoustic treatment, percentage difference in body weight between trial partners, body size of focal males (PC1 size), and season on aggression (PC1 aggression) in focal males for losers and winners. Significant effects are bolded. FDR $\mathrm{BY}_{\mathrm{Y}}$ corrected level of significance is $\mathrm{P}$ $=0.0193\left(\mathrm{~N}_{\text {losers }}=67, \mathrm{~N}_{\text {winners }}=130\right)$. Parameter effects are included in Appendix 1, Table A2.4. .78 
Table 4.6: Results of the linear models examining effects of acoustic treatment and season on development time, adult body size (PC1 size), residual mass at adulthood. Significant effects are bolded for both models and model effects. FDR $\mathrm{BY}_{\mathrm{BY}}$ corrected level of significance is $P=0.024(\mathrm{~N}=350)$. Parameter effects are included in Appendix 1,

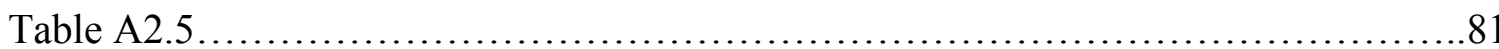

Table 4.7. Results of the linear models examining effects of development time on body size $(\mathrm{PC} 1$ size $)$ and residual mass at adulthood in males $(\mathrm{N}=350)$. Significant effects are bolded for both models and model effects, FDR $\mathrm{BY}_{\mathrm{B}}$ corrected $P=0.027$. Parameter effects are included in Appendix 1, Table A2.6....................................... 85

Table 5.1. Rotated PCA factor loading values for signal factor 1 and 2 of 8 mean daily fine scale signalling compoents after varimax roation. A negative sign (-) on the loading indicates that higher levels of that factor are negatively associated with that signalling parameter. Loadings above 0.600 are shown in bold 106

Table 5.2. Linear model effects examining how acoustic social environment treatment, body size, residual mass, participation in an aggression trial, and season influence time spent signalling, amplitude, and signal factors 1 and 2. Significant effects are bolded. FDR $_{\mathrm{BY}}$ corrected level of significance is $P=0.02$. Parameter estimates are included in Appendix 4, Table A4.1................................................. 109

Table 6.1. Mean and standard deviation values for long-distance mate attraction signal parameters of the sample population used to create the artificial signals 
Table 6.2: Rotated PCA factor loading values for preference factor 1 and 2 of four preference traits after varimax roation. Signs $(+/-)$ on loadings indicate that higher levels of a factor are positively or negatively associated with that parameter. Loadings above 0.600 are shown in bold

Table 6.3. Model effects of the generalized linear model examining effects of acoustic treatment, residual mass, adult body size (PC1 Size), and season on female preference

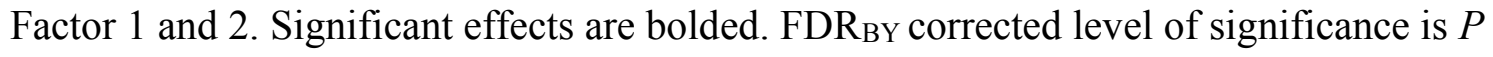
$=0.022$. Parameter estimates are included in Appendix 5, Table A5.1...

Table 6.4. Model effects of the generalized linear model examining the effects of trial order, adult body size (PC1 size), residual mass, season, acoustic treatment, and chirp rate on female net vector scores. Significant effects are bolded. FDR $\mathrm{BY}_{\mathrm{BY}}$ corrected level of significance is $P=0.015$. Parameter estimates are included in Appendix 5, Table

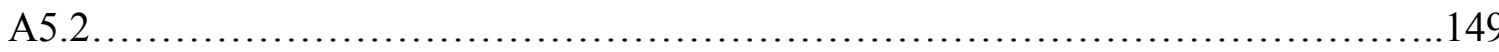

Table 7.1. PCA factor loading values for signal factor 1,2 and 3 of 8 mean daily fine scale signalling compoents after varimax roation. A negative sign (-) on the loading indicates that higher levels of that factor are negatively associated with that signalling parameter. Loadings above 0.600 are shown in bold 166 
Table 7.2. Results of the linear models examining effects of juvenile and adult acoustic treatment, adult body size, residual mass at adulthood and before the signalling trial, and season on signal factors 1, 2 and 3, time spent signalling, and amplitude. Significant

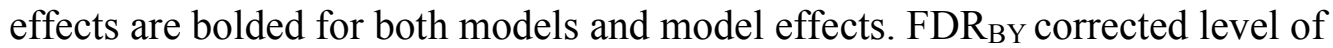
significance is $P=0.018(\mathrm{~N}=118)$. Parameter estimates are included in Appendix 6,

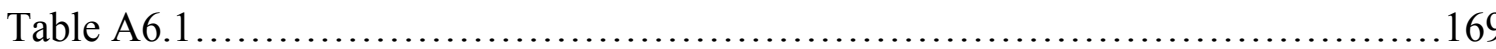

Table 7.3. Results of the linear models examining effects of acoustic treatment and season on development time, adult body size (PC1 size), residual mass at adulthood. Significant effects are bolded for both models and model effects. FDR $\mathrm{BY}_{\mathrm{B}}$ corrected level of significance is $P=0.033(\mathrm{~N}=124)$. Parameter estimates are included in Appendix 6, Table A6.2 ................................................................ 175 


\section{List of Figures}

Figure 1.1. A male field cricket with its forewings raised in signal production. The file on the right wing is brushed across the scraper on the left wing during the closing stroke of the wings, producing a single pulse of sound. Pulses are concatenated into chirps (e.g.,

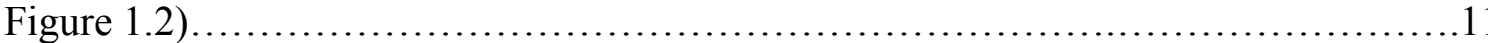

Figure 1.2. Waveform of 2 chirps from a G. pennsylvanicus long distance mate attraction signal displaying some of the various signal parameters I quantified 12

Figure 2.1. Stylized schematic representing how the environmental soundtracks were created. Shown above are samples from the Low density Soundtrack, consisting of signals from 3 separate males; and the High density Soundtrack, which consisted of the Low Soundtrack overlaid 4 times resulting in a total of 12 male signals. These signals were played across a 24-hour period that mimicked the natural temporal shifts in

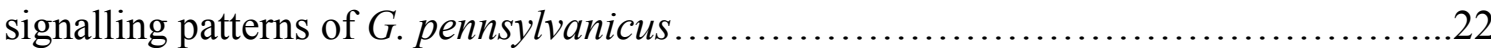

Figure 2.2. Schematic depicting the three standardized measurements used to determine adult body size in both male (pictured) and female crickets.........................25

Figure 3.1. How the acoustic social environment during development influences female development time across the different life stages: (A) Hatching to Wing pad, (B) Wing pad to adult eclosion, and (C) Total Development Time. Error bars represent standard errors and lowercase letters denote significant differences between acoustic treatment

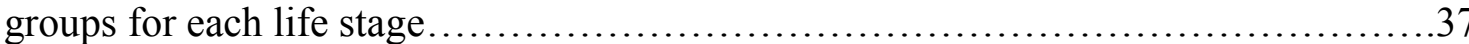


Figure 3.2. How the acoustic social environment during development influences female body size (PC1 size) at adult eclosion. Bars represent the average PC1 size and error bars are standard errors; letters denote significant differences........................... 38

Figure 3.3. How the interaction between anthropogenic noise and the acoustic social environment during development influences female residual mass at adult eclosion. Bars are standard errors and letters denote significant differences

Figure 3.4. How the acoustic social environment during development influences male development time across the different life stages: (A) Hatching to Wing pad, (B) Wing pad to adult eclosion, and (C) Total Development Time. Error bars are standard errors and lowercase letters denote significant differences between acoustic treatment groups for

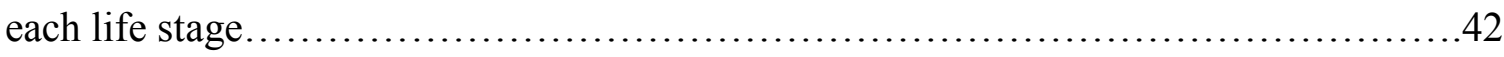

Figure 3.5. How the interaction between anthropogenic noise and the acoustic social environment during development influences male development time for Wing pad to adult eclosion. Bars are standard errors and letters denote significant differences........43

Figure 4.1. Experimental design set up showing the sound-attenuating chamber, trail arena, and GoPro HERO4 camera. During trials this chamber was closed, and trials were observed via the camera to limit disturbance of the males

Figure 4.2. Leverage residual plot showing the effects of adult body size (PC1 size) on aggression levels were negative values are less aggressive. The solid line indicates the relationship between body size and signalling component after all other variables in the model has been accounted for; the dashed lines represent $95 \%$ confidence intervals......76 
Figure 4.3. The effects of developmental acoustic treatment (Signalling Density) on Aggression levels in losing males. Negative values are less aggressive. Bars are standard errors and letters denote significant differences.

Figure 4.4. Effect of developmental acoustic treatment (signalling density) on development time (days) for the different life stages: (A) Hatching to Wing pad, (B) Wing pad to adult eclosion, and (C) Total Development Time. Error bars are standard errors and lowercase letters denote significant differences between acoustic treatment groups for each life stage................................................. 82

Figure 4.5. The interaction of signalling density and anthropogenic noise as it affects total development time (Days from Hatching to Adult eclosion). Bars are standard errors and letters denote significant differences. .83

Figure 4.6. Schematic highlighting the indirect effects that rearing acoustic environment can have on adult behaviours through steps of smaller, direct effects on different aspects of life history traits .89

Figure 5.1. Waveform of 2 chirps from a $G$. pennsylvanicus long distance mate attraction signal exhibiting some of the parameters that are recorded and measured by the EARS

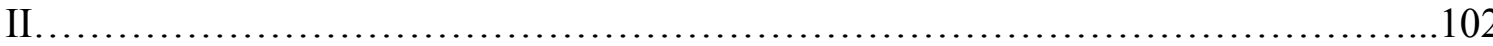

Figure 5.2. Effects of the interaction between anthropogenic noise and rearing environment density on male time spent signalling (mean daily mins) $(P=0.06)$

Figure 5.3. Effects of the interaction between anthropogenic noise and rearing environment density on male signalling amplitude (mean daily $\mathrm{dB})(P=0.05)$ 
Figure 5.4. Leverage residual plots showing how adult body size (PC1) influence signalling components. Larger males signalled with (A) more pulses per chirp with longer pulse durations and shorter interpulse durations as well as longer chirp durations (signal factor 1), (B) signalled more throughout the day (non-significant trend), and (C) signalled at higher amplitudes (db). Solid line indicates the relationship between body size and signalling component after all other variables in the model has been accounted for; the dashed lines represent $95 \%$ confidence intervals

Figure 5.5. Schematic highlighting the indirect effects that rearing acoustic environment can have on adult behaviours through steps of smaller, direct effects on different aspects of life history traits

Figure 6.1. Phonotaxis trial apparatus set-up detailing placement of speakers, lights, GoPro, and spherical treadmill within the sound attenuating container. Air tubes are observed exiting the container to an external air-compressor....

Figure 6.2. Detail of spherical treadmill within cowling with mounted female cricket. The design of the elastic hook apparatus on the female's pronotum allowed her to freely rotate and run on the treadmill with little to no resistance during phonotaxis trails.

Figure 6.3. Average preference functions (solid lines) with standard error bars for females who developed in each acoustic social environmental treatment, for A) different signalling densities without anthropogenic noise), and B) different signalling densities with anthropogenic noise, fitted with Pfunc. 
Figure 6.4. Marginal effect plots with 95\% confidence intervals showing how A) adult body size and B) signalling density (near-significant) during development effect responsiveness (Preference Factor 1) to the artificial signals

Figure 6.5. Marginal effect plots with 95\% confidence intervals showing how signalling density during development effect choosiness (Preference Factor 2) to the artificial signals (non-significant result) 146

Figure 6.6. Marginal effects plots with $95 \%$ confidence intervals showing how female preference (net vector score) was influenced by interactions between the standard deviation of chirp rate within the artificial signals and A) the signalling density during development, B) the presence of anthropogenic noise (near significant), and C) adult body size at the two extremes.............................................. 150

Figure 7.1. How the interaction between juvenile acoustic signalling density and adult acoustic signalling density affects signal factor 2 . Bars are standard errors

Figure 7.2. Leverage residual plot showing the effects of adult body size (PC1 size) on A) signal factor 1 (near significant), B) time spent signalling (min), and C) amplitude (db). Solid line indicates the relationship between body size and signalling component after all other variables in the model has been accounted for; the dashed lines represent $95 \%$ confidence intervals 
Figure 7.3. Leverage residual plot showing the effects of adult residual mass on signal factor 1 . Solid line indicates the relationship between body size and signalling component after all other variables in the model has been accounted for; the dashed lines represent $95 \%$ confidence intervals

Figure 7.4. Effect of developmental acoustic treatment (signalling density) on development time (days) for the different life stages: (A) Hatching to Wing pad, (B) Wing pad to adult eclosion, and (C) Total Development Time. Error bars are standard errors and lowercase letters denote significant differences between acoustic treatment groups for each life stage.................................................... 176

Figure 7.5. How the acoustic social environment during development influences male body size (PC1 size) at adult eclosion. Bars represent the average PC1 size and error bars are standard errors; letters denote significant differences............................177

Figure 7.6. How the acoustic social environment during development influences male residual mass at adult eclosion. Bars represent the average PC1 size and error bars are standard errors; letters denote significant differences........................... 178 


\section{List of Appendices}

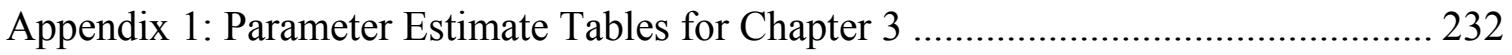

Appendix 2: Parameter Estimate Tables for Chapter 4 .......................................... 238

Appendix 3: Developmental Plasticity for Males from Chapter 4 and 5.................... 245

Appendix 4: Parameter Estimate Table for Chapter 5 ................................................ 246

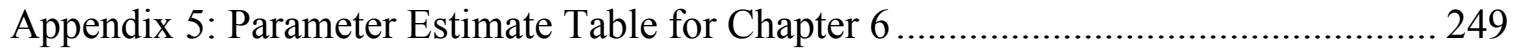

Appendix 6: Parameter Estimate Table for Chapter 7 ............................................. 2511 


\section{Chapter 1: General Introduction}

\subsection{Life History Traits and Phenotypic Plasticity}

The study of life history traits and their plasticity is a broad area of interest within the fields of both animal behaviour and evolutionary ecology. Life history traits of both individuals and groups can provide researchers with invaluable information concerning how changes in evolutionary and environmental factors shape development and behaviour. Life history traits are numerous, interconnected, and include growth rate (DiRienzo et al. 2012; Niehaus et al. 2012); development time (Anderson \& Kasumovic 2017); body size (Whitman 2008); adult mating behaviour and reproduction (Hunt et al. 2005); and survival or longevity (Doyon \& Boivin 2005). Because the heritability of life history traits is variable, their influence on adaptation can change across environments (Price \& Schluter 1991; Stearns 1980; Schluter et al. 1991; Sedinger et al. 1995). For example, differences in larval developmental patterns of the tree frog (Rama sylvatica) were reviewed by Berven \& Gill (1983) and found to be partially explained through heritability, but the amount of genetic influence over these traits varied between populations at different latitudes. Genetic influences were also found to affect variation of gosling growth and survival in the Black Brant (Branta bernicla nigricans), but later in the summer, gosling size was more influenced by date of hatching and the resulting environmental conditions (Sedinger et al. 1995). Changes in the environment can thus drastically alter the evolutionary trajectory of life history traits.

The relationship between environmental conditions and life history trait outcomes is widely understood as phenotypic plasticity, where the environment acts on a single genome to produce more than one adult phenotype (West-Eberhard 1989; Nylin \& 
Gotthard 1998; Dewitt et al. 1998; Agrawal 2001). Despite being recognized by Darwin as a characteristic which natural selection acts upon, phenotypic plasticity has often been overlooked as the focus of selection due to the aforementioned variation in heritability (West-Eberhard 1989; West-Eberhard 2005). However, the potential importance of phenotypic plasticity cannot be understated, as phenotypic plasticity can alter the traits available to selection, and thus genetic modification, by shifting the range of phenotypes expressed within a population. As such, gene frequencies can be modified through selection of plastic traits that are potentially non-genetic (West-Eberhard 1989; Pigliucci 2005; Price et al. 2003; Moczek et al. 2011; West-Eberhard 2005; Gilbert et al. 2015).

\subsection{Developmental versus Behavioural Plasticity}

Phenotypic plasticity of traits can be developmental or behavioural in nature (Kasumovic \& Brooks 2011). Developmental, or ontogenic plasticity, is a response to the environment that occurs before maturity and results in specific adult phenotypes that may or may not be permanent (Tremmel \& Muller 2013). To form a developmentally plastic response, environmental cues must occur early enough in ontogeny to trigger the appropriate adult phenotype. Triggered individuals can then modify their developmental trajectories to match the environmental contexts they are likely to face when older (Kasumovic \& Brooks 2011). Developmental plasticity often occurs in response to large-scale environmental variation, such as changes to photoperiod, temperature, or predator abundance (Adler \& Drew Harvell 1990; Nijhout 1999). For example, larvae of the Pacific tree frog (Pseudacris regilla) reared with exposure to different predator cues develop differences in both morphology and foraging behaviour (Bernard 2006). Furthermore, temperature during nymph development in the striped ground cricket 
(Allonemobius fasciatus) significantly influences adult male signalling behaviour as evidenced by changes in chirp rate, chirp duration, and carrier frequency (Olvido \& Mousseau 1995).

In contrast, behavioural plasticity, sometimes termed behavioural flexibility, is a response to fluctuations in the environment that can occur throughout an individual's life and often involves a large range of behavioural mechanisms (Bretman et al. 2011; Mery \& Burns 2010; Mousseau \& Roff 1989; Dingemanse et al. 2012). For example, plastic behaviour has been widely observed across taxa in response to sudden changes in available habitat and food/prey availability, leading to differences in how animals deposit eggs, forages, and hunt (Day \& McPhail 1996; Diaz-Fleischer \& Aluja 2003; Morse 1971). Critically, because behaviourally plastic traits can be flexible, and even reversible, the costs to the animal may be less than the cost of a developmentally plastic trait. These reduced costs allow for differences in resource allocation during development enabling individuals to be in better physical condition at maturation or to be better suited to the expected environment, incurring a benefit towards survival and increased fitness (Bretman et al. 2011; West-Eberhard 1989).

A key concern afflicting plasticity studies is their lack of inclusivity for the entire life-history of the individuals being examined. Research that focuses on how reversible behavioural plasticity allows individuals to adjust their behaviour in response to rapidly changing environments often fails to acknowledge that the success of any individual can still be limited by the phenotype at maturity (Kasumovic \& Brooks 2011). Conversely, for every morphology fixed at maturity, there may be several optional behaviours. The overall plasticity of an individual is therefore the result of a concomitant interaction 
between development, environment, and behaviour over the entire lifetime (WestEberhard 1989). Thus far, little empirical research has focused on the integrative phenotype created by the interaction of different life stages. To achieve this, we need to learn how behavioural plasticity is constrained by concomitant variation in developmental plasticity so that we can create a more complete understanding of how plasticity creates dynamic phenotypes for selection to act upon.

\subsection{Social Environment and Plasticity}

Individuals encounter and interact with a social milieu that acts as an important component of the environment and has the potential to affect plasticity. Social cues can change throughout an individual's lifetime and may differentially affect developmental and behavioural plasticity. Traits that partly depend on social interactions include growth and development, aggression, territoriality, and courtship and mate choice (Moore et al. 1997; Dingemanse \& Wolf 2013). For example, in the red deer (Cervus elaphus) changes in population densities affect survival, pregnancy rates, and body mass. In both males and females, high densities at birth results in smaller body mass in yearlings but not adults, higher adult density results in lighter adults, pregnancy rates in first breeding yearlings is lower at higher densities, and higher lifetime density results in lower male survival rates (Bonenfant et al. 2002). In male albino laboratory mice (Mus musculus) aggression levels are affected by whom they are housed with and when. Males housed with females are more aggressive than males housed with other males, and isolation during either juvenile or early adult life stages increases fighting tendencies (Crawley et al. 1975). Furthermore, male guppies (Poecilia reticulata) decrease their courtship displays as population densities increase, and male-male competition as well as mate searching is highest in 
intermediate population densities (Jirotkul 1999b). Population dynamics could thus potentially have strong effects on plastic traits and should therefore be considered in animal studies to understand their importance.

\subsection{The Importance of Juvenile Social Environment}

Emerging research suggests that the social environment experienced by juveniles during development is also important in determining adult traits. Juveniles can use cues of conspecifics to predict their future adult group dynamics and modify their development rate and resulting phenotype at maturity accordingly, ostensibly in an attempt to match expectant competition and mate availability (Kasumovic et al. 2009; DiRienzo et al. 2012). For example, developmental social isolation in the zebrafish (Danio rerio) affects both adult behaviour and brain function. Shams et al. (2017) found that long term social isolation leads to hyperactivity, disruption of shoaling, and a decrease in anxiety-like responses in adult zebrafish. Importantly, these behavioural changes were accompanied by decreased levels of a neurochemical (DOPAC) used in dopamine metabolism (Shams et al. 2017). Social isolation during development also affects adult foraging behaviour in female cactus bugs, (Chelinidea vittiger), where isolated females prefer to forage alone; in contrast females raised in groups prefer to forage in social groups (Miller et al. 2012). In the zebra finch (Taeniopygia guttata) larger brood sizes leads to faster development time, smaller adult size, and shorter lifespan in both wild and domesticated groups (Tschirren et al. 2009). Female wolf spiders (Schizocosa rovneri) use multiple cues during development to alter their preference as adults (Rutledge et al. 2010). Juvenile females exposed to differing visual and chemical male signals during development prefer to mate with unfamiliar male phenotypes as adults (Rutledge et al. 2010). Furthermore, 
altering exposure to acoustic sexual signals during development can alter both life history traits and adult sexual behaviour in several cricket species, including Gryllus integer (DiRienzo et al. 2012), Teleogryllus commodus (Kasumovic et al. 2011), and Teleogryllus oceanicus (Bailey et al. 2010). These studies revealed that juvenile crickets reared in the presence of conspecific sexual signals had a larger mass at adulthood (males), differential growth rates depending on signal quality and density (both sexes) and used acoustic signals to determine adult population density resulting in different resource allocation strategies and phenotypes during development (males). Thus, the acoustic environment can play an important role in development and needs to be considered when quantifying phenotypic plasticity and its potential impacts on fitness.

\subsection{Use of Acoustics in Social Environment Studies}

A major component of many animal social systems is acoustic communication, where one signal can contain multiple messages, including mate advertisement, aggressiveness, predator avoidance, species recognition, parental behaviour, foraging, or group coordination (Simmons 2003; McGregor \& Peake 2000). Acoustic communication is also open by nature, meaning that information contained in the signal can be transmitted beyond the intended receiver, and can thus affect many individuals simultaneously (Snijders \& Naguib 2017; Otte 1974). Eavesdroppers (unintended signal receivers) have the potential to alter their own life history trajectories during development or adult behaviour in response to the information contained in the signals they overhear (McGregor \& Peake 2000; Doutrelant \& McGregor 2000; Kasumovic \& Brooks 2011). Signallers can also be affected by eavesdropping if they are aware of the audience and modify their behvaiour accordingly (Montroy et al. 2016; McGregor \& Peake 2000). 
Additionally, from a research perspective, measuring and recording acoustic signals is relatively easy and unobtrusive, making bioacoustics an invaluable tool to analyze animal social networks.

\subsection{Impact of Anthropogenic Noise}

In addition to differences in the amount and quality of conspecifics in the rearing environment, other acoustic information present in the environment may also affect development. Of recent interest is how anthropogenic noise may alter the production and reception of acoustic signals of animals living near urban areas. Human habitation greatly alters the acoustic structure of the environment, resulting in a phenotypic change in the acoustic communication of many species living in the surrounding area (Pijanowski et al. 2011; Laiolo 2010). Signallers have the potential to adapt to urban noise by altering amplitude, frequency, or timing, as observed in birds and amphibians (Luther \& Derryberry 2012; Laiolo 2010). For example, an increase in both time spent signalling and amplitude, metabolic costly behaviours, is found in the suburban serin (Serinus serinus) when urban noise increases (Diaz et al. 2011). Further, male brown tree frogs (Litoria ewingii) increase both the pitch and frequency of their signals when anthropogenic noise is present (Parris et al. 2009). Anthropogenic noise can also affect the reception of signals, through either masking or interference. In the grey treefrog (Hyla chrysoscelis) for example, female phontotaxis towards a male signal is hindered in the presence of traffic noise (Bee \& Swanson 2007). Low-frequency signals of male great tits (Parus major), which are preferred by females, are masked in urban noise conditions, shifting female response towards higher frequency signals (Halfwerk et al. 2011). Anthropogenic noise therefore has the potential to alter animal behavioural patterns and 
should be considered when studying phenotypic plasticity of species that communicate acoustically in an urban environment.

Despite the known effects of the acoustic environment on invertebrates, very few studies have examined how anthropogenic noise impacts invertebrate communication systems. Emerging evidence suggests, however, that anthropogenic noise can interfere with both the transmission and reception of acoustic signals in grasshoppers (Chorthippus biguttulus, Lampe, Schmoll, Franzke, \& Reinhold, 2012), cicadas (Cryptotympana takasagona, Shieh, Liang, Chen, Loa, \& Liao, 2012), tree crickets (Oecanthus pellucens, Orci, Petr, and Barta 2016), and field crickets (Teleogryllus oceanicus, Gurule-Small \& Tinghitella, 2018; Gryllus bimaculatus, Schmidt, Morrison, \& Kunc, 2014). Like much of the current research on anthropogenic noise however, most of these studies are limited to changes in the acoustic communication of adults. They do not explore how exposure to anthropogenic noise during development influences developmental plasticity of juveniles or any subsequent adult behavioural plasticity.

\subsection{Choice of Model Organism}

While widespread across taxa, acoustic communication is especially pervasive amongst insects where hearing and broadcasting are used for predator avoidance, host localization, and reproduction (Pollack 2017). Insects have also evolved specialized physiology to ensure that, although physically small, their signals have large acoustic output. As such, insect signals are ripe for passing on information to eavesdroppers within (and beyond) their intended audience (Pollack 2017; Morley et al. 2013; Yager 1999). In particular, extensive research has been conducted on the auditory and acoustic systems of field crickets (Gryllidea) (Alexander 1962; Nocke 1972; Doherty \& Storz 1992; Souroukis et 
al. 1992). Not only are cricket signals one of the most complicated acoustic systems in invertebrates, they are also globally located, making them invaluable to evolutionary analysis (Alexander 1968; Alexander 1962).

I therefore used the North American fall field cricket, Gryllus pennsylvanicus, as my model study organism. Field crickets are sexually dimorphic with males producing acoustic signals using their forewings. These signals, a long-distance mate attraction signal, a quieter courtship song, and an aggression signal, are an integral part of the field cricket's complex mating system (Alexander 1961). Males produce acoustic signals by rubbing their forewings together; the left tegmen is modified into a scraper and the right tegmen is modified into a file, which scrape across each other as the wings are closed, releasing a pulse of sound (Walker 1962) (Figure 1.1). Pulses are concatenated into chirps and multiple chirps into signals which can vary between males (Figure 1.2). Long distance mate attraction signals are used by female crickets to locate and potentially differentiate between potential suitors. In general, female field crickets appear to prefer mate attraction signals that are more energetically expensive to produce, such as louder chirps and longer signalling bouts (Hoback \& Wagner 1997; Hedwig \& Poulet 2004; Hedrick 1986; Judge et al. 2014).

The fall field cricket is an ideal species to study the impact of how the acoustic social environment influences juvenile development, because fall field cricket acoustic signals can travel long distances and be heard by individuals other than intended receivers, such as juvenile conspecific eavesdroppers. Therefore, these acoustic signals have the potential to convey important information about the male composition of the current social cohort (e.g., density and male fitness) to juvenile conspecifics within 
hearing range (Otte 1974). The acoustic social environment also has the potential to influence several aspects of adult cricket behaviour such as male aggression and mate attraction signalling (Alexander 1961), and female mate choice (Souroukis \& Cade 1993; Souroukis \& Murray 1994). I therefore investigated how the density of acoustic sexual signals (the acoustic social environment) influenced juvenile development and adult mating behaviours. Furthermore, because G. pennsylvanicus is found in high densities in both urban and rural environments across North America, including Ottawa, information contained in the acoustic social environment has the potential to be impeded by anthropogenic noise. I therefore also investigated whether the presence of anthropogenic noise interrupted or corrupted the information contained in the acoustic social environment, thereby altering how juvenile development and adult mating behaviours were affected. 


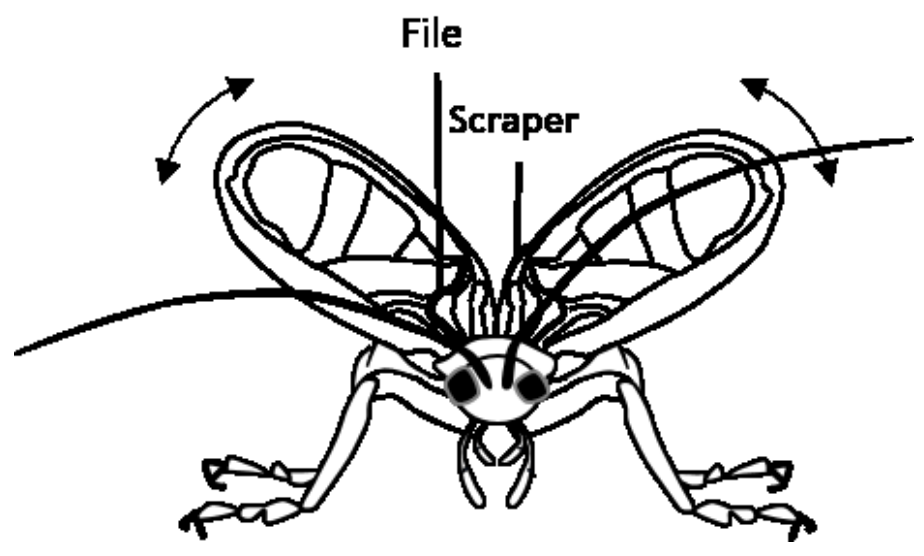

Figure 1.1. A male field cricket with its forewings raised in signal production. The file on the right wing is brushed across the scraper on the left wing during the closing stroke of the wings, producing a single pulse of sound. Pulses are concatenated into chirps (e.g., Figure 1.2). 


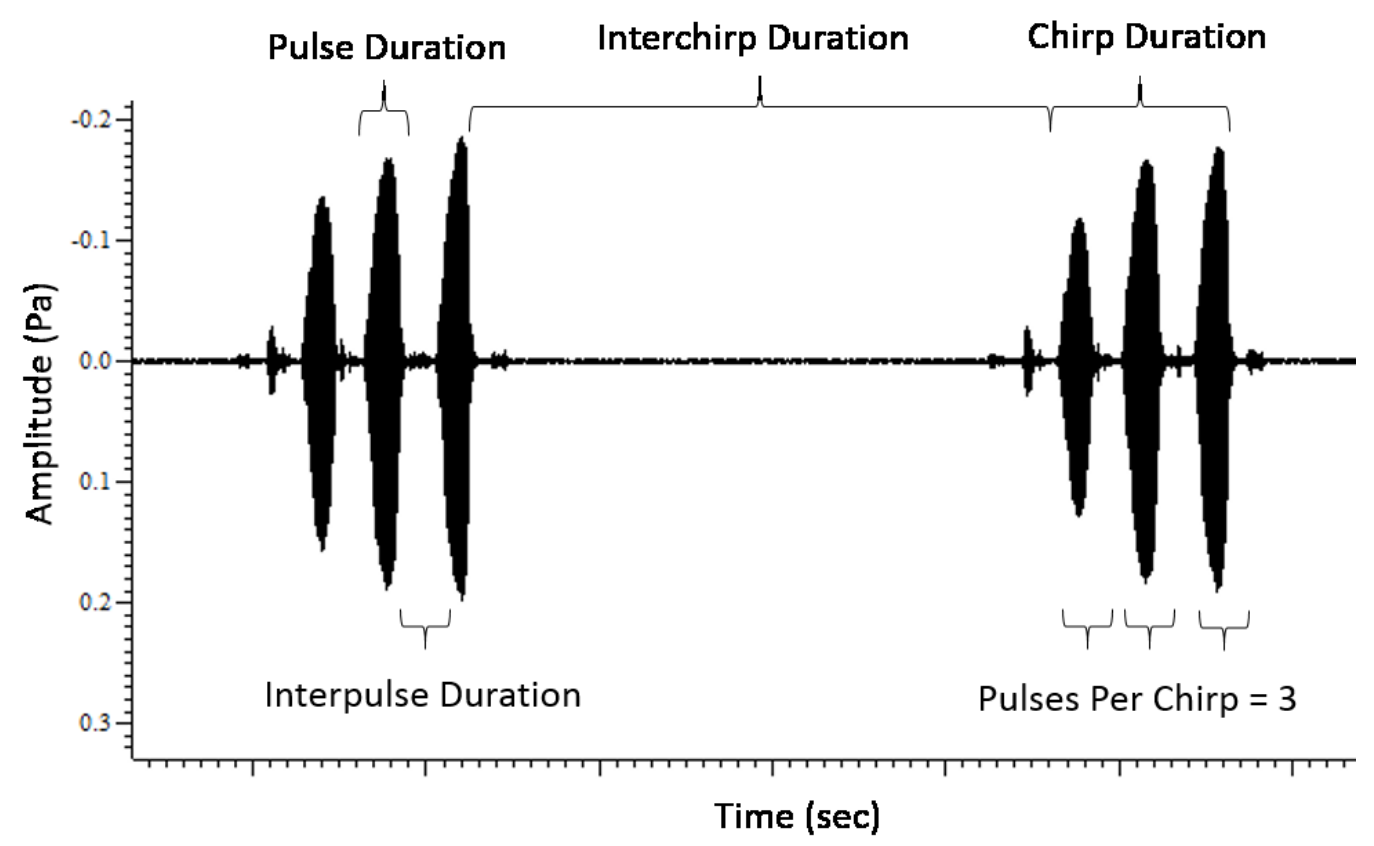

Figure 1.2. Waveform of 2 chirps from a G. pennsylvanicus long distance mate attraction signal displaying some of the various signal parameters I quantified. 


\subsection{Thesis Rationale and Objectives}

For my Doctoral thesis research, I exposed juvenile fall field crickets, (Gryllus pennsylvanicus), to different acoustic environments to investigate how the acoustic environment experienced during development affected life history traits and adult behaviour. Because the overall plasticity of an individual is the result of a concomitant interaction between development, environment, and behaviour over the entire lifetime, my empirically based research dissertation focused on the integrative phenotype created by the interaction of different life stages. Specifically, I investigated how behavioural plasticity was constrained by concomitant variation in developmental plasticity so that I could create a more complete understanding of how plasticity creates dynamic phenotypes for selection to act upon. To do this, I exposed juvenile fall field crickets to one of three acoustic environments that differed in the social density of adult males.

To build my different acoustic social environments I manipulated the number of acoustic mate attraction signals being broadcast to represent a high density acoustic social environment (High), a low density acoustic social environment (Low) and an environment without any acoustic mate attraction signals (None representing an acoustic social isolation environment). Further, because the fall field cricket occurs in both rural and urban environments, I simultaneously examined the impact of anthropogenic noise on developmental and behavioural plasticity. To do so, I overlaid these acoustic social environments with anthropogenic noise, resulting in a total of six different acoustic environments. I then exposed individual field crickets to their acoustic environment from 1 week post hatch through adulthood. Chapter 2 provides a detailed description of the overall methodology I used to quantify how fall field crickets responded to these different 
acoustic environments.

Using the methodological approach I outline in Chapter 2, I then quantified how fall field crickets responded plastically to different acoustic environments. Specifically, in Chapter 3 I investigated how the acoustic social environment and anthropogenic noise experienced during development influenced fall field cricket life-history traits such as development time, body size and residual mass at adulthood in both males and females. In the remaining chapters my focus shifted to investigating how the acoustic environment during development influenced adult behavioural plasticity, Chapters 4 and 5 focused on adult male behaviour, Chapter 6 focused on adult female behaviour, while Chapter 7 focused on how adult male behaviour is affected by a change in environments between development and adult eclosion. Male field crickets fight for access to signalling territories from which they produce acoustic mate attraction signals to attract potential mates. Therefore, in Chapter 4 I examined how the acoustic social environment and anthropogenic noise experienced during development influenced male aggressive behaviour in adulthood. In Chapter 5 I examined how the acoustic social environment and anthropogenic noise experienced during development influenced adult male mate attraction signalling behaviour. Examining aggression and signalling behaviour captures the two main components of adult behaviour that most strongly impact male fitness. In Chapter 6, I switched my attention to females. Female field crickets are attracted to males by the male acoustic mate attraction displays, often preferentially mating with males who produce the most energetically demanding mate attraction signals. I therefore examined how the acoustic social environment and anthropogenic noise experienced during development influenced adult female mating preferences. 
In Chapters 4-6 I examined behavioural plasticity after the adults had been continuously exposed to their assigned acoustic environment throughout their juvenile development and into adulthood. However, to create a more complete understanding of how plasticity creates dynamic phenotypes for selection to act upon, it was essential that I examine how behavioural plasticity was constrained by concomitant variation in developmental plasticity. To do this, I had to ascertain how the developmental and adult acoustic environments interact to influence adult behaviour. To investigate interactions, individuals must experience different environments between juvenile and adult stages. Therefore, in Chapter 7 I examined how the acoustic social environment during development interacts with the acoustic social environment during adulthood. Specifically, I reared males in either the High or None acoustic social environments and then switched environments at adult eclosion for half of the males, while maintaining the same environments for the remaining males. I then analyzed their acoustic mate attraction signalling behaviour. In this manner, I was able to determine whether adult males could dynamically adjust to changes in their social environment using behavioral plasticity, or if their adult behaviour was too constrained from their potentially miss-matched developmental environment.

Together my dissertation research allowed me to determine whether juvenile fall field crickets (Gryllus pennsylvanicus) that eavesdropped on the acoustic mate attraction signals of adult conspecifics (presented at different densities) plastically altered their life history trajectories during development. My dissertation simultaneously investigated whether adults exhibited behavioural plasticity in response to the social density information that was contained in the signals that they overheard. Concurrently, I 
investigated whether the addition of anthropogenic noise during development influenced the juvenile field crickets' ability to properly access the acoustic social environment and whether this plastically altered life history traits or adult behaviours. Because my research examined plasticity throughout the entire life-history of the fall field crickets, I was able to capture the impact of how the acoustic environment experienced during development and adulthood influenced developmental plasticity, behavioural plasticity, and their interactions. 


\section{Chapter 2: General Methods}

\subsection{Model Study Species}

I conducted my study in accordance with the guidelines of the Canadian Council on Animal Care. I used the North American fall field cricket (Gryllus pennsylvanicus) as my model study organism. My research was not reviewed by an animal care committee as the Canadian Council on Animal Care does not require research on insects to be reviewed. While no field-collecting or import permits were required to collect or house $G$. pennsylvanicus, the facility we used to house this population had Plant Pest Containment Level 1 rating (PPC1 permit \# PC-2014-053).

The fall field crickets I used in my experiments were from an established lab population of G. pennsylvanicus that was initially collected in August 2010 from Koffler Scientific Reserve (University of Toronto) at Jokers Hill in the Oak Ridges Moraine in King Township, ON. Genetic variation in this lab population was maintained with the annual introduction of new wild stock collected from the Carleton University Campus, Ottawa ON.

\subsection{General Experimental Protocol}

I provided colony G. pennsylvanicus adults with damp sand $(250 \mathrm{ml})$ and allowed them to deposit eggs for 2 weeks. Sand cups were then placed in a communal rearing colony bin (L x W $\times \mathrm{H}=64 \times 40 \times 42 \mathrm{~cm}$ ). All rearing adults and hatchlings were housed in a greenhouse at Carleton University, under a 14h:10h light:dark photoperiod at $28 \pm 2{ }^{\circ} \mathrm{C}$. Thrice weekly (on a Monday, Wednesday, Friday schedule) 25 - 30 newly hatched nymphs were removed and placed into a separate clear plastic container ( $\mathrm{L} \times \mathrm{W} \times \mathrm{H}=$ 
$16.5 \times 10 \times 16.5 \mathrm{~cm}$ ) with a vented lid and provided with fresh water, ad libitum food (Harlan Teklad Inc. Rodent diet no. 8604M), and cardboard (egg carton) shelters. One week after hatching I placed each of the nymphs into their own individual clear plastic containers $(\mathrm{L} \times \mathrm{W} \times \mathrm{H}=10 \times 7 \times 7 \mathrm{~cm}$ ) and provided them with the same food, water, and shelter regime. Lids were aerated to allow light, airflow, and sound.

I randomly assigned each one-week old cricket to one of 6 acoustic environment treatments (see below) and placed them (in their individual housing) into a larger communal treatment bin $(\mathrm{L} \times \mathrm{W} \times \mathrm{H}=79 \times 39 \times 46 \mathrm{~cm})$. Since the general structure of cricket tympanic organs are present at hatching and precocious hearing may be possible, using one-week old crickets ensured that juveniles would not experience any physical social interactions and that their acoustic environment would be set as their hearing fully developed around the sixth instar (Ball \& Young 1974). The communal treatment bins could accommodate 20 individual cricket houses simultaneously, split between 2 levels. The communal treatment bins and the lid were lined with acoustic foam with an NRC rating of 0.80 to prevent sound contamination across treatments. A light source (IKEA Dioder LED light, warm white-2700 Kelvin) was inset into the lid and the crickets were kept on the 14h:10h light:dark photoperiod at $28 \pm 2{ }^{\circ} \mathrm{C}$. Each communal treatment bin lid also contained 4 speakers (StarBurst 3.5mm from Vibe Sound), pointing down at the containers ( $25 \mathrm{~cm}$ from the nearest individually housed cricket) for playback of acoustic environment soundtracks. The individual cricket containers were rotated around the communal treatment bin on a thrice weekly schedule to ensure that light and sound exposure was consistent for every individual throughout the experiment. Thrice weekly, each juvenile cricket also had its food and water replenished and was checked for wing 
pad eclosion [approximately $3^{\text {rd }}-2^{\text {nd }}$ instar from imaginal (adult) moult], and adult eclosion. Once crickets reached adult eclosion they were removed and weighed using a Pioneer Balance (model PA214, OHAUS Corporation, USA). Adult crickets were either euthanized via freezing (Chapter 3) or placed into adult experimental bins for behavioural trials (Chapters 4 - 7). New individual nymphs were added to the experiment weekly to replace eclosed adults and any that died before adulthood. All adults eclosed between February 2015 and August 2017.

\subsection{Acoustic Environment Soundtracks}

To test the effects of how the acoustic environment influenced juvenile development and adult mating behaviours, I created 6 different acoustic soundtrack treatments using a $3 \times 2$ factorial design: 3 with different densities of male acoustic signals ( 3 different acoustic social environments), and 3 with these male acoustic signals overlaid with anthropogenic noise (Table 2.1, explained below). Each acoustic soundtrack was broadcasted into one of the large communal treatment bins, resulting in 6 different acoustic environment soundtrack treatments.

To create the male acoustic signal soundtracks, I recorded the long distance mate attraction signals of 32 randomly selected adult male G. pennsylvanicus from the lab colony over a 4-day period using the Electronic Acoustic Recording System (EARS-II; designed and developed by Cambridge Electronic Design, Cambridge, UK). I isolated the acoustic signals from 3 males with the highest time spent signalling to ensure adequate waveform files. Using Adobe Audition CS6 (version 5.0), I sampled 2, 30-second sound bites from each of the 3 males to create individual signals, each 2.7 minutes in length 
(mean signal duration from the original 32 males). Inter-signal duration was calculated using mean daily time spent signalling (from the 32 males) and 4-hour signalling bouts were used to create a 24-hour soundtrack for each of the 3 males. Signalling and silence durations over the 24 hours were altered to emulate the natural daily signalling pattern of G. pennsylvanicus (Souroukis et al. 1992; Alexander \& Meral 1967; French \& Cade 1987) (Figure 2.1). The 3 males' soundtracks were then overlaid together, with varied start times between the males so that the chirps did not overlap during playback (Figure 2.1). By altering the number of these 24-hour soundtracks mixed together, I manipulated the density of adult males in each of the soundtracks.

My protocol resulted in 3 different acoustic social environment soundtracks, each created to represent different levels of perceived future adult male population densities. The Low density (Low) soundtrack consisted of the 3 individual males (Figure 2.1). The High density (High) soundtrack consisted of the same 3 males repeated 4 times, representing 12 separate male signals (4 times the density; Figure 2.1). I repeated the 3 original males to ensure that the differences between the low and high acoustic social environments represented variation in the number/density of signalling males and not variation in the male quality of signal parameters. Thus, juvenile crickets in the Low and High density treatments experienced acoustic mate attraction signalling from the same three males during development, with varying repetition, but not variation in male quality. The third environment was a social acoustic isolation environment (None), consisting of empty room noise playback (downloaded from www.freesound.org).

To test the effects of an urban environment, anthropogenic noise was then overlaid onto these crickets' signals. To ensure that the noise was representative of what 
wild crickets would encounter, the amplitude and type of anthropogenic noise on the Carleton University campus (a collection site for the crickets that make up the lab population) was measured over several 48 -hour periods using a digital sound level meter (Extech Instruments, Model 407750). High quality anthropogenic sound clips were obtained using free sound files from SoundBible (http://soundbible.com). Multiple noise types (traffic, construction, sirens, foot traffic) were then compiled into a 24-hour soundtrack using Adobe Audition CS6 (version 5.0) to represent typical, daily anthropogenic noise that temporally varied over the course of the day and night. When overlaid onto the 3 different acoustic social environment soundtracks, a total of 6 different acoustic environments were represented (Table 2.1). Tracks were played continuously on a 24-hour loop ( 7 days a week) through the 4 speakers in the container lid using USB powered Mp3 players (Hipstreet HS-529 4GB) and at the average amplitude of $56.24 \mathrm{db}$ measured in the middle of the container (30 $\mathrm{cm}$ from the speakers) using a EXTECH Digital Sound Level Meter (Model \# 407768). 


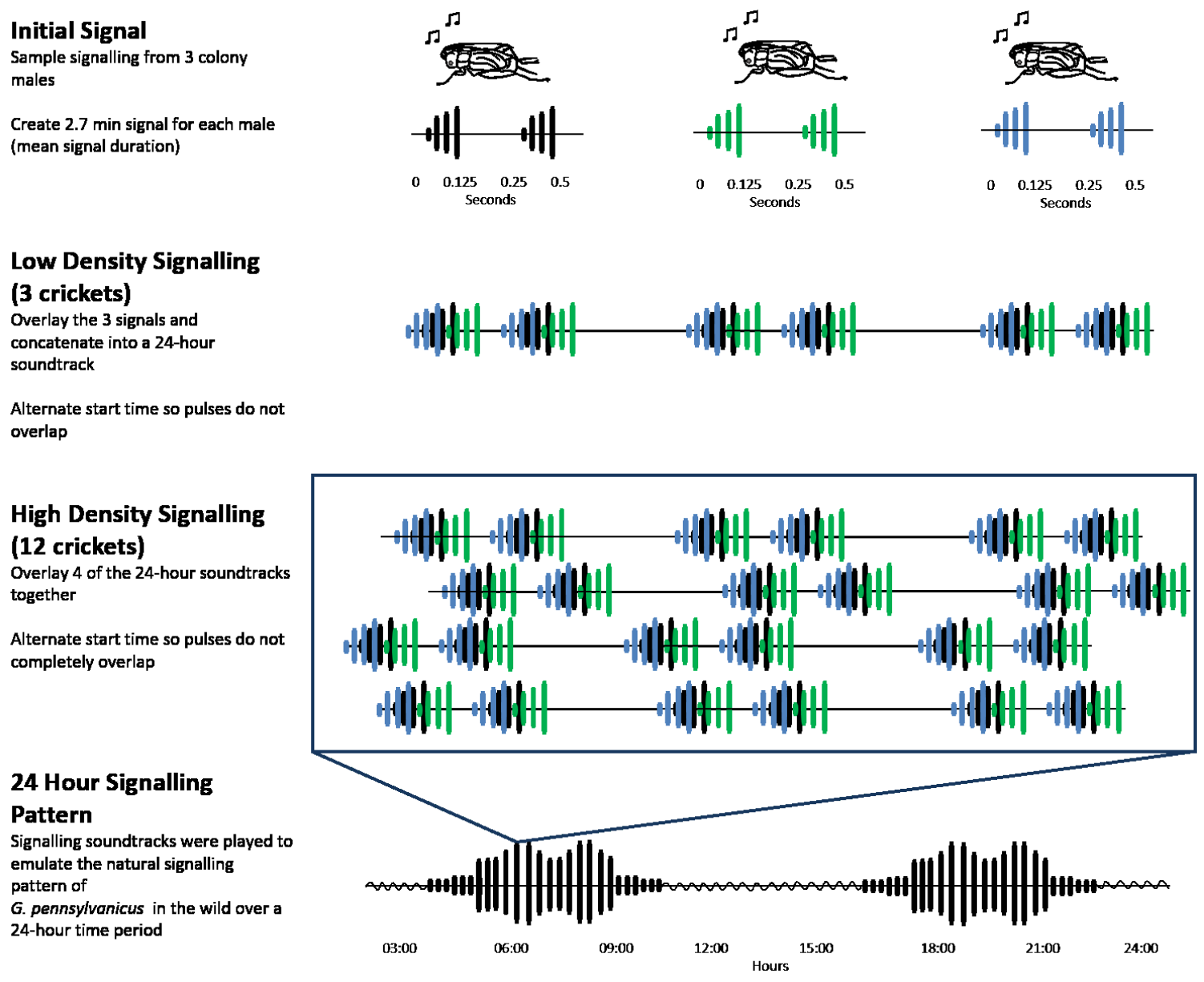

Figure 2.1. Stylized schematic representing how the environmental soundtracks were created. Shown above are samples from the Low density Soundtrack, consisting of signals from 3 separate males; and the High density Soundtrack, which consisted of the Low Soundtrack overlaid 4 times resulting in a total of 12 male signals. These signals were played across a 24-hour period that mimicked the natural temporal shifts in signalling patterns of $G$. pennsylvanicus. 
Table 2.1. Acoustic environment treatments. Juveniles were raised in one of six acoustic treatments that represent differing levels of adult male calling (signalling density) and anthropogenic noise.

\section{Signalling Density Treatment}

High Low None

\begin{tabular}{cccc}
\hline Anthropogenic Noise & & & \\
Yes & High/Yes & Low/Yes & None/Yes \\
No & High/No & Low/No & None/No \\
\hline
\end{tabular}




\subsection{Photographs and Measurements}

Once euthanized, each adult cricket was photographed from above in the dorsal view using a digital camera (Panasonic LUMIX DMC-ZS40 30X) with a visible scale. The photos were used to obtain 3 standard body size measurements: pronotum width (maximal distance transversely across the pronotum), pronotum length (maximal distance down the sagittal length of the pronotum), and maximum head width (maximal distance between the outer edges of the eyes) (Figure 2.2). Measurements were taken using ImageJ (version 1.48, National Institute of Health). To ensure reliability of the measurements, photographing and measuring abilities were tested (99\% concordance). 


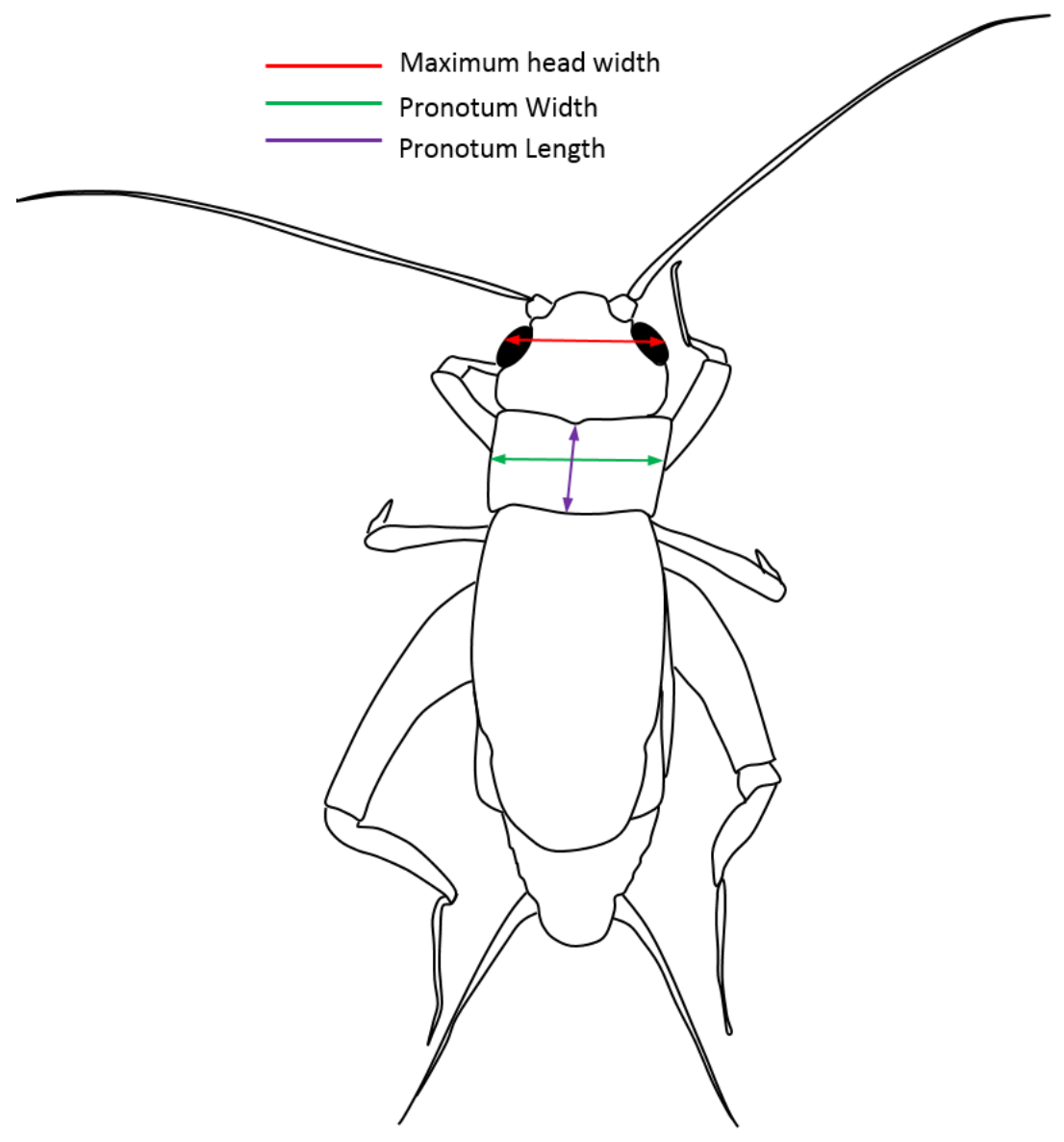

Figure 2.2. Schematic depicting the three standardized measurements used to determine adult body size in both male (pictured) and female crickets. 


\section{Chapter 3: Developmental acoustic social environment and}

\section{anthropogenic noise affects field cricket expression of life history traits}

\subsection{Abstract}

An individual's ability to change their phenotypic expression throughout development and adulthood to suit the changing environmental conditions they experience has the potential to affect their fitness. Historically researchers have focused on phenotypic response to changes in the physical environment. However, changes in the social environment may also be important. Juveniles may allocate developmental resources based on the social environment they expect to face at maturation and adults may adapt their behaviour to match changing social conditions. Understanding how animals react to changes in social environment across different life stages (developmental versus adulthood) is therefore central to fully comprehend the factors driving phenotypic plasticity. Eavesdropping on the acoustic signals of conspecifics (the acoustic social environment) allows individuals to continuously monitor their social environment. However, an individual's ability to hear signals may be interrupted or altered by anthropogenic noise. I therefore quantified the extent of developmental plasticity in response to adult mate attraction signalling (the acoustic social environment) and urban noise. I reared male and female fall field crickets, Gryllus pennsylvanicus, while exposing them to one of six different acoustic environments that differed in the density of signalling adult males (High, Low and None) and anthropogenic noise (Present or Absent). Exposure to adult male signals during development resulted in decreased development time in both females and males. When exposed to anthropogenic noise, males in the Low density environment also developed faster. Neither male adult size nor 
residual mass was affected by the acoustic environment they were exposed to during rearing; however, females exposed to higher signalling densities were larger at adult eclosion. Females raised exposed to anthropogenic noise without acoustic mate attraction signals had smaller residual mass at eclosion compared to females raised without the noise playback. I also found indirect effects, as adult residual mass (both sexes) and body size (males only) were both affected by development time. Females that developed faster had lower residual mass, while males that developed faster were both larger and had lower residual mass. Together, the direct and indirect effects of the social environment juveniles overhear while developing support theoretical predictions of developmental plasticity, as juveniles altered their life history traits in response to differences in expectant adult population levels. My results also provide evidence that anthropogenic noise pollution early in life may interfere with an individual's ability to accurately assess their future social environment, leading to phenotypic and environmental mis-matching.

\subsection{Introduction}

Previous work on phenotypic plasticity, both developmental and behavioural, has primarily focused on plastic responses to large-scale environmental fluctuations. However, a handful of recent studies have shifted towards evaluating phenotypic plasticity in response to changes within the social environment. While this idea has been relatively unexplored (Kasumovic \& Brooks 2011), research indicates that demographic changes in both the surrounding adult and concurrent juvenile populations can provide cues about contemporary and future competition, mate availability, and general population densities (Kasumovic \& Brooks 2011). Developmental plasticity seems to be 
affected by the social environment, as juveniles can and will allocate resources differently depending on the expectant social environment they will face as adults (Kasumovic et al. 2011; Kasumovic, et al. 2012 b). For example, social information during development affects the rate of development in the redback spider (Latrodectus hasselti) (Kasumovic \& Andrade 2006), the size of adults as well as accessory glands in male fruit flies (Drosophila melanogaster) (Bretman et al. 2016), testis size in yellow dung flies (Scatophaga stercoraria) (Stockley \& Seal 2001), adult activity levels in zebra fish (Danio rerio) (Shams et al. 2017), and adult sexual behaviour in black field crickets (Teleogryllus commodus) and mustard leaf beetles (Phaedon cochleariae) (Kasumovic et al. 2011; Kasumovic, et al. 2012 b; Tremmel \& Muller 2013).

Animals can use a variety of methods to determine the make-up of their social environment, including tactile cues (Stockley \& Seal 2001), vibratory cues (Cocroft et al. 2005), chemical cues (Hedrick \& Kortet 2012), visual cues (Milner et al. 2008), and acoustic cues (Kasumovic et al. 2011; Kasumovic, et al. 2012 b). Of these, acoustic cues provides a unique situation for researchers because, unlike chemical or visual signals, acoustic signals are relatively easy to observe and record (Otte 1974). However, as acoustic systems exist as part of an active living environment, any acoustic signal - and thus the information it conveys - has the potential to be overheard by listeners other than the intended receivers. These 'eavesdroppers' can use information present in the signal to help them assess the make-up of their social environment.

Information contained in the acoustic signals also has the potential to be interrupted or corrupted by noise and noise pollution. Communication can be affected by elevated noise levels, altered sound channels, amplitude shifts, and interfering 
frequencies (Warren et al. 2006; Pijanowski et al. 2011). This may include noise emanating from roads, hydro corridors, airports, and urban centres (Warren et al. 2006). If interference is great enough, it is possible that anthropogenic noise disturbance could lead to the acoustic divergence of urban and nonurban populations (Smith et al. 2008). Thus, as urbanization creates novel physical and acoustic landscapes, animals are forced to either adapt or abandon their habitats.

Depending on the source, anthropogenic noise can have a sound pressure level (SPL) of $20 \mathrm{~dB}$ (whisper) to over $100 \mathrm{~dB}$ (airplanes)(Rabin et al. 2003; Theebe 2004). The majority of noise created by human environments is concentrated at amplitudes between 40-70 dB (Theebe 2004; I. Bateman et al. 2001). Urban noise pollution has been shown to disrupt communication in several bird and frog species, including song sparrows (Melospiza melodia) (Wood \& Yezerinac 2006), great tits (Parus major) (Mockford \& Marshall 2009), and tree frogs (Hyla arborea) (Lengagne 2008). Because of the noisy acoustic environments, animals may either alter the frequency or the amplitude of their signals in order to increase the audibility of their communication (Katti \& Warren 2004). Noise management of both rural and urban human habitats has become a major issue in environmental protection and urban planning (Kight \& Swaddle 2011; Laiolo 2010). However, research examining how anthropogenic noise effects development, especially longitudinal data, is sparse. Thus, a more thorough understanding of how anthropogenic noise during development effects life history trait plasticity is necessary to appreciate the full evolutionary consequences of noise pollution (Barber et al. 2010; Kight \& Swaddle 2011). Given this, here I examine how developmental life history traits of the North American fall field cricket are affected by 
exposure to both acoustic social information, indicative of different adult male cricket densities, and anthropogenic noise pollution.

\subsection{Methods}

\subsubsection{Study Animals}

My study species is the North American fall field cricket, Gryllus pennsylvanicus. Gryllus pennsylvanicus in an ideal species for my study because it is found in high densities in both urban and rural environments across North American, including Ottawa, Canada, suggesting it can handle both minimal (rural) and extreme (urban) levels of anthropogenic noise. Field crickets are also ideal for my study because they express a complex mating system that is highly reliant on acoustic communication in the form of male long-distance mate attraction signals and courtship songs (Alexander 1961). These acoustic sexual signals have the potential to convey important information about the male composition of the current social cohort, including density and male fitness (Otte 1974). Thus, sexual signals can provide acoustic information about the social environment that have the potential to influence several aspects of juvenile development and adult behaviour such as development time, body size at adulthood, female mate choice (Souroukis \& Cade 1993; Souroukis \& Murray 1994), male aggression and acoustic mate attraction signalling (Alexander 1961).

\subsubsection{General Experimental Protocol}

To test how development in field crickets is affected by their acoustic social environment, I randomly assigned one-week old crickets to one of 6 acoustic environment treatments and placed them into a larger communal treatment bin that was lined with acoustic foam. 
Each communal treatment bin lid contained 4 speakers for playback of the treatment's acoustic environment soundtracks. I used 6 different acoustic social environment soundtrack treatments in a $3 \times 2$ factorial design: 3 with different densities of male acoustic mate attraction signals (High, Low, None), and 3 with these acoustic signals overlaid with anthropogenic noise (Table 2.1; Figure 2.1). Thrice weekly, each juvenile cricket was checked for wing pad eclosion or adult eclosion. I exposed both males and females to their acoustic social environment treatments, and then quantified development rate, body size, and residual mass at adulthood for all individuals. This allowed me to determine whether males and females use the social information present in acoustic signals (different densities of expected adult cohorts) to plastically alter the expression of their developmental life history traits. Further, to ascertain if anthropogenic noise might influence the plasticity of cricket developmental trajectories, I examined how the addition of anthropogenic noise to the acoustic play-backs affected cricket developmental traits. Chapter 2 provides a detailed description of how I produced my six different acoustic treatments, how I housed my individual crickets during rearing in their different acoustic treatments, how I quantified body size, and the other general protocols that I used for all data chapters.

\subsubsection{Statistical Analyses}

All statistical analyses were completed using JMP statistical software (version 11.0.0 SAS Institute). To quantify adult body size, I combined body size measurements using a principle component analysis (PCA) by sex to extract orthogonal vectors from pronotum length, pronotum width, and head width. The first principal component ( $\mathrm{PC1}$ size) explained $87 \%$ of the variation in female size (Eigenvalue $=2.61$ ) and $92 \%$ of the 
variation in male size (Eigenvalue $=2.77)$; all size measures loaded equally and positively on PC1 size (eigenvectors ranged from 0.56 to 0.59 for both sexes). To quantify residual mass, I regressed adult Body size (PC1 size) against body weight quantified at adult eclosion.

I used linear models (LMs) to examine the effects of the acoustic environment experienced during rearing on cricket development time, adult body size (PC1 size), and adult residual mass. I quantified two measures of development time: time in days from Hatching to Wing Pad eclosion (at which point sex could be determined), and time in days from Wing Pad eclosion to Adult eclosion. Total development time (Hatching to Adult Eclosion) was calculated by summing these two values. For my LMs the dependent variables were the following life history traits: days from Hatching to Wing pad eclosion, days from Wing pad eclosion to Adult eclosion, days from Hatching to Adult eclosion (total development time), adult body size (PC1 Size), and adult residual mass. I included the following independent variables in each LM: signalling density (High, Low, None), anthropogenic noise (Yes, No), and their interactions. I ran a separate LM to explore how development time on its own affected body size (PC1 size) and residual mass at adulthood. Here the independent variables were days from Hatching to Wing pad eclosion and days from Wing pad eclosion to Adult eclosion. Because I ran my experiment over a 12 month period, I also included season of hatching (Winter, Spring, Summer, Fall) as a covariate to control for possible block effects in all LMs.

I tested all LMs for violations of normality by examining the distribution of residual diagnostic plots (residuals vs fitted values and Q-Q plots); I also tested all LMs for violations of homoscedasticity by looking for dependency between the residuals and 
the fitted values. Normality and homoscedasticity assumptions were not violated. Pvalues were corrected using false discovery rate FDR $_{\mathrm{B}-\mathrm{Y}}$ method (Benjamini \& Yekutieli 2001). For models with significant effects, I examined how the dependent variable changed across treatments using pairwise Tukey's HSD contrasts or student's t-tests (Post Hoc comparisons).

\subsection{Results}

Of the 882 nymphs I placed into the experiment, 513 successfully reached adulthood: 285 females (High density: Anthropogenic noise 50, No Anthropogenic noise 51; Low density: Anthropogenic noise 49, No Anthropogenic noise 54; Isolation: Anthropogenic Noise 40, No Anthropogenic noise 41) and 228 males (High density: Anthropogenic noise 40, No Anthropogenic noise 40; Low density: Anthropogenic noise 43, No Anthropogenic noise 36; Isolation: Anthropogenic Noise 37, No Anthropogenic noise 32). There was no difference in the distribution across treatments of the 272 nymphs that died during development $\left(\chi^{2}=0.85, \mathrm{df}=5, P=0.97\right)$. Significantly more females than males reached adulthood than was expected by chance alone (Exact binomial test of goodness-of-fit, $P=0.013)$. It is impossible to determine whether the greater proportion of females surviving resulted from the oviposition of more female than male eggs, or from males dying at a slightly higher rate than females during development, as most crickets died within the first 3.5 weeks, before their sex could be identified (days till death of crickets that died during development: $\bar{x} \pm S E=25.4 \pm 1.24)$. Females developed 5 days faster than males (hatching to adulthood: females $\bar{x} \pm S E=48.7 \pm 0.3$; males $\bar{x} \pm S E=50.5 \pm 0.4 ;$ ANOVA: $\mathrm{R}^{2}$ adj $\left.=0.02, \mathrm{~F}_{1,513}=13.5, P=0.0003\right)$ and 
weighed almost $100 \mathrm{mg}$ more at adulthood (females $\bar{x} \pm S E=435.3 \pm 5.8 \mathrm{mg}$; males $\bar{x} \pm S E=338.1 \pm 6.5 ;$ ANOVA: $\left.\mathrm{R}^{2}{ }_{\text {adj }}=0.19, \mathrm{~F}_{1,513}=13.5, P=0.0001\right)$. Given these sex differences, below I separated the sexes to explore how social environment and anthropogenic noise impact life history traits in females and males.

\subsubsection{Impact of Acoustic Treatment on Female Developmental Traits}

Female development time was significantly affected by the acoustic environmental treatment (Table 3.1). Females raised in the High and Low density acoustic social environments developed significantly faster than females raised in the None (acoustic isolation) treatment $(P<0.0001$; Table 3.1; Figure 3.1). Specifically, females in the High and Low density acoustic environment treatments developed wing pads 3 days sooner $($ High $\bar{x} \pm S E=31.6 \pm 0.45 ;$ Low $\bar{x} \pm S E=31.8 \pm 0.45 ;$ None $\bar{x} \pm S E=34.4 \pm 0.50$ days); and reached adult eclosion 4 days sooner (High $\bar{x} \pm S E=47.2 \pm 0.49$; Low $\bar{x} \pm$ $S E=47.7 \pm 0.49 ;$ None $\bar{x} \pm S E=51.9 \pm 0.55$ days) than females raised in the acoustic isolation (None) treatment.

Acoustic treatment also significantly influenced female body size at adulthood $(P$ $=0.0197$; Table 3.1; Figure 3.2). Specifically, females raised in the High density acoustic social environment were significantly larger at adult eclosion than females raised in the Low density acoustic social environment. Neither development time nor body size at adulthood in females was affected by the addition of anthropogenic noise.

Female residual mass at adult eclosion was influenced by a significant interaction between Signalling density x Anthropogenic Noise $(P=0.0053$; Table 3.1; Figure 3.3). In the absence of anthropogenic noise, female crickets raised in acoustic isolation (None treatment) were significantly heavier at adult eclosion (after controlling for body size) 
than those raised in the High density treatment. Conversely, when anthropogenic noise was added, females reared in acoustic isolation (None treatment) were significantly lighter at adult eclosion compared to females reared in the High density treatment.

Females raised in isolation with anthropogenic noise were also significantly lighter at adult eclosion than females raised in isolation raised without anthropogenic noise (Figure $3.3)$. 
Table 3.1. Results of the linear models examining effects of acoustic treatment on development time measures, adult body size (PC1 size) and adult residual mass in females. Significant effects are bolded for both models and model effects, FDR $\mathrm{B}_{\mathrm{B}} \mathrm{Y}$ corrected $P=0.02$ $(\mathrm{N}=285)$. Parameter estimates are included in Appendix 1, Table A1.1.

\begin{tabular}{|c|c|c|c|c|c|c|c|}
\hline Model & $\mathbf{R}^{2}$ adj & $\mathbf{F}_{\text {model }}$ & $\mathbf{P}_{\text {model }}$ & Model Effect & DF & $\mathbf{F}$ & $\mathbf{P}$ \\
\hline \multirow[t]{4}{*}{ Hatching to Wing pad } & 0.12 & 5.90 & $<0.0001$ & Season & 3 & 7.58 & $<0.0001$ \\
\hline & & & & Signalling Density & 2 & 12.02 & $<0.0001$ \\
\hline & & & & Anthropogenic Noise & 1 & 0.38 & 0.54 \\
\hline & & & & Signalling Density*Anthropogenic Noise & 2 & 1.56 & 0.21 \\
\hline \multirow[t]{4}{*}{ Wing pad to Adult eclosion } & 0.18 & 8.76 & $<0.0001$ & Season & 3 & 6.41 & 0.0003 \\
\hline & & & & Signalling Density & 2 & 25.15 & $<0.0001$ \\
\hline & & & & Anthropogenic Noise & 1 & 0.04 & 0.83 \\
\hline & & & & Signalling Density*Anthropogenic Noise & 2 & 1.44 & 0.24 \\
\hline \multirow[t]{4}{*}{ Hatching to Adult eclosion } & 0.21 & 10.50 & $<0.0001$ & Season & 3 & 10.17 & $<0.0001$ \\
\hline & & & & Signalling Density & 1 & 27.43 & $<0.0001$ \\
\hline & & & & Anthropogenic Noise & 1 & 0.23 & 0.63 \\
\hline & & & & Signalling Density*Anthropogenic Noise & 2 & 1.86 & 0.16 \\
\hline \multirow[t]{4}{*}{ PC1 size } & 0.03 & 2.00 & 0.05 & Season & 3 & 1.87 & 0.14 \\
\hline & & & & Signalling Density & 2 & 3.99 & 0.0197 \\
\hline & & & & Anthropogenic Noise & 1 & 0.26 & 0.61 \\
\hline & & & & Signalling Density*Anthropogenic Noise & 2 & 0.88 & 0.41 \\
\hline \multirow[t]{4}{*}{ Residual Mass } & 0.01 & 1.54 & 0.15 & Season & 3 & 0.46 & 0.71 \\
\hline & & & & Signalling Density & 2 & 0.03 & 0.97 \\
\hline & & & & Anthropogenic Noise & 1 & 0.08 & 0.78 \\
\hline & & & & Signalling Density*Anthropogenic Noise & 2 & 5.35 & 0.0053 \\
\hline
\end{tabular}




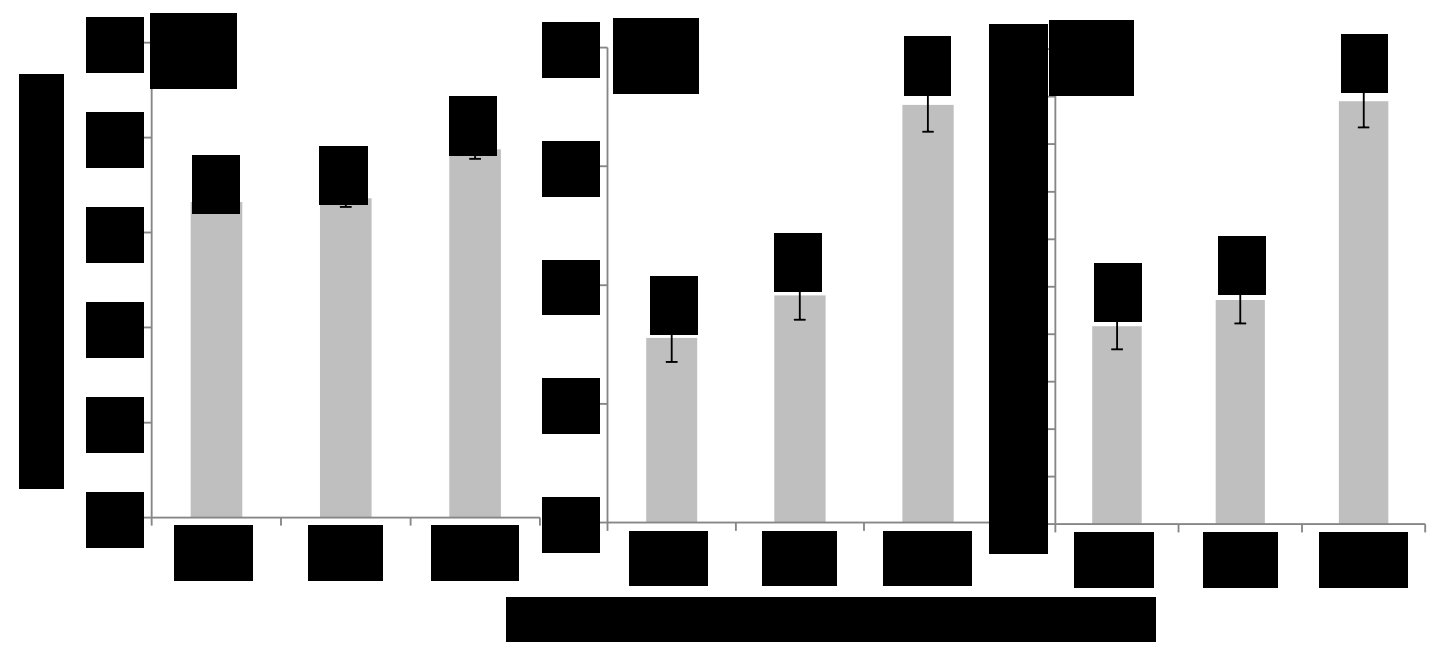

Figure 3.1. How the acoustic social environment during development influences female development time across the different life stages: (A) Hatching to Wing pad, (B) Wing pad to adult eclosion, and (C) Total Development Time. Error bars represent standard errors and lowercase letters denote significant differences between acoustic treatment groups for each life stage. 


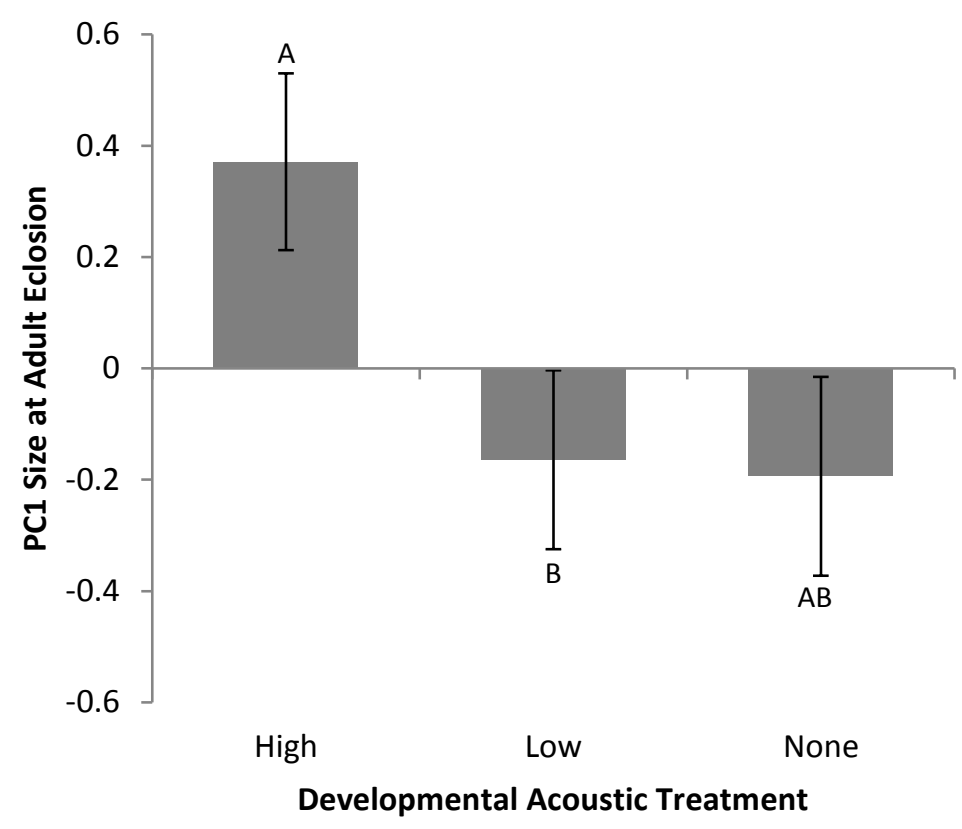

Figure 3.2. How the acoustic social environment during development influences female body size (PC1 size) at adult eclosion. Bars represent the average PC1 size and error bars are standard errors; letters denote significant differences. 


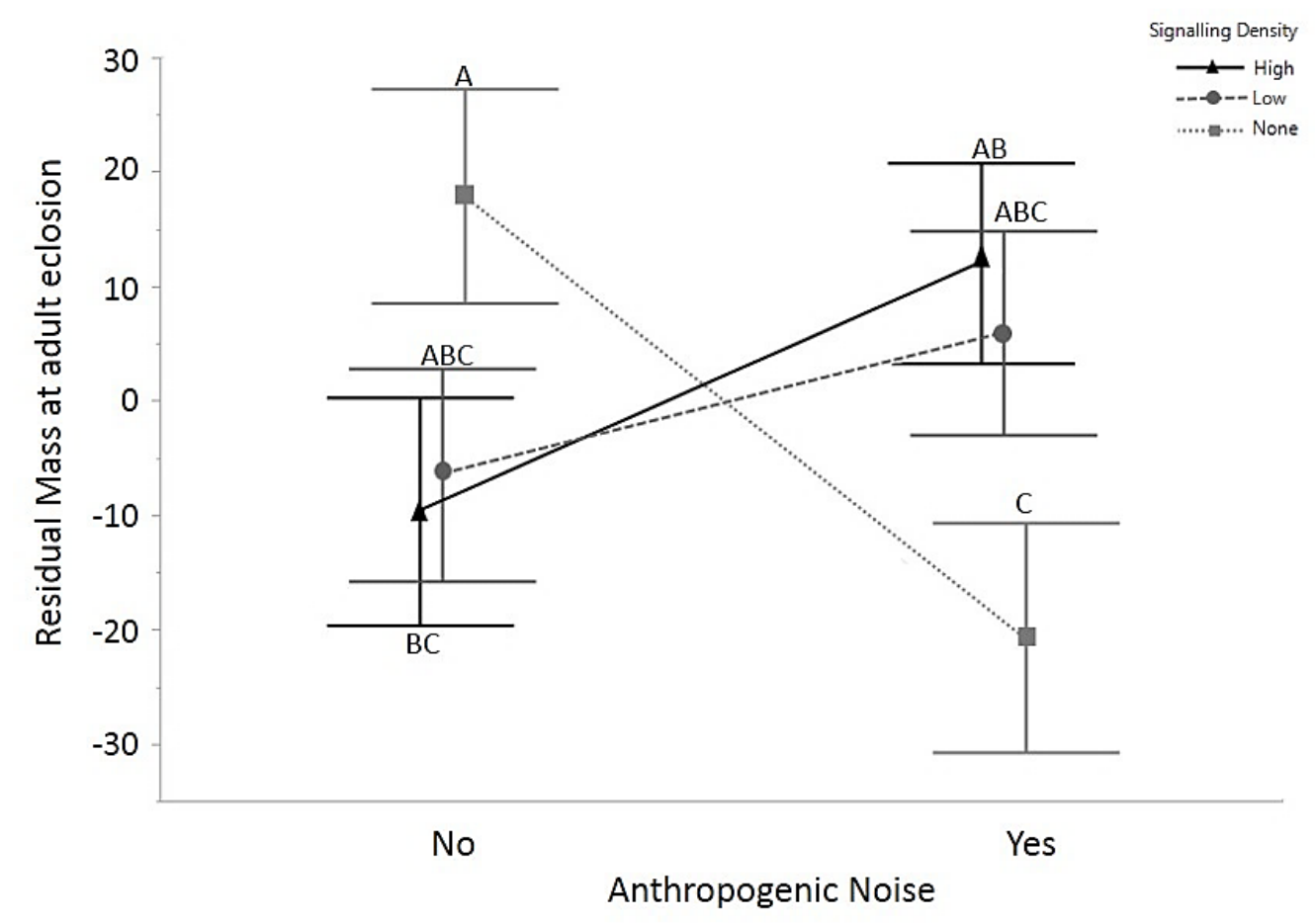

Figure 3.3. How the interaction between anthropogenic noise and the acoustic social environment during development influences female residual mass at adult eclosion. Bars are standard errors and letters denote significant differences. 


\subsubsection{Impact of Acoustic Treatment on Male Developmental Traits}

Male development time was significantly affected by the acoustic treatment $(P=$ $<0.0001$; Table 3.2; Figure 3.4). Crickets raised in the High and Low density acoustic social environments developed significantly faster than those raised in acoustic isolation (None). Specifically, males in the High and Low treatments developed wing pads 2 days sooner $($ High $\bar{x} \pm S E=30.7 \pm 0.51$; Low $\bar{x} \pm S E=30.8 \pm 0.51$; None $\bar{x} \pm S E=32.5 \pm$ 0.58 ) and reached adult eclosion 3 days sooner (High $\bar{x} \pm S E=49.1 \pm 0.6$; Low $\bar{x} \pm S E$ $=49.9 \pm 0.61 ;$ None $\bar{x} \pm S E=52.8 \pm 0.69)$ than males reared in the None treatment.

Male development time was also influenced by a significant interaction between Signalling density and Anthropogenic Noise $(P=0.0007$; Table 3.2; Figure 3.5). Specifically, males raised in the Low density treatment developed from wing pad eclosion to adult eclosion 2 days faster in the presence of anthropogenic noise $(\bar{x} \pm S E=$ $18.55 \pm 0.36$ days $)$ than without it $(\bar{x} \pm S E=20.21 \pm 0.39$ days $)$. Additionally, when anthropogenic noise was present, males in both the High and Low density treatment developed from wing pad to adult eclosion 3 days faster than male crickets raised in acoustic isolation (None treatment) (High $\bar{x} \pm S E=18.36 \pm 0.37$; Low $\bar{x} \pm S E=18.55$ \pm 0.36 ; None $\bar{x} \pm S E=21.22 \pm 0.39$ days $)$.

Neither male body size (PC1 size) nor residual mass at adulthood were significantly affected by the acoustic social environment (signalling density) treatment, the addition of anthropogenic noise, or the interaction between them (Table 3.2). 
Table 3.2. Results of the linear models examining effects of juvenile acoustic treatment during development on development time, body size (PC1 size) and residual mass at adulthood in males. Significant effects are bolded for both models and model effects, FDR $\mathrm{B}_{\mathrm{B}}$ y corrected $P=0.02(\mathrm{~N}=228)$. Parameter effects are included in Appendix 1, Table A1.2.

\begin{tabular}{|c|c|c|c|c|c|c|c|}
\hline Model & $\mathbf{R}^{\mathbf{2}}$ adj & $F_{\text {model }}$ & $P_{\text {model }}$ & Model Effect & DF & $\mathbf{F}$ & $\mathbf{P}$ \\
\hline \multirow[t]{4}{*}{ Hatching to Wing pad } & 0.03 & 1.96 & 0.05 & Season & 3 & 2.07 & 0.11 \\
\hline & & & & Signalling Density & 2 & 4.20 & 0.0163 \\
\hline & & & & Anthropogenic Noise & 1 & 0.02 & 0.88 \\
\hline & & & & Signalling Density*Anthropogenic Noise & 2 & 0.75 & 0.47 \\
\hline \multirow[t]{4}{*}{ Wing pad to Adult eclosion } & 0.18 & 7.04 & $<\mathbf{0 . 0 0 0 1}$ & Season & 3 & 4.41 & 0.0049 \\
\hline & & & & Signalling Density & 2 & 13.35 & $<\mathbf{0 . 0 0 0 1}$ \\
\hline & & & & Anthropogenic Noise & 1 & 1.41 & 0.24 \\
\hline & & & & Signalling Density*Anthropogenic Noise & 2 & 7.45 & 0.0007 \\
\hline \multirow[t]{4}{*}{ Hatching to Adult eclosion } & 0.11 & 4.61 & $<0.0001$ & Season & 3 & 2.47 & 0.06 \\
\hline & & & & Signalling Density & 2 & 11.14 & $<0.0001$ \\
\hline & & & & Anthropogenic Noise & 1 & 0.44 & 0.51 \\
\hline & & & & Signalling Density*Anthropogenic Noise & 2 & 3.83 & 0.02 \\
\hline \multirow[t]{4}{*}{ PC1 size } & 0.004 & 1.10 & 0.36 & Season & 3 & 0.31 & 0.82 \\
\hline & & & & Signalling Density & 2 & 0.86 & 0.42 \\
\hline & & & & Anthropogenic Noise & 1 & 0.81 & 0.37 \\
\hline & & & & Signalling Density*Anthropogenic Noise & 2 & 5.52 & 0.08 \\
\hline \multirow[t]{4}{*}{ Residual Mass } & -.004 & 0.88 & 0.53 & Season & 3 & 0.20 & 0.89 \\
\hline & & & & Signalling Density & 2 & 0.43 & 0.65 \\
\hline & & & & Anthropogenic Noise & 1 & 1.02 & 0.31 \\
\hline & & & & Signalling Density*Anthropogenic Noise & 2 & 2.09 & 0.13 \\
\hline
\end{tabular}




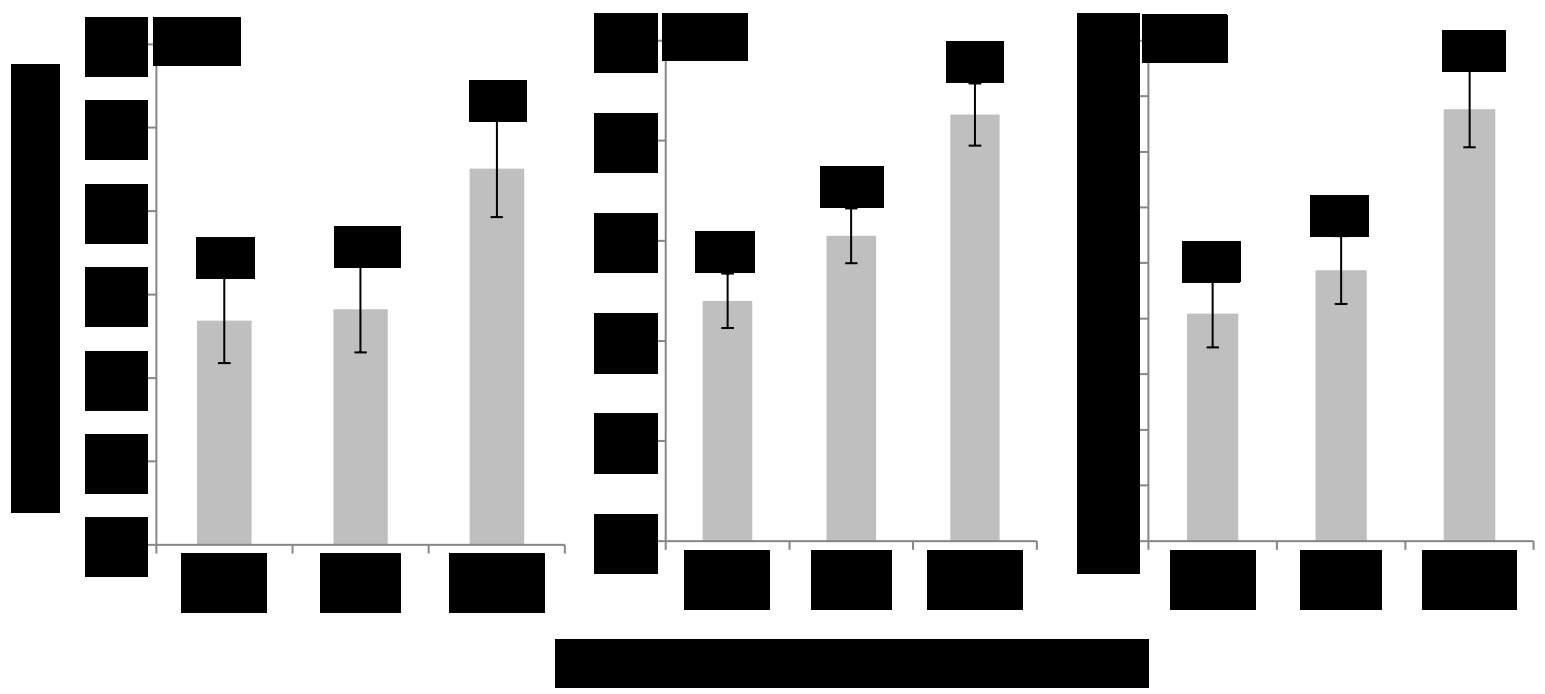

Figure 3.4. How the acoustic social environment during development influences male development time across the different life stages: (A) Hatching to Wing pad, (B) Wing pad to adult eclosion, and (C) Total Development Time. Error bars are standard errors and lowercase letters denote significant differences between acoustic treatment groups for each life stage. 


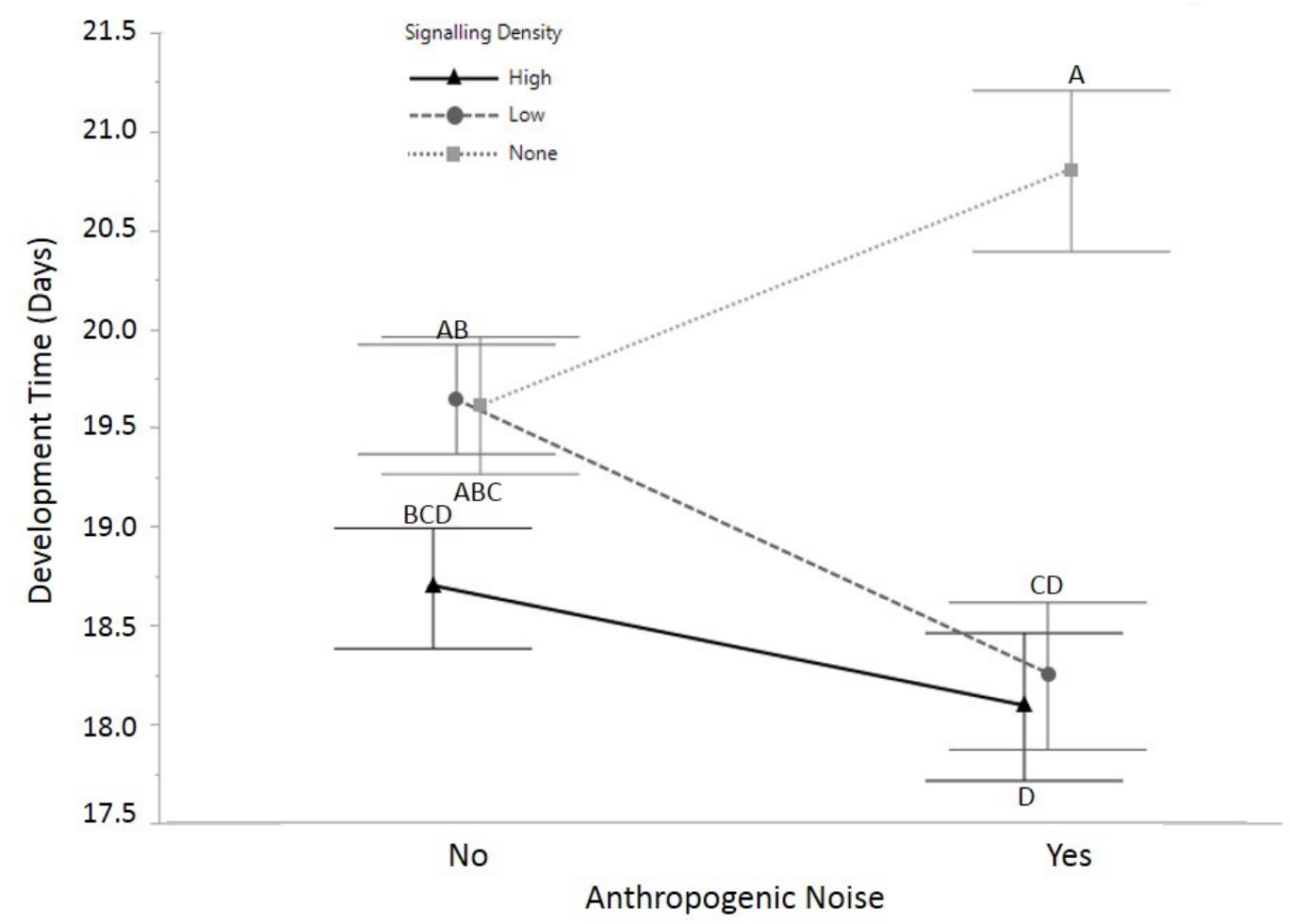

Figure 3.5. How the interaction between anthropogenic noise and the acoustic social environment during development influences male development time for Wing pad to adult eclosion. Bars are standard errors and letters denote significant differences. 


\subsubsection{Impact of Development Time on Adult Male and Female Morphology}

Female body size at adulthood was not influenced by development time. However, females that took longer to develop from wing pad to adult eclosion had higher residual mass than females developing more slowly $(P<0.0001$; Table 3.3).

Male body size at adulthood was influenced by development time. Specifically, males with larger body size developed faster, and the effect was stronger in the second half of development (from wing pad to adult eclosion; $P<0.0001$; Table 3). Conversely, males with higher residual mass took longer to develop from wing pad to adult eclosion than males with lower residual mass $(P=0.0002$; Table 3.3$)$. 
Table 3.3. Results of the linear models examining effects of development time on body size (PC1 size) and residual mass at adulthood in females $(\mathrm{N}=285)$ males $(\mathrm{N}=228)$. Significant effects are bolded for both models and model effects, FDR $\mathrm{B}-\mathrm{Y}$ corrected $P=0.027$.

Parameter effects are included in Appendix 1, Table A1.3.

\begin{tabular}{|c|c|c|c|c|c|c|c|}
\hline Model & $\mathbf{R}^{\mathbf{2}}$ adj & $F_{\text {model }}$ & Pmodel & Model Effect & DF & $\mathbf{F}$ & $\mathbf{P}$ \\
\hline \multicolumn{8}{|l|}{ Females } \\
\hline \multirow[t]{3}{*}{ PC1 size } & 0.02 & 1.66 & 0.15 & Season & 3 & 2.09 & 0.10 \\
\hline & & & & Days from Hatching to Wing pad & 1 & 0.22 & 0.64 \\
\hline & & & & Days from Wing pad to Adult Eclosion & 1 & 2.21 & 0.14 \\
\hline \multirow[t]{3}{*}{ Residual Mass } & 0.07 & 5.36 & 0.0001 & Season & 3 & 1.40 & 0.24 \\
\hline & & & & Days from Hatching to Wing pad & 1 & 4.98 & 0.03 \\
\hline & & & & Days from Wing pad to Adult Eclosion & 1 & 22.04 & $<0.0001$ \\
\hline \multicolumn{8}{|c|}{$e^{0}$} \\
\hline \multirow[t]{3}{*}{ PC1 size } & 0.16 & 8.78 & $<0.0001$ & Season & 3 & 0.30 & 0.0049 \\
\hline & & & & Days from Hatching to Wing pad & 1 & 5.18 & 0.0238 \\
\hline & & & & Days from Wing pad to Adult Eclosion & 1 & 31.98 & $<0.0001$ \\
\hline \multirow[t]{3}{*}{ Residual Mass Male } & 0.06 & 4.03 & 0.0016 & Season & 3 & 0.82 & 0.48 \\
\hline & & & & Days from Hatching to Wing pad & 1 & 2.20 & 0.14 \\
\hline & & & & Days from Wing pad to Adult Eclosion & 1 & 14.31 & 0.0002 \\
\hline
\end{tabular}




\subsection{Discussion}

The acoustic social environment juvenile G. pennsylvanicus experienced influenced their development time, size, and residual mass at adult eclosion. The presence of adult male acoustic signals during rearing resulted in faster development in both females and males and also resulted in larger adult females. While anthropogenic noise on its own did not influence these life history traits, anthropogenic noise did influence female residual mass and male development time via a significant interaction with signalling density. These results partially support my hypothesis that juvenile fall field crickets allocate resources during development to "match" their anticipated adult population compositions (Rodd et al. 1997; Stockley \& Seal 2001; Kasumovic et al. 2011).

In females, the acoustic social environment experienced during development, specifically exposure to adult male mate attraction signalling, resulted in a faster development time across both observed life stages. Juvenile females raised in the High and Low signalling density treatments may anticipate more mating opportunities upon adult eclosion than juvenile females reared with no available social information. In such environments, faster development times may lead to increased fitness when there are plenty of mating prospects. When mate availability is anticipated to be low, as in the social isolation (None) environment, developmental resource allocation is expected to shift away from growth rate towards attributes such as investments in reproductive tissues. Investment in extra eggs may allow females with fewer perceived mating opportunities to still have high fitness, provided they are able to find a mate. While my findings are similar to those reported by Kasumovic et al (2011) in Teleogryllus commodus, other studies investigating the effects of social environment during 
development in invertebrates have largely focused on males (Stockley \& Seal 2001; Kasumovic \& Andrade 2006; Bailey et al. 2010) or adult behaviour (Lierheimer \& Tinghitella 2017; Kasumovic et al. 2009; Bailey \& Zuk 2008). Thus, more longitudinal studies are needed to further elucidate whether my finding that juvenile females increase their growth rate when exposed to adult male signals can be generalized across species.

If females raised in the None environment allocated more resources towards reproductive tissue growth during development, then these females might also be larger adults, as adult body size and fecundity are often positively correlated in Orthoptera (Bertram et al. 2016; P. W. Bateman et al. 2001; Shoemaker 2003; Hedrick \& Kortet 2012; Carrière \& Roff 1995). However, my results revealed that females raised in the None environment were not larger upon adult eclosion than those from either the High or Low treatments. Further, while I did find a significant difference in body size between females raised in the High versus the Low environment, the High environment females were the largest, not the smallest.

An alternative explanation for my finding that females reared in the High density environment were larger is that they allocate more energy to growing larger to contend with potential male mate choice. In some cricket species, males preferentially court ( $G$. bimaculatus) (Bateman \& Fleming 2006a) and transfer more sperm (Acheta domesticus) (Gage \& Barnard 1996) to larger females. Thus, those females expecting more mate choice opportunities but also increased female competition may allocate resources to both developing faster and at larger, more attractive body sizes, to gain access to the abundance of available mates.

Females that were slower at developing from wing pad eclosion to adult eclosion 
had higher residual mass at adulthood than females that developed faster. Further, females reared without conspecific mate attraction signals (in the None treatment) were slower to mature, possibly shifting resources away from body size and investing more in reproductive tissue growth, resulting in a larger residual mass. In many arthropod species including crickets, however, the relationship between body size, residual mass and female fecundity, is not always well defined (Moya-Laraño et al. 2008; Honěk 1993; Whitman 2008; Kelly et al. 2014). While many previous studies have investigated how body size and residual mass relate to female fecundity, they often use dissimilar measurements for fitness, making it difficult to compare trends across research (Leather 1988). As I did not quantify reproductive tissue volumes, future studies should investigate whether perceived social environment influences fecundity.

Interestingly, residual mass in females was also influenced by an interaction between anthropogenic noise and the acoustic social environment that represented differences in male density. Specifically, females raised in the social isolation (None) environment had lower residual mass at adult eclosion when they were exposed to anthropogenic noise, compared to when they were not. Two things might explain this finding. First, as discussed above, females that developed in the None environment indicative of few or no available mates at adulthood might have invested heavily in reproductive tissues, resulting in high residual mass (significantly higher than females in the High acoustic environment treatment). However, when females developed in the None environment that was overlaid by anthropogenic noise, they may not have been capable of discerning the lack of signals from potential mates, and therefore may not have invested in the extra reproductive tissues or energy stores. A second, non-mutually 
exclusive hypothesis to explain this interaction between anthropogenic noise and social density is that the stress from anthropogenic noise might have affected the females' ability to gain weight, as has been found in several amphibian species (Iglesias-Carrasco et al. 2017) and marine invertebrates (De Soto et al. 2013). A review by Kight \& Swaddle (2011) revealed that anthropogenic noise caused changes to nearly all body systems across taxa. Aligning with my study, Kight \& Swaddle (2011) also showed that noise stress differentially affected females, and they suggested that noise-stressed individuals may not have the resources available for reproduction. Consequently, the lower body weight exhibited in G. pennsylvanicus females raised in the presence of anthropogenic noise and the absence of conspecifics may be attributed to lower reproductive tissue volume, compared to the other treatments.

Like females, males also developed faster across both observed life stages when they were exposed to adult male acoustic mate attraction signalling during development (High and Low treatments) than when they were raised in social isolation (None treatment). This is contrary to what I expected based on previous studies where juvenile males exposed to conspecific signalling took longer to develop, presumably to enable them to allocate the time and resources required to grow large and maintain high condition (Kasumovic et al. 2011; Bailey et al. 2010; DiRienzo et al. 2012). Juvenile males exposed to the adult male acoustic signals in the High and Low environments could have inferred an adult population dense in both males and females, thus causing them to mature faster as there would be plenty of mating opportunities upon adult eclosion. In the wild, G. pennsylvanicus are univoltine (one mating season each year), with egg diapause during the winter months. Further, the mating season is only a few 
months long in Ontario, running from mid-July to mid-October (Alexander 1968;

Bigelow 1962). Males raised in social isolation would face an adult population with few to no available females and might have taken longer to develop, instead allocating resources to sperm number, building signalling muscles, or reserves to fuel signalling muscles, as these males should have to signal more often or louder to attract elusive females. I test this signalling theory in Chapter 5 of my thesis.

Body size and mass have important fitness effects in adult male crickets, with larger males generally having a fitness advantage over smaller males. For example, larger males are more able to outcompete smaller males in aggressive encounters (fights) (Reaney et al. 2011; Briffa 2008; Judge \& Bonanno 2008), male size is positively correlated with attractive traits such as dominance (Savage et al. 2005; Loranger \& Bertram 2016; Bertram \& Rook 2012) and signalling quality (Bertram \& Rook 2012; Harrison et al. 2013), and females prefer to mate with larger males (P. W. Bateman et al. 2001; L. W. Simmons 1986; Saleh et al. 2014; Savage et al. 2005). However, I found no evidence that the acoustic social environment or anthropogenic noise influenced either adult body size or residual mass in males. Again, this is contrary to other studies which found that male crickets reared in the presence of adult male acoustic signals were both bigger and weighed more at adult eclosion compared to males reared in isolation (Kasumovic et al. 2011; Bailey et al. 2010; DiRienzo et al. 2012). I did, however, find a significant effect of development time on both adult body size and residual mass, with faster development times resulting in larger, heavier males. This suggests an indirect effect of my treatments, as males reared in the High density environment developed faster. Juvenile resource allocation is a complex process so adding additional variables to 
the rearing condition, such as diet manipulation, may aid in elucidating the subtle interactions of social environment with other factors affecting development (Whattam \& Bertram 2011; Kasumovic et al. 2011; Judge et al. 2008). For example, Kasumovic et al (2012) found that in male black field crickets (Teleogryllus commodus) the interaction of juvenile diet and acoustic social environment yielded different results than either factor alone. Normally, males develop quickly in the presence of low-quality adult male signals, as well as when fed a high protein diet (Kasumovic et al. 2011; Zajitschek et al. 2009), but when these factors interacted, males developed slower. Therefore, the indirect effects that I found in my study may be more reflective of the fact that juvenile social environment is only one of many contributing factors of resource allocation during development.

Male development time from wing pad to adult eclosion was influenced by a significant interaction between signalling density and anthropogenic noise. Specifically, males in the None treatment took three days longer, on average, to achieve their adult moult than males in the Low and High density treatments when there was Anthropogenic noise present. Conversely, when there was No Anthropogenic noise present, development time did not differ across signalling density treatments. This effect of Anthropogenic noise was the result of an increase in the development time of males in the None treatment combined with a decrease in the development time of males in the Low density treatment. This slower development time of males reared in social isolation might result from a masking or interfering effect of anthropogenic noise, making it a very uncertain environment for the developing males. Given the uncertainty of what they will experience as adults (uncertain competition or mates), males might increase reserves to 
be able to fuel the energetic requirements associated with fighting conspecific males or signalling conspecific females or improve immune function (and thus life-span) in an uncertain future. For example, in the Mediterranean field cricket (G. bimaculatus) smaller males had a higher encapsulation rate (a protective immune response) compared with larger males, providing smaller males with an immunological advantage (Rantala \& Roff 2005). An uncertain adult environment may thus result in developmental "bet hedging" where male developmental plasticity allows for multiple life history trajectories.

My study adds to the growing evidence that juveniles alter their developmental trajectory in response to their social environment and highlights the complexity of these plastic traits. My experimental design was intended to closely resemble signal density levels and anthropogenic noise in G. pennsylvanicus' natural habitat, while maintaining a highly controlled environment. Further confounding these results are a general lack of longitudinal evidence on how anthropogenic noise affects individuals across their lifetime (Kight \& Swaddle 2011). Up until now my thesis has focused on developmental plasticity. In Chapters 4-7 I explore the effects of behavioural plasticity in adulthood. 


\section{Chapter 4: Developmental acoustic social environment, but not anthropogenic noise, affects male field cricket aggression}

\subsection{Abstract}

Changes within the social environment have been found to alter aggression levels in males across several species. Increases in general adult population densities tends to lead to decreases in adult aggressive behaviour, while social isolation often causes more aggression. Juvenile social experience follows a similar pattern, with exposure to conspecifics during development leading to a decrease in adult aggression levels. Because the accurate assessment of signals is necessary for juveniles to determine the makeup of their future social environment, anthropogenic noise pollution that confounds the ability of individuals to accurately hear signals could have the potential to produce a significant effect on adult behaviour. I therefore examined changes in adult male aggressive behaviour in response to adult mate attraction signalling (the acoustic social environment) and urban noise experienced during development. I reared Focal male fall field crickets in physical isolation from other males while simultaneously exposing them to one of six different acoustic environments that differed in the social density of signalling adult males (High, Low and None) and anthropogenic noise (Present or Absent). As adults, I fought these Focal males against opponent males who were reared with conspecifics and assessed their aggressive behaviours. Regardless of the acoustic treatment they were raised in, Focal males won more fights than expected by chance and were more aggressive than Opponent males. While the developmental acoustic social environment or anthropogenic noise did not have any direct effect on the aggressive behaviour of Focal males, there were indirect effects. Juvenile's exposed to the High 
density male acoustic signalling treatment during development grew faster, and a faster development time resulted in larger males. Larger males, in turn, were more aggressive and won more fights than smaller males. Additionally, when I examined the behaviour of Focal males that lost fights, those that were raised in social isolation (None treatment) were more aggressive than losing males reared in the presence of adult male acoustic signals (High or Low treatments). Together, these findings support theoretical predictions that adult aggressive behaviour is partially dependent on the social environment experienced during development.

\subsection{Introduction}

The fitness of an individual depends on a match between its phenotype and the environment. Given this, changes within the environment can influence an individual's fitness, driving selection (Levins 1962; Via \& Lande 1985). Phenotypic plasticity, be it developmental or behavioural, allows animals to survive and reproduce in heterogenous environments. An important component of this heterogeneity is the social environment that the individual experiences (Kasumovic \& Brooks 2011; Pijanowski et al. 2011). Social cues can change throughout an individual's lifetime and may differentially affect both developmental and behavioural plasticity. For example, in both the Mediterranean ant (Cataglyphis cursor)(Clémencet \& Doums 2007) and the European ant (Temnothorax nylanderi)(Molet et al. 2017) adult worker size is positively correlated with colony size during development. Furthermore, differences in rival density and mate availability during development can also affect adult behaviour. Adult male dung flies (Scatophaga stercoraria), for example, exhibit altered mate searching behaviours in response to 
differences in larval density (Stockley \& Seal 2001). Additionally, adult male ocean field crickets (Teleogryllus oceanicus) that develop in silence as juveniles exhibit stronger satellite behaviour during playback of male signals than adult males that are exposed to acoustic long distance mate attraction signals as juveniles (Bailey et al. 2010). Importantly, developmental and behavioural plasticity can also overlap (Bretman et al. 2011). For example, in cases where investment in reproductive tissue, such as wing muscles in crickets, is constrained by development, acoustic mate attraction signalling effort could be reduced in adulthood. The ability to accurately predict the expected adult cohort during development can therefore result in fitness advantages (Kasumovic \& Brooks 2011).

One way of enhancing an individual's fitness is to maximize their likelihood of competitive success in aggressive contests. Aggressive contests are common in males of many species, and phenotypic traits are often strong predictors of competitive success. Numerous studies have shown that larger males, males in better condition, and/or males with larger weaponry/ornamentation often win aggressive contests (Andersson et al. 2002; Jennings et al. 2010; Rillich et al. 2007; Savage et al. 2005). The social environment that the individual experiences during development can influence these phenotypic traits that enhance competitive success. For example, male adult body size in guppies (Poecilia reticulata) and cichlid fish (Pelvicachromis taeniatus) is affected by social density during development, with higher densities resulting in larger males (Rodd et al. 1997; Hesse \& Thünken 2014). This same pattern has also been observed in field crickets (Gryllus integer)(DiRienzo et al. 2012), predatory mites (Phytoseiulus persimilis)(Schausberger et al. 2017), and the orb-web spider (Argiope bruennichi)(Cory 
\& Schneider 2017). Since the developmental social environment can affect life history traits which in turn can affect the outcomes of aggressive contests, the analysis of adult aggressive behaviour requires knowledge of whole life experience to avoid convolution of these separate influences.

In addition to development influencing adult body phenotype, aggressive behaviours can also be directly affected by the developmental social environment. For example, group reared male zebra finches (Taeniopygia guttata) are more aggressive than males reared in pair-groups (Bölting \& Von Engelhardt 2017). Similarly, in veiled chameleons, (Chamaeleo calyptratusoups) males reared in groups are more aggressive than males reared in social isolation (Ballen et al. 2014). Alternatively, male rats raised in isolation after weaning display increased and abnormal aggressive behaviours (Toth et al. 2011), a reaction also observed in Rhesus monkeys (Mitchell et al. 1966). Furthermore, male crickets (Gryllus integer) are less aggressive and less dominant when reared in the presence of acoustic male mate attraction signals indicative of large groups (DiRienzo et al. 2012). This result was similar to another study on field crickets (Gryllus bimaculatus), which showed that males reared in large groups are less aggressive in adulthood than males reared in isolation (Nagamoto et al. 2005). Juvenile crickets therefore appear capable of using acoustic cues during development to determine the expected composition of an adult population, including rivals, and modifying their development in such a way to result in specific phenotypes and behaviours at maturity (Olvido \& Mousseau 1995; Beckers \& Schul 2008; Kasumovic et al. 2009; DiRienzo et al. 2012).

The social environment during adulthood can also affect aggression levels, with increased densities often leading to reduced aggression. For example, adult male mice 
(Mus musculus) housed in groups fight less than males housed either in isolation or with females (Crawley et al. 1975). Females broiler chickens (Gallus gallus domesticus) have fewer aggressive interactions when placed in crowded pens compared with when they are introduced to open pen regions (Pettit-Riley et al. 2002). Further, the aggression levels of group attacks in the stingless bee (Trigona spinipes) are lower when the groups sizes are larger (Nieh et al. 2005). Male field crickets are also susceptible to changes in the adult social environment. In the wild, male field crickets maintain and defend territories from which they signal to attract females. Territory size varies between species and habitat, but intra-male distance can range from 1 to 50 meters (Hissmann 1991; French et al. 1986; Rowell \& Cade 1993; Cade 1981). As population density increases, the amount of time males spend searching for females increases, male satellite behaviour increases, and male-male encounters increase, while the amount of time that males spend signalling to attract females decreases, as does overall aggression levels (Hissmann 1991; French et al. 1986; Rowell \& Cade 1993; Cade 1981). Largely these behavioural shifts appear due to the increased cost of aggressive contests when competition for territory access is high. Conversely, when population densities are lower, aggression levels increase (Souroukis \& Cade 1993; Alexander 1961; French \& Cade 1989).

Aggressive behaviour and winning aggressive contests can influence male fitness in field crickets in several ways. In addition to gaining access to territories, female field crickets preferentially mate with more dominant males (Loranger \& Bertram 2016; L. Simmons 1986), prefer the chemical cues of dominant males (Kortet \& Hedrick 2005), and lay more eggs when mated to dominant males compared to subordinate males (Bretman et al. 2006). Female preference for more dominant males may also occur 
because dominant male crickets exhibit higher immunocompetence levels than subordinate males (Rantala \& Kortet 2004). Given the importance of aggressive interactions to male fitness, here I investigate how the juvenile acoustic environment of male North American fall field crickets, Gryllus pennsylvanicus, affects their aggressive behaviour in adulthood. Because the social system of field crickets is highly reliant on acoustic communication in the form of male long-distance signalling, courtship songs, and aggressive signals (Alexander 1961), field crickets rely heavily on acoustic communication to garner information about the male composition of the current and future social cohort, including density and male fitness (Otte 1974). In Chapter 3 I showed that information in the social environment directly influenced male development time and indirectly influenced body size. Here I investigate whether acoustic information about their social environment during development has the potential to influence adult aggressive behaviour.

In addition, I examined if and how the presence of anthropogenic noise, specifically urban noise pollution, interferes with the crickets' ability to properly assess the expected adult cohort, resulting in a shift in aggressive behaviour upon maturity. Human habitation can greatly alter the acoustic structure of the environment, resulting in a phenotypic change in the acoustic communication of many species living in the surrounding area (Pijanowski et al. 2011; Laiolo 2010). Urban noise can affect acoustic communication by causing shifts in amplitude, frequency, as well as temporal shifts, as observed in birds and amphibians (Luther \& Derryberry 2012; Laiolo 2010). While relatively few studies have investigated the effects of anthropogenic noise on the communication systems of terrestrial invertebrates, exposure to anthropogenic noise can 
affect taxonomic diversity (Bunkley et al. 2017), alter signal production in grasshoppers (Chorthippus biguttulus)(Lampe et al. 2012; Lampe et al. 2014), tree crickets (Oceanthus pellucens)(Orci et al. 2016), and cicadas (Cryptotympana takasagona)(Shieh et al. 2012), and impede female phonotaxis in crickets (Gryllus bimaculatus) (Schmidt et al. 2014). Like much of the current research on anthropogenic noise, however, the majority of these studies were limited to investigating how anthropogenic noise impacts changes in acoustic communication of adults. Research exploring how exposure to anthropogenic noise during juvenile development influences developmental plasticity or adult aggressive behaviour has been more limited.

To address how the juvenile acoustic environment of male G. pennsylvanicus affects adult aggressive behaviour, I isolated developing males into individual containers and then broadcasted sounds to them represented different levels of adult male densities and anthropogenic noise. Once males reached adulthood I continued to expose them to their acoustic environment soundtrack for another week. I then competed them against rival opponents in aggression contests. Males who expect high male-male competition may strategically allocate resources to growth and body size at adulthood. Further, they may reduce aggressive behaviour in an attempt to alleviate costs associated with high competition such as injury (due to increased aggressive interactions) or increased predation risk (DiRienzo et al. 2012). I therefore predicted males reared in the acoustic social environment representing a high density of adult males would be larger and exhibit less aggressive behaviour upon maturity than males reared in a low density acoustic environment. I also predicted that anthropogenic noise pollution would mask the acoustic signals, making it difficult for juveniles to accurately predict the composition of a future 
adult population and resulting in elevated aggression due to lower expected male-male competition.

\subsection{Methods}

\subsubsection{Study Species and Cricket Rearing}

I used laboratory-reared North American Fall Field crickets, G. pennsylvanicus, in my study. I randomly assigned each one-week old cricket to one of 6 acoustic social environment treatments and placed them into a larger communal treatment bin. The communal treatment bins and the lid were lined with acoustic foam to prevent sound contamination across treatments. Each communal treatment bin lid contained 4 speakers for playback of the assigned acoustic social environment soundtracks. I used 6 different social acoustic soundtrack treatments in a $3 \times 2$ factorial design: 3 with different densities of male acoustic signals, and 3 with these male acoustic signals overlaid with anthropogenic noise. Crickets were kept on a 14h:10h light:dark photoperiod at $28 \pm 2$ ${ }^{\circ} \mathrm{C}$. Thrice weekly, each juvenile cricket had its food and water replenished and was checked for wing pad eclosion or adult eclosion.

Once these experimental crickets reached adult eclosion, they were removed, given an experimental ID, weighed (Pioneer Balance model PA214, OHAUS Corporation, USA), placed in a new individual container $(\mathrm{L} \times \mathrm{W} \times \mathrm{H}=10 \times 7 \times 7 \mathrm{~cm})$, and returned to their same acoustic social environment treatment. The experimental crickets were therefore reared in physical isolation and experienced their assigned acoustic environment treatment from 1 week post hatch until they were run in their behavioural trials as adults.

Although mean age for signalling in G. pennsylvanicus is 6-7 days of adulthood, 
males can produce an acoustic signal as early as 3-4 days post-imaginal moult (Cade \& Wyatt 1984; Judge 2011). I therefore housed adult experimental males in separate acoustic social environment treatment bins to ensure no interference with the juvenile acoustic social environment treatments. Furthermore, I housed a maximum of 6 adult males together at any one time to ensure that any potential extra signals produced by these males was kept at a low level. These males also remained in individual containers to ensure that they did not experience any aggressive encounters before behavioural trials. At 7 days post-imaginal moult, I subjected the adult males to behavioural trials consisting of aggression and signalling trials (information on the signalling trials is provided in Chapter 5). Given aggressive contests necessarily involve two individuals, I termed the experimental males reared in the different acoustic social environment treatments the "focal males" and the males reared to be their aggressive partners the “opponent males”.

Please see Chapter 2 for a detailed description of my common general protocol.

\subsubsection{Rearing of Opponent Males}

Opponent males for use in the aggression contests where chosen at random from the laboratory colony. These males were separated from the colony bins and placed into a communal "opponent male" rearing bin shortly after wing-bud eclosion (as soon as sex could be appropriately assigned). Opponent males were provided with the same food, temperature, and light schedule as focal males (males that were reared in the different acoustic social environment treatments). The communal opponent male rearing bin was kept in the same greenhouse room as the main cricket colony and so experienced the acoustic environment of free calling male crickets and general colony maintenance noise. 
I checked developing opponent males for adult eclosion thrice weekly. Once the opponent male eclosed to adulthood, I placed him into his own container $(500 \mathrm{ml})$ with food, water, a crumpled piece of paper towel for shelter, and a screened lid for ventilation. Containers housing individual adult opponent males were kept in the colony greenhouse until they were $7-9$ days post-adult eclosion and needed for an aggression trial. After the aggression trial, opponent males were euthanized via freezing, photographed, and measured in an identical manner to focal males.

\subsubsection{Male Aggression Trials}

On days 6 post imaginal moult, prior to aggression trials, focal males were age and weight matched to an opponent male to within 5 days of age and $25 \%$ body difference (Pioneer Balance model PA214, OHAUS Corporation, USA). As weight differences may effect fight outcome (Hofmann \& Schildberger 2001; Savage et al. 2005), the mean weight difference between partners (a focal male and opponent male duo) was kept as low as possible ( $\bar{x}=7.6 \%$ difference). However, due to a temporary shortage of opponent males, 9 (of 201, <5\%) partner pairs ended up with a weight difference between 20-25\%. To distinguish between males during an aggression trial, both the focal and opponent male had their pronotums painted in contrasting colours using nail polish (Sally Hansen Insta-Dri).

Aggression trials took place 7 - 9 days post imaginal moult in an acoustically isolating chamber $(142 \times 79 \times 96.5 \mathrm{~cm}, \mathrm{~L} \times \mathrm{W} \times \mathrm{H})$ to limit observer disturbance. The trials took place between $08: 00$ and $17: 00 \mathrm{H}$, at $21-27^{\circ} \mathrm{C}\left(\mathrm{SE}+/-0.04^{\circ} \mathrm{C}\right)$. The chamber was illuminated using low intensity LED light sources (3.6W, AC120V). Prior to each trial, the partners (one focal and one opponent) were placed in a plexiglass arena (15.5 x 
$15.5 \times 21 \mathrm{~cm}, \mathrm{~L} \times \mathrm{W}$ X H) with a sand bottom, separated by an opaque plexiglass partition (Figure 4.1). After a 5-min acclimatization period, the partition was removed and the two males freely interacted for 20 minutes. The trials were recorded from $22 \mathrm{~cm}$ above the top of the arena using a GoPro HERO4 Silver camera (GoPro Inc. USA), remotely controlled on a tablet or smart phone. Aggressive behaviours and dominance was scored following the protocol outlined below in the section on scoring aggressive trials. Between each trial the sand floor was raked, and the arena walls were wiped down using a 95\% ethanol solution. The sand was replaced every 5-10 trials. 


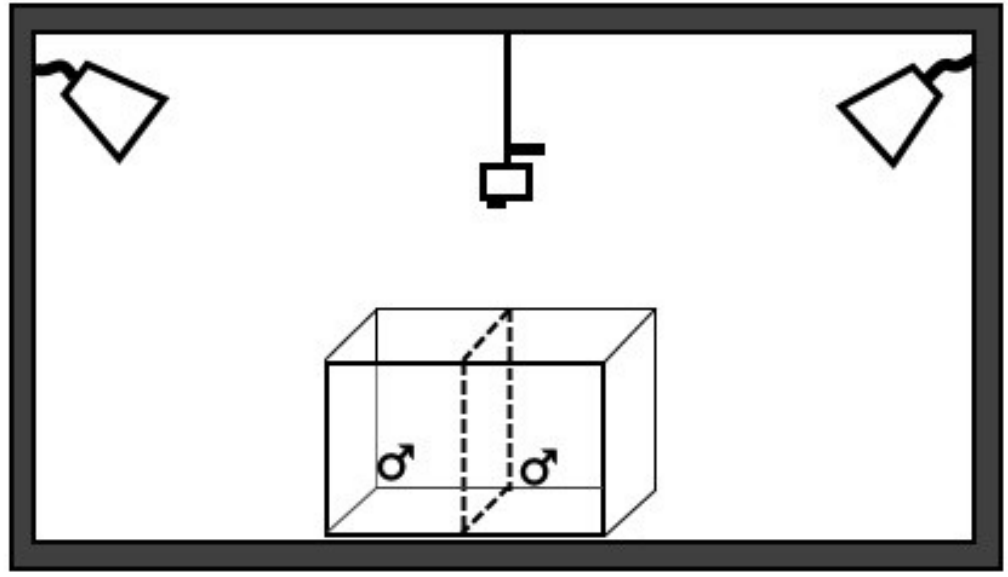

Figure 4.1. Experimental design set up showing the sound-attenuating chamber, trail arena, and GoPro HERO4 camera. During trials this chamber was closed, and trials were observed via the camera to limit disturbance of the males. 


\subsubsection{Scoring Aggression Trials}

Dominance in field crickets is usually determined through the outcomes of aggressive interactions. Alexander (1961) described five "levels" of aggression interactions: "Level 1: Contact terminated without clear dominance, no apparent retreat, and no apparent aggression; Level 2: Contact terminated by retreat without apparent aggression; Level 3: Contact terminated by retreat after mild to moderate one-sided aggression or mild reciprocal aggression; Level 4: Contact terminated after moderate to intense reciprocal aggression; and Level 5: Contact terminated only after sustained combat". The dominantsubordinate relationship is easily observed after a fight as the subordinate male (loser) displays avoidance behaviour towards the dominant male (winner). Further, the winner often also performs a victory acoustic signal and juddering display (Alexander 1961). I watched videos and scored the aforementioned aggressive behaviours using the open source event logging software BORIS (version 6.2.2) (Friard \& Gamba 2016), which allows for the computation of the timing, duration, and frequency of all aggressive behaviours. I scored the following behaviours on a scale from 0 to 6 (adapted from Adamo \& Hoy, 1995; Bertram \& Rook, 2012; Jang, Gerhardt, \& Choe, 2008):

$$
\begin{aligned}
& 0=\text { no aggression/withdrawal } \\
& 1=\text { unilateral or bilateral antennal fencing } \\
& 2=\text { kick } \\
& 3=\text { unilateral mandible flare, aggressive call, chase, or bite (mandible engagement) } \\
& 4 \text { = bilateral mandible flare, aggressive call, chase, or bite (mandible engagement) } \\
& 5 \text { = bilateral grapple: two males lock mandibles and pull or wrestle; }
\end{aligned}
$$


$6=$ victory display (body judder with/without call)

Using these scores, I quantified 3 measures of aggression for each focal and opponent male: maximum aggression score, aggression intensity score, and relative aggression score. Maximum aggression score was the highest scoring behaviour recorded for the male (0-6). Aggression intensity score was a cumulative measure calculated by multiplying each behavior's aggressive score by the duration of the behaviour. These weighted scores were then summed across all aggressive behaviours for each male and then divided by trial duration time (e.g., $1200 \mathrm{sec}$ ) times the maximum score possible (e.g., 6) (Equation 4.1).

\section{Equation 4.1:}

$$
\text { Aggression Intensity Score }=\frac{\Sigma \text { score of behaviour } \mathrm{X} \text { duration of behaviour }}{\text { trial length } \mathrm{X} \text { maximum possible aggression score }}
$$

Relative aggression score was the aggression score of each focal male relative to the aggression score of his opponent. Focal and opponent males were designated as fight winners or losers based on their dominance behaviour (victory displays: acoustic signals and juddering behaviour) and the number of retreats of the loser (adapted from Bertram, Rook, Fitzsimmons, \& Fitzsimmons, 2011). All aggression trials took place using animals that eclosed between September 2015 and June 2017.

\subsubsection{Statistics}

All statistical analyses were completed using JMP statistical software (version 11.0.0 SAS Institute). To determine adult residual mass and body size at adult eclosion, I 
combined body size measurements using a principle component analysis (PCA) to extract orthogonal vectors from pronotum length, pronotum width, and head width. The first principal component ( $\mathrm{PC} 1$ size) explained $92 \%$ of the variation in male size (Eigenvalue $=2.8$ ); all size measures loaded equally on PC1 size (eigenvectors ranged from 0.57 to 0.59). I then regressed body size (PC1 size) against body weight to determine residual mass at adult eclosion.

Following the statistical protocols set out in Chapter 3, I used linear models (LMs) to examine the effects of the acoustic social environment experienced on their development time, adult body size (PC1 size), and adult residual mass. I completed these statistical analyses again as these were different males than I used in Chapter 3 and I wanted to ascertain whether the acoustic environment they experienced during development had similar effects as it did in Chapter 3. Further, given the importance of body size on winning aggressive fights in crickets, I felt it important to determine how social environment during development influenced body size at adulthood. For these LMs the dependent variables were days from hatching to Wing pad eclosion, days from Wing pad eclosion to adult eclosion, days from Hatching to Adult eclosion (total development time), adult body size (PC1 Size), and adult residual mass. The independent variables included in each LM were signalling density (High, Low, None), anthropogenic noise (Yes, No), and their interactions (signalling density*anthropogenic noise). I also ran a separate LM to explore how development time on its own affected body size (PC1 size) and residual mass at adulthood. The independent variables were days from hatching to wing pad eclosion and days from wing pad eclosion to adult eclosion. Because I ran my experiment over a 21- month period, I also included season of hatching (Winter, Spring, 
Summer, Fall) as a covariate to control for possible block effects.

Using principal components analysis, I identified a single principal component $(\mathrm{PC} 1$ aggression $)$ that explained $73 \%$ (eigenvalue $=2.19)$ of the variation in the three measures of aggression: maximum aggression score, aggression intensity score, and relative aggression score. All three measures of aggression loaded equally and positively onto PC1 aggression (eigenvectors ranged from 0.54 to 0.61 ; Table 4.1 ). I used this PC1 aggression as the measure of aggression in all subsequent statistical analyses that included aggression. 
Table 4.1. PCA factor loading values for aggression scores. All loadings were positively associated with the aggression facors.

\begin{tabular}{lr}
\hline Fine Scale Signal Component & PC1 (aggression) \\
\hline Eigenvalue & 2.19 \\
Percent & 72.95 \\
Cumulative Percent & 72.95 \\
Maximum aggression score & 0.539 \\
Aggression intensity score & 0.581 \\
Relative aggression intensity score & 0.610 \\
\hline
\end{tabular}


I used an exact goodness of fit test to determine if focal males differed from opponent males in their probability of winning the fights. I used a linear model (LM) to determine if the acoustic social environment influenced aggression or if focal males differed from opponent males in their aggressive behaviour. The independent variables were signalling density (High, Low, None) in the acoustic social environment, anthropogenic noise (Yes, No) in the acoustic social environment, the interaction between these two variables, individual weight before the aggression trial, subject type (focal vs. opponent) and season (Winter, Spring, Summer, Fall).

I ran a generalized linear model (GLM) with binomial distribution and CompLog Log link function to quantify the factors influencing the likelihood of focal males winning or losing their fight. The independent variables were the signalling density (High, Low, None) and anthropogenic noise (Yes, No) in the acoustic social environment the focal male was reared in, the interaction between signalling density and anthropogenic noise, percentage weight difference between trial partners, the focal male's adult body size (PC1 Size), the focal male's residual mass, and season (Winter, Spring, Summer, Fall). The dependent variable was categorical (winner or loser). I also ran a LM to examine the effects of the aforementioned independent variables on the focal males' aggressive behaviour. Here I used PC1 aggression as the dependent variable. To determine if there was a difference in aggressive behaviour between winners and losers, I ran the LM twice, once for focal male winners and once for focal male losers.

I tested all models for violations of normality by examining the distribution of residual diagnostic plots (residuals vs fitted values and Q-Q plots); I also tested models for violations of homoscedasiticy by looking for dependency between the residuals and 
the fitted values. Normality and homoscedasticity assumptions were not violated. Pvalues were corrected using false the discovery rate FDR $_{\mathrm{B}-\mathrm{Y}}$ method (Benjamini \& Yekutieli 2001).

\subsection{Results}

I reared 228 juvenile males to adulthood across the 6 different acoustic social environments. I was unable to quantify aggressive behaviour in 23 of these focal males because I was unable to weight match them with an appropriate partner. A further 4 focal males were not included in the analyses because either they did not move during the 20 min trial $(\mathrm{N}=2)$, their fight video was lost $(\mathrm{N}=1)$, or the focal male's acoustic social environment soundtrack was accidentally switched prior to the fight $(\mathrm{N}=1)$. I therefore quantified aggressive behaviour of 201 focal males (High density: Anthropogenic noise 31, No Anthropogenic noise 28; Low density: Anthropogenic noise 32, No Anthropogenic noise 30; Isolation: Anthropogenic Noise 31, No Anthropogenic noise 28). After analysis of their aggressive behaviours, a small subset of males $(\mathrm{N}=4)$ were removed as outliers because their relative aggression score could not be calculated. This resulted in a total of 197 aggressive fights being analyzed and included below.

\subsubsection{Impact of Acoustic Treatment on Male Aggressive Behaviour}

Focal males (reared alone with the acoustic social environment treatment soundtrack broadcasted during development and into adulthood) were twice as likely than their opponents (reared in a group exposed to acoustic signals of the rearing colongy) to win the fight: focal males won $65 \%$ of their fights while their opponents only won $35 \%$ of their fights (Exact test of goodness of fit, $P<0.0001$ ). Focal males were also more 
aggressive than their opponents $(P<0.0001$; Table 4.2).

When just examining focal males, body size (PC1 size) influenced the likelihood the male would win his fight ( $P=0.0190$; Table 4.3) and the aggressive level of the fight $(P=0.0050 ;$ Table 4.4). Specifically, larger focal males were more likely to win their fight and were more aggressive than smaller focal males (Figure 4.2). The aggressiveness of the fight and the probability of winning the fight was not influenced by the developmental acoustic social environment treatment, residual mass, or the percent difference in body weight between opponents (Tables 4.3, 4.4). 
Table 4.2. Results of the linear model examining effects of acoustic treatment, weight $(\mathrm{mg})$ of males before the trial, and subject of male (focal vs opponent) on aggression levels. Significant effects are bolded. FDR $\mathrm{BY}_{\mathrm{B}}$ corrected level of significance is $P=0.0219$ $(\mathrm{N}=394)$. Parameter effects are included in Appendix 2, Table A2.1.

\begin{tabular}{lcrrrrrr}
\hline Model & $\mathbf{R}^{2}$ adj & $\mathbf{F}_{\text {model }}$ & $\mathbf{P}_{\text {model }}$ & Model Effect & DF & F & P \\
\hline PC1(aggression) & $\mathbf{0 . 0 9}$ & $\mathbf{4 . 7 0}$ & $\mathbf{< 0 . 0 0 0 1}$ & Season & 3 & 0.22 & 0.88 \\
& & & & Signalling Density & 2 & 1.31 & 0.11 \\
& & & & Anthropogenic Noise & 1 & 0.03 & 0.90 \\
& & & & Signalling Density*Anthropogenic Noise & 2 & 1.22 & 0.16 \\
& & & & Weight Before Trial (mg) & 1 & 0.02 & 0.45 \\
& & & & Subject (Focal Male vs Opponent Male) & $\mathbf{1}$ & $\mathbf{3 9 . 6 7}$ & $<\mathbf{0 . 0 0 0 1}$ \\
\hline
\end{tabular}


Table 4.3. Results of the generalized linear model on focal males examining effects of acoustic treatment, percentage difference in body weight between fighting males, body size of focal males (PC1 size), residual mass of focal males, and season on likelihood of being a winner or loser. Significant effects are bolded. FDR $\mathrm{BY}_{\mathrm{B}}$ corrected level of significance is $P=0.0193(\mathrm{~N}=197)$. Parameter effects are included in Appendix 2, Table A2.2.

\begin{tabular}{llrrr}
\hline Model & Model Effect & DF & $\mathbf{X}^{\mathbf{2}}$ & $\mathbf{P}$ \\
\hline Win/Lose & Season & 3 & 3.27 & 0.35 \\
& Signalling Density & 2 & 0.91 & 0.63 \\
& Anthropogenic Noise & 1 & 0.16 & 0.69 \\
& Signalling Density*Anthropogenic Noise & 2 & 3.90 & 0.14 \\
& \% Difference in Partner Weight & 1 & 2.17 & 0.14 \\
& PC1 (size) & $\mathbf{1}$ & $\mathbf{5 . 0 1}$ & $\mathbf{0 . 0 1 9 0}$ \\
& Residual Mass & 1 & 1.32 & 0.25 \\
\hline
\end{tabular}


Table 4.4. Results of the linear models on focal males examining effects of acoustic treatment, percentage difference in body weight between fighting males, body size of focal males (PC1 size), and season on aggression (PC1 aggression). Significant effects are

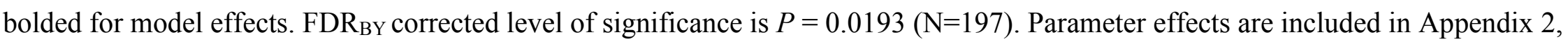

Table A2.3.

\begin{tabular}{lrrrrrrr}
\hline Model & $\mathbf{R}_{\text {adj }}{ }^{2}$ & $\mathbf{F}_{\text {model }}$ & P $_{\text {model }}$ & Model Effect & DF & F & P \\
\hline PC1 (aggression) & 0.05 & 1.98 & 0.03 & Season & 3 & 1.10 & 0.35 \\
& & & & Signalling Density & 2 & 0.21 & 0.81 \\
& & & & Anthropogenic Noise & 1 & 0.06 & 0.81 \\
& & & & Signalling Density*Anthropogenic Noise & 2 & 0.28 & 0.76 \\
& & & & \% Difference in Partner Weight & 1 & 3.32 & 0.07 \\
& & & & PC1 (size) & $\mathbf{1}$ & $\mathbf{8 . 0 7}$ & $\mathbf{0 . 0 0 5 0}$ \\
& & & & Residual Mass & 1 & 2.93 & 0.09 \\
\hline
\end{tabular}




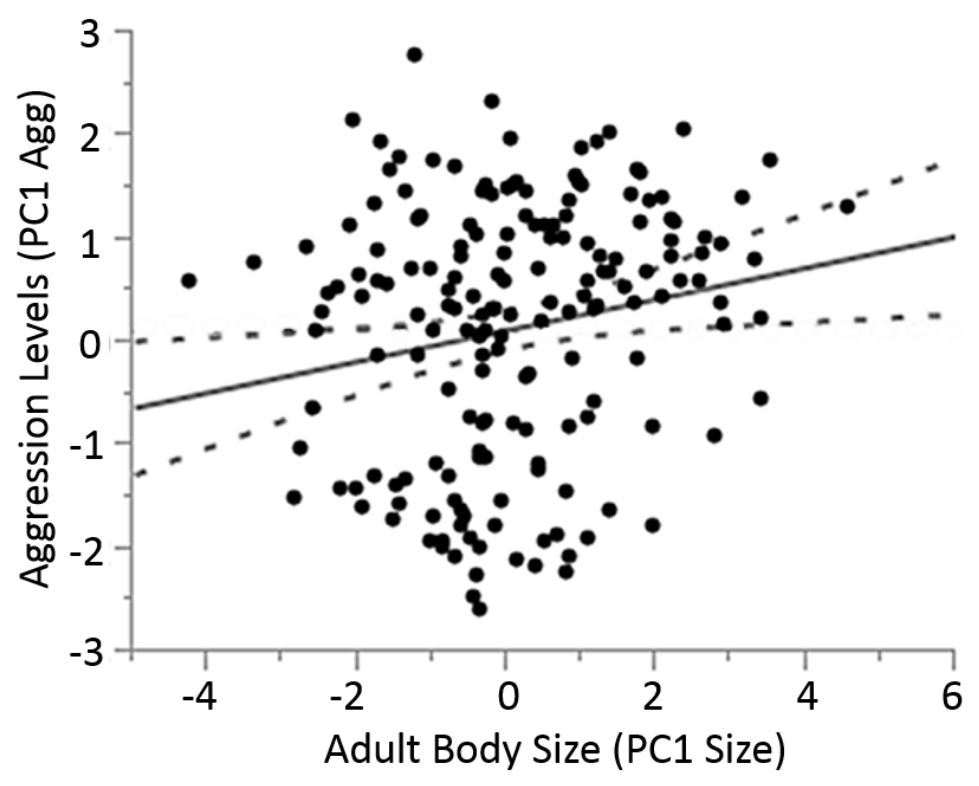

Figure 4.2. Leverage residual plot showing the effects of adult body size (PC1 size) on aggression levels were negative values are less aggressive. The solid line indicates the relationship between body size and signalling component after all other variables in the model has been accounted for; the dashed lines represent $95 \%$ confidence intervals. 
Fight losers were not as aggressive as fight winners. However, the aggression levels of losers were significantly affected by the acoustic social environment treatment they were exposed to during development and adulthood. Specifically, losers that developed in the None environment (isolation) were significantly more aggressive compared to losers that developed in either the High or Low density environments $(P=$ 0.0035; Table 4.5, Figure 4.3). The aggression level of winners was not affected by winner's developmental acoustic environment. 
Table 4.5. Results of the linear models examining effects of acoustic treatment, percentage difference in body weight between trial partners, body size of focal males (PC1 size), and season on aggression (PC1 aggression) in focal males for losers and winners.

Significant effects are bolded. $F_{B} R_{B Y}$ corrected level of significance is $\mathrm{P}=0.0193\left(\mathrm{~N}_{\text {losers }}=67, \mathrm{~N}_{\text {winners }}=130\right)$. Parameter effects are included in Appendix 2, Table A2.4.

\begin{tabular}{lrrrlrrr}
\hline Model & $\mathbf{R}^{2}{ }_{\text {adj }}$ & $\mathbf{F}_{\text {model }}$ & $\mathbf{P}_{\text {model }}$ & Model Effect & DF & $\mathbf{F}$ & $\mathbf{P}$ \\
\hline PC1 (aggression) & $\mathbf{0 . 2 3}$ & $\mathbf{2 . 7 8}$ & $\mathbf{0 . 0 0 6 2}$ & Season & 3 & 0.27 & 0.85 \\
Losers & & & & Signalling Density & $\mathbf{2}$ & $\mathbf{6 . 2 3}$ & $\mathbf{0 . 0 0 3 5}$ \\
& & & & Anthropogenic Noise & 1 & 0.01 & 0.91 \\
& & & & Signalling Density* Anthropogenic Noise & 2 & 1.29 & 0.28 \\
& & & & \% Difference in Partner Weight & 1 & 2.13 & 0.15 \\
& & & & PC1 (size) & 1 & 0.69 & 0.41 \\
& & & & Residual Mass & 1 & 2.10 & 0.15 \\
& & \multirow{2}{*}{0.59} & Season & 3 & 0.38 & 0.76 \\
PC1 (aggression) & -0.01 & 0.85 & & Signalling Density & 2 & 0.98 & 0.38 \\
Winners & & & & Anthropogenic Noise & 1 & 0.50 & 0.48 \\
& & & & Signalling Density*Anthropogenic Noise & 2 & 0.76 & 0.47 \\
& & & & \% Difference in Partner Weight & 1 & 0.12 & 0.73 \\
& & & & PC1 (size) & 1 & 0.97 & 0.33 \\
& & & & Residual Mass & 1 & 1.95 & 0.16 \\
\hline
\end{tabular}




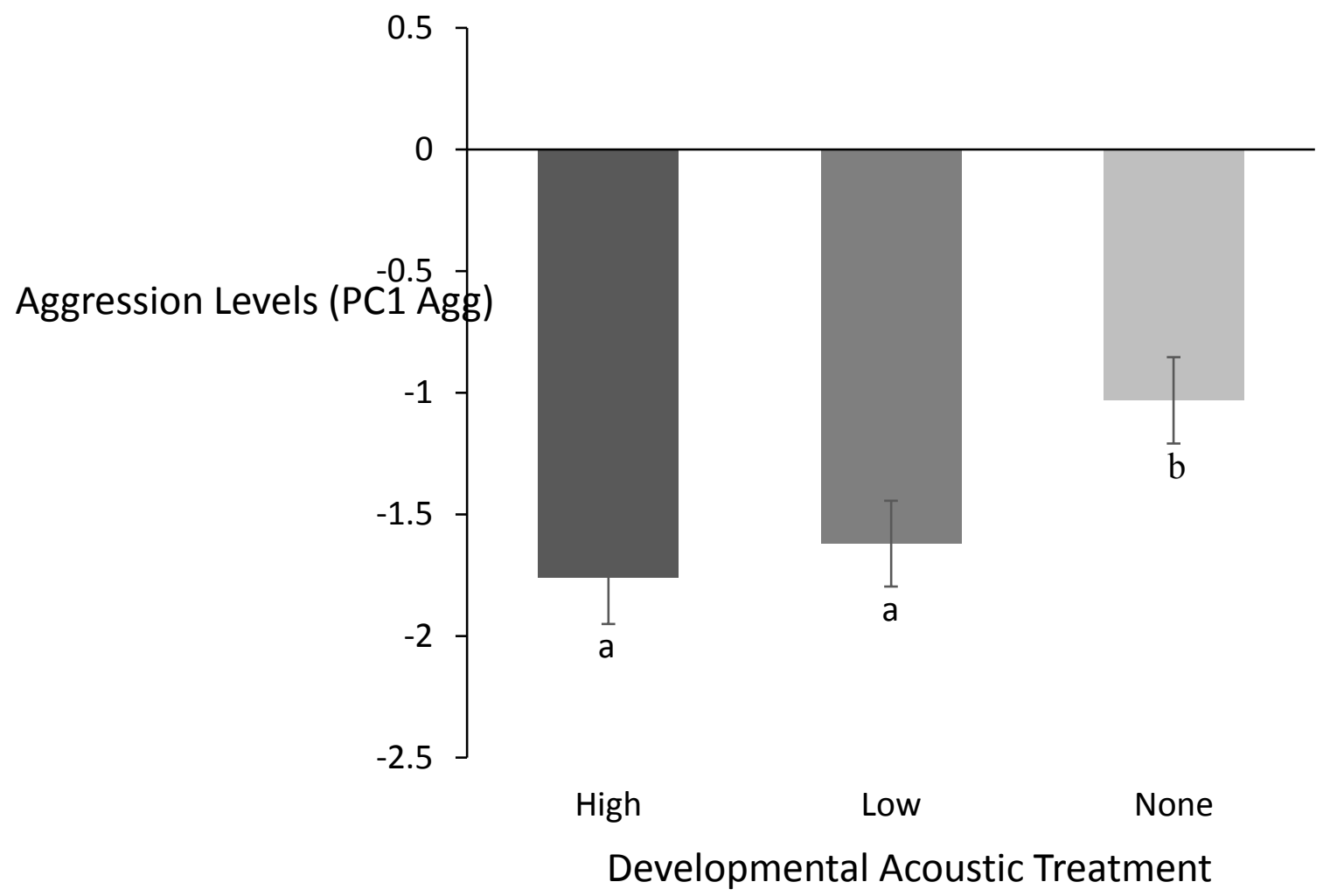

Figure 4.3. The effects of developmental acoustic treatment (Signalling Density) on Aggression levels in losing males. Negative values are less aggressive. Bars are standard errors and letters denote significant differences. 


\subsubsection{Impact of Acoustic social environment Treatment on Life History Traits}

Because the males in this chapter are different from the males I used in Chapter 3, I also repeated the analyses I conducted in Chapter 3 to determine how acoustic treatment influenced developmental life-history traits. Male development time was significantly affected by the acoustic treatment (Table 4.6). Males raised in the High density acoustic social environment developed wing pads approximately 2 days sooner than males in the Low density acoustic social environment, and approximately 4 days sooner than males raised in the None acoustic social environment $(P<0.0001$, High $\bar{x} \pm S E=32.03 \pm 0.66$, Low $\bar{x} \pm S E=34.15 \pm 0.68$, None $\bar{x} \pm S E=36.70 \pm 0.70)$ (Table 4.1, Figure 4.4 A). Males raised in the High density acoustic social environment also reached adult eclosion approximately 2 days sooner than males in the Low density and 6 days sooner than males in the None acoustic social environment $(P=0.021$, High $\bar{x} \pm S E=52.17 \pm 0.79$, Low $\bar{x} \pm S E=54.86 \pm 0.82$, None $\bar{x} \pm S E=58.17 \pm 0.83$ ) (Table 4.6, Figure 4.4 C). Total development time (hatching to adult eclosion) was also influenced by a significant interaction between Signalling Density x Anthropogenic Noise $(P=0.0086$; Table 4.6). Males raised in the None acoustic social environment treatment developed from hatching to adult eclosion significantly faster in the presence of anthropogenic noise $(\bar{x} \pm S E=$ $55.89 \pm 1.08$ days) than without it $(\bar{x} \pm S E=60.45 \pm 1.13$ days) (Figure 4.5). Neither male body size (PC1 size) nor residual mass at adulthood were significantly affected by the acoustic social environment's signalling density treatment, the anthropogenic noise treatment, or their interaction (Table 4.6). 
Table 4.6: Results of the linear models examining effects of acoustic treatment and season on development time, adult body size (PC1

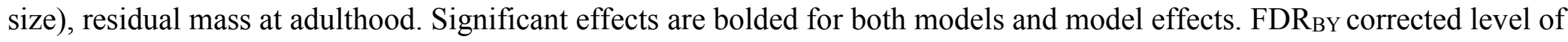
significance is $P=0.024(\mathrm{~N}=350)$. Parameter effects are included in Appendix 2, Table A2.5.

\begin{tabular}{|c|c|c|c|c|c|c|c|}
\hline Model & $\mathbf{R}^{2} \mathbf{a d j}$ & $\mathbf{F}_{\text {model }}$ & $\mathbf{P}_{\text {model }}$ & Model Effect & DF & $\mathbf{F}$ & $\mathbf{P}$ \\
\hline \multirow[t]{4}{*}{ Hatching to Wing pad } & 0.09 & 5.10 & $<0.0001$ & Season & 3 & 4.95 & 1.70 \\
\hline & & & & Signalling Density & 2 & 15.07 & $<0.0001$ \\
\hline & & & & Anthropogenic Noise & 1 & 0.81 & 0.37 \\
\hline & & & & Signalling Density*Anthropogenic Noise & 2 & 2.95 & 0.05 \\
\hline \multirow[t]{4}{*}{ Wing pad to Adult Eclosion } & 0.07 & 4.05 & $<0.0001$ & Season & 3 & 6.68 & $<0.0001$ \\
\hline & & & & Signalling Density & 2 & 3.92 & 0.021 \\
\hline & & & & Anthropogenic Noise & 1 & 0.50 & 0.48 \\
\hline & & & & Signalling Density*Anthropogenic Noise & 2 & 2.82 & 0.06 \\
\hline \multirow[t]{4}{*}{ Hatching to Adult Eclosion } & 0.13 & 7.40 & $<0.0001$ & Season & 3 & 5.21 & 0.0016 \\
\hline & & & & Signalling Density & 2 & 17.43 & $<0.0001$ \\
\hline & & & & Anthropogenic Noise & 1 & 1.22 & 0.27 \\
\hline & & & & $\begin{array}{l}\text { Signalling Density*Anthropogenic } \\
\text { Noise }\end{array}$ & 2 & 4.82 & 0.0086 \\
\hline \multirow[t]{4}{*}{ PC1 (Size) } & 0.03 & 2.50 & 0.0121 & Season & 3 & 5.49 & 0.0011 \\
\hline & & & & Signalling Density & 2 & 0.75 & 0.47 \\
\hline & & & & Anthropogenic Noise & 1 & 0.21 & 0.65 \\
\hline & & & & Signalling Density*Anthropogenic Noise & 2 & 0.27 & 0.77 \\
\hline \multirow[t]{4}{*}{ Residual Mass } & 0.05 & 3.38 & 0.0009 & Season & 3 & 3.48 & 0.0163 \\
\hline & & & & Signalling Density & 2 & 3.12 & 0.05 \\
\hline & & & & Anthropogenic Noise & 1 & 2.36 & 0.13 \\
\hline & & & & Signalling Density*Anthropogenic Noise & 2 & 2.93 & 0.05 \\
\hline
\end{tabular}




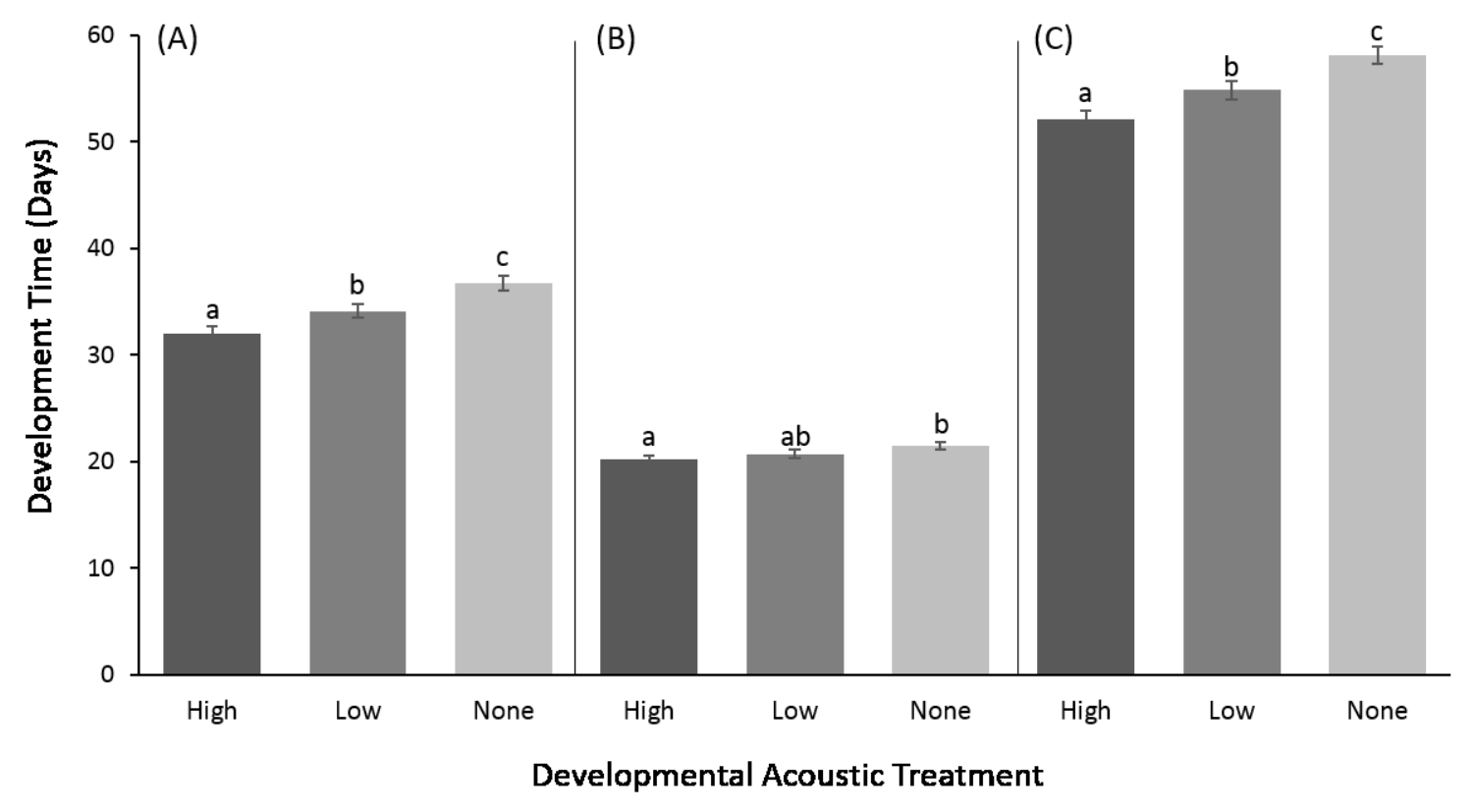

Figure 4.4. Effect of developmental acoustic treatment (signalling density) on development time (days) for the different life stages: (A) Hatching to Wing pad, (B) Wing pad to adult eclosion, and (C) Total Development Time. Error bars are standard errors and lowercase letters denote significant differences between acoustic treatment groups for each life stage. 


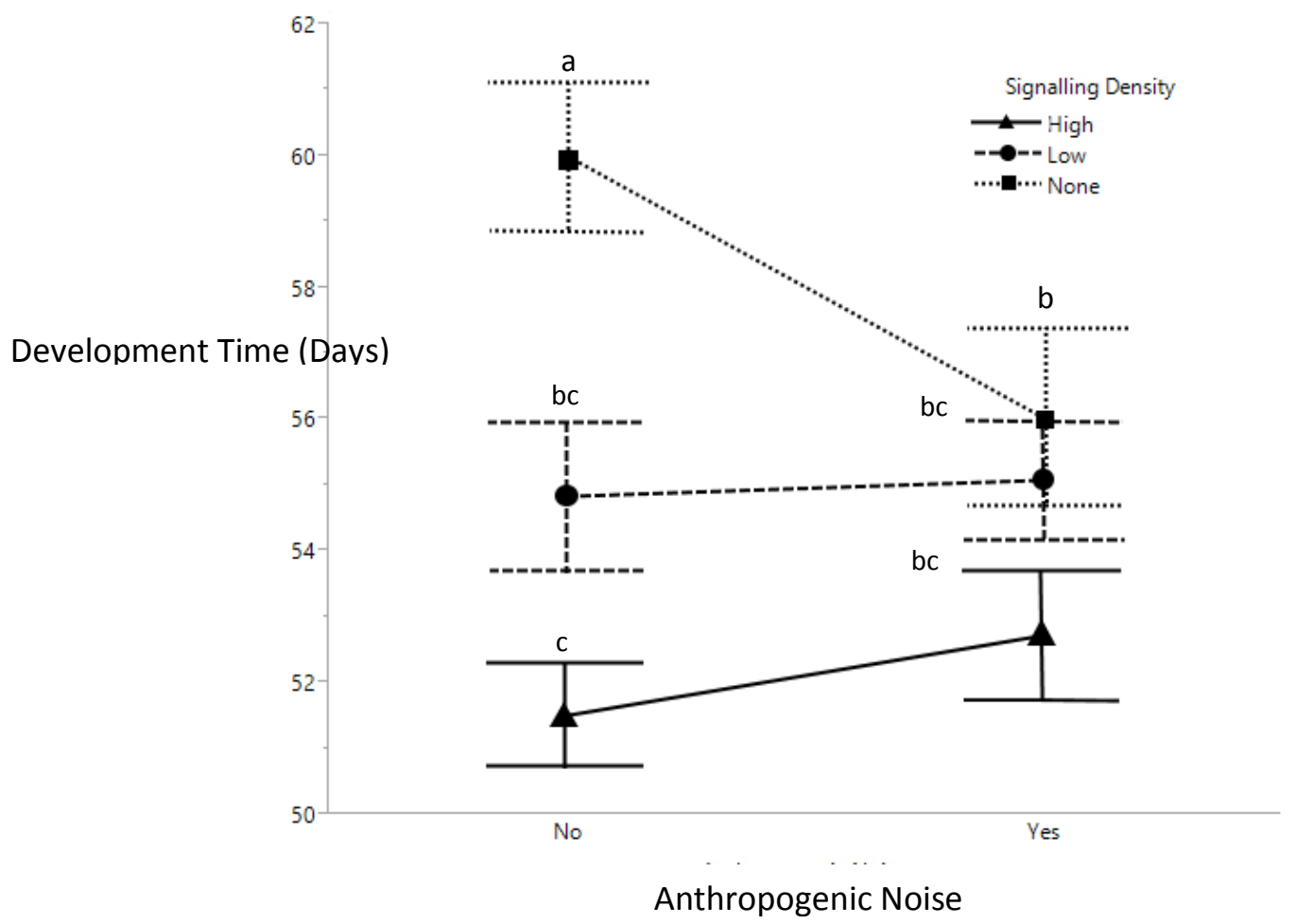

Figure 4.5. The interaction of signalling density and anthropogenic noise as it affects total development time (Days from Hatching to Adult eclosion). Bars are standard errors and letters denote significant differences. 


\subsubsection{Impact of Development Time on Adult Body Size}

Development time influenced body size at adult eclosion, but this effect was only significant in the later part of development (from wing pad to adult eclosion).

Specifically, males that were faster to develop were larger at adult eclosion than males that developed slowly $(P<0.0001$; Table 4.7). Development time did not have any effect on residual mass at adult eclosion (Table 4.7). 
Table 4.7. Results of the linear models examining effects of development time on body size (PC1 size) and residual mass at adulthood in males $(\mathrm{N}=350)$. Significant effects are bolded for both models and model effects, FDR $\mathrm{BY}_{\mathrm{B}}$ corrected $P=0.027$. Parameter effects are included in Appendix 2, Table A2.6.

\begin{tabular}{lrrrlrrr}
\hline Model & $\mathbf{R}^{2}$ adj & $\mathbf{F}_{\text {model }}$ & $\mathbf{P}_{\text {model }}$ & Model Effect & DF & $\mathbf{F}$ & $\mathbf{P}$ \\
\hline PC1 size & $\mathbf{0 . 0 9}$ & $\mathbf{8 . 2 9}$ & $<\mathbf{0 . 0 0 0 1}$ & Season & $\mathbf{3}$ & $\mathbf{3 . 5 8}$ & $\mathbf{0 . 0 1 4 1}$ \\
& & & & Days from Hatching to Wing pad & 1 & 0.04 & 0.84 \\
& & & & Days from Wing pad to Adult Eclosion & $\mathbf{1}$ & $\mathbf{2 2 . 1 7}$ & $<\mathbf{0 . 0 0 0 1}$ \\
Residual Mass & $\mathbf{0 . 0 2}$ & $\mathbf{2 . 7 4}$ & $\mathbf{0 . 0 1 9 2}$ & Season & $\mathbf{3}$ & $\mathbf{3 . 5 1}$ & $\mathbf{0 . 0 1 5 5}$ \\
& & & & Days from Hatching to Wing pad & 1 & 0.01 & 0.94 \\
& & & & Days from Wing pad to Adult Eclosion & 1 & 1.78 & 0.18 \\
\hline
\end{tabular}




\subsection{Discussion}

\subsubsection{Overall Aggression}

The focal males (raised in isolation and exposed to one of the acoustic social environment soundtracks) won more fights than expected by chance alone compared to their opponents (raised in a communal setting). Further, focal males were more aggressive than their opponent males. The focal males were raised in individual containers in complete physical isolation, with little to no visual, chemical, or tactile information from other individuals. This differed from the opponent males, as they were reared in groups, with extensive visual, chemical and tactile stimulation between individuals. Given that developmental treatment did not influence the level of aggression, it appears that being reared in physical isolation is the key variable that affected the outcome of these aggressive contests.

The finding that focal males are more aggressive and win more fights than opponent males suggests that there may be a difference between the effects of the acoustic social experience and the physical social experience, as each conveys different specific information. While acoustic social experience might indicate levels of future male density and possibly male quality, juveniles cannot use information contained in the acoustic environment to provide information about levels of future female density or female quality. Conversely, the physical social experience may convey details about future operational sex ratios and female and male quality through visual, chemical, vibratory, tactile and acoustic cues (Kasumovic \& Brooks 2011). Given this, lab reared opponent males were likely exposed to more social information than the focal males, as the opponent males were exposed to both male and female juveniles until their wing pad 
eclosion, and then to other juvenile males until their adult eclosion. This increased early social exposure during development appears to have been enough to decrease their adult aggressive behaviour. This effect also occurs in vertebrates and invertebrates alike, where the cost of juvenile isolation is not only increased aggression but also reduced foraging and exploration, reduced general social interaction, and decreased mating success (Lihoreau et al. 2009; Hol et al. 1999; Miller et al. 2012).

\subsubsection{Focal Male Aggression}

When I just examined my focal males, I found that larger focal males were more aggressive and more likely to win fights than smaller focal males. Adult male body size is an important indicator of male fitness and is often considered an honest trait of male condition (Whitman 2008; Sokolovska et al. 2000). My findings align with many other studies that show that larger male field crickets are more likely to win aggressive contests (Reaney et al. 2011). Winning a fight has important fitness consequences, as winners or dominant males are often preferred by females (Rantala \& Kortet 2004; Kortet \& Hedrick 2005).

While the developmental acoustic environment did not directly affect aggression levels, it may have had an indirect effect through how development time influences body size (see Appendix 3 for discussion; Figure 4.6). Specifically, males raised in the High density environment developed faster than males in either the Low density or None environment, and males that developed faster were larger at adulthood. These indirect effects highlight the complexity of only studying behaviour at a single point in an animal's life, without either controlling or at least having knowledge of important earlier life history events, as phenotypic outcomes may result from both direct and indirect 
selection pressures (French \& Cade 1989; Sih et al. 2004). 


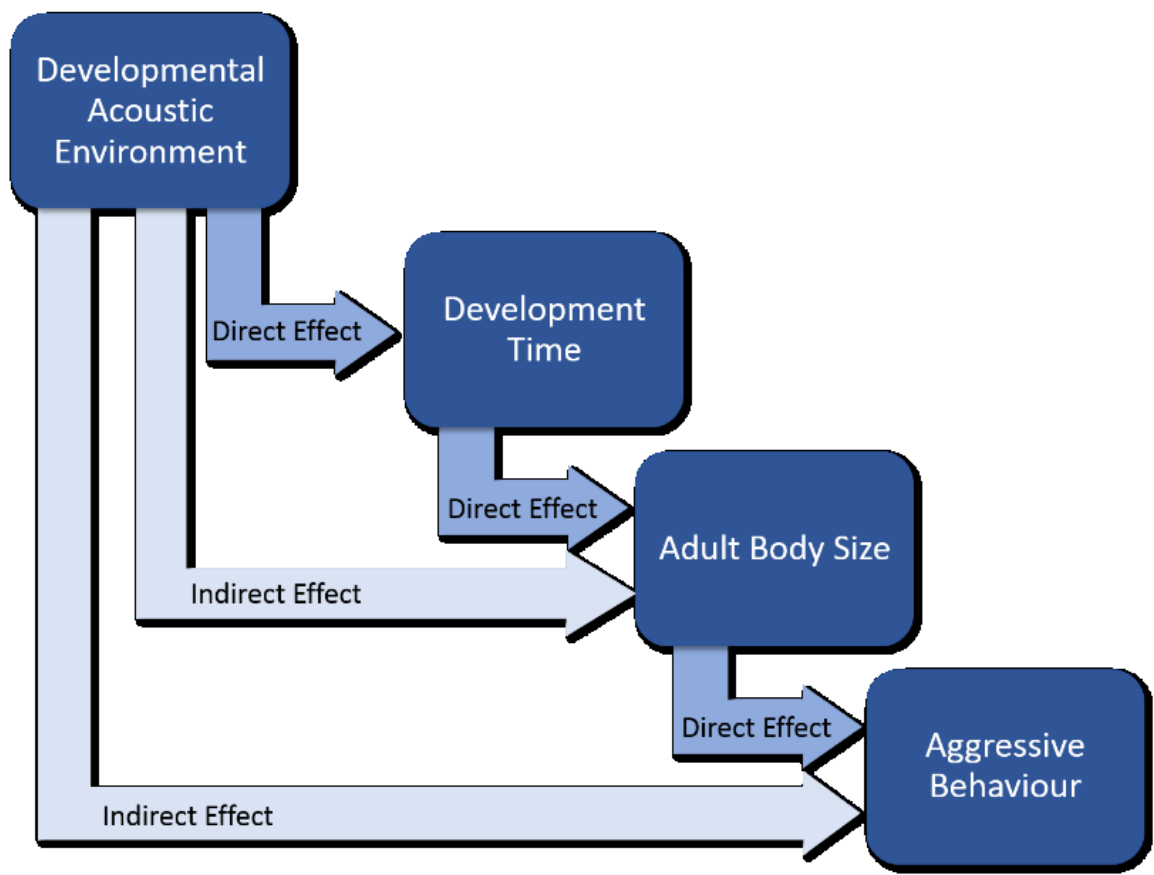

Figure 4.6. Schematic highlighting the indirect effects that rearing acoustic environment can have on adult behaviours through steps of smaller, direct effects on different aspects of life history traits. 


\subsubsection{Winners versus Losers}

Because winners and losers often display different behaviours (Rutte et al. 2006), I also examined how developmental acoustic social environment treatment influenced aggression in winners and losers separately. Winner aggressive behaviour was not significantly affected by differences in the developmental acoustic treatment. Perhaps this is not surprising, as winners were already more aggressive than losers; any increase in aggression is unlikely to confer a fitness advantage and instead is more likely to incur a fitness cost. Given aggressive fights are energetically and physically demanding (Hack 1997), the cost of increasing levels of aggression beyond achieving dominance will be high and therefore are unlikely to outweigh possible benefits. For example, in male field crickets, winners only increase aggression in the presence of a female audience and not in the presence of a male audience (Fitzsimmons \& Bertram 2013; Montroy et al. 2016). This increase in aggressive behaviour when a female audience is present may act as an advertisement to potential mates, thus gaining potential mating opportunities and offsetting the risks of increased aggression. Aggression contests in nature should thus reflect a sum of practical and performative aggressive behaviours. In the present study, dominant males may have lacked incentive to continue increasing aggressive behaviour beyond practical levels, as audiences were not present.

In subordinate males, (losers of the aggressive contests), aggressive behaviour was significantly affected by differences in focal male acoustic social environment. Losing males that were reared in social isolation (the None environment) were more aggressive than losing males reared in either the High or Low acoustic social environments. This finding is similar to other cricket studies that showed that social isolation during 
development results in more aggressive and dominant males (DiRienzo et al. 2012; Nagamoto et al. 2005). A lack of social experience leads to increased levels of aggression, perhaps mitigated by a reduction in the risks associated with contests when living in a low density social environment or amplified levels of stress. Increased aggression as the result of social isolation has been observed across several taxa, in addition to changes in other behavioural patterns (as discussed above). Interestingly, Stevenson and Rillich (2013) suggested that the heightened levels of aggression in socially isolated Mediterranean field crickets (G. bimaculatus) should be considered as the default state. They suggested that decreased aggression in socialized crickets may result from "social subjugation," with isolation allowing for recovery and return to the more aggressive state. In nature, male territories are often meters apart and adult malemale encounters are infrequent (Alexander 1961), thus resulting in a naturally more isolated environment and theoretically higher "default" levels of aggression. In partial support of this, Fitzsimmons and Bertram (2013) found that wild-caught G. veletis males were significantly more aggressive and had higher levels of victory displays than their first generation laboratory-reared descendants. Fitzsimmons and Bertram's (2013) finding was, however, audience-dependent and suggested that wild-caught males were more responsive to their social environment. Natural habitats for field crickets are dynamic, as male mate attraction signals are overheard between territories, females travel between mates, and juveniles are likely to encounter other juveniles and adults before establishing their own territory (Alexander 1968; Alexander 1961). In my study, subordinate males raised in total isolation could therefore have experienced a heightened level of natural aggression whereas opponent males raised with physical and acoustic social experience 
are undergoing some level of social subjugation as suggested by Stevenson \& Rillich (2013). One reason this effect was only observed in subordinate, and not dominant, males could relate to the already heightened levels of aggression in all my focal crickets. The levels of aggression in the winning males may have already been too high for the subtle effects of acoustic isolation to be observed.

High levels of aggression in winning males may also explain why I saw no effects of anthropogenic noise on aggression levels - physical isolation during development alone is possibly enough to result in heightened aggressive levels in this species. While the effects of anthropogenic noise on animal aggressive behaviour have been relatively unexplored, emerging studies suggest that noise pollution may lead to increased aggression levels. For example, in the white-crowned sparrow (Zonotrichia leucophrys) as territory noise increases males approach a stimulus more closely and for longer periods of time (indicative of higher aggression levels) (Phillips \& Derryberry 2018). Long-term effects of anthropogenic noise on animal aggression are still unknown and future studies should aim to explore their relationship.

Overall, my study highlights the importance and complexity of examining adult aggressive behaviours across an individual's life. Future research should focus on specific differences between acoustic social isolation and other forms of social deprivation to help elucidate the relationships between aggression and social experience. 


\section{Chapter 5: Field cricket signalling behaviour is not influenced by the developmental acoustic social environment or anthropogenic noise}

\subsection{Abstract}

Male sexual advertisement signals convey important information about their current physical condition and quality as a potential mate to nearby females. Acoustic signals exist in a social network and so also provide information to conspecific males concerning rivalry, aggressiveness, and territory holdings. Additionally, unintended information can be shared in this network such as general population densities and overall population quality. Eavesdroppers thus have access to acoustic information with little to no effort. When male mate attraction signals are overheard by unintended juvenile recipients, the juveniles can use the information contained in the signals to estimate the make-up of their future social environment and then alter their life-history trajectories accordingly. Juveniles that hear many acoustic signals may expect to face heightened competition as adults. They may therefore invest heavily in energy stores and have higher quality signals at adulthood than juveniles that hear no acoustic signals and as a result expect to face little to no future competition. Furthermore, acoustic signals may be interrupted or obfuscated by anthropogenic noise pollution, a real concern with rapid urban expansion. If this occurs during development, noise pollution has the potential to disrupt incoming signals so that eavesdropping juveniles are unable to accurately assess future population dynamics. To measure the effect of the acoustic environment, I examined changes in adult male signalling behaviour in response to mate attraction signalling (the acoustic social environment) and noise pollution experienced during development. I reared male fall field crickets while exposing them to one of six different acoustic social 
environments that differed in the density of signalling adult males (High, Low, and None) and anthropogenic noise (Present or Absent). Contrary to my predictions, juvenile acoustic social environment did not affect adult male signalling behvaiour. There were no differences in any fine scale signal parameters, time spent signalling, or amplitude between the High, Low, and None acoustic social environments. There was, however, an indirect effect: males that experienced acoustic signalling during development (High and Low) developed faster than males reared in acoustic isolation (None), and faster development times resulted in larger adult body sizes. This indirect effect is important because larger males produced more energetically costly high amplitude signals than smaller males. Additionally, there was a near significant interaction between juvenile acoustic social density and anthropogenic noise. Males raised in acoustic isolation (None) signalled at higher amplitudes when reared with anthropogenic noise than those reared without it. These results highlight the importance of recognizing the indirect effects of developmental experience while also providing early implications of anthropogenic noise effects in Orthoptera.

\subsection{Introduction}

Signal dynamics include the transfer of information via one individual (the signaller) to one or more individuals (the receiver) and have evolved to influence the immediate or future behaviour of the receiver(s) (Wiley 1983; Endler 1993). Signals can be produced using visual, vibratory, chemical, auditory, electrical, tactile, and thermal mechanisms (Partan \& Marler 2005). For example, visual signals include the colourful plumage of birds (McGraw 2008) and pigmentation in fish (Price et al. 2008), as well as 
ornamentations such as horns in ungulates (Bergeron et al. 2010) or beetles (Emlen et al. 2012). Several species of spiders (Sivalinghem et al. 2010; Gordon \& Uetz 2011) and frogs (Narins 1990; Rodríguez et al. 2004) use vibrations to send signals to receivers through the substrate. Chemical signals, or pheromones, are transferred over short distances or via direct proximity in both land vertebrates and invertebrates (Partan \& Marler 2005) and across longer distances in marine environments (Atema 1995).

Additionally, acoustic or auditory signals are widespread among both vertebrates and insects (Pollack 2017). Acoustic signals can be created using multiple methods including the movement of air through organs (birds, amphibians, and mammals), stridulation (insects and fish), and drumming or vibrations (fish, reptiles), (Radford et al. 2014). Furthermore, signals can be complex, relying on a combination of the aforementioned mechanisms. For example, wolf spiders (Schizocosa ocreata) use both visual and vibratory signals to attract females (Gordon \& Uetz 2011), which allows for effective communication across different substrates and for communicating with females when they are out of the line of sight.

The type of information that signals transmit can vary from individual (condition or personality), to social (species identification, aggression and dominance, warning, etc) (Greenfield 1997). Considerable research in behavioural ecology has been dedicated to investigating the kinds of information males communicate to females within their sexual signals. Male sexual signals often provide information about species identification and can also provide information about the males' current and/or past condition (Snijders \& Naguib 2017; Darwin 1871; Whattam \& Bertram 2011). Fisher (1930) suggested that a female's preference for conspicuous male mate attraction signals might increase her 
chances of mating with a male that is in better condition.

Signals that exhibit condition-dependent expression should be honest (closed to cheaters) because they are usually costly to produce and maintain as they are transmitted using complex behavioural and physiological processes (Andersson 1994; Burk, 1988; Laidre \& Johnstone, 1872). As such, males in poor condition should not be able to maximize signal conspicuousness, whereas males in good condition should be better able to bear these costs (Hunt et al. 2004; Rowe \& Houle 1996; Kotiaho et al. 2001; Tomkins et al. 2004). Honest signals should also be selected for because they increase the fitness of the receiver(s), and signalling systems are shaped by the mutual interests of both signallers and receivers (Johnstone 1995; Guilford \& Dawkins 1991).

In nature, signalling often occurs within a network, where unintended receivers (audiences) can detect the signal (eavesdroppers; (Tomkins et al. 2004; Peake et al. 2005). Audience members who eavesdrop on signals intended for others can use this information that is contained in their 'social environment' to alter their own behaviour or life-history trajectories (Kasumovic \& Brooks 2011). For example, male spiders (Argiope bruennich) exposed to female pheromones during development have shortened subadult developmental stages compared with males not exposed to the female cues (Cory \& Schneider 2017). Female field crickets (Teleogryllus oceanicus) exposed to male acoustic signal playbacks during development were more discriminating and less responsive to male signals as adults compared to females who developed in a silent acoustic environment that lacked social information input (Bailey \& Zuk 2008). Early social isolation effects can also be found in veiled chameleons, (Chamaeleo calyptratus), where lizards reared in isolation were more submissive, were poorer foragers, and adopted 
darker and duller colours (Ballen et al. 2014). Audiences can in turn influence the signaller's behaviour, as seen in the Jamaican field cricket, (Gryllus assimilis), where males are more likely to initiate and escalate fights in the presence of a female audience compared to a lack of audience (Montroy et al. 2016). Given this, an individual's current social environment has the potential to provide a template for how social and demographic factors may influence their future (Moore et al. 1997; Danchin et al. 2004; Perrin et al. 2012).

Here I investigated how the acoustic social environment experienced by juvenile male fall field crickets, G. pennsylvanicus, affected their adult mate attraction behaviours. Male field crickets produce audible sexual signals in the form of long distance mate attraction signals that function to attract potential mates. Female field crickets often prefer larger males that produce the most conspicuous acoustic mate attraction signals (Hedrick 1986; Simmons 1988; Tolle \& Wagner 2010). Female field cricket receivers can use components of the male's acoustic long distance mate attraction signal to ascertain the benefits of mating with him, as acoustic signals often convey information about the male. For example, long distance mate attraction signals often convey information about the male's current nutritional condition (Scheuber et al. 2003a; Whattam \& Bertram 2011), his past nutritional condition (Whattam \& Bertram 2011), his body size (Harrison et al. 2013; Bertram \& Rook 2012), and his age (Judge 2011; Fitzsimmons \& Bertram 2013). Conspicuous long distance mate attraction signals can also simultaneously alert competing males of their presence, either as intentional receivers or as unintended eavesdroppers. Competitors can use components of the male's mate attraction signal to ascertain the benefits of fighting him for his territory. 
Juveniles that overhear conspecific's mate attraction signals have the potential to use these acoustic social environmental cues as indicators about the demographics and quality of males in their immediate environment and possible future population. Juveniles could therefore alter their own developmental trajectories to enable them to be better competitors in the future environment (Kasumovic et al. 2011). As sexual signals can be energetically costly to produce, resource allocation during development towards growth and signal production could produce a fitness benefit for the listening male by insuring that he is big enough and in good enough condition to signal conspicuously upon maturity. Males that produce more conspicuous signals are more likely to mate with females and outcompete rivals when competition is high (Cade \& Cade 1992; Judge et al. 2008).

Research to date suggests that the expectant social environment does in fact affect male field cricket development and behaviours upon maturity. For example, male field crickets (Teleogryllus oceanicus) exposed to an acoustic environment representative of high density signalling during development exhibited less satellite behaviour, invested more in reproductive tissue, and were in better condition at adulthood than males exposed to a silent acoustic environment during development (Bailey et al. 2010). Further, Kasumovic et al. (2011) studied male plastic response in Teleogryllus commodus and found that males matured more rapidly, at a smaller size, and signaled for mates more often when reared in a low density acoustic signalling environment. These studies suggest that juvenile crickets that experience acoustic mate attraction signals during development obtain an informational template about the social environment that they will experience in the future, when they are adulthoods. Given this, the impact of juveniles 
using acoustic cues to inform them about their potential future social environment in adulthood should be considered when studying behavioural plasticity.

I therefore predicted that males reared in the isolation and low density acoustic environment should perceive lower adult competition and should signal more often and produce louder, more conspicuous signals than males raised in a high density acoustic environment. I made these predictions because when the number of same-sex individuals in a population is low, there is a decrease in intersexual competition; in adult crickets, lower male population densities results in an increase in male signalling effort (Cade \& Cade 1992).

Acoustic male advertisement signals also have the potential to be affected by anthropogenic noise pollution. An increase in urban noise has been shown to affect acoustic signalling in several species. Changes to signals can occur through shifts in signal timing, redundancy, amplitude, and frequency (Symes \& Price 2015). For example, male European robins (Erithacus rubecula) from noisy territories sing less complex and shorter songs with higher minimum frequencies, at a lower rate compared with males from quiet territories (Montague, Danek-Gontard, and Kunc 2012). An increase in both time spent signalling and amplitude is found in the suburban serin (Serinus serinus) when urban noise increases, a metabolic costly behaviour (Diaz et al. 2011). There is also emerging evidence that anthropogenic noise can alter male acoustic signalling in tree crickets (Oecanthus pellucens, Orci, Petr, and Barta 2016), and grasshoppers (Chorthippus biguttulus, Lampe et al. 2012), and also interfere with female phonotaxis towards said signals (Gurule-Small \& Tinghitella 2018; Schmidt et al. 2014; Costello \& Symes 2014). Therefore, I also established if and how the presence of 
anthropogenic noise, specifically urban noise pollution, interfered with the juveniles' ability to eavesdrop on the acoustic cues, resulting in different signalling behaviours upon maturity. I predicted that anthropogenic noise pollution should mask the acoustic signals, making it difficult for the juveniles to accurately predict the composition of their future adult population. Anthropogenic noise should therefore result in juvenile males expecting less male-male competition, and as a result, signal for mates more often and louder as adults, regardless of the acoustic social environment that they were reared in.

\subsection{Methods}

\subsubsection{Study Species and Cricket Rearing}

To test how the developmental social environment affects adult signalling behaviour I used laboratory-reared North American Fall Field crickets, $G$. pennsylvanicus. I randomly assigned each one-week old cricket (housed in individual containers) to one of 6 acoustic environment treatments, and then placed each individual container into a larger communal treatment bin. The communal treatment bins were lined with acoustic foam to prevent sound contamination across treatments. Each communal treatment bin lid contained 4 speakers for playback of acoustic environment soundtracks. I used 6 different acoustic soundtrack treatments in a $3 \times 2$ factorial design: 3 with different densities of male acoustic signals, and 3 with these male acoustic signals overlaid with anthropogenic noise. Crickets were kept on a 14h:10h light:dark photoperiod at $28 \pm 2{ }^{\circ} \mathrm{C}$. Thrice weekly, each juvenile cricket had its food and water replenished and was checked for wing pad eclosion or adult eclosion. Chapter 2 provides a detailed description of how I produced my six different acoustic treatments, how I housed my individual crickets during rearing in their different acoustic treatments, how I 
quantified body size, and the other general protocols that I used for all data chapters.

Once crickets reached adult eclosion I weighed them and then returned them to the acoustic treatment they developed in, with the exception that I housed these adult males in separate communal treatment bins away from the juveniles. I separated adults from developing juveniles to minimize acoustic interference within the juvenile acoustic environment, because adult male G. pennsylvanicus can produce acoustic signals as early as 3-4 days post-imaginal moult (Cade \& Wyatt 1984; Judge 2011). I housed a maximum of 6 adult males together at any one time to ensure that any acoustic signals produced by the experimental males were kept to a minimum to prevent interference with their assigned acoustic social environment. Experimental crickets therefore experienced their assigned acoustic environment treatment from 1 week post hatch until they had their acoustic mate attraction signals monitored at 7 days post-imaginal moult. At 7 days postimaginal moult I ran each male through either aggression and signalling trials, or just signalling trials. Information quantifying adult signalling behaviour lies below; for information on the aggression trials, please refer to Chapter 4 .

\subsubsection{Adult Male Signalling Behaviour}

Male acoustic signalling behaviour was continuously monitored from day 7 to 14 postimaginal moult (7 days total) using the Electronic Acoustic Recording System (EARS-II; designed and developed by Cambridge Electronic Design, Cambridge, UK). The EARS-II system is composed of 96 Styrofoam containers individually lined with acoustic foam to avoid sound contamination. Each container has an LED light and microphone set into the lid which is monitored by Cricket software that filters out background noise and auto-adjusts its amplitude threshold for quiet or loud individuals 
while simultaneously monitoring and recording individual mate attraction signals (Whattam \& Bertram 2011; Bertram et al. 2004). Using the EARS II system, I recorded time spent signalling (min/24 h period) and 9 fine scale signalling parameters (Figure 5.1), including: pulse duration (ms), interpulse duration (ms), pulse rate (P/min), pulses per chirp, chirp duration (ms), interchirp duration (ms), chirp rate ( $\mathrm{Ch} / \mathrm{min})$, signal amplitude (Pa; converted to decibels using $20 \mathrm{X} \log ($ mean amp $(\mathrm{Pa}) / 0.00002 \mathrm{~dB})$ ), and carrier frequency $(\mathrm{Hz})$. Acoustic files were analyzed to produce a summary of mean signalling parameters using Spike2 v6.12 (Cambridge Electronic Design, Cambridge, UK). Because the EARS II system quantifies individual acoustic signalling behaviour, I could not continue to expose each cricket to their assigned acoustic environment treatment as the broadcasted chirps would be detected by the EARS II. Therefore, each individual was held in silence during the quantification of their signalling behaviour phase of the experiment. After their signals were recorded, males were removed from the EARS II containers, weighed and then euthanized via freezing. Male signalling behaviour was analyzed using adults that eclosed between May 6, 2015 to November 18, 2016. 


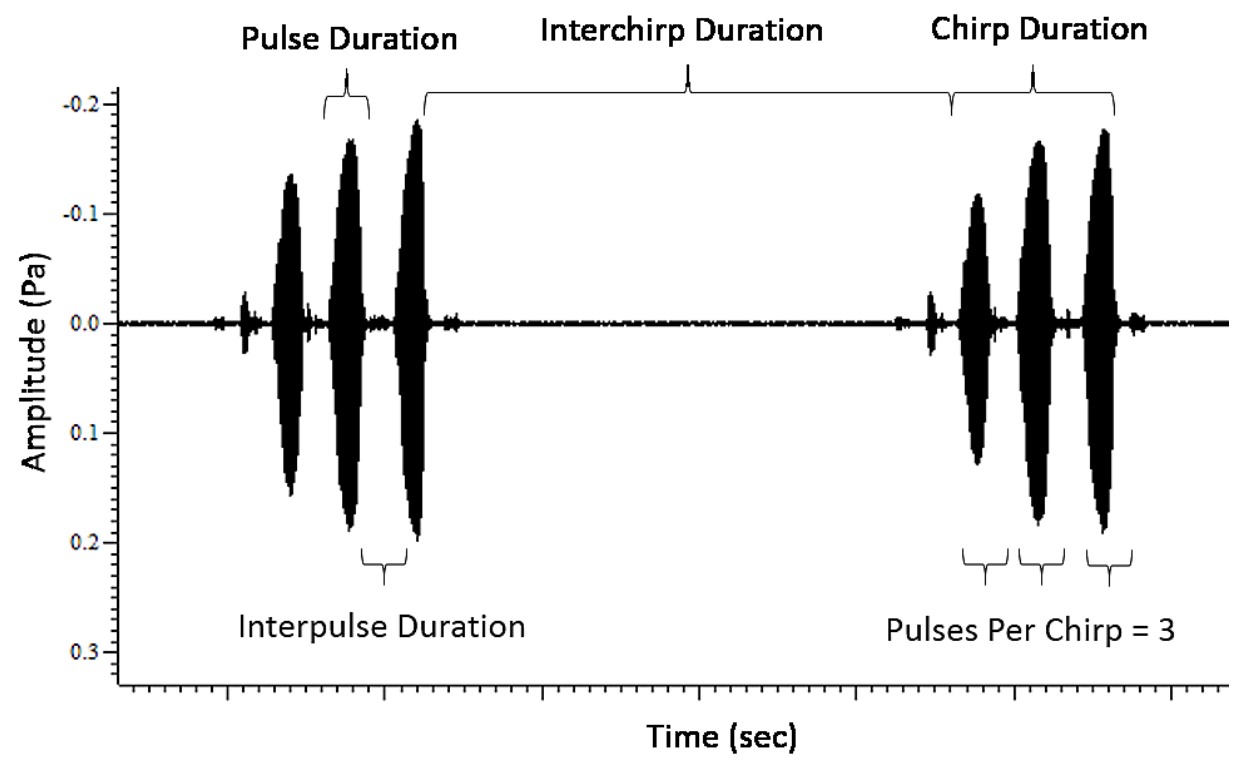

Figure 5.1. Waveform of 2 chirps from a G. pennsylvanicus long distance mate attraction signal exhibiting some of the parameters that are recorded and measured by the EARS II. 


\subsubsection{Statistics}

All statistical analyses were completed using JMP statistical software (version 11.0.0 SAS Institute). To determine adult residual mass and body size at adult eclosion, I combined body size measurements using a principle component analysis (PCA) and extracted orthogonal vectors from pronotum length, pronotum width, and head width. The first principal component (PC1 size) explained 93\% of the variation in male size (Eigenvalue $=2.8)$; all size measures loaded equally on PC1 size (eigenvectors ranged from 0.57 to 0.58 ). I then regressed body size (PC1 size) against body weight to determine residual mass at adult eclosion.

I performed a PCA to extract orthogonal vectors from eight of the fine-scale acoustic signalling characteristics (pulse duration, interpulse duration, pulse rate, pulses per chirp, chirp duration, interchirp duration, chirp rate, and carrier frequency). I excluded time spent signalling and amplitude from this PCA because I had made explicit predictions about how a male should signal more often and louder if he was reared in an environment with less adult signalling playback and I did not want these variables confounded by the other signalling parameters in the PCA. For interpretation of the PCA, I performed varimax rotation on the first 2 principle components. The first two rotated principle components were selected (signal factor 1 and 2) as they had eigen-values above 1 (Kaiser 1964) and satisfied the scree test for factor rotation conditions (Cattell 1966). Signal factor $1($ Eigenvalue $=4.31)$ explained $62 \%$ of the signalling variation and was indicative of longer pulse and chirp durations produced at lower carrier frequencies: high scoring males signalled at lower carrier frequencies and produced longer mean pulses with shorter interpulse durations, more pulses per chirp and longer chirp durations. 
Signal factor $2($ Eigenvalue $=1.41)$ explained $20 \%$ of the signalling variation and was indicative of signalling effort: high scoring males had shorter mean daily interchirp durations and produced more pulses and chirps per second (Table 5.1).

I used linear models (LMs) to examine the effects of the acoustic environment experienced during development on adult males' long-distance mate attraction signalling behaviour. The dependent variables I used were signal factor 1 , signal factor 2 , time spent signalling (mean daily), and amplitude (mean daily, dB). The independent variables I used were the social environment's signalling density (High, Low, None), anthropogenic noise (Yes, No) and the interaction between signalling density and anthropogenic noise. I also included adult body size (PC1 Size), adult residual mass, participation in an aggression trial (Yes, No), and season (Winter, Spring, Summer, Fall) as covariates. Aggression trial was included to control for the fact that a subset of the males experienced an aggression trial prior to having their acoustic mate attraction signals monitored (detailed in Chapter 4). Season of hatching was included as a block effect to control for possible environmental changes throughout the year. I tested LMs for violations of normality by examining the distribution of residual diagnostic plots (residuals vs fitted values and Q-Q plots); I also tested LMs for violations of homoscedasticity by looking for dependency between the residuals and the fitted values. Normality and homoscedasticity assumptions were not violated. P-values were corrected for using false discovery rate $\mathrm{FDR}_{\mathrm{B}-\mathrm{Y}}$ method (for all models $P=0.02$ )(Benjamini \& Yekutieli 2001). Non-significant model interactions were removed for model simplification. 
Table 5.1. Rotated PCA factor loading values for signal factor 1 and 2 of 8 mean daily fine scale signalling compoents after varimax roation. A negative sign (-) on the loading indicates that higher levels of that factor are negatively associated with that signalling parameter. Loadings above 0.600 are shown in bold.

\begin{tabular}{lrr}
\hline Fine Scale Signal Component & Signal factor 1 & Signal factor 2 \\
\hline Eigenvalue & 4.31 & 1.41 \\
Percent & 62.32 & 20.35 \\
Cumulative Percent & 62.32 & 82.66 \\
Pulse Duration (ms) & $\mathbf{0 . 8 8 4}$ & 0.213 \\
Interpulse Duration (ms) & $\mathbf{- 0 . 7 7 1}$ & -0.522 \\
Pulses Per Chirp & $\mathbf{0 . 8 8 0}$ & 0.302 \\
Chirp Duration (ms) & $\mathbf{0 . 8 5 0}$ & 0.033 \\
Interchirp Duration (ms) & -0.134 & $\mathbf{- 0 . 8 4 8}$ \\
Pulse Rate (\#Pulses/sec) & 0.424 & $\mathbf{0 . 6 4 0}$ \\
Chirp Rate (Chirps/min) & 0.041 & $\mathbf{0 . 8 7 1}$ \\
Carrier Frequency (Hz) & -0.590 & -0.059 \\
\hline
\end{tabular}




\subsection{Results}

I analyzed the signalling behaviour of 231 adult males. Of these, 12 males did not signal and 28 males were outliers so I removed them from the analysis, resulting in a final count of 191 (High density: Anthropogenic noise 30, No Anthropogenic noise 35; Low density: Anthropogenic noise 27, No Anthropogenic noise 33; Isolation: Anthropogenic Noise 36, No Anthropogenic noise 30).

Juvenile acoustic social environment did not significantly affect adult male signalling behaviour (Table 5.2). Specifically, time spent signalling, amplitude, signal factor 1 (indicative of longer pulses and chirps produced at lower carrier frequencies) and signal factor 2 (indicative of higher signalling effort as males signalled at higher pulse and chirp rates) were not significantly influenced by either the density of acoustic signals or the amount of anthropogenic noise they were exposed to during development. There was, however, near-significant interactions between rearing density and anthropogenic noise for males reared in the Low density environments. Specifically, males reared in the Low environment tended to spend more time signalling $(P=0.06)$ and tended to signal at higher amplitudes $(P=0.05)$ in the presence of anthropogenic noise than without it (Table 5.2; Figure $5.2 \& 5.3$ ).

Signal factor 1 and amplitude were significantly affected by adult male body size (PC1 size), while time spent signalling showed a near-significant trend $(P=0.04)$ (Table 5.2). Larger males had a higher signal factor 1 scores (more pulses per chirp with longer pulse durations and shorter interpulse durations as well as longer chirp durations) (Figure 5.4). Larger adult males also signalled at higher amplitudes (Figure 5.4) and spent more time signalling throughout the day (Figure 5.4). Participation in an aggression trial before 
being placed in the EARS II (Chapter 4) did not explain inter-male variation in subsequent signalling behaviour (Table 5.2). 
Table 5.2. Linear model effects examining how acoustic social environment treatment, body size, residual mass, participation in an aggression trial, and season influence time spent signalling, amplitude, and signal factors 1 and 2. Significant effects are bolded.

FDR $_{\mathrm{BY}}$ corrected level of significance is $P=0.02$. Parameter estimates are included in Appendix 4, Table A4.1.

\begin{tabular}{|c|c|c|c|c|c|c|c|}
\hline Model & $\mathbf{R}^{2}{ }_{\text {adj }}$ & $\mathbf{F}_{\text {model }}$ & $\mathbf{P}_{\text {model }}$ & Model Effect & DF & $\mathbf{F}$ & $\mathbf{P}$ \\
\hline \multirow[t]{7}{*}{ Signal Factor 1} & 0.21 & 5.47 & $<0.0001$ & Season & 3 & 0.97 & 0.41 \\
\hline & & & & Signalling Density & 2 & 1.61 & 0.20 \\
\hline & & & & Anthropogenic Noise & 1 & 0.67 & 0.42 \\
\hline & & & & Signalling Density*Anthropogenic Noise & 2 & 0.72 & 0.49 \\
\hline & & & & PC1 Size & 1 & 45.59 & $<\mathbf{0 . 0 0 0 1}$ \\
\hline & & & & Residual Mass & 1 & 1.26 & 0.26 \\
\hline & & & & Aggression Trail & 1 & 1.59 & 0.21 \\
\hline \multirow[t]{7}{*}{ Signal Factor 2} & -0.007 & 0.87 & 0.57 & Season & 3 & 0.93 & 0.43 \\
\hline & & & & Signalling Density & 2 & 0.33 & 0.72 \\
\hline & & & & Anthropogenic Noise & 1 & 0.19 & 0.66 \\
\hline & & & & Signalling Density*Anthropogenic Noise & 2 & 0.51 & 0.22 \\
\hline & & & & PC1 Size & 1 & 1.69 & 0.19 \\
\hline & & & & Residual Mass & 1 & 0.83 & 0.36 \\
\hline & & & & Aggression Trail & 1 & 1.41 & 0.24 \\
\hline \multirow[t]{7}{*}{ Time Spent Signalling } & 0.012 & 1.21 & 0.28 & Season & 3 & 0.17 & 0.92 \\
\hline & & & & Signalling Density & 1 & 0.23 & 0.80 \\
\hline & & & & Anthropogenic Noise & 1 & 0.56 & 0.45 \\
\hline & & & & Signalling Density*Anthropogenic Noise & 2 & 2.92 & 0.06 \\
\hline & & & & PC1 Size & 1 & 4.34 & 0.04 \\
\hline & & & & Residual Mass & 1 & 0.07 & 0.80 \\
\hline & & & & Aggression Trail & 1 & 1.68 & 0.20 \\
\hline
\end{tabular}




\begin{tabular}{|c|c|c|c|c|c|c|c|}
\hline \multirow[t]{7}{*}{ Amplitude } & 0.37 & 9.63 & $<0.0001$ & Season & 3 & 3.29 & 0.0220 \\
\hline & & & & Signalling Density & 2 & 1.26 & 0.29 \\
\hline & & & & Anthropogenic Noise & 1 & 1.47 & 0.23 \\
\hline & & & & Signalling Density*Anthropogenic Noise & 2 & 3.04 & 0.05 \\
\hline & & & & PC1 Size & 1 & 78.85 & $<0.0001$ \\
\hline & & & & Residual Mass & 1 & 1.88 & 0.17 \\
\hline & & & & Aggression Trail & 1 & 1.28 & 0.26 \\
\hline
\end{tabular}




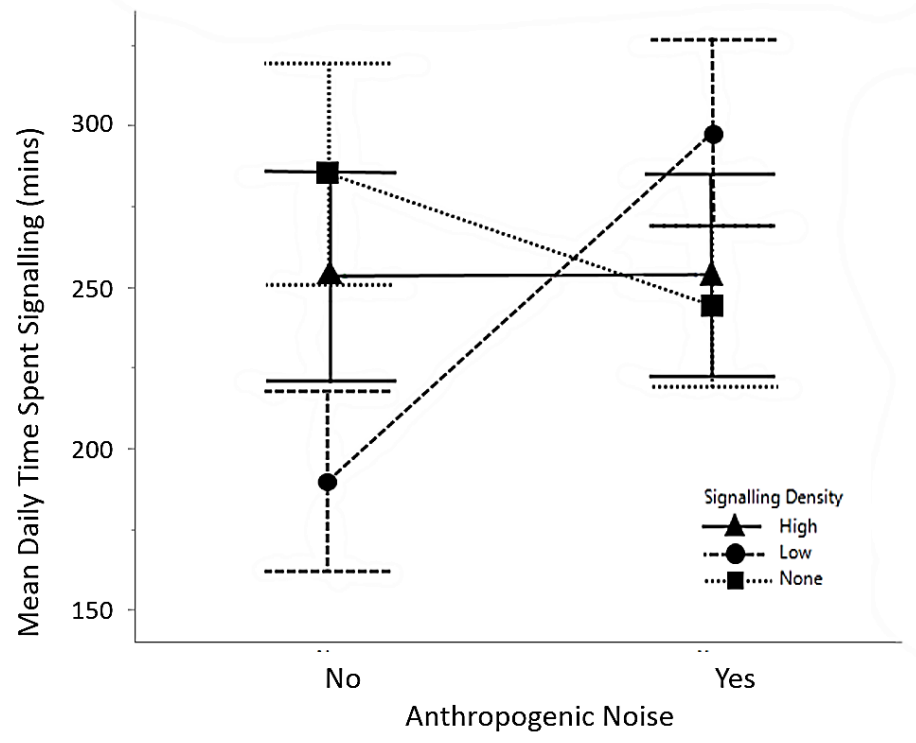

Figure 5.2. Effects of the interaction between anthropogenic noise and rearing environment density on male time spent signalling (mean daily mins) $(P=0.06)$. 


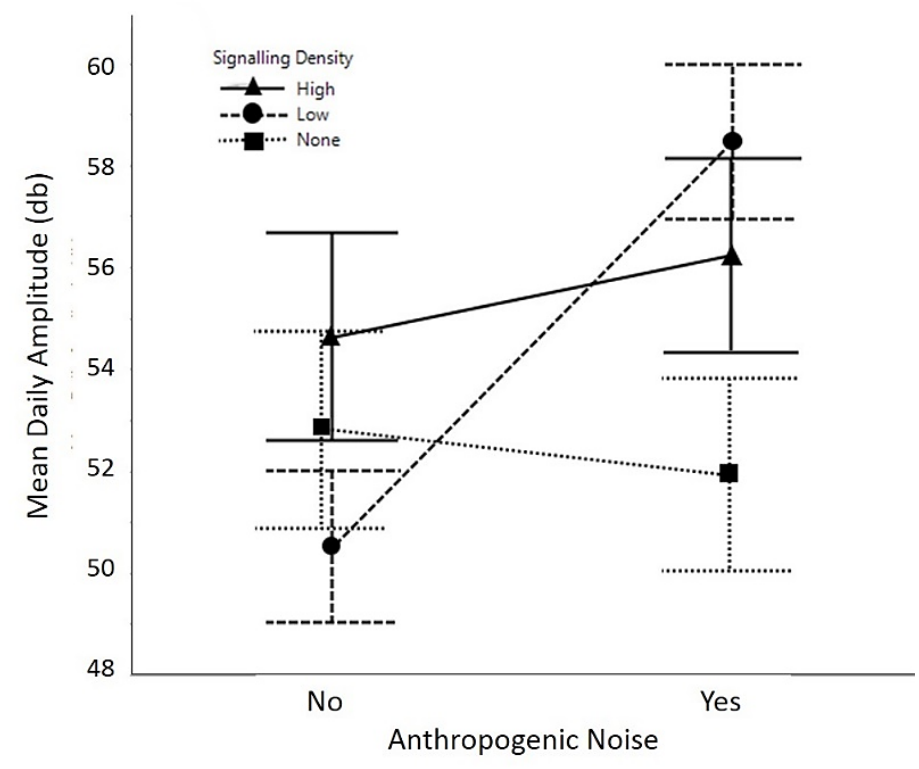

Figure 5.3. Effects of the interaction between anthropogenic noise and rearing environment density on male signalling amplitude (mean daily $\mathrm{dB})(P=0.05)$. 

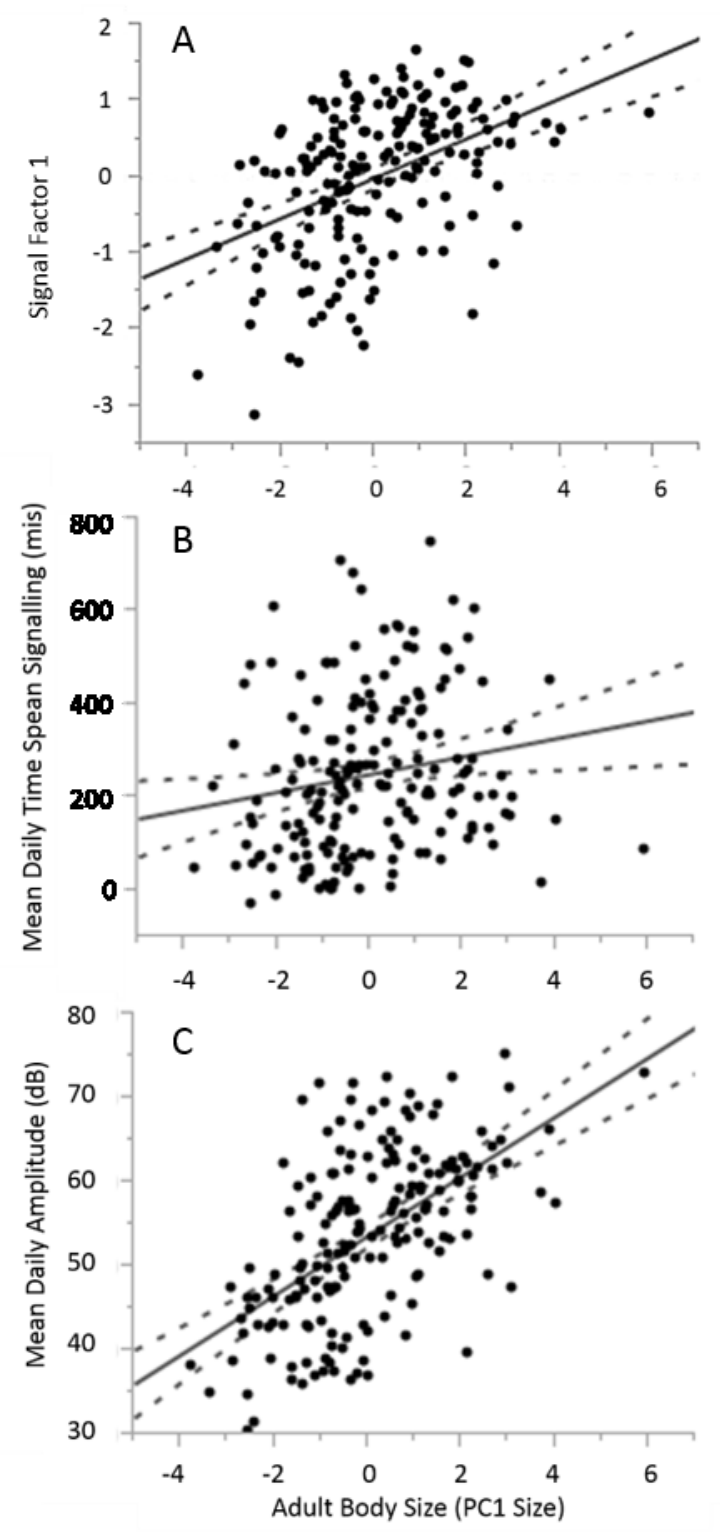

Figure 5.4. Leverage residual plots showing how adult body size (PC1) influence signalling components. Larger males signalled with (A) more pulses per chirp with longer pulse durations and shorter interpulse durations as well as longer chirp durations (signal factor 1), (B) signalled more throughout the day (non-significant trend), and (C) signalled at higher amplitudes (db). Solid line indicates the relationship between body size and signalling component after all other variables in the model has been accounted for; the dashed lines represent $95 \%$ confidence intervals. 


\subsection{Discussion}

My experiment was designed to test the hypothesis that the social environment experienced during development influences how male fall field crickets, Gryllus pennsylvanicus, signal as adults. Additionally, I wanted to test the hypothesis that anthropogenic noise confounds the ability of juveniles to accurately access future adult cohort levels, leading to a mismatch between their signalling behaviour and the social environment that they experienced as juveniles. My findings revealed no direct support for these hypotheses, as neither rearing density (High, Low, or None) nor the presence of anthropogenic noise significantly influenced adult male signalling behaviour. Research examining how the juvenile social environment effects adult behaviour in crickets is limited and sometimes contradictory. For example, in the Australian black field cricket (T. commodus) Kasumovic et al. (2011) found that males reared in high density environments with variable signal quality reduced signalling rate as adults. However, in a later study on the same species, they found that social environment during development had no direct effect on signalling rate, but that males exposed to high signalling densities as juveniles experienced a decline is signalling effort as they aged (Kasumovic, et al. 2012 a). Another layer of complexity is added when juvenile diet is also taken into consideration, as interactions between signalling treatment and diet differentially effected adult male signalling behaviour (Kasumovic, et al. 2012 b). The relationship between diet and signalling in field crickets is complex, with several studies highlighting the fact that juveniles and adults require different diets to optimize adult traits (Zajitschek et al. 2009; Reifer et al. 2018; Whattam \& Bertram 2011; Visanuvimol \& Bertram 2011). As all juveniles in my study received the same high quality diet, and the signal quality of the 
playback was not altered, perceived densities alone may not have been sufficient for males to alter their adult behvaiour.

I found that male body size significantly accounted for some of the variation in signalling behaviour, as larger males signalled with more pulses per chirp with longer pulse and chirp durations, at higher amplitudes. There was also a near significant trend for larger males to spend more time signalling. Acoustic communication imposes metabolic costs and animals with higher metabolic activity, with larger body sizes, and better access to resources often have advantages in signal production (Gillooly \& Ophir 2010). Long distance mate attraction signals in crickets are energetically expensive to produce (Hoback \& Wagner 1997; Wagner Jr \& Wyatt Hoback 1999) and males in better condition often produce more attractive signals (Judge et al. 2008; Harrison et al. 2013). Larger males tend to produce louder signals (Harrison et al. 2013; Bertram \& Rook 2012; Reifer et al. 2018) and signal for longer periods of time (Bertram \& Rook 2012). Here I confirmed that larger male G. pennsylvanicus produce energetically expensive signals. What is intriguing is that I have shown that the acoustic social environment influences development time, which in turn influences body size (Chapters 3,4). Juvenile males appear to perceive the acoustic cues present in the social environment, and as a result, alter their life history trajectory, developing faster than males reared in silence. Further, faster developers are larger. Thus, I hypothesize that the acoustic social environment in which juvenile males develop may indirectly influence male signalling behaviour via altering their development time, which in turn influences their body size (Figure 5.5). It is therefore important for future researchers to give consideration to the indirect effects of the acoustic social environment during rearing. 


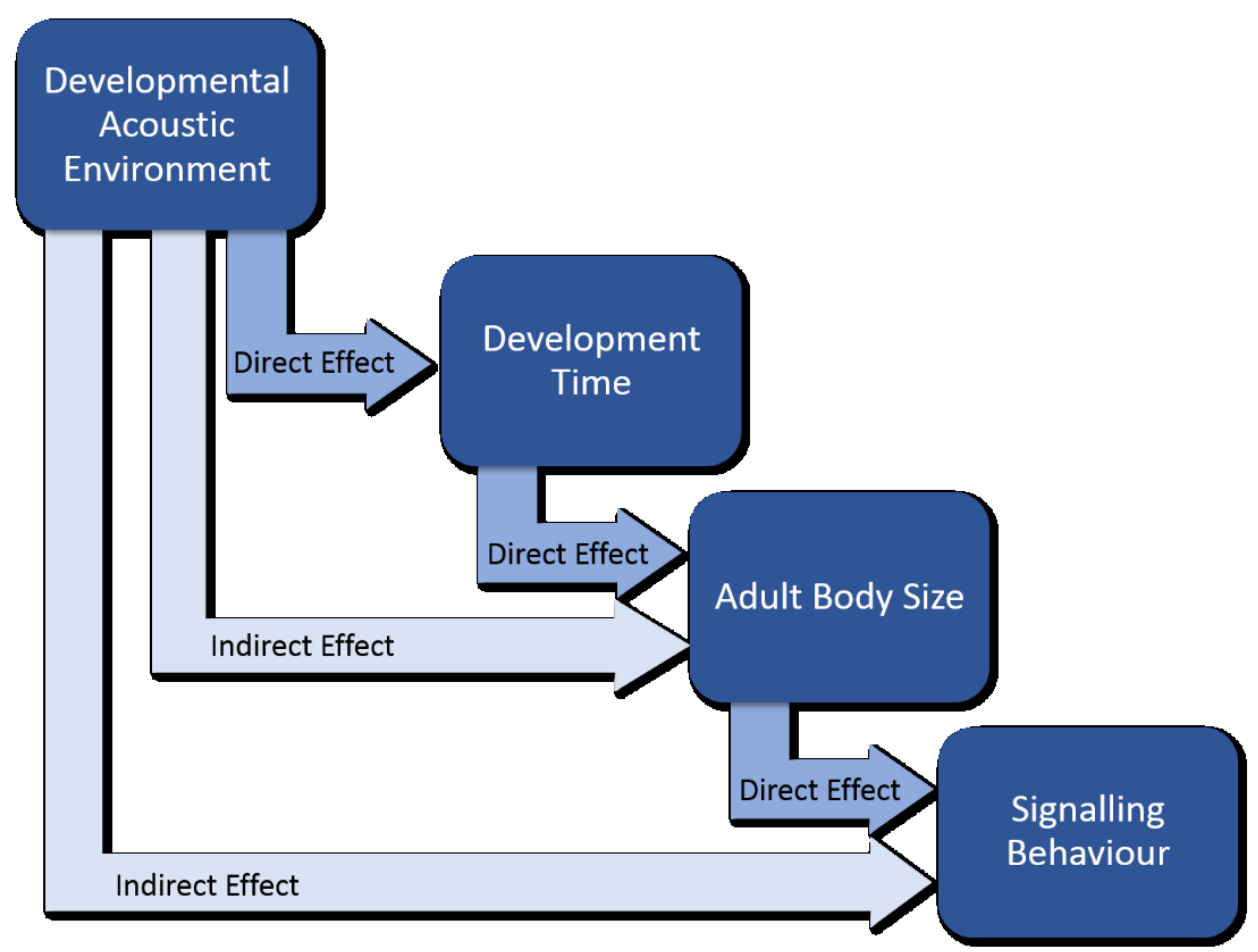

Figure 5.5. Schematic highlighting the indirect effects that rearing acoustic environment can have on adult behaviours through steps of smaller, direct effects on different aspects of life history traits. 
I found no direct effects of anthropogenic noise during development on adult male cricket signals. I did, however, see a near significant effect from the interaction of juvenile signalling density and anthropogenic noise. Specifically, males raised in the Low density environment signalled more often and at higher amplitudes when exposed to the anthropogenic playback. My study was among some of the first to investigate how developing crickets and other acoustically signalling orthopteran respond to anthropogenic noise pollution within their habitat. My finding conflicts with the findings of Costello and Symes (2014), who found that male tree crickets (Oecanthus) collected from within $200 \mathrm{~m}$ of a road were less likely to signal when exposed to additional noise, but the characteristics of their signals remained unchanged. To my knowledge, the only other study to date on the impact of anthropogenic noise during development also revealed different results. Male nymph grasshoppers (Chorthippus bigltulus) reared in the presence of road noise produced adult signals with higher frequency components compared to males reared without noise (Lampe et al. 2014). This finding aligned nicely with the behaviour of adult grasshoppers (Chorthippus bigltulus) from roadside environments, which produce courtship signals at higher frequencies than males from quiet locations (Lampe et al. 2012). Given that I did not observe a shift in frequency like Lampe et al (2014) nor a reduction in signalling like Costello and Symes (2014), but instead saw a non-significant trend towards more signalling at higher amplitudes, future research should examine how anthropogenic noise during development influences adult behaviour to see if an overall consensus can be reached about its impact.

To my knowledge, my research provides the first attempt to examine interaction effects of both social density and anthropogenic noise in Orthoptera and provides the first 
attempt to look at how human noise pollution may be influencing life history and adult behaviour in field crickets. The lack of significant results in my study should not deter further work in this area given that my study crickets were all descendants from individuals gathered from areas that regularly experience anthropogenic noise (Chapter 2). It is possible that my field crickets were genetically predisposed to adjustments in a noisy environment. Lampe et al. (2014) found that nymph grasshoppers transferred from a roadside environment produced adult signals with both higher frequencies and syllableto-pause ratios than nymphs reared from a quiet environment, regardless of developmental treatment (see above). To account for this result, Lampe et al. (2014) proposed that the two populations had genetically diverged. As cricket signals can have a strong genetic component (Gray \& Cade 1999; Tinghitella 2008; Webb \& Roff 1992), future studies should examine differences in individuals from dissimilar environments across time to see if they have also genetically diverged.

Another possible explanation for my finding that anthropogenic noise did not confound the ability of juveniles to accurately eavesdrop on the acoustic social environment is that crickets instantaneously adjust their signalling behaviour in the presence of anthropogenic noise. Orci et al. (2016) revealed that male tree crickets (Oecanthus pellucens) adjust their signals by pausing and shortening their chirp durations when exposed to anthropogenic noise, but only over a short time period and only when the anthropogenic noise reached a threshold decibel level. The researchers hypothesised that pausing and shortening chirp durations were modifications mainly used to conserve energy during signalling at noisy intervals - instead of making costly adjustments to signals such as increasing amplitude or signalling more. As males in my current study 
were necessarily removed from their acoustic environments during signal recording, I was unable to quantify whether males exposed to anthropogenic noise instantaneously adjusted their mate attraction signals in any way. Future work should therefore attempt to quantify whether field crickets adjust their signals at the time of noise playback, and whether this differs when juveniles are exposed to noise pollution during development or reared in silence.

Animal communication exists amidst fluctuations in both ecological and social environments. My study emphasizes how complex these interactions are. My finding that the effects of acoustic social environment during development on male adult signalling were more indirect than direct highlights how whole life experience should be taken into account when studying animal behaviours (French \& Cade 1989; Sih et al. 2004). Furthermore, the interactive effects of anthropogenic noise on adult male signalling underscores the need to consider an individual's entire environmental input, especially in animals whose habitat is increasingly being urbanized. Future work should focus on separating the effects of juvenile versus adult environment to fully understand how developmental constraints control male signal production (which I begin to address in Chapter 7). Additionally, male signalling should be tested under different timing circumstances, to determine if crickets respond differently to instantaneous versus sustained noise pollution. 


\section{Chapter 6: Female field crickets that experience acoustic social signals during development expect more from a mate}

\subsection{Abstract}

In species where females use male sexual advertisements to help make mate choice decisions, males that display energetically costly, high quality signals are often preferred. However, as mate searching and reproduction have their own associated costs, females must balance these costs against the benefits of mating with specific males, and the costs and benefits can drive changes in individual mate preference. When search costs are low, for example when population densities are high and males are plentiful, females can afford to be choosy and less responsive, driving selection towards higher quality males. Conversely, when search costs are high, for example when population densities are low and males are rare, females should be more responsive and less discriminating in their choice, resulting in relaxed selection. Experience plays an important role in helping individual females determine these costs and benefits. This can include experience gained during development through eavesdropping on adult acoustic signals. Anthropogenic noise may interfere with signal transmission and reception by females that rely on acoustic male signals. In this chapter I studied how adult female mating preferences changed in response to adult mate attraction signalling (the acoustic social environment) and noise pollution experienced during development. I raised female fall field crickets, Gryllus pennsylvanicus, while exposing them to one of six different acoustic social environments that differed in the density of signalling adult males (High, Low, and None) and anthropogenic noise (Present or Absent). I then quantified female preference functions and responsiveness using playback experiments with artificial male signals that 
varied in quality of a specific trait (chirp rate). Females from all three juvenile acoustic social environments preferred higher quality male signals (faster chirp rates), however females reared in acoustic social isolation were the most responsive. Additionally, larger females were more responsive than smaller females, with smaller females preferring male signals closer to the population mean. Anthropogenic noise reduced the amount of variation in preference function shape between groups, and females reared with noise pollution tended to have lowered responsiveness to extreme changes in chirp rate compared to females reared without anthropogenic noise. This finding suggests that anthropogenic noise may be interfering with juvenile females' ability to accurately assess adult male signals. Together, these findings support theoretical predictions that juvenile experience is an important factor shaping female mate preference and mate choice and may have implications for the strength and direction of sexual selection on male acoustic mate attraction signals.

\subsection{Introduction}

Female reproductive fitness is dependent on the number of viable offspring they produce. Because reproductive effort is costly, requiring a large allocation of resources, selection should favour traits and behaviours that increase female fecundity and offspring quality while simultaneously reducing the female's costs (Wagner et al. 2001a). Female choice should be one of these behaviours. Females can discriminate between desirable and undesirable mates (Janetos 1980; Darwin 1871). When exposed to one or more males, females must decide which individual(s) to mate with and mate choice decisions can vary between individuals, driving variation in male traits (Jennions \& Petrie 1997). 
A female's assessment of a possible mate may be based on his physical characteristics, the territory he holds, and/or his behaviour. Because these characteristics are often correlated with the male's genetic quality, the benefits received from mating with a high quality male may be direct (e.g. improved territory or parental care) or indirect (genetic benefit and high quality offspring) (Gavrilets et al. 2001; Darwin 1871; Fisher 1930; Zahavi 1975). To gain these benefits, females ultimately have to make a mating decision that is simultaneously weighed against the costs and benefits of continued male sampling. Having ready access to multiple males should lead to choosier females with strong preferences for mating only with males that have high quality attractive traits, as the costs of searching for alternative mates (e.g., energy and time constraints) are lowered (Kokko et al. 2003). If, however, male population densities are lowered, female access to multiple potential mates will also be reduced. Under this scenario, the benefits of exhibiting strong preferences for mating only with males that have attractive high quality traits may not outweigh the costs associated with finding alternative mates - and females can instead increase their fitness by being less choosy (Candolin 2003; Jennions \& Petrie 1997).

There are a plethora of examples that show that female preference (the suite of traits that females find attractive) is directly linked to her environment (e.g. her experience, mate availability, costs of mate searching, predation risks), suggesting that variation in the environment can direct mate choice decisions (Rosenthal 2015). For example, female guppies (Poecilia reticulata) from high predation populations reduce their preference for attractive males in the presence of predators, while females from low predation populations do not (Godin \& Briggs 1996). Female three-spined sticklebacks 
(Gasterosteus aculeatus) use both visual and olfactory cues in mate choice decisions, but the overall influence of these cues is dependent on the condition of the water. In clear water, female sticklebacks rely more on visual cues to choose mates, while in turbid water they rely on olfaction cues, and since these decisions do not always match, differences in the environment can shift mate choice (Heuschele et al. 2009). Females can also alter mate choices depending on the availability of resources. In female black field crickets (Teleogryllus commodus), juvenile diet influences both sexual responsiveness and preference functions. Females raised on high protein diets are more responsive and express stronger preference functions for male signalling traits than females raised on low protein diets, suggesting that female condition and resource acquisition contribute importantly to mate choice decisions (Hunt et al. 2005).

Female mate choice can also be affected by changes to composition of the adult population, either through varying operational sex ratios or overall population densities, both of which may alter female adult social experience (Kokko \& Rankin 2006). For example, preference for a familiar partner in female prairie voles (Microrus ochrogasfer) increases with both mating and cohabitation (Williams et al. 1992). In guppies (Poecilia reticulata), changes in the operational sex ratio influenced female preference for orange colouration, which increased with increasing male-biased operational sex ratio (Jirotkul 1999a). Female guppies are also more likely to mate with a male bearing novel colour patterns than with a male who has familiar colouration (Hughes et al. 1999). In field crickets (Teleogryllus oceanicus) females with prior experience to attractive male signals show reduced preference for a reference signal and are less responsive overall, whereas preference for the reference signal increases in females who experience less attractive 
male signals before their phototaxis trials (Bailey \& Zuk 2009). In another field cricket, (Gryllus pennsylvanicus), inexperienced females (held in isolation) are less choosy than experienced females (held in communal groups) (Judge 2010). Plasticity in female preference functions is thus an important factor allowing females to maintain fitness benefits or to mitigate costs through adaptively altering their behaviours in varying social environments (Rosenthal 2015)

Female mate choice can also be influenced by juvenile social experience. For example, female subadult wolf spiders (Schizocosa uetzi) exposed to adult males with differing phenotypes preferred as adults to mate with males displaying familiar phenotypes, and were more likely to cannibalize males with unfamiliar phenotypes, whereas unexposed females did not discriminate between phenotypes (Hebets 2003). Adult male field crickets use acoustic signalling to advertise their quality to females, and females hearing these signals can discriminate between potential mates or learn about their social demographics (Bigelow 1960; Alexander 1968; Mitra 2011; Judge 2010). Long-distance acoustic mate attraction signals play an important role in female mate choice. Females are able to distinguish between a number of song characteristics such as chirp rate, chirp duration, and signal amplitude, which, as covered in Chapter 5, convey information about male quality (Wagner and Reiser 2000). Kasumovic et al. (2012a) raised juvenile black field crickets, (T. commodus), in different acoustic social environments (levels of male acoustic mate attraction signals) and found that the social environment affected female response speed but not preference. Specifically, females that were exposed to acoustic signalling during development were faster to select the playback with a higher-call rate. Conversely, in another field cricket (T. oceanicus), when 
juvenile females were exposed to male acoustic signals during rearing they were less responsive to male signal during playback tests and they exercised greater discrimination (Bailey \& Zuk 2008). Early experience can therefore affect how females assess the costs and benefits associated with mate choice as adults, ultimately affecting her fitness.

Here I determine how the juvenile acoustic social environment of female Gryllus pennsylvanicus affects their mate preference behaviour in adulthood. Females should be able to use the acoustic environment they are exposed to as juveniles to judge their future population demographics and hence the density of potential mates, impacting their mate preferences in adulthood. I predict that females who are exposed to male acoustic mate attraction signalling during development will be more selective in their mate choice upon maturation. Alternatively, I predict that adult females who have had little or no exposure to acoustic signalling during development will likely respond more quickly to male signals and be less discriminating because they may perceive that their environment lacks potential mates and they may not want to risk losing a mating opportunity.

Male advertisement signals have the potential to be affected by anthropogenic noise pollution. Urban noise pollution has been shown to affect acoustic signalling in copious species, including red-winged blackbirds (Agelaius phoeniceus, Hanna et al. 2011), European robins (Erithacus rubecula, Montague, Danek-Gontard, and Kunc 2012), European tree frogs (Hyla arborea, Lengagne 2008), tree crickets (Oecanthus pellucens, Orci, Petr, and Barta 2016), and grasshoppers (Chorthippus biguttulus, Lampe et al. 2012). A growing number of studies have also found that anthropogenic noise can impact female phonotaxis and thus response to male acoustic signals (Gurule-Small \& Tinghitella 2018; Schmidt et al. 2014; Costello \& Symes 2014; Bee \& Swanson 2007). 
Therefore, in addition to juvenile acoustic social environment, here I establish how the presence of anthropogenic noise, specifically urban noise pollution, interferes with the female's ability to properly assess the expected cohort of potential mates, resulting in different mate-choice behaviour upon maturity. I predict that anthropogenic noise pollution will mask the acoustic signals, making it difficult for the juvenile females to accurately predict the density and quality of future mates. This may result in females expecting fewer mating opportunities at adulthood resulting in adults that are more responsive to male signal playback.

\subsection{Methods}

\subsubsection{Study Species and Cricket Rearing}

I used laboratory-reared North American Fall Field crickets, G. pennsylvanicus, in my study. To test how developing in different acoustic social environments influences female mate choice in adulthood, I individually housed one-week old juveniles and then randomly assigned them to one of 6 acoustic environment treatments: 3 with different densities of male acoustic mate attraction signals (High, Low, None), and 3 with these acoustic signals overlaid with anthropogenic noise (Table 2.1; Figure 2.3). Crickets in each treatment were housed together in a larger communal treatment bin lined with acoustic foam. Each treatment bin contained 4 speakers for playback of the treatment's acoustic environment soundtracks. Thrice weekly, I checked each juvenile cricket for wing pad eclosion or adult eclosion.

Once female crickets underwent their imaginal adult moult, I gave them an experimental ID, weighed them (Pioneer Balance model PA214, OHAUS Corporation, 
USA), placed them each in a new individual container ( $\mathrm{L} \times \mathrm{W} \times \mathrm{H}=10 \times 7 \times 7 \mathrm{~cm})$, and then returned them to the same communal treatment bin they experienced during development. Female crickets do not create acoustic signals, so adult females could remain in the juvenile communal treatment bins without causing potential acoustic interference, unlike adult males (detailed in Chapters 4 and 5). Adult females remained in these communal treatment bins until they were $14 \pm 2$ days post imaginal moult. I then quantified each female's mating preference function for chirp rate by placing them on a spherical treadmill and quantifying their movement towards artificial calls broadcast from surrounding speakers (phonotaxis). Below I describe how I created the artificial signals, quantified female phonotaxis, and quantified female preference functions.

Chapter 2 provides a detailed overview of how I produced my six different acoustic treatments, how I housed my individual crickets during rearing in their different acoustic treatments, how I quantified body size, and the other general protocols that I used for all data chapters.

\subsubsection{Artificial Signal Production}

To test the effect of juvenile rearing environment on adult female preference I ran phonotaxis trials using artificial male long-distance acoustic mate attraction signals that varied only in chirp rate. Chirp rate was selected as the parameter to alter because natural changes in the number of chirps per minute varies with male condition and thus can influence female choice (Whattam \& Bertram 2011; Wagner \& Hoback 1999). Faster chirp rates require more energy to produce and females tend to prefer males that signal at faster chirp rates (Wagner 1996; Wagner \& Reiser 2000; Walikonis et al. 1991). While 
some evidence indicates that chirp rate may be manipulated in dishonest signalling, where smaller males produce signals with a faster chirp rate than larger males (Harrison et al. 2013), female G. pennsylvanicus still show a directional preference for higherenergy signals (Judge 2008).

I chose to use artificial signals over natural signals to ensure that all signal parameters other than chirp rate were identical and represented an average male cricket. To create the artificial signals, I recorded a sample population of 36 lab-reared, 7-10 day old virgin male G. pennsylvanicus using the Electronic Acoustic Recording System (EARS II, Cambridge Electronic Design, Cambridge, U.K.; see Chapter 5 for more details on this system). Male signals were recorded continuously for 4 days at $25 \pm 2^{\circ} \mathrm{C}$. I then calculated sample population mean values using Spike2 v 6.09 (Cambridge Electronic Design, Cambridge, U.K) for several components of the male signal (components described in Figure 2.2), including: pulse duration (ms), inter-pulse duration (ms), chirp duration (ms), inter-chirp duration (ms), number of pulses per chirp, carrier frequency $(\mathrm{Hz})$, amplitude $(\mathrm{dB})$, pulse rate (\#pulses/sec), and chirp rate (\#chirps/min) (Table 6.1). 
Table 6.1. Mean and standard deviation values for long-distance mate attraction signal parameters of the sample population used to create the artificial signals.

\begin{tabular}{lrr}
\hline Signalling Component & Mean & SD \\
\hline Pulse Duration (ms) & 15.53 & 2.64 \\
Inter-Pulse Duration (ms) & 36.42 & 2.40 \\
Pulses Per Chirp & 3.25 & 0.51 \\
Chirp Duration (ms) & 97.33 & 16.66 \\
Inter Chirp Duration (ms) & 836.81 & 302.75 \\
Amplitude (db) & 56.24 & 8.09 \\
Carrier Frequency (Hz) & 4954.34 & 244.27 \\
Pulse Rate (\#Pulses/sec) & 19.32 & 1.18 \\
Chirp Rate (\#Chirps/min) & 69.84 & 19.00 \\
\hline
\end{tabular}


To produce the artificial signals, I first examined a subset of the recorded sample signals to quantify within-chirp variation using Adobe Audition CS6 (version 5.0). This allowed me to mimic natural variation in parameters such as pulse duration, inter-pulse duration, and pulse amplitude. I downloaded sound pulses that represented the population's average carrier frequency and pulse duration from an online tone generator, Wavtones (www.wavtones.com). Using Adobe Audition, I then manipulated the amplitude of each pulse to match the amplitude of each pulse in an average chirp. I then created a single chirp by stringing these ordered pulses together using the appropriate inter-pulse durations to ensure that the signalling parameters in the chirp I created matched the population averages. I then produced each specific chirp rate's acoustic mate attraction signals by concatenating chirps together using specific inter-chirp durations. I used this approach 15 times, to create a total of 15 different artificial signals that varied only in their chirp rates. These final 15 artificial signals were therefore identical in all parameters, with each of these parameters matching the sample population's means (Table 6.1), with the exception of chirp rate. One of the signals had a chirp rate representative of the population mean. The other 14 differed from the mean population chirp rate by $-3.5,-3,-2.5,-2,-1.5,-1,-0.5,+0.5,+1,+1.5,+2,+2.5,+3$, or +3.5 standard deviations (SD) away from the mean. Thus, the chirp rate represented by $-3.5 \mathrm{SD}$ was an exceptionally slow chirp rate, while the chirp rate represented by the $3.5 \mathrm{SD}$ was an exceptionally fast chirp rate. Each of these artificial signals was 1 minute in duration. Finally, I also created a one-minute long pure tone signal as a control. Each female was thus tested on 16 different signals, a standard mean signal, 14 signals with chirp rates above and below the population mean, and a control signal that was a pure tone played at 
the same amplitude and carrier frequency as the other 15 signals.

\subsubsection{Phonotaxis Trials}

As female crickets are sexually mature and exhibit the highest phonotaxis to acoustic mate attraction signals when they are between 10 and 16 days of adulthood (Pacheco et al. 2013), I quantified adult female mate preference behaviour by measuring their phonotaxis to these 16 different artificial signals when the females were $14 \pm 2$ days post imaginal moult. At 12 days post imaginal moult, I weighed each female and then attached a dental elastic (3.2mm diameter, Henry Schein, Inc) to their pronotum using standard fast-cooling wax (Strip-free Hot Wax, Parissa Laboratories Inc.). I then returned the female to their individual container located in their communal acoustic social environment treatment until their phonotaxis trial two days later.

I ran all phonotaxis trials using a spherical treadmill apparatus placed in an acoustically isolating chamber $(142 \times 79 \times 96.5 \mathrm{~cm}, \mathrm{~L} \times \mathrm{W} \times \mathrm{H})$ to limit observer disturbance. The treadmill consisted of 4 main components (Figure 6.1): (1) a lightweight polystyrene ball $(12.7 \mathrm{~cm}$ in diameter) floating on cushion of air and contained within (2) a cowling designed to house the ball and maintain constant airflow, (3) an electronic sensor to detect rotation of the ball, and (4) a software program to record sensor readings and calculate precise movements of the ball (sensu Pacheco et al. 2013). An external air compressor (King Industrial - Ultra-Quiet Oil-Free Air Compressor - KC4620A) provided controlled air flow through tubing attached to the bottom of the cowling that raised the polystyrene ball on a cushion of air (0.5 - 2 PSI). I housed the air compressor in a separate room to reduce noise interference. I illuminated the chamber using low intensity LED light sources $(3.6 \mathrm{~W}, \mathrm{AC} 120 \mathrm{~V})$ with a red light filter (Roscolux 
R27 - medium red; Rosco, Sydenham, London, UK) which filtered out most light wavelengths that Orthopteran visual receptor cells are sensitive to $(<600 \mathrm{~nm}$; Briscoe and Chittka 2001), thereby simulating dim lighting. Speakers (Insignia NS-PCS40; Richfield, MN, USA) were mounted on either side of the chamber $54 \mathrm{~cm}$ from the center of the treadmill where the female was mounted. Each signal-speaker combination was calibrated to play at $56 \mathrm{~dB}$ (mimicking the sample population mean amplitude, Table 6.1). Amplitude calibration measurements were taken at the top of the polystyrene ball using a sound pressure level meter (Extech Instruments Model 407750; Nashua, NH, USA) with the microphone pointed directly at the speaker. Trials were monitored using a GoPro HERO4 Silver camera (GoPro Inc. USA) mounted $22 \mathrm{~cm}$ away from the cricket, which could be remotely controlled with a tablet GoPro app on a tablet or smart phone (Figure 6.1). 


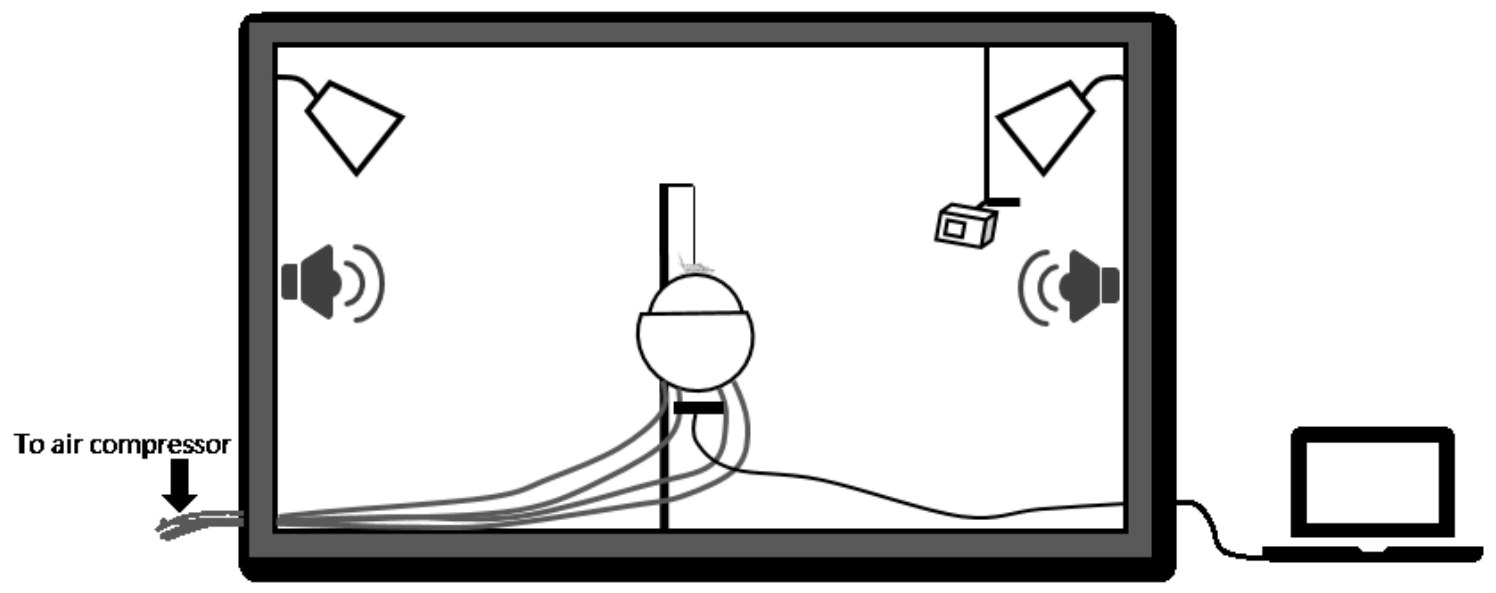

Figure 6.1. Phonotaxis trial apparatus set-up detailing placement of speakers, lights, GoPro, and spherical treadmill within the sound attenuating container. Air tubes are observed exiting the container to an external air-compressor. 
On the day of their phonotaxis trials, females were centered on the top of the polystyrene ball and held in place by attaching the elastic on their pronotum to a fish hook attached to a telescoping arm centered above the treadmill (Figure 6.2). The height of the telescoping arm was carefully adjusted to ensure that the female was standing in a natural position on the treadmill. Air pressure was then adjusted so that the polystyrene ball was able to rotate freely, such that the cricket could rotate the ball without restrictions as she walked or ran. Once the female was properly positioned on the polystyrene ball and able to move freely, the door to the sound attenuating chamber was closed and the female was given a 2 minutes silent acclimatization period. During this time, she was observed via the GoPro to ensure she remained able to freely move without agitation. After the silent acclimatization period, females were presented with a series of 17 phonotaxis signals, each lasting one minute. The first trial consisted of the standard mean signal, presented through a single speaker. The side of the initial speaker was selected at random and then alternated for the remaining 16 signals. The second signal provided to the female was the pure tone, followed by a randomized presentation of the 14 phonotaxis signals of differing chirp rates, and ending with the standard mean signal. Each female's locomotor behaviour on the treadmill was quantified at a sample rate of 20 samples per second, resulting in a total of $1200 \mathrm{X}$, Y position samples for each artificial signal. Females were given 30 seconds of silence between consecutive signals, resulting in phonotaxis trials that lasted approximately 25 minutes in total. All trials took place between 08:00 and 17:00 $\mathrm{H}$, at $21-27^{\circ} \mathrm{C}\left(\mathrm{SE}=0.10^{\circ} \mathrm{C}\right)$. After their phonotaxis trials, females had the elastic removed from their pronotum and were euthanatized via freezing. Please see Chapter 2 for details on photography and body size measurements. 


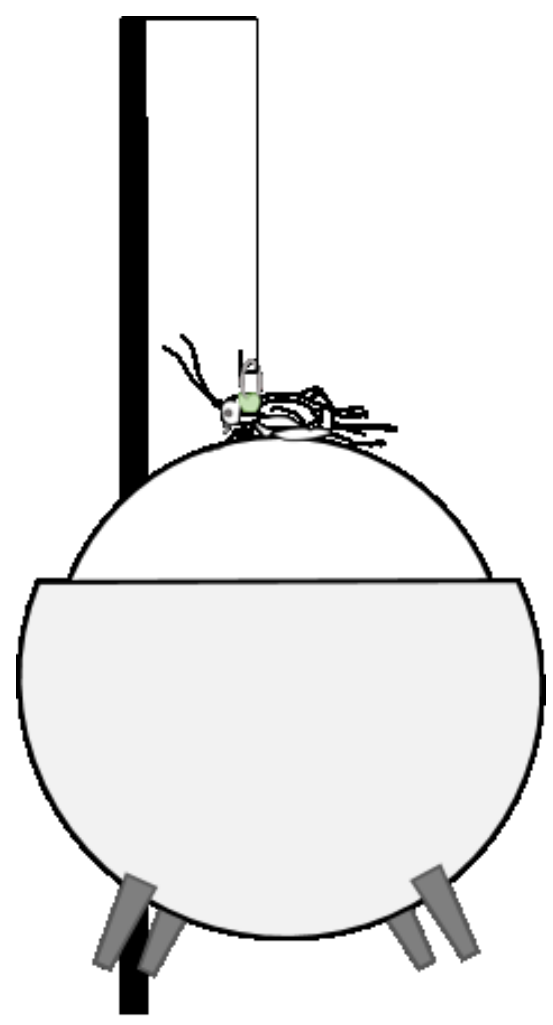

Figure 6.2. Detail of spherical treadmill within cowling with mounted female cricket. The design of the elastic hook apparatus on the female's pronotum allowed her to freely rotate and run on the treadmill with little to no resistance during phonotaxis trails. 
Custom software created in MATLAB (ver 9.1; MathWorks, Natick, MA, USA) detected movement of the treadmill and converted it to displacement of the female in $\mathrm{cm}$. From this, the software calculated instantaneous displacement $(\mathrm{cm})$ and velocity $(\mathrm{cm} / \mathrm{s})$ vectors from the $\mathrm{X}, \mathrm{Y}$ coordinates. To determine female preference functions, I calculated Net Vector scores for each 1-minute trial of the female's net movement towards or away from the active speaker using the following equation (Pacheco et al. 2013):

$$
\text { Net Vector Score }=\sum_{t=1}^{1200}[\cos (\text { vector angle }(t)) \mathrm{x} \text { vector length }(t)]
$$

Net vector score thus considers the females direction (vector angle) and vector length for every recorded movement. As the angle of the active speaker is $0^{\circ}$, females moving towards the speaker had a positive net vector score and females moving away from the speaker had negative scores. The size of the score reflects how quickly each female was moving. A large positive score indicates a female moved quickly towards the active speaker while a large negative score indicates a female moved quickly in the opposite direction away from the speaker (Huber et al. 1984; Pacheco et al. 2013). Thus net vector score can be used as a reliable indicator of female responsiveness as it takes into account her orientation towards the active speaker as well as her motivation (speed) to move towards (or away from) the presented signal (Pacheco et al. 2013).

To understand how the acoustic social environment treatment influenced female preference functions, I had to first visualize the shape of the preference function for each female. To do this I used the software program Pfunc to plot each female's net vector score for the different chirp rates (Kilmer et al. 2017). Pfunc fits a non-parametric curve 
to the female's data using generalized additive models, and then extracts five traits from each female's preference function. These Pfunc traits describe the height and shape of the curves: 1) peak height - the maximum preference response at the preferred stimulus; 2) tolerance - the range of artificial signals to which females still exhibit a strong response within a preference curve (about 1/3 of peak height); 3 ) strength - the degree to which preference drops away from peak preference (the most preferred chirp rate); 4) responsiveness - the average preference score across all artificial signals; and 5) peak stimulus - the artificial signal with the most preferred chirp rate (Kilmer et al. 2017). Thus, because Pfunc produced key traits that describe each female's preference function, I could then determine whether these preference function traits differed depending on the acoustic social environment treatment that the female developed in.

\subsubsection{Statistical Analysis}

All statistical analyses were performed using R (3.4.3 Kite-Eating Tree). Because four of the traits extracted by Pfunc were correlated (peak height, tolerance, strength, and responsiveness), I performed a Principle Component Analysis (PCA) to extract orthogonal vectors and then performed varimax rotation on the first 2 principle components (Table 6.2). The first two rotated principle components were selected (preference factor 1 and 2) as they had eigen values above 1 (Kaiser 1964), satisfied the scree test for factor rotation conditions (Cattell 1966), and together explained $91 \%$ of the variation in female preference traits extracted by PFunc. Preference factor 1 (Eigenvalue $=2.20$ ) explained $55 \%$ of the preference variation and was indicative of how responsive a female was: as high scoring females had higher net vector scores, travelled faster and farther towards a signal (greater peak height and responsiveness). Preference factor 2 
(Eigenvalue $=1.43)$ explained $36 \%$ of the preference variation and was indicative of how choosy females were: females with a high score were less choosy (lower strength) and had greater tolerance across the range of artificial signals (Table 6.2).

I used generalized linear models (GLM's) to examine the effects of the acoustic environment during development on adult female's preference factor 1 (family = gamma, link $=$ inverse) and factor 2 (family $=$ gaussian, link $=$ identity). Both models included the acoustic social environment treatments associated with signalling density (None, Low, High), anthropogenic noise (No, Yes), and their interaction as independent variables. I also included residual mass at adulthood, body size (PC1 size), and season (Spring, Summer, Fall, Winter) as covariates in the model. 
Table 6.2: Rotated PCA factor loading values for preference factor 1 and 2 of four preference traits after varimax roation. Signs $(+/-)$ on loadings indicate that higher levels of a factor are positively or negatively associated with that parameter. Loadings above 0.600 are shown in bold.

\begin{tabular}{lrr}
\hline Preference Traits & Preference factor 1 & Preference factor 2 \\
\hline Eigenvalue & 2.20 & 1.43 \\
Percent & 55.03 & 35.81 \\
Cumulative Percent & 55.03 & 90.83 \\
Peak Height & $\mathbf{0 . 9 9 6}$ & 0.034 \\
Tolerance & 0.033 & $\mathbf{0 . 9 6 3}$ \\
Strength & -0.131 & $\mathbf{- 0 . 6 6 0}$ \\
Responsiveness & $\mathbf{0 . 9 7 8}$ & 0.195 \\
\hline
\end{tabular}


While Pfunc allows me to extract traits that nicely describe the female preference function, one of the drawbacks to PFunc is that I cannot test for whether the order the chirp rate was played influenced female phonotaxis. Therefore, to ensure the effect of trial order was accounted for I also ran a generalized linear mixed model (family = Guassian, link = Identity) with individual as a random effect. There are pros and cons to this approach. While the GLMM allows me to test for order effects, it is more limited than the Pfunc approach because it mandates that the female's NVS response to different chirp rates must be either linear or quadratic. I therefore quantified female preference functions using both approaches, as strongly encouraged by Kilmer et al. (2017). For my GLMM I used net vector scores as the dependent variable. The independent variables were the acoustic social environments associated with signalling density (None, Low, High) and anthropogenic noise (Yes, No), trial order (linear and nonlinear terms), adult body size (PC1 size), residual mass at adulthood, season (Spring, Summer, Fall, Winter), chirp rate standard deviation (linear and nonlinear terms) and the interactions between chip rate and signalling density, chirp rate and body size, chirp rate and anthropogenic noise, and chirp rate and season. Season of hatching was included in the models as a block effect to control for possible environmental changes throughout the year.

I selected error families and link functions for all statistical models that minimized residual deviance, I also visually examined the quantile-quantile and residual plots using R's “DHARMa” package (Hartig 2017). For all models, I checked for multicollinearity between main effects using the "usdm" package (Naimi et al. 2014). I found minimal multicollinearity in all models, as all variance inflation factors were $<1.5$ (values > 10 indicate multicollinearity; (Naimi et al. 2014). For all models, I performed 
model simplification by removing the non-significant interaction terms beginning with the highest-order interactions. I used deletion tests with the anova function in $\mathrm{R}$ to determine if the removal of the term significantly improved the model fit. Based on the number of overall effects in the saturated models, I corrected for multiple hypotheses

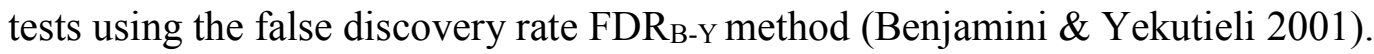

\subsection{Results}

I analyzed preference functions of 187 females using their phonotaxis behaviour towards 15 different signals that varied in chirp rate while holding all other mate attraction signalling parameters constant. The 187 adult females were reared in the following acoustic social environment treatments: High density: Anthropogenic noise $=$ 30, No Anthropogenic noise $=31$; Low density: Anthropogenic noise $=33$, No Anthropogenic noise $=32$; Isolation: Anthropogenic Noise $=29$, No Anthropogenic noise $=32)$.

\subsubsection{Preference Function Shape}

Overall, female preference functions increased linearly with increasing chirp rate values (Figure 6.3). However, visual examination suggests that the acoustic social environment treatment the females developed in tended to influence female preference functions. Females reared in the silent treatment (None), with no signalling input, were the most responsive (had the highest net vector score responsiveness), followed by females reared in the Low and then High signalling density treatments (Figure 6.3 A). When male signalling densities were combined with anthropogenic noise, there was more overlap between all three density groups (Figure 6.3, B). Below I explore the statistical 
significance of these visual observations.

\subsubsection{Preference Function Traits}

Exposure during development to different acoustic social environment treatments (signalling densities and/or anthropogenic noise) did not significantly affect adult female preference Factor's 1 or 2 (Table 6.3). However, there was a near significant effect $(P=$ 0.039 ) of signalling density where females reared in the None treatment tended to have higher preference Factor 1 scores than females reared in either High or Low treatments, and were thus generally more responsive (Figure 6.4, B). There was also a nonsignificant trend for preference Factor 2, where females reared in the None treatment tended to have higher scores than females reared in High or Low treatments, meaning they tended to be more tolerant across the range of artificial chirp rates and less choosy (Figure 6.5; Table 6.3). Females that were larger at adult eclosion also had significantly higher preference Factor 1 scores than smaller females, with larger females being more responsive than smaller females $(P=<0.0001)$ (Figure 6.4 A; Table 6.3). 


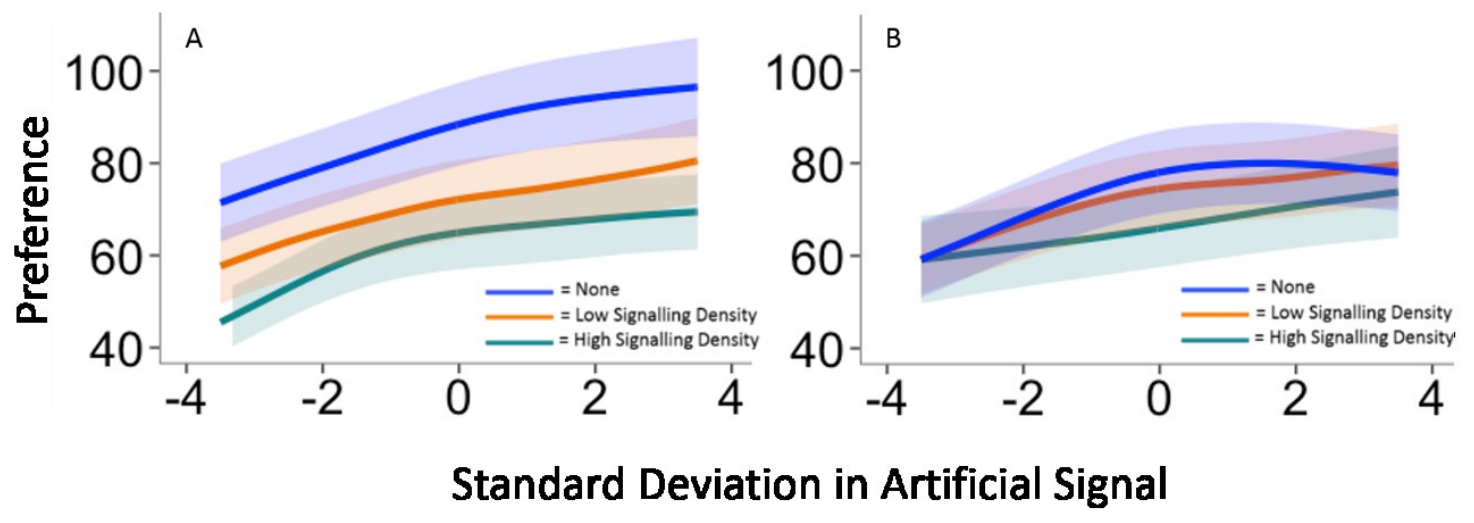

Figure 6.3. Average preference functions (solid lines) with standard error bars for females who developed in each acoustic social environmental treatment, for A) different signalling densities without anthropogenic noise), and B) different signalling densities with anthropogenic noise, fitted with Pfunc. 
Table 6.3. Model effects of the generalized linear model examining effects of acoustic treatment, residual mass, adult body size (PC1 Size), and season on female preference Factor 1 and 2. Significant effects are bolded. FDR $\mathrm{BY}_{\mathrm{B}}$ corrected level of significance is $P$ $=0.022$. Parameter estimates are included in Appendix 5, Table A5.1

\begin{tabular}{llrrr}
\hline Model & Model Effect & $\begin{array}{r}\text { LR } \\
\text { Chisq }\end{array}$ & Df & Pr(>Chisq) \\
\hline Preference Factor 1 & Signalling Density & 6.49 & 2 & 0.039 \\
& Anthropogenic Noise & 0.02 & 1 & 0.89 \\
& Residual Mass & 1.68 & 1 & 0.19 \\
& PC1 Size & $\mathbf{2 0 . 3 9}$ & $\mathbf{1}$ & $<\mathbf{0 . 0 0 0 1}$ \\
& Season & $\mathbf{1 5 0 . 5 1}$ & $\mathbf{3}$ & $<\mathbf{0 . 0 0 0 1}$ \\
Preference Factor 2 & Signalling Density & 5.06 & 2 & 0.08 \\
& Anthropogenic Noise & 0.60 & 1 & 0.44 \\
& Residual Mass & 1.05 & 1 & 0.31 \\
& PC1 Size & 0.63 & 1 & 0.43 \\
& Season & 1.93 & 3 & 0.59 \\
\hline
\end{tabular}



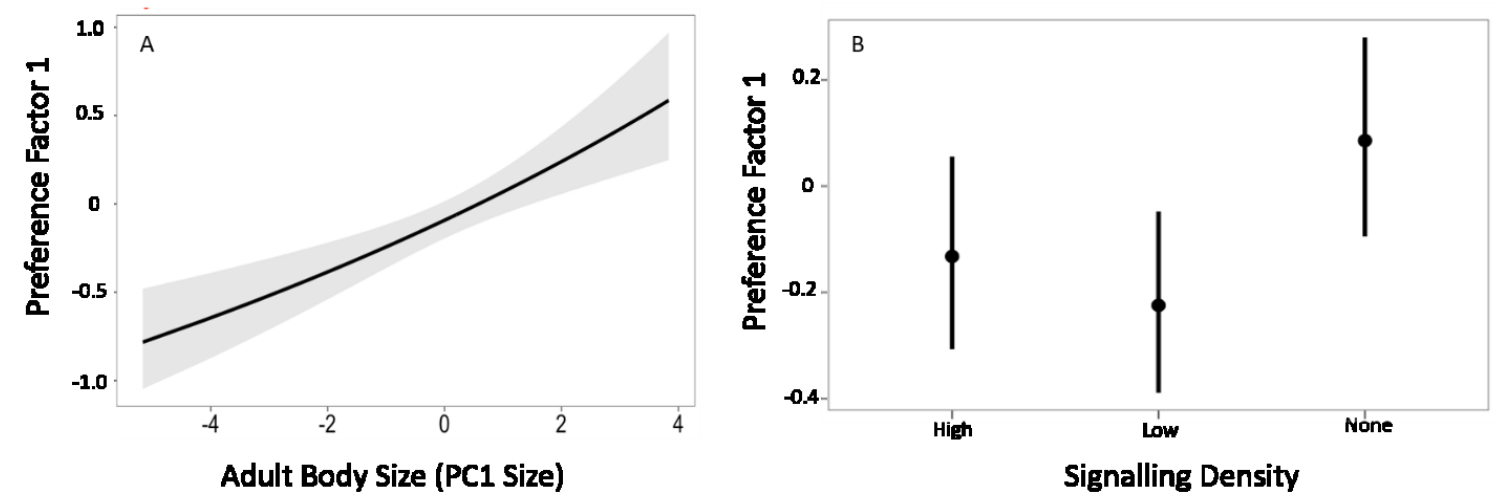

Figure 6.4. Marginal effect plots with 95\% confidence intervals showing how A) adult body size and B) signalling density (near-significant) during development effect responsiveness (Preference Factor 1) to the artificial signals. 


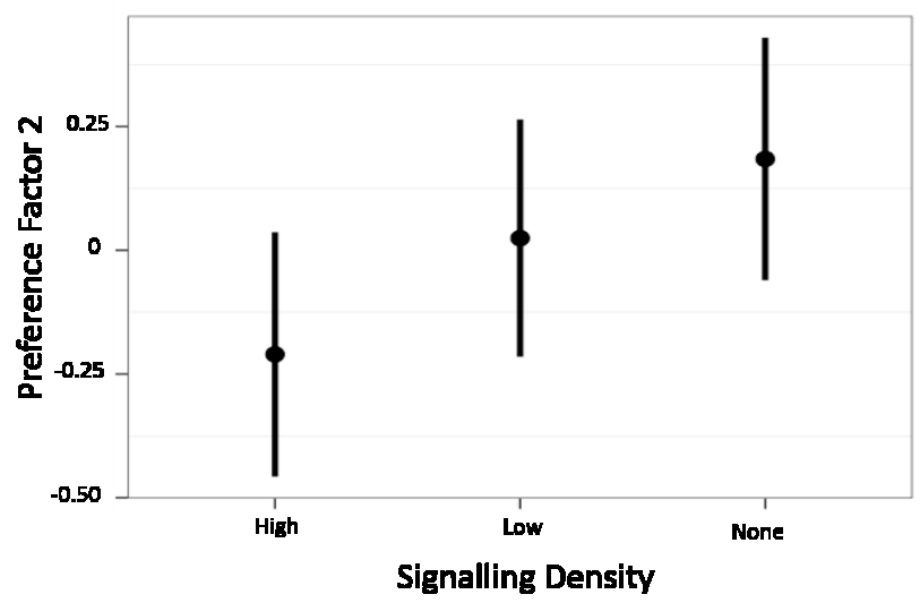

Figure 6.5. Marginal effect plots with $95 \%$ confidence intervals showing how signalling density during development effect choosiness (Preference Factor 2) to the artificial signals (non-significant result). 


\subsubsection{Net Vector Scores}

Quantifying the factors that influenced female net vector scores (Table 6.4) revealed similar results to the Pfunc preference trait analyses, except that the non-significant trends from the Pfunc analyses were now significant in the GLMM after accounting for variation due to the timing of when the different chirp rates were played. For example, I found that female net vector scores were influenced by a significant interaction between chirp rate and signalling density. Adult females from all three juvenile acoustic social environments (None, Low, and High) were more responsive to higher chirp rates $(\mathrm{P}=$ 0.0037) (Figure 6.6, A). Similar to the preference function traits described in the previous paragraphs, females reared in the None environment had the highest net vector scores overall and these net vector scores were significantly higher than females that were reared in the High and Low environment (Tukey's test, $P=0.0036$ and $P<0.0001$, respectively). While females reared in the High environment appeared to have intermediate net vector scores instead of lower net vector scores, it is important to note that the net vector scores of females reared in the High environment did not significantly differ from the net vector scores of females reared in the Low environment (Figure 6.6, A; Tukey's test, $\mathrm{P}=0.4258$ ). The differences in responsiveness between treatments also increased with increasing chirp rates (Figure 6.6, A).

Female net vector scores were also influenced by a near significant interaction between chirp rate standard deviation and anthropogenic noise $(P=0.053$; Figure 6.6B). Specifically, females reared in the presence of anthropogenic noise tended to display a more curvilinear response to increasing chirp rate and also tended to have lower net vector scores for more extreme chirp rates (higher standard deviations) when compared 
with females who were reared without exposure to anthropogenic noise (Figure 6.6, B).

I also found that female net vector scores were influenced by a significant interaction between chirp rate and adult body size. Specifically, larger females were more responsive to higher chirp rates than smaller females. Larger female's net vector scores increased linearly with higher chirp rates $(\mathrm{P}=0.0003)$ (Figure 6.6, C). Conversely, smaller female's net vector scores had a more curvilinear response to increasing chirp rate, with the highest response at the mean chirp rate value. Net vector score was not significantly affected by the trial order, as responsiveness did not drop significantly towards the end of the trials. 
Table 6.4. Model effects of the generalized linear model examining the effects of trial order, adult body size (PC1 size), residual mass, season, acoustic treatment, and chirp rate on female net vector scores. Significant effects are bolded. FDR $\mathrm{BY}$ corrected level of significance is $P=0.015$. Parameter estimates are included in Appendix 5, Table A5.2.

\begin{tabular}{lrrr}
\hline Model Effect & Chisq & Df & Pr(>Chisq) \\
\hline Order & 0.88 & 1 & 0.35 \\
Order (nonlinear) & 2.13 & 1 & 0.14 \\
PC1 Size & $\mathbf{1 0 . 9 2}$ & $\mathbf{1}$ & $\mathbf{0 . 0 0 1 0}$ \\
Residual Mass & 0.53 & 1 & 0.47 \\
Season & $\mathbf{5 5 . 6 0}$ & $\mathbf{3}$ & $<\mathbf{0 . 0 0 0 1}$ \\
Signalling Density & $\mathbf{2 1 . 8 7}$ & $\mathbf{2}$ & $<\mathbf{0 . 0 0 0 1}$ \\
Anthropogenic Noise & 0.05 & 1 & 0.83 \\
Chirp Rate (standard deviation) & $\mathbf{2 2 2 . 2 0}$ & $\mathbf{1}$ & $<\mathbf{0 . 0 0 0 1}$ \\
Chirp Rate (standard deviation)(non-linear) & $\mathbf{1 6 . 5 4}$ & $\mathbf{1}$ & $<\mathbf{0 . 0 0 0 1}$ \\
PC1 Size*Chirp Rate (standard deviation) & $\mathbf{1 3 . 2 1}$ & $\mathbf{1}$ & $\mathbf{0 . 0 0 0 3}$ \\
Season*Chirp Rate (standard deviation) & $\mathbf{3 0 . 0 0}$ & $\mathbf{3}$ & $<\mathbf{0 . 0 0 0 1}$ \\
Signalling Density * Chirp Rate (standard deviation) & $\mathbf{1 1 . 2 1}$ & $\mathbf{2}$ & $\mathbf{0 . 0 0 3 7}$ \\
Anthropogenic noise*Chirp Rate (standard deviation)(non-linear) & 3.76 & 1 & 0.053 \\
\hline
\end{tabular}




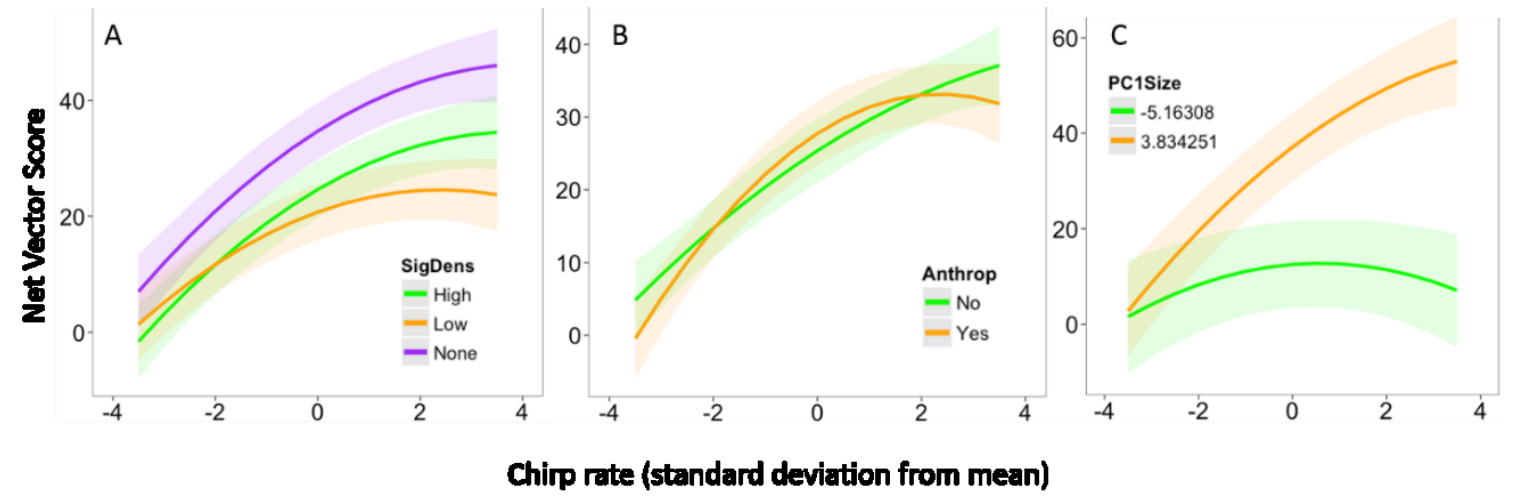

Figure 6.6. Marginal effects plots with $95 \%$ confidence intervals showing how female preference (net vector score) was influenced by interactions between the standard deviation of chirp rate within the artificial signals and A) the signalling density during development, B) the presence of anthropogenic noise (near significant), and C) adult body size at the two extremes. 


\subsection{Discussion}

I tested the hypothesis that while G. pennsylvanicus females should prefer more energetically costly signals (higher chirp rates), female mating preferences should be influenced by the acoustic environment in which they were reared as juveniles. I hypothesized that the acoustic social environment should influence female mating preferences as adults, because the decision to mate or not with a male should be balanced by the constraints associated with lost time/energy from searching for alternative mates. When the density of potential mates is high, females should experience minimal time/energy costs from avoiding less preferred mates and instead search for more preferred mates. Given this, females should be choosier when the density of potential mates is high, and should be less choosy and more responsive when the density of potential mates is low.

To test my hypothesis, I manipulated the density of conspecific acoustic signals females were exposed to during their development and then tested their preference functions when they were 2 weeks post imaginal moult. I found support for most of my predictions, as the signalling density females were reared in either had significant affect or there were non-significant trends. I was also interested in ascertaining whether exposure to anthropogenic noise would obfuscate the female's ability to detect the density of potential mates and respond accordingly. Anthropogenic noise tended to mask the female's ability to detect the signals of conspecific males.

My findings align with other studies that reveal females regularly eavesdrop on signalling males to assess both male quality and availability, and alter their mating preferences accordingly (Sih et al. 2009). For example, female great tits (Parus major), 
eavesdrop on male song interactions to evaluate male quality in adjacent territories and change their mating patterns according to male performance (Otter et al. 1999). Female fighting fish (Betta splendens) watch male aggressive contests and preferentially display towards winners over losers (Doutrelant \& McGregor 2000). Furthermore, juvenile Australian black field cricket (T. commodus) exposed to different population densities during rearing developed different levels of responsiveness (Kasumovic, et al. 2012 a).

\subsubsection{Overall Preference Functions}

Female preference function shapes for chirp rate were generally linear, with preference increasing as chirp rate increased in the artificial signals. These results are consistent with previous findings indicating that female crickets prefer males who signal with higher chirp rates that are energetically costly to produce (Simmons 1988; Wagner \& Reiser 2000; Scheuber et al. 2004; Hirtenlehner \& Römer 2014; Pollack \& Hoy 1981).

\subsubsection{Impact of Acoustic Social Environment on Adult Female Preference}

I found support for my prediction that females raised in social isolation (None environment) should be more phonotactic to acoustic mate attraction signals than females raised in the presence of adult male signalling (Low and High environments). While this finding was only near-significant for the preference function traits (Table 6.3), females in the None environment responded with significantly higher net vector scores across all artificial signals (Table 6.4). There was also a non-significant trend for females in the None environment to be less choosy (preference function 2) suggesting both a greater response and higher tolerance for variation in chirp rate when females have no previous social experience. Research regarding female cricket mate choice in response to juvenile social environment is growing (Kasumovic, et al. 2012 a; Bailey \& Zuk 2008; Bailey \& 
Macleod 2014; Rebar et al. 2016; Swanger \& Zuk 2015) but more focus has been placed on changes within adult social environment. Adult females that are able to gather social information are capable of altering their mating decisions based on experience (Bailey \& Zuk 2009), vary reproductive investment (Conroy \& Roff 2018), and display differing levels of responsiveness (Atwell \& Wagner 2014; Lierheimer \& Tinghitella 2017; Wagner et al. 2001b; Swanger \& Zuk 2015). Across these studies, findings suggest that females exposed to social information, either through mating, population densities, or acoustic experience, are more likely to show stronger preferences for attractive traits and to be less responsive overall. Discriminate mating tactics can be advantageous to females when the cost of making a choice is low. When females perceive multiple mating opportunities, it is in their best interest to adopt mating tactics that allow for increased fitness either through direct benefits (resources, territory, sperm quantity) or indirect benefits (higher-quality offspring, e.g., "sexy sons", Weatherhead and Robertson 1979). Females reared in the Low and High environments were exposed to male signals, and therefore could have perceived high enough availability of males to have multiple mating opportunities at adulthood. These females were thus less responsive. Conversely, females that were reared in social isolation (None environment) were provided with no information concerning the potential quality and availability of future mates. These females could have perceived low availability of potential mates. As a result, they were more responsive adults, running faster towards the speaker broadcasting conspecific male mate attraction signals at different chirp rates. This higher responsiveness would have likely increased their chances of mating, and thus increased their probability of producing offspring. 


\subsubsection{Impact of Anthropogenic Noise on Adult Female Preference}

I found some support for my prediction that females exposed to anthropogenic noise should be more responsive than females raised without it. While this relationship was not significant for the preference function traits, there was a near significant response for my net vector score analysis. Specifically, I found that net vector score was influenced by a near-significant interaction between anthropogenic noise and chirp rate, where females reared with anthropogenic noise were less responsive to extreme chirp rates and instead preferred the population mean compared with females reared without anthropogenic noise. In previous studies, females exposed to anthropogenic noise during phonotaxis trials decreased orientation towards males in both crickets (T. oceanicus, (Gurule-Small and Tinghitella 2018; G. bimaculatus, Schmidt, Morrison, and Kunc 2014) and tree-frogs (Hyla chrysoscelis, Bee and Swanson 2007). The Gurule-Small and Tinghitella (2018) study focused on the effects of rearing environment, where females were raised either in silence, or in the presence of masking or non-masking traffic noise. Females raised in masking noise took significantly longer to move initially, and took $80 \%$ longer to locate a signalling male, then females raised in silence. Conversely, in five species of tree crickets (Oceanthus), anthropogenic noise did not affect females' response to male signals. A commonality in these studies however, is that they tested female phonotaxis while simultaneously playing anthropogenic noise. These studies are thus testing instantaneous responses to anthropogenic noise, rather than long-term exposure that could result in phenotypic behavioural variation (with the exception of Gurule-Small and Tinghitella 2018). Moreover, I included other human-related sounds in addition to traffic noise, which was modulated to represent real-world noise population and not a continuous 
broadcast. Not playing anthropogenic noise during my trials could partially account for the lack of significant difference in my groups, as females were still able to phono-locate a potential mate. I also did not test latency to move, so females reared with anthropogenic noise may have taken longer to respond; this is something to examine in future studies. As research into this area is still quite sparse, future work should more closely examine the relationship between adult behaviour determined through development and adult behavioural flexibility.

\subsubsection{Impact of Life History Trait Variation on Adult Female Preference}

In addition to social environment and anthropogenic noise, I also found an effect of female body size on preference and net vector scores. Larger females were both more responsive (preference function 1) and had higher net vector scores than smaller females (Tables 6.3, 6.4). Additionally, larger female preferentially had larger net vector scores in response to higher chirp rates, while smaller females preferred chirp rates around the population mean (Figure 6.6, C). Similar results have been found in several other species, where preference is related to female body size. For example, in the burying beetle (Nicrophorus orbicollis) female choice is related to females body size relative to male size, with larger females more likely to prefer larger males (Beeler et al. 2002).

Additionally, in another field cricket (Gryllus integer), females with a larger body mass were more selective of male acoustic signals than smaller females (Hedrick \& Kortet 2012). Body size is often used as a measure of condition (Jakob et al. 1996; Whitman 2008) and since sexual selection in females is often condition dependent, high quality females tend to show the strongest mate preference (reviewed in Cotton, Small, and Pomiankowski 2006). This phenomenon could be due to higher quality females being 
better able to tolerate the costs associated with mate searching and sampling, with larger females having more energy reserves (Whitman 2008). As larger females have more energy stores or higher fecundity - they can afford to be more discriminate in their mate choice, whereas females in poorer condition are more affected by the associated costs of mating and are thus less choosy (Whitman 2008).

My research adds to the growing number of studies suggesting that juvenile social environment plays a key role in not only development, but also in adult behaviour. The fact that females adjust their mating preference functions in response to male densities during rearing has important implications for both female and male fitness. Future work will hopefully elucidate how a female's response adapts throughout her entire lifetime, and if experiences during development result in set or plastic adult preference traits.

I also highlighted the important consequences that anthropogenic noise pollution may be having on female mate preference, and thus, male trait selection. Future studies should consider population differences that might exist between urban and rural populations, in case genetic predispositions are altering mate choice patters, similar to what Lampe et al. (2014) found in male grasshoppers. Another area of interest would be to examine if female mate choice is instantaneously adjusted to noise pollution in addition to long-term consequences. 


\section{Chapter 7: Can adult male field crickets adjust their signalling behaviour when presented with new social densities?}

\subsection{Abstract}

The ability of animals to accurately predict their future environmental conditions is an important component of developmental plasticity. One method animals use to ensure that their adult phenotype aligns with future conditions is social anticipatory plasticity where life history traits are driven by anticipated social dynamics. Using social anticipatory plasticity, juveniles can allocate resources during development to maximize their fitness at adulthood. For example, juvenile males reared in high densities can increase their growth rate, using their resultant larger adult body sizes to gain an advantage in their future environment, when they expect to experience high competition. One issue with plasticity studies is determining if behavioural responses at adulthood are the result of adult plastic behaviour (behavioral plasticity) or if their behaviour is constrained by their phenotype at maturity. To examine this relationship, I raised males while exposing them to acoustic social environments that represented either a high density of adult male acoustic signalling or social isolation (High or None). I then reciprocally manipulated their environments, such that half the males reared in each environment were transferred to the opposite environment at adulthood, while the other half remained in their original environment. I then quantified adult male mate signalling behaviour. I found no significant effect of developmental acoustic social environment, or adult acoustic social environment, on any fine scale signal parameters, time spent signalling, or amplitude. However, I found that chirp rate was influenced by a nearsignificant effect of the interaction between juvenile and adult acoustic social 
environments. Specifically, males exposed to adult signals, either during development or at adulthood, produced signals with higher chirp rates, while males that remained in social isolation signalled at lower, less energetically costly chirp rates. Additionally, juveniles that experienced acoustic signalling during rearing (High) developed faster, had a larger residual mass and a larger body size at adulthood than males reared in acoustic isolation (None). This is an important indirect effect because both body size and residual mass affected adult signalling. Larger males signalled more often and produced more energetically costly signals, at high amplitudes than smaller males, whereas males with lower adult residual mass signalled with faster pulse rates. These results raise several important questions concerning indirect effects and how researchers should go about determining how the interactions of developmental and behavioural plasticity shape the adult phenotype.

\subsection{Introduction}

Developmental plasticity, whether responsive or anticipatory, plays an important role in shaping adult phenotype (Whitman \& Agrawal 2009). Responsive plasticity occurs when an organism responds to immediate changes within the environment (West-Eberhard 2003; Whitman \& Agrawal 2009). Changes in food quality or availability can have detrimental or advantageous effects on current developmental trajectories leading to changes in life-history traits at adulthood. For example, Reifer et al., (2018) revealed that Jamaican field crickets (Gryllus assimilis) that had access to a diet that was rich in proteins but poor in carbohydrates had shorter development times and larger body sizes at adulthood than crickets with access to a diet that was rich in carbohydrates but poor in 
proteins. Alternatively, anticipatory plasticity occurs when phenotypic changes are produced in response to cues that indicate future environmental conditions (WestEberhard 2003; Whitman \& Agrawal 2009). Photoperiod and temperature are thought to be some of the most wide-spread examples of anticipatory plasticity as animals can determine oncoming seasonal changes based on variations in daylight and temperature. For example, shorter day lengths indicating a coming seasonal change from summer to fall result in faster larval development time in the blue butterfly (Polyommatus Icarus) (Leimar 1996). Furthermore, both temperature and photoperiod play a vital role in the maintenance of the circadian and circannual rhythms used by birds to time migration (Gwinner 1996).

When these environmental cues are related to population dynamics, the plastic response is termed socially cued anticipatory plasticity (Kasumovic \& Brooks 2011). Social cues can be transmitted in several forms but acoustic signals are fairly ubiquitous across taxa (Pollack 2017; Partan \& Marler 2005) and provide a unique opportunity for unintended receivers to garner information from them (Tomkins et al. 2004; McGregor \& Peake 2000). For example, in species that use acoustic communication, juveniles that eavesdrop on conspecific mate attraction signals have the potential to use these acoustic cues as indicators about the demographics and quality of males in their immediate environment and possible future population. Juveniles could therefore use the social cues contained in these mate attraction signals to alter their own developmental trajectories to enable them to better compete in their future environment (Kasumovic et al. 2011).

Socially cued anticipatory plasticity has been found in multiple species including zebra fish (Taeniopygia guttata, Tschirren, Rutstein, Postma, Mariette, \& Griffith, 2009), 
wolf spiders (Schizocosa rovneri, Rutledge, Miller, \& Uetz, 2010), and several cricket species (Gryllus integer DiRienzo et al. 2012; Teleogryllus commodus, Kasumovic et al. 2011; and Teleogryllus oceanicus, Bailey et al. 2010). Additionally, I found that juvenile fall field crickets (Gryllus pennsylvanicus) follow significantly different life history trajectories depending on the acoustic social environment they experience during development (Chapter 3). Furthermore, I found that the juvenile acoustic social environment significantly influenced aggression levels in losing male crickets (Chapter 4) and mate choice preference in female crickets (Chapter 6). Given that the acoustic social environment has the potential to alter both life history traits and adult behaviours, it should be considered when quantifying phenotypic and behavioural plasticity, their interactions, and their potential impacts on fitness.

Although the ability to accurately assess a future environment can have countless advantages, issues can arise if the environment changes after physical development has been completed. In cases like this, a mismatch between the current environment and the environment in which the individual developed tends to occur. Given this mismatch, the individual's fitness will depend on flexible traits that were not set during development. Environmental changes that require a behavioural response will thus rely on behavioural plasticity (or flexibility) (Duckworth 2010). Studies have shown that individual animals can display a wide range of behavioural responses. However, if these behaviours are partially reliant on traits constrained during development, individuals may not be able to adjust their behaviours to the same degree and as a result endure a cost to fitness due to a maladaptive phenotype (Mery \& Burns 2010; Duckworth 2010; Snell-Rood 2013; Taborsky 2017). As such, understanding how differences in the developmental acoustic 
social environments affect adult mating behaviour is the logical next step to be completed following my examination of how different developmental social environments affect life history traits and adult behaviours.

In my previous chapter on adult male signalling behaviour (Chapter 5) I found that the developmental acoustic social environment had indirect but not direct effects on adult male signalling. Given this, I wanted to determine if developmental traits that were the result of these indirect effects (adult body size) would constrain adult signalling in males, or if males could display flexible behaviour when exposed to different acoustic social environments at adulthood than the acoustic social environment that they developed in. To address this question, I raised males while exposing them to acoustic social environments that represented either a high density of adult male acoustic signalling or social isolation (High or None). I then reciprocally manipulated their adult environments, such that half the males reared in High were transferred to the None environment at adulthood, while half the males reared in the None were transferred to the High environment at adulthood. I predicted that if adult G. pennsylvanicus can display behavioural plasticity and adjust their behaviour in novel environments, then I would see a change in adult signalling behaviour between acoustic social environment switches. For example, males reared in social isolation exposed to the High density acoustic social environment at adulthood should be able to increase their signalling behaviour to contend with the now higher male competition. If, on the other hand, adult signalling behaviour is constrained by traits set during development, males would be unable to adjust their signalling behaviours and potentially display a miss-matched adult to environment phenotype as a result. 


\subsection{Methods}

\subsubsection{Study Species and Cricket Rearing}

To test how the developmental and adult acoustic social environment affects adult signalling behaviour I used laboratory-reared North American fall field crickets, $G$. pennsylvanicus. I randomly assigned each one-week old cricket (housed in individual containers) to one of two acoustic environment treatments and then placed each individual container into a larger communal treatment bin. The communal treatment bins were lined with acoustic foam to prevent sound contamination across treatments. Each communal treatment bin lid contained 4 speakers for playback of acoustic environment soundtracks. I used 2 of my original 6 acoustic soundtrack treatments (Chapters 3 - 6): 1 with the higher density of male acoustic signals (High), and 1 with no male acoustic signals (None). I did not include anthropogenic noise as a component of this experiment. Crickets were kept on a 14h:10h light:dark photoperiod at $28 \pm 2{ }^{\circ} \mathrm{C}$. Thrice weekly, each juvenile cricket had its food and water replenished and was checked for wing pad eclosion or adult eclosion. Chapter 2 provides a detailed description of how I produced these two different acoustic treatments, how I housed my individual crickets during rearing in their different acoustic treatments, how I quantified body size, and the other general protocols that I used for all data chapters.

Once crickets reached adult eclosion I weighed them and then randomly selected whether they would stay in their original acoustic social treatment or whether they would be switched to the opposite acoustic social treatment. I then housed these adult males in separate communal treatment bins away from the juveniles to eliminate acoustic interference within the juvenile acoustic environment (sensu Chapters 4-5). I housed a 
maximum of 6 adult males together at any one time to ensure that any potential extra signals produced by these experimental males were kept to a minimum. At 7 days postimaginal moult I quantified each male's acoustic mate attraction signalling behaviour.

\subsubsection{Adult Male Signalling Behaviour}

Male acoustic signalling behaviour was continuously monitored from day 7 to 14 post imaginal moult (7 days total) using the Electronic Acoustic Recording System (EARS-II; designed and developed by Cambridge Electronic Design, Cambridge, UK). Using the EARS II system, I recorded time spent signalling ( $\mathrm{min} / 24 \mathrm{~h}$ period) and 9 fine scale signalling parameters including: pulse duration (ms), interpulse duration (ms), pulse rate $(\mathrm{P} / \mathrm{min})$, pulses per chirp, chirp duration (ms), interchirp duration (ms), chirp rate (Ch/min), signal amplitude (Pa; converted to decibels using $20 \mathrm{X} \log$ (mean amp $(\mathrm{Pa}) / 0.00002 \mathrm{~dB})$ ), and carrier frequency $(\mathrm{Hz})$. Acoustic files were analyzed to produce a summary of mean signalling parameters using Spike2 v6.12 (Cambridge Electronic Design, Cambridge, UK). After their signals were recorded, males were removed from the EARS II containers, weighed and then euthanized via freezing. Male signalling behaviour was analyzed using adults that eclosed between March 21, 2017 and October 10, 2017. Please refer to Chapter 5 for more detailed information about the EARS II system and male acoustic signalling parameters.

\subsubsection{Statistics}

All statistical analyses were completed using JMP statistical software (version 11.0.0 SAS Institute). To determine adult residual mass and body size at adult eclosion, I combined body size measurements using a principle component analysis (PCA) and 
extracted orthogonal vectors from pronotum length, pronotum width, and head width. The first principal component (PC1 size) explained $95 \%$ of the variation in adult male body size $($ Eigenvalue $=2.9)$; all size measures loaded equally on PC1 size (eigenvectors ranged from 0.57 to 0.58$)$. I then regressed body size (PC1 size) against body weight at adult eclosion to determine residual mass.

Following the statistical protocols set out in Chapter 3 and 4, I used linear models (LMs) to examine the effects of the juvenile acoustic social environment the males experienced on their development time, adult body size (PC1 size), and adult residual mass. Although I found no effect of juvenile acoustic social environment on signalling in Chapter 5, I repeated these statistical analyses because there was an indirect effect of juvenile environment on development time, which in turn affected signalling behaviour. For these LMs the dependent variables were days from hatching to Wing pad eclosion, days from Wing pad eclosion to adult eclosion, days from Hatching to Adult eclosion (total development time), adult body size (PC1 Size), and adult residual mass. The independent variable in each model was juvenile signalling density (High or None). Because I ran my experiment over a 6-month period, I also included season of hatching as a covariate to control for possible block effects.

Following the protocol and rationale I outlined in Chapter 5, I performed a PCA to extract orthogonal vectors from eight of the fine-scale acoustic signalling characteristics (pulse duration, interpulse duration, pulse rate, pulses per chirp, chirp duration, interchirp duration, chirp rate, and carrier frequency. I excluded time spent signalling and amplitude from this PCA because I had made explicit predictions about how a male should signal more often and louder if he was reared in an isolated (None) environment with no adult 
signalling and then exposed to an environment with signalling (High) at adulthood. For interpretation of the PCA, I performed varimax rotation on the first 3 principle components. The first three rotated principle components were selected (signal factor 1, 2 and 3) as they had eigen values above 1 (Kaiser 1964) and satisfied the scree test for factor rotation conditions (Cattell 1966). All three signal factors were indicative of signalling effort, with higher scoring males producing more energetically costly signals. Signal factor $1($ Eigenvalue $=3.87)$ explained $30 \%$ of the signalling variation and was indicative of longer pulse durations and shorter interpulse durations, and high scoring males thus signalled with faster pulse rates. Signal factor $2($ Eigenvalue $=2.26)$ explained $20 \%$ of the signalling variation and higher scoring males had faster chirp rates because of shorter interchirp durations. Signal factor 3 (Eigenvalue $=1.29$ ) explained $11 \%$ of the variation and was indicative of longer chirp durations (Table 7.1). 
Table 7.1. PCA factor loading values for signal factor 1,2 and 3 of 8 mean daily fine scale signalling compoents after varimax roation. A negative sign (-) on the loading indicates that higher levels of that factor are negatively associated with that signalling parameter. Loadings above 0.600 are shown in bold.

\begin{tabular}{lrrr}
\hline Fine Scale Signal Component & Signal factor 1 & Signal factor 2 & Signal Factor 3 \\
\hline Eigenvalue & 3.87 & 2.56 & 1.29 \\
Percent & 29.77 & 19.85 & 10.62 \\
Cumulative Percent & 29.77 & 49.61 & 60.24 \\
Pulse Duration (ms) & $\mathbf{0 . 7 5 0}$ & -0.085 & 0.332 \\
Interpulse Duration (ms) & $\mathbf{- 0 . 9 8 0}$ & -0.009 & -0.136 \\
Pulses Per Chirp & 0.420 & 0.153 & 0.546 \\
Chirp Duration (ms) & -0.012 & 0.071 & $\mathbf{0 . 6 3 0}$ \\
Interchirp Duration (ms) & 0.153 & $\mathbf{- 0 . 7 1 2}$ & -0.207 \\
Pulse Rate (\#Pulses/sec) & $\mathbf{0 . 9 3 3}$ & 0.039 & -0.054 \\
Chirp Rate (Chirps/min) & 0.193 & $\mathbf{0 . 9 2 1}$ & 0.124 \\
Carrier Frequency (Hz) & -0.223 & -0.176 & -0.265 \\
\hline
\end{tabular}


I used linear models (LMs) to examine the effects of the acoustic social environment experienced during development and then at adulthood on adult males' long-distance mate attraction signalling behaviour. The dependent variables I used were signal factor 1 (pulse rate), signal factor 2 (chirp rate), single factor 3 (chirp duration), time spent signalling, and amplitude $(\mathrm{dB})$. The independent variables were juvenile signalling density (High or None), adult signalling density (High or None), adult body size (PC1 Size) and their interactions. I included residual mass at adult eclosion, and season as covariates. I tested LMs for violations of normality by examining the distribution of residual diagnostic plots (residuals vs fitted values and Q-Q plots); I also tested LMs for violations of homoscedasticity by looking for dependency between the residuals and the fitted values. Normality and homoscedasticity assumptions were not violated. P-values were corrected for using false discovery rate FDR $\mathrm{B}_{\mathrm{Y}}$ method (Benjamini \& Yekutieli 2001).

\subsection{Results}

I analyzed the signalling behaviour of 124 adult males. Of these, 4 males did not signal, and 2 males were outliers so I removed them from the analysis, resulting in a final count of 118 (Juvenile:Adult density: High:High = 28; High:None = 34; None:None = 28; None:High $=28$ ).

\subsubsection{Adult Male Signalling Behaviour}

Adult male signalling, specfically, time spent signalling, amplitude, signal factor 1 (pulse rate), signal factor 2 (chirp rates), and signal factor 3 (chirp durations) were not significantly influenced by either the juvenile acoustic social environment, the adult 
acoustic social environment, or the interaction between them (Table 7.2). I did, however, find a near significant effect of the intereaction between juvenile and adult social acoustic density on signal factor $2(P=0.08$, Table 7.2, Figure 7.1). Males that were reared in the High density acoustic social environment during development (High:High and High:None) produced signals with fast chirp rates as adults, regardless of the adult environment they entered. Additionally, males reared in social isoaltion (None) and then switched to the High density acoustic social environment at adulthood also produeced signals with faster chirp rates. Conversely, males that remained in social isolation throughout their developmental and adult life stages (None:None) signalled at slower chirp rates (Figure 7.1).

Time spent signalling, and amplitude were significantly affected by adult body size (PC1 Size)(Table 7.2). Larger males also spent more time signalling $(P=0.012$, Table 7.2, Figure 7.2 B), and signalled at higher amplitudes $(P=<0.0001$, Table 7.2, Figure 7.2 C). Signal factor 1 (pulse rate) also showed a near significant affect of adult body size (Table 7.2), wiht larger males tending to have faster pulse rates $(P=0.05$, Table 7.1, Figure 7.2 A). Additionally, males with lower residual mass signalled with faster pulse rates $(P=0.0111$, Table 7.2, Figure 7.3). 
Table 7.2. Results of the linear models examining effects of juvenile and adult acoustic treatment, adult body size, residual mass at adulthood and before the signalling trial, and season on signal factors 1, 2 and 3, time spent signalling, and amplitude. Significant effects are bolded for both models and model effects. FDR $\mathrm{BY}_{\mathrm{BY}}$ corrected level of significance is $P=0.018(\mathrm{~N}=118)$. Parameter estimates are included in Appendix 6, Table A6.1.

\begin{tabular}{|c|c|c|c|c|c|c|c|}
\hline Model & $\mathbf{R}^{2}{ }_{\text {adj }}$ & F model & $P_{\text {model }}$ & Model Effect & DF & $\mathbf{F}$ & $\mathbf{P}$ \\
\hline \multirow[t]{9}{*}{ Signal Factor 1} & 0.21 & 3.89 & $<\mathbf{0 . 0 0 0 1}$ & Season & 3 & 7.41 & 0.0001 \\
\hline & & & & Juvenile Signalling Density & 1 & 1.34 & 0.25 \\
\hline & & & & Adult Signalling Density & 1 & 1.97 & 0.16 \\
\hline & & & & Juvenile Signalling Density*Adult Signalling Density & 1 & 0.32 & 0.57 \\
\hline & & & & PC1(Size) & 1 & 4.03 & 0.05 \\
\hline & & & & Juvenile Signalling Density*PC1(Size) & 1 & 0.33 & 0.57 \\
\hline & & & & Adult Signalling Density*PC1(Size) & 1 & 1.69 & 0.20 \\
\hline & & & & Juvenile Signalling Density*Adult Signalling Density*PC1(Size) & 1 & 3.25 & 0.07 \\
\hline & & & & Residuals Mass at Adult Eclosion (mg) & 1 & 6.68 & 0.0111 \\
\hline \multirow[t]{9}{*}{ Signal Factor 2} & 0.14 & 2.79 & 0.0031 & Season & 3 & 5.52 & 0.0014 \\
\hline & & & & Juvenile Signalling Density & 1 & 0.01 & 0.90 \\
\hline & & & & Adult Signalling Density & 1 & 0.90 & 0.34 \\
\hline & & & & Juvenile Signalling Density*Adult Signalling Density & 1 & 3.17 & 0.08 \\
\hline & & & & PC1(Size) & 1 & 1.65 & 0.20 \\
\hline & & & & Juvenile Signalling Density*PC1(Size) & 1 & 0.82 & 0.37 \\
\hline & & & & Adult Signalling Density*PC1(Size) & 1 & 0.08 & 0.78 \\
\hline & & & & Juvenile Signalling Density*Adult Signalling Density*PC1(Size) & 1 & 1.23 & 0.27 \\
\hline & & & & Residuals Mass at Adult Eclosion (mg) & 1 & 0.28 & 0.60 \\
\hline \multirow[t]{2}{*}{ Signal Factor 3} & 0.02 & 1.28 & 0.25 & Season & 3 & 0.27 & 0.85 \\
\hline & & & & Juvenile Signalling Density & 1 & 2.04 & 0.16 \\
\hline
\end{tabular}


Adult Signalling Density

$\begin{array}{ll}0.25 & 0.61\end{array}$

Juvenile Signalling Density*Adult Signalling Density

$\begin{array}{lll}1 & 0.07 & 0.79\end{array}$

PC1(Size)

Juvenile Signalling Density*PC1(Size)

$2.11 \quad 0.15$

Adult Signalling Density*PC1(Size)

$0.56 \quad 0.46$

Juvenile Signalling Density*Adult Signalling Density*PC1(Size)

$\begin{array}{ll}0.00 & 0.97\end{array}$

Residuals Mass at Adult Eclosion (mg)

\begin{tabular}{|c|c|c|c|c|c|c|c|}
\hline \multirow{8}{*}{$\begin{array}{l}\text { Time Spent } \\
\text { Signalling }\end{array}$} & \multirow{8}{*}{0.07} & \multirow{8}{*}{1.82} & \multirow{8}{*}{0.06} & Residuals Mass at Adult Eclosion (mg) & 1 & 0.16 & 0.69 \\
\hline & & & & Season & 3 & 0.28 & 0.84 \\
\hline & & & & Juvenile Signalling Density & 1 & 0.70 & 0.41 \\
\hline & & & & Adult Signalling Density & 1 & 0.08 & 0.78 \\
\hline & & & & Juvenile Signalling Density*Adult Signalling Density & 1 & 0.01 & 0.91 \\
\hline & & & & PC1(Size) & 1 & 6.53 & 0.012 \\
\hline & & & & Juvenile Signalling Density*PC1(Size) & 1 & 0.49 & 0.49 \\
\hline & & & & Adult Signalling Density*PC1(Size) & 1 & 0.03 & 0.87 \\
\hline \multirow{5}{*}{ Amplitude (db) } & \multirow[t]{5}{*}{0.24} & \multirow[t]{5}{*}{4.48} & \multirow[t]{5}{*}{$<0.0001$} & Season & 3 & 0.93 & 0.43 \\
\hline & & & & Juvenile Signalling Density & 1 & 1.48 & 0.23 \\
\hline & & & & Adult Signalling Density & 1 & 0.59 & 0.44 \\
\hline & & & & Juvenile Signalling Density*Adult Signalling Density & 1 & 0.40 & 0.53 \\
\hline & & & & PC1(Size) & $\mathbf{1}$ & 23.34 & $<0.0001$ \\
\hline
\end{tabular}




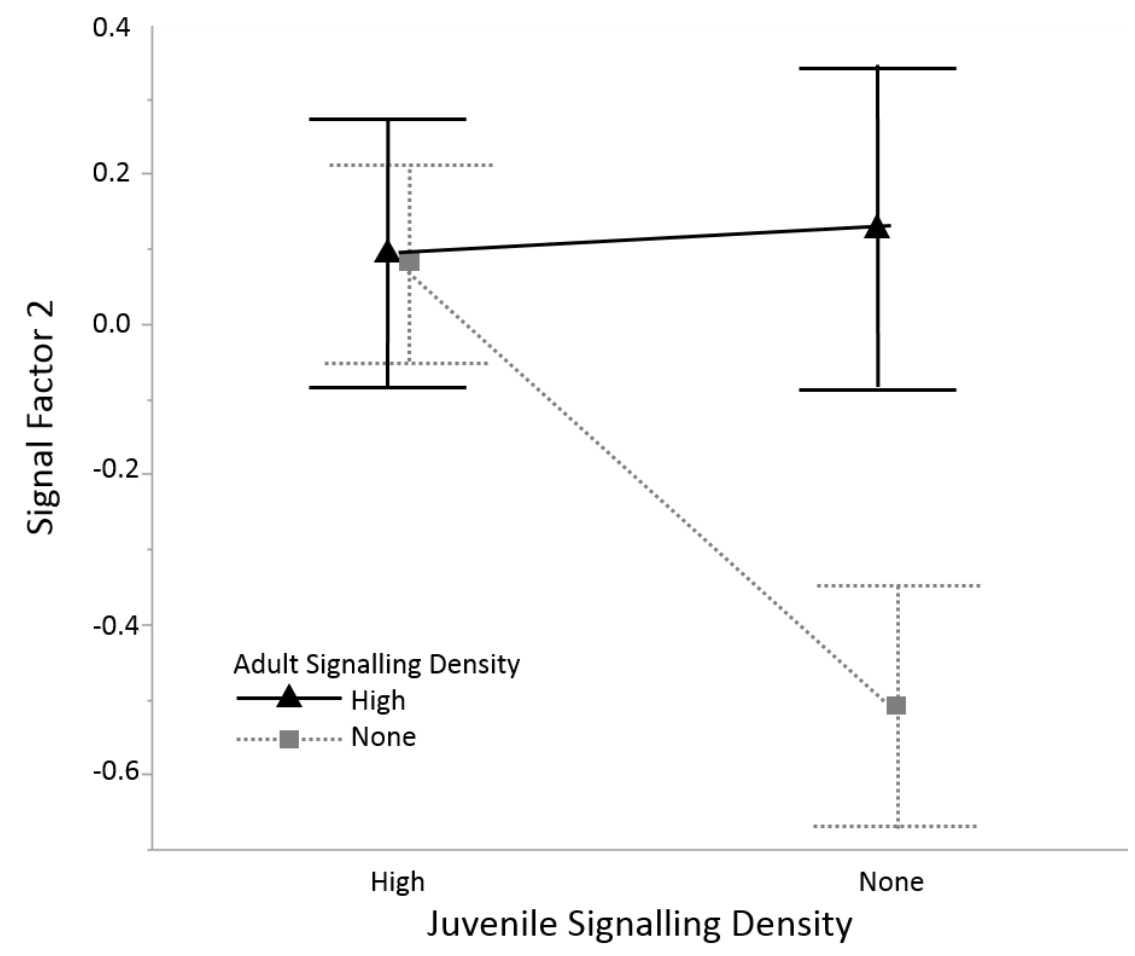

Figure 7.1. How the interaction between juvenile acoustic signalling density and adult acoustic signalling density affects signal factor 2 . Bars are standard errors. 

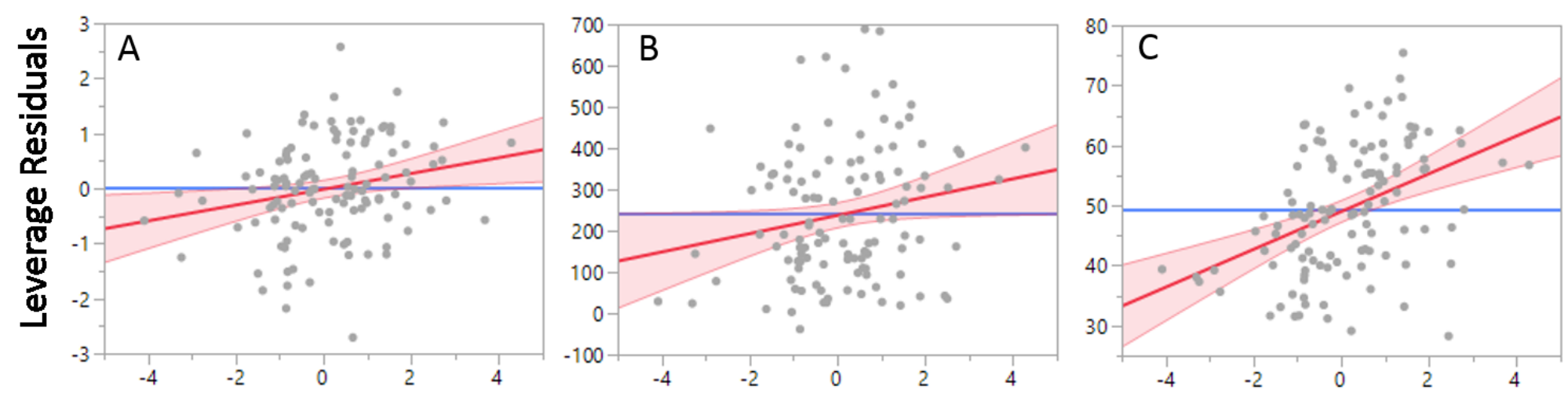

Adult Body Size (PC1 Size)

Figure 7.2. Leverage residual plot showing the effects of adult body size (PC1 size) on A) signal factor 1 (near significant), B) time spent signalling (min), and C) amplitude (db). Solid line indicates the relationship between body size and signalling component after all other variables in the model has been accounted for; the dashed lines represent $95 \%$ confidence intervals. 


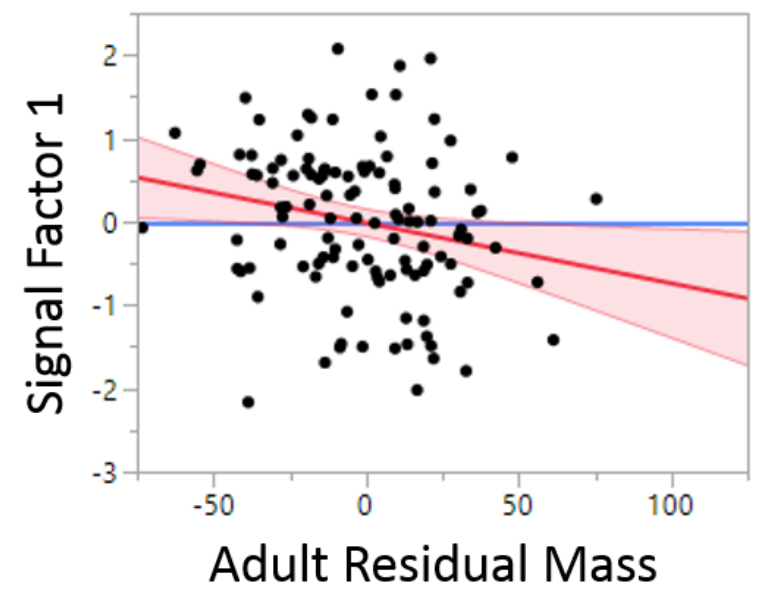

Figure 7.3. Leverage residual plot showing the effects of adult residual mass on signal factor 1 . Solid line indicates the relationship between body size and signalling component after all other variables in the model has been accounted for; the dashed lines represent $95 \%$ confidence intervals. 


\subsubsection{Impact of Acoustic Treatments on Life History Traits}

Because the males in this chapter are different from the males I used in previous chapters, I repeated the statistical analyses conducted in Chapter 3 to determine how acoustic treatment during development influenced developmental life-history traits. Male development time was significantly affected by the acoustic treatment (Table 7.3). Males raised in the High density acoustic social environment reached adult eclosion approximately 10 days sooner than males raised in the Low density acoustic social environment $(P=0.0003$, High $\bar{x} \pm S E=67.4 \pm 1.8$, None $\bar{x} \pm S E=76.9 \pm 1.4)$ (Table 7.3, Figure 7.4). Additionally, both male body size (PC1 size) and residual mass at adulthood were significantly affected by the juvenile acoustic social environment's signalling density treatment (Table 7.5). Specifically, males raised in the High density acoustic social environment were larger $(P=<0.0001$, High $\bar{x} \pm S E=0.58 \pm 0.23$, None $\bar{x} \pm S E=-0.64 \pm 0.22$, Figure 7.5) and had higher residual mas $(P=<0.0001$, High $\bar{x} \pm$ $S E=10.10 \pm 5.38$, None $\bar{x} \pm S E=-11.12 \pm 4.18$, Figure 7.4) at adult eclosion than males raised in social acoustic isolation (None). 
Table 7.3. Results of the linear models examining effects of acoustic treatment and season on development time, adult body size (PC1 size), residual mass at adulthood. Significant effects are bolded for both models and model effects. FDR $\mathrm{BY}_{\mathrm{C}}$ corrected level of significance is $P=0.033(\mathrm{~N}=124)$. Parameter estimates are included in Appendix 6, Table A6.2.

\begin{tabular}{|c|c|c|c|c|c|c|c|}
\hline Model & $\mathbf{R}_{\mathbf{a d j}}^{2}$ & $\mathbf{F}_{\text {model }}$ & $\mathbf{P}_{\text {model }}$ & Model Effect & DF & $\mathbf{F}$ & $\mathbf{P}$ \\
\hline \multirow[t]{2}{*}{ Hatching to Wing pad } & 0.42 & 22.92 & $<0.0001$ & Season & 3 & 29.97 & $<0.0001$ \\
\hline & & & & Signalling Density & 1 & 0.16 & 0.69 \\
\hline \multirow[t]{2}{*}{ Wing pad to Adult Eclosion } & 0.48 & 29.70 & $<0.0001$ & Season & 3 & 21.16 & $<0.0001$ \\
\hline & & & & Signalling Density & 1 & 30.11 & $<0.0001$ \\
\hline \multirow[t]{2}{*}{ Hatching to Adult Eclosion } & 0.62 & 50.23 & $<0.0001$ & Season & 3 & $\mathbf{5 7 . 5 2}$ & $<0.0001$ \\
\hline & & & & Signalling Density & 1 & 14.12 & 0.0003 \\
\hline \multirow[t]{2}{*}{ PC1 (Size) } & 0.16 & 6.80 & $<0.0001$ & Season & 3 & 2.71 & 0.05 \\
\hline & & & & Signalling Density & 1 & 26.12 & $<0.0001$ \\
\hline \multirow[t]{2}{*}{ Residual Mass } & 0.10 & 4.23 & 0.0031 & Season & 3 & 0.47 & 0.71 \\
\hline & & & & Signalling Density & 1 & 15.48 & 0.0001 \\
\hline
\end{tabular}




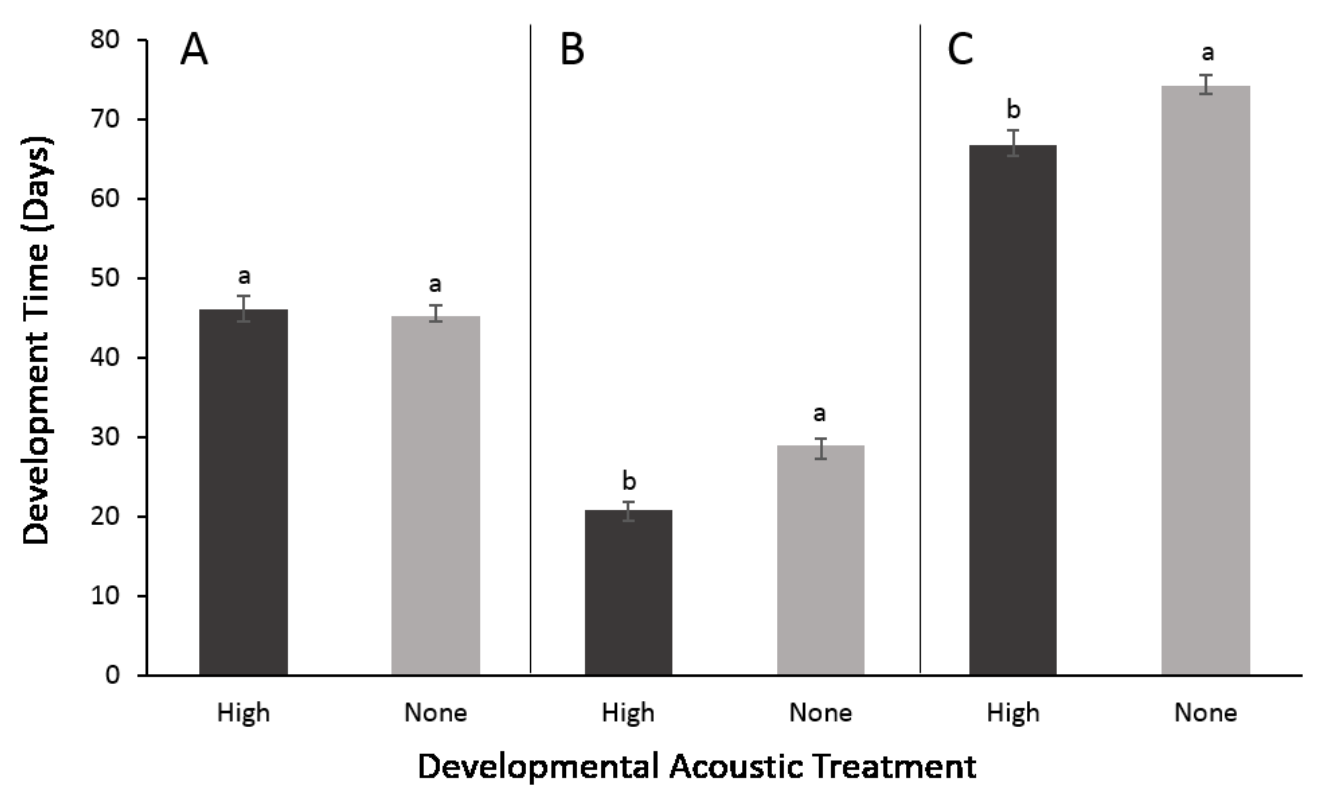

Figure 7.4. Effect of developmental acoustic treatment (signalling density) on development time (days) for the different life stages: (A) Hatching to Wing pad, (B) Wing pad to adult eclosion, and (C) Total Development Time. Error bars are standard errors and lowercase letters denote significant differences between acoustic treatment groups for each life stage. 


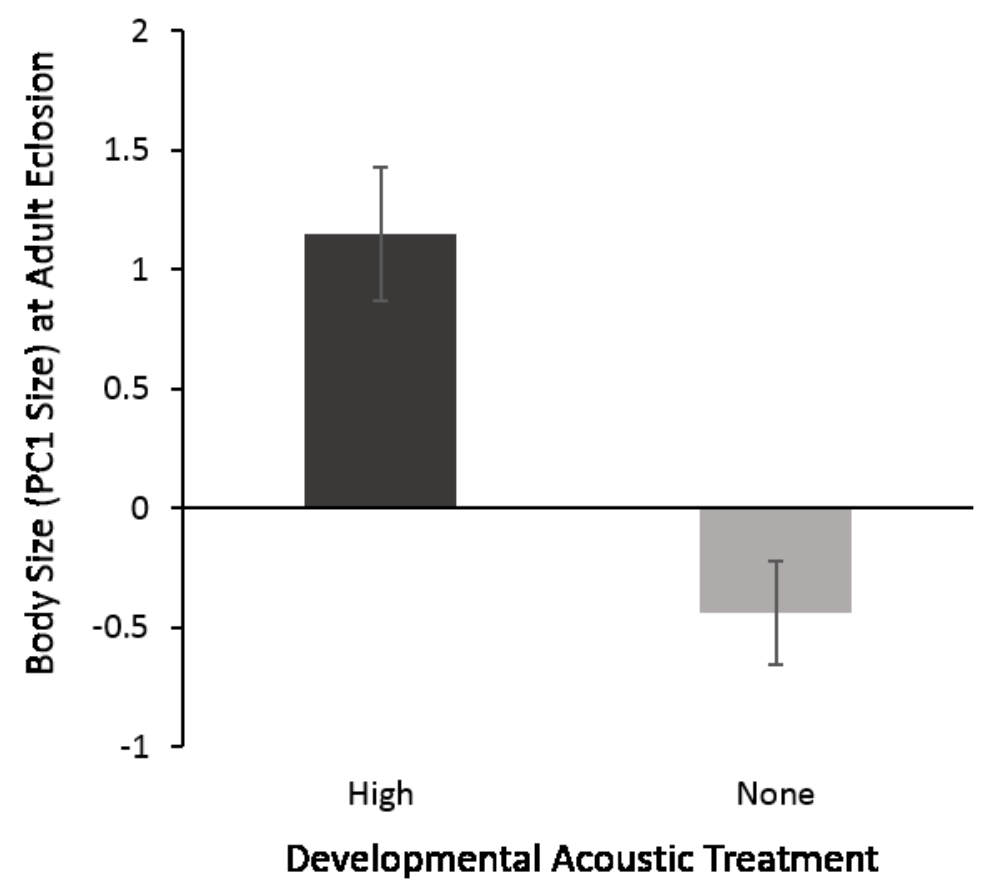

Figure 7.5. How the acoustic social environment during development influences male body size (PC1 size) at adult eclosion. Bars represent the average PC1 size and error bars are standard errors; letters denote significant differences. 


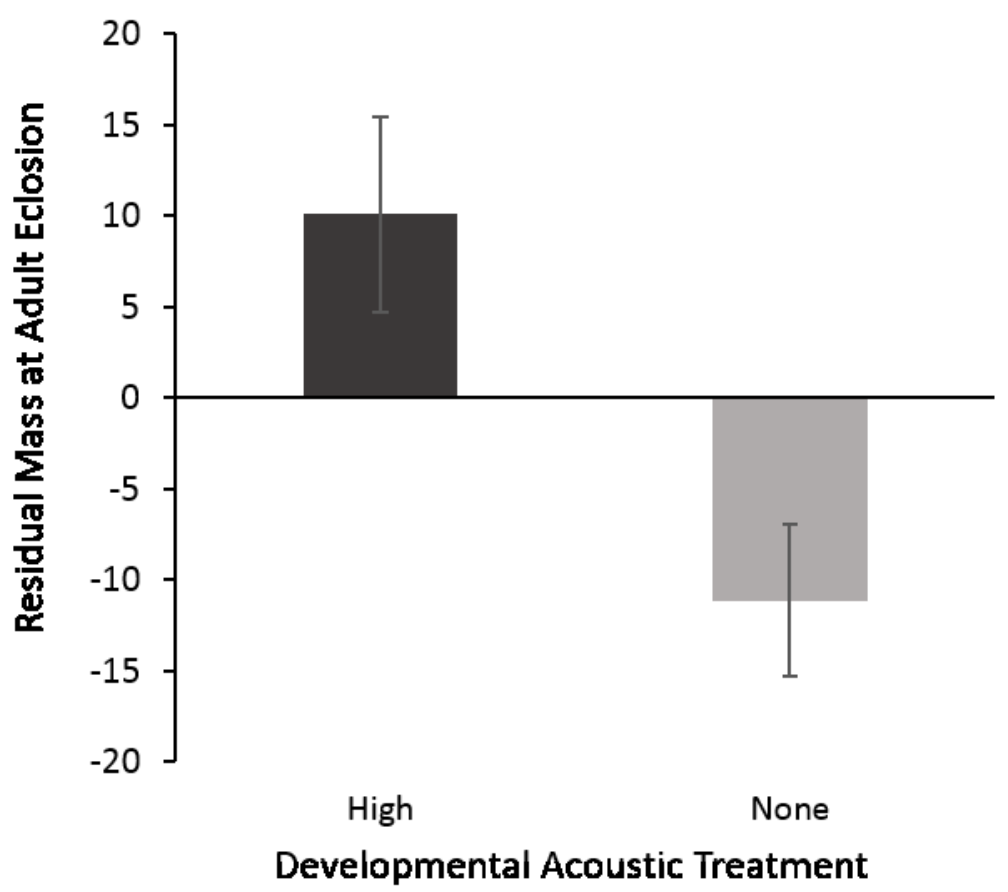

Figure 7.6. How the acoustic social environment during development influences male residual mass at adult eclosion. Bars represent the average PC1 size and error bars are standard errors; letters denote significant differences. 


\subsection{Discussion}

I designed this experiment to determine if changes in the acoustic social environment between juvenile and adult life stages would result in observable differences in adult behaviour. Specifically, I was interested in testing whether adult male signalling behaviour in Gryllus pennsylvanicus is more dependent on their juvenile acoustic social experience, or if their signalling behaviour is plastic and can therefore adjust to changes in the acoustic social environment experienced in adulthood. Similar to Chapter 5, however, I found no significant direct effects of acoustic social environment on adult male signalling. Specifically, neither juvenile, adult, or a change in the acoustic social environment between juvenile and adult life stages affected fine scale signalling parameters, time spent signalling, or amplitude. As discussed previously (Chapter 5) this may be partially the result of signalling behaviour being an energetically costly activity, and as such it is highly dependent on condition. For example, males reared on different diets often have different signalling behaviour, with those males raised on higher quality foods signalling more and producing more attractive (energetically expensive) signals (Reifer et al. 2018; Harrison et al. 2017; Kasumovic, et al. 2012b). As males used in my study were all given the same high quality diet throughout development and adulthood, differences due to condition may not have been apparent and the acoustic social environment alone may not have had enough of an effect to cause changes in signalling behaviour.

Interestingly, there was some evidence of a possible direct effect of the acoustic social environment influencing adult male signalling behaviour. I found a non-significant trend for an interaction between the juvenile and adult social acoustic signalling 
environments on chirp rate (signal factor 2). Males that experienced acoustic isolation during development (None:None) tended to produced signals with slower chirp rates. Conversely, males that experienced acoustic signalling during development (High:High and High:None) signalled with faster, more energetically expensive chirp rates as adults, regardless of the acoustic social environment they experienced in adulthood. Thus, even when males reared in the High acoustic social environment had no acoustic competitors at adulthood, they still signalled with faster chirp rates. On its own this finding suggests that the juvenile environmental experience may constrain males from behaviourally responding to changes in their environment at adulthood. Fascinatingly though, males that developed in acoustic isolation but who then experienced male acoustic signals during adulthood (None:High) produced energetically costly signals with chirp rates similar to those males that were exposed to acoustic mate attraction signals throughout development. Together these findings suggest that males are not constrained from behaviourally responding to changes in their environment at adulthood. Instead, regardless of the timing of exposure, higher social densities instigate males to elevate their chirp rates. Plasticity therefore seems to be acting on male chirp rate at both the developmental and behavioural levels.

This trend is fascinating as it aligns very well with my female results. Female field crickets prefer males that signal at faster chirp rates (Chapter 6, Simmons, 1988; Wagner \& Reiser, 2000; Wagner, 1996) that are energetically costly to produce (Pollack \& Hoy 1981; Tolle \& Wagner 2010). This is especially true in G. pennsylvanicus, where females exhibit directional preference functions that target males that signal at high chirp rates (Chapter 6). Importantly, my key results in Chapter 6 revealed that females that 
developed in High density acoustic social environments were choosier and less responsive than females that developed in acoustic isolation. Here my results show that when males are at risk of competition, regardless of the life stage (e.g., High:High, High:None, None:High) they elevate their chirp rates to align with elevated female mating preferences. However, when males are not at risk of competition (None:None) they signal at lower less energetically demanding chirp rates. Thus, it appears that the developmental and behavioural plasticity that is acting on male chirp rate has strong fitness consequences.

Similar to the findings described in Chapter 5, I found that male body size at adult eclosion significantly affected signalling behaviour. Larger males produced more energetically costly signals (near significant), signalled more often, and at a higher amplitude than smaller males. This is consistent with previous studies in crickets where larger produce more attractive signals (Harrison et al. 2013; Bertram \& Rook 2012; Reifer et al. 2018). Animals with higher metabolic activity and with larger body sizes are often in better condition (Gillooly \& Ophir 2010; Jakob et al. 1996). Long distance mate attraction signals in crickets are energetically expensive to produce (Hoback \& Wagner 1997; Wagner \& Wyatt Hoback 1999) and males in better condition often produce more attractive signals (Judge et al. 2008; Harrison et al. 2013; Hannes Scheuber et al. 2003). Larger adult males, therefore, may have been able to better allocate developmental resources towards body size over other traits, resulting in more attractive signalling qualities.

The juvenile acoustic social environment appears to have an indirect effect on adult male signalling. Males reared in the High density acoustic social environment were 
significantly larger (body size) and heavier (residual mass) than males raised in acoustic social isolation (None). Since adult male signalling was significantly impacted by adult male body size, the developmental acoustic social environment was indirectly responsible for signalling behaviour. Adult male body size in crickets is set at adulthood (Whitman 2008), as is wing-size and the stridulating apparatus, although changes due to wear can occur with age (Judge 2008). Modulations to signalling after development must therefore be the result of changes in physical effort. Here, however, signalling effort was not found to have changed between treatment groups. There are several possible explanations for this finding. Foremost, it may have been due to males not receiving acoustic play-back during the recording of signalling behaviour. Due to the nature of the EARS II system, broadcasting cricket chirps to males while recording their chirping behaviour confounds our ability to quantify the male signalling behaviour. As such, males may not have been stimulated to instantaneously alter their signalling behaviour in response to conspecific signals, similar to noise response in male tree crickets (Oecanthus pellucens, Orci et al. 2016). Another possibility is that smaller males require a much more energetically subsidized diet than the one they were provided in order to compensate for energy requirements of more attractive signalling. In another field cricket (Gryllus lineaticeps) adult male signalling effort was found to be nutrition dependent, where higher-quality foods resulted in more time signalling and higher chirp rates (Wagner \& Wyatt Hoback 1999). Thus, size differences resulting from different developmental acoustic social treatments may have constrained flexibility in adult male signalling behaviour. Males reared in the High density acoustic social environment may have anticipated either/or both a) higher general population densities, with multiple mating opportunities, 
potentially leading to a faster development time to ensure ample occasions to mate, and/or b) greater intraspecific competition, potentially leading to resource allocation during development towards larger adult body sizes, ensuring an advantage in both mating and potential aggressive contests. Males reared in the None acoustic social environment may have anticipated the opposite adult density scenarios due to a lack of social input and thus allocated resources away from development time towards sperm number, building signalling muscles, or reserves to fuel signalling muscles, as these males should have to signal more often or louder to attract elusive females at adulthood. As my near significant findings revealed males from the social isolation acoustic treatment (None) were, in fact, able to adjust their chirp rates when faced with a more populated adult cohort (High), suggesting that their small size did not constrain adult behavioural plasticity.

Here I show that while the juvenile environment can affect life history traits and behaviours, adult male G. pennsylvanicus appear to be able to adjust their behaviour to accommodate a different social environment than the one they occupied as a juvenile. This ability to dynamically adjust their signalling behaviour is important, given the fitness consequences associated with signalling at preferred chirp rates. Because developmental plasticity results in specific adult phenotypes, including behavioural phenotypes, future studies should consider the impact of developmental constraints on adult behaviour before quantifying behavioural flexibility during adulthood. 


\section{Chapter 8: General Conclusions}

\subsection{Influence of Juvenile Acoustic Social Environment on Life History Traits}

In this dissertation I have shown that the acoustic social environment experienced during development influences variation in life-history traits of both male and female Gryllus pennsylvanicus field crickets. I used acoustic playbacks to expose developing male and female fall field crickets to acoustic mate attraction signals indicative of different social densities. Both males and females exposed to High signalling densities developed faster than males and females exposed to either Low signalling densities or no signals indicative of acoustic social isolation (None) (Chapter 3). Furthermore, the effects of the developmental acoustic social environment were more apparent in the time between wing pad eclosion and adult eclosion. This delayed effect could potentially be attributed to tympanic membrane development. While the hearing organs have their basic form at hatching, full development does not occur until around the $5^{\text {th }}$ or $6^{\text {th }}$ instar - so while precocious hearing is possible, hearing would be more advanced after the development of wing pads (Rössler 1992; Ball \& Young 1974). For development time, this phenomenon was observed more in males than females, suggesting that the female hearing organ may develop slightly earlier, possibly owing to the importance of listening in phonotaxis and mate location. Overall, these findings suggest that juvenile crickets allocate resources towards development time differently depending on the social dynamics they expect to face at adult eclosion.

Adult male body size was not directly influenced by the acoustic social environment during development. In females, however, those raised in the High density acoustic social environment had a larger adult body size compared to females raised in 
the Low density acoustic social environment (Chapter 3). Further, I found that body size was indirectly influenced by the acoustic social environment during development. The acoustic social environment influenced development time, and development time in turn, influenced adult body size (Chapter 3). Specifically, faster development resulted in larger individuals (males and females). These results were unexpected for males as they were contrary to what has been found in other studies where juvenile males exposed to adult male signalling took longer to develop and were also larger upon adult eclosion (Kasumovic et al. 2011; Bailey et al. 2010; DiRienzo et al. 2012). Juvenile males in my treatments may have anticipated an adult population that would have high numbers of both males and females, causing males to develop faster in order to take advantage of mating opportunities while simultaneously having larger bodies to contend with possible male-male competition.

\subsection{Influence of Juvenile Acoustic Social Environment on Adult Behaviour}

My findings revealed that the developmental acoustic social environment experienced by juvenile crickets influences adult behaviour of both males and females. The effect on aggression was most apparent in the fight losers, as losers that were raised in acoustic social isolation were more aggressive than losers reared in the presence of conspecific adult signals (Chapter 4). Furthermore, all of my experimental (focal) males, regardless of the acoustic social environment they were reared in, were more aggressive and more likely to win fights than their opponents. This result is likely attributed to the fact that my focal males were all raised in physical isolation, while the opponents were reared in groups. Physical isolation results in a lack of social information during develoment, which may cause males to incorrectly assess the costs of aggressive behaviour, leading to 
hightened agnostic responses as adults. These findings are in agreement with other studies that have also found that social isolation during development leads to increased levels of adult aggression (DiRienzo et al. 2012; Nagamoto et al. 2005).

Conversely, indirect effects of the developmetal acoustic social environment suggest that exposure to conspecific signalling as juveniles may also lead to increased aggression. Juvenile males exposed to adult male signalling developed faster, and faster development resulted in larger body size. Larger adult males are both more aggressive and win more fights than smaller males. This not only suggests that body size may be a more predictive factor than the acoustic social experience during development for winning fights and aggressive behaviour overall, but that body size can also be affected by acoustic social experience. It is vital, therefore, that previous life history be considered when studying the effects of social environment on male aggression.

I found no significant direct effects of developmental acoustic social environment on adult male signalling behaviour, as there was no difference in fine scale signalling parameters, time spent signalling, or amplitude between groups (Chapter 5). Similar to Chapter 4, I found indirect effects of juvenile acoustic social environment related to adult body size. Larger males signalled more attractively than smaller males, as they produced more energetically costly signals, signaled at higher amplitudes, and spent more time signalling. This is important, as males raised in the High density acoustic social environment developed faster, and faster development led to larger body sizes. Thus, indirectly, males raised in the High density environment had more attractive signals than those raised in the Low and None environments. As male mate attraction signals in crickets are highly correlated with adult body size (Bertram \& Rook 2012; Harrison et al. 
2013; Judge et al. 2008; Reifer et al. 2018), constraints on signalling ability may already be in place at adult eclosion when other variables (such as food availability) are held constant. However, my results from Chapter 7 suggest that regardless of the timing of exposure, higher social densities instigate males to produce more energetically costly signals (specifically chirp rates). Thus, while some developmental constraints may exist limiting the plasticity of certain signalling parameters, such as amplitude), males may be able to behaviourally adjust other parameters, such as chirp rate, when faced with new social information at adulthood.

In contrast to males, adult behaviour in females was more directly affected by the developmental acoustic social environment. Social isolation during development (None environment) resulted in females that were more responsive across all artificial signals compared to males reared in the Low or High acoustic social environments. This finding is consistent with previous studies that show that social experience during development and at adulthood (physical or acoustic) results in females that are less responsive and more choosy (Bailey \& Zuk 2008; Judge 2010; Bailey \& Zuk 2009). These findings suggest the females from a high density environment will be choosier and more discriminating adults in terms of chirp rate preference. This could potentially result in females mating with a smaller range of males, resulting in stronger directional selection for male chirp rate. Females with little to no social input during development however would be less discriminating and thus potentially willing to mate with a wider male population, resulting in more relaxed selection on male chirp rate.

Additionally, I found that body size also influenced female responsiveness, where larger females were more responsive than smaller females. Larger females also preferred 
a larger range of artificial signals, while smaller females showed preference towards signals at the population mean. Thus, both juvenile acoustic social experience and adult body size are important when considering how female preference impacts selection for male traits. Interestingly, if we consider indirect effects, the acoustic social environment can be seen to act on female preference in opposite ways. Development in the High density environment results in females with a larger body size, who are more responsive, yet females raised in isolation are also more responsive. This contradictory result could potentially be attributed to the fact that female preference is dependent on multiple environmental factors which can interact to direct mate choice decisions (Rosenthal 2015). Females reared in the high density environment that are larger and more responsive may be driven by a reduction in mating costs due to favourable physical conditions, while females raised in isolation are mainly driven by a lack of social experience indicating that mating opportunities will be low, and thus the cost of being less responsive is higher.

The effect of a faster development time leading to larger body sizes in both females and males may also be examined within the framework of the pace-of-life syndrome hypothesis (POLS), where individuals with a "fast" pace of life are expected to exhibit a "live fast-die young" strategy (Ricklefs \& Wikelski 2002). These individuals develop faster, reproduce earlier, exhibit more risk prone behaviour, and die sooner than individuals following a "slow" strategy, where you see slow development, delayed reproduction, and higher survival rates (Royauté et al. 2018). Under this hypothesis, males and females reared in the High density acoustic social environment would ascribe to the "fast pace" and those reared in the None environment would be considered "slow." 
While behaviour in both males and females was correlated with body size, and indirectly with development time, I did not find any significant differences in either juvenile or adult mortality between acoustic environmental treatments, indicating that at least until approximately 14 days post adult eclosion, mortality cannot be considered as a trade-off to growth or reproduction. Furthermore, a main assertion of the POLS is that those individuals who live fast also display riskier behaviours, such as increased mate searching or aggression. While I did find an increase in both responsiveness in females and aggression in males related to larger body sizes, I did not find an increase related to the High density acoustic rearing environment. Thus, there is no strong evidence for the POLS following exposure to acoustic social environments during development. However, a full test the POLS hypothesis would require examination of adult male and female risk taking behaviour, how they invest in their reproductive tissue, and quantifying their longevity using a survival analysis.

Collectively, my findings reveal that the acoustic social environment experienced during development can influence life history traits of both males and females and affect adult behaviour (either directly or indirectly). The differences between the sexes (male behaviour is generally affected indirectly, female behaviour is affected both directly and indirectly) are interesting as they hint towards fundamental differences social anticipatory plasticity between males and females. One potential reason for this difference could be stronger selection for females to use acoustic social information during development and during adulthood. Although sexual dimorphism in insect hearing structures is rare (Robinson \& Hall 2002) there is some evidence to suggest that, at least as adults, male and female differences do exist. For example, Bateman \& Fleming (2006), found that 
virgin females are less likely to autotomize (remove) their front legs under duress compared to mated females. The authors theorized that this was due to having the tympanic membrane located on the forelimb and its importance in phono location of mates. While males also autotomized their hind limbs faster than front, there was no effect of mating status, emphasizing the importance of tympanal organs to female mating behaviour (Bateman \& Fleming 2006b). Furthermore, my findings highlight the importance of understanding how developmental plasticity possibly constrains adult behavioural plasticity and can possibly lead to erroneous conclusions about factors that are driving adult behaviour. For example, females reared in high density environments are less responsive and prefer higher chirp rates, but males reared in the same environments did not alter their signalling behaviour at adulthood, thus there is the potential for a mismatch between available male phenotypes and female mate preference.

\subsection{Influence of Anthropogenic Noise}

Recent research into the effects of anthropogenic noise on invertebrate acoustic communication is sparse but growing (Lampe et al. 2014; Orci et al. 2016; Gurule-Small \& Tinghitella 2018; Shieh et al. 2015; Schmidt et al. 2014). My thesis contributes to these studies by supporting the theory that anthropogenic noise influences life history traits and adult behaviour. Furthermore, to my knowledge, it is only the second paper to examine the effects of anthropogenic noise during development in Orthoptera. Here I found significant interactions between the developmental acoustic social environment and anthropogenic noise on development time in males and residual mass in females (Chapter 3). In females, juveniles that were reared in the social isolation (None) acoustic environment were significantly lighter at adult eclosion in the presence of anthropogenic 
noise than without it. As discussed in Chapter 3, females reared with anthropogenic may not have been able to discern a lack of signals from potential mates and therefore may not have invested more heavily in reproductive tissues or energy stores, accounting for lower residual mass. Alternatively, stress from the anthropogenic noise may have had negative effects on the females' ability to gain weight, as has been found in other species (IglesiasCarrasco et al. 2017; De Soto et al. 2013; Kight \& Swaddle 2011). In males, the presence anthropogenic noise during development resulted in a faster development time for those juveniles reared in the Low density acoustic social environment in Chapter 3, while males from Chapters 4 and 5 developed faster in the presence of anthropogenic noise if they were from the None environment. These results seem to indicate that the effects of anthropogenic noise may not be static across similar populations, but that the effects are greatest when acoustic social information is lower or absent.

While adult male aggressive behaviour was not affected by the presence of anthropogenic noise, I found near-significant effects on mate attraction signalling in males that were reared in acoustic social isolation (None environment). Adult males from the None acoustic social environment signalled more often and at higher amplitudes when anthropogenic noise was present during development. Recent research on other orthoptera indicates that exposure to anthropogenic noise during development and at adulthood can effect adult male acoustic signalling behaviour (Costello \& Symes 2014; Lampe et al. 2014). Although as discussed in Chapter 5, these findings are preliminary and mainly concern shifts in signal frequency, which I did not observe.

Female mate preferences were also affected by the presence of anthropogenic noise during development. An examination of the preference function shapes revealed 
that the preference curves for the three rearing treatments (High, Low, and None) overlapped in those females raised with anthropogenic noise. That is, the presence of anthropogenic noise seemed to lower the preference of females from the None treatment and increased preference of females reared in the High treatment. This suggests females reared with anthropogenic noise are unable to detect the social environment they are being reared in. As such, there is a mismatch between their adult social environment and their mating preferences. Additionally, there was a near significant interaction between anthropogenic noise and the artificial signals (chirp rate) on female responsiveness (net vector scores). Females reared with anthropogenic noise tended to have a curvilinear response, where they were less attracted to extreme chirp rates and more attracted to signals representative of the population mean; conversely, females reared without anthropogenic noise had more linear responses, where they were more attracted to higher chirp rates (energetically costly signals). These findings suggest that the addition of anthropogenic noise to the developmental environment results in females that are less choosy and more responsive, similar to females raised in social isolation, leading to relaxed sexual selection and helping to maintain variation of male chirp rate within the male population.

\subsection{Limitations and Future Studies}

In a review on phenotypic plasticity Forsman (2015), argues that researchers need to move away from the study of "single traits" towards a "whole organism" perspective. Here I have done that. In that same view, I would argue that my thesis simultaneously emphasizes the importance of a "whole life" perspective versus a focus on "life stages." Within both paradigms, however, there are limitations due to the nature of both research 
methodologies and practicalities. As discussed in Chapter 4, one of the main confounding factors of my study was the affects from the physical isolation of individuals due to their being kept in individual containers to reduce physical social experience. In terms of aggression, this physical social isolation had a stronger effect than the acoustic social environments. As the detrimental effects of social isolation during both development and adulthood have been documented in several species (Lihoreau et al. 2009; Hol et al. 1999; Miller et al. 2012) it should be an important consideration in studies attempting to quantify the effects of any social environment.

Another important limitation to my study is that I did not examine whether individuals were able to alter their behaviours at different points throughout their entire adult lifespan. Changes in social experience during adulthood can also impact adult behaviour. In a review that spanned several taxa, Bretman et al. (2011) showed that adult males display a variety of plastic behavioural responses in male mating strategies in response to changing socio-sexual conditions. For example, male black field crickets (Teleogryllus commodus) alter their signalling effort depending on prior mating success (Chiswell et al. 2014). Further, in the spring field cricket (Gryllus veletis) field-captured adult male contest winners are more aggressive and produce more victory displays in the presence of an audience than without (Fitzsimmons \& Bertram 2013). Females can also display a varied array of plastic behavioural responses in response to changing sociosexual conditions. For example, adult female treehoppers (Enchenopa binotata) who experience either social isolation or unattractive male signals are less selective than females who have previous experience with attractive male signals (Fowler-Finn \& Rodríguez 2012). It is therefore possible that behavioural plasticity in response to 
changing social environments can occur at any time during the adult lifespan and future studies should attempt incororpate more than one adult life point.

My research provided evidence that studies on acoustic communication in orthoptera should also consider the inclusion of anthropogenic noise. However, as discussed in Chapters 5 and 6, both adult male acoustic signalling and female phonotaxis may use more instantaneous behavioural adjustments to anthropogenic noise pollution (Gurule-Small \& Tinghitella 2018; Schmidt et al. 2014) which I did not test. There is also the possibility that animals in my study were already genetically adapted to a noisy environment. As my crickets were descendants of wild-caught G. pennsylvanicus from an urban environment, the population may have genetically diverged from more rural populations, similar to roadside and rural populations of the grasshopper Chorthippus bigltulus (Lampe et al., 2014b). It would be interested to test if populations collected from different environments responded differently to the treatments.

Furthermore, I created my acoustic soundtracks to emulate, as close as possible, natural daily variation in both male acoustic signalling patterns and anthropogenic noise disturbance. Other studies appear to have used a continuous playback set-up (Kasumovic et al. 2011; Kasumovic, et al. 2012a; Bailey \& Zuk 2008). This means that my soundtracks may have been too conservative to elicit a stronger behavioural response.

Finally, I only tested how developmental constraints influence adult behavioural flexibility using the signalling behaviour of adult males. Given the importance of the interaction between developmental plasticity and behavioural plasticity, it is paramount that future studies consider what effects are really driving adult behaviours. Extending from my thesis, future research examining male aggressive behaviour and female mate 
preference should include full-factorial experiments where developmental and adult environments are switched. Together this will allow the field of plasticity to move forward as it will simultaneously provide information using a "whole organism" and a "whole life" perspective. Only then will we have a complete understanding of how developmental and behavioural plasticity interact to influence overall fitness.

Overall my dissertation contributes to the field of behavioural ecology by highlighting how one aspect of environmental variation, the acoustic social environment experienced during development, can have important consequences on both developmental and behavioural plasticity. It also emphasizes the importance of whole life approaches to animal research to ensure that nuances between life stages as well as indirect effects are fully considered. Furthermore, my research contributes to the growing evidence of potential effects from anthropogenic noise pollution on animal development and behaviour, particularly in Orthoptera, and provides interesting avenues to explore these relationships further. 


\section{References Cited}

Adamo, S.A. \& Hoy, R.R., 1995. Agonistic behaviour in male and female field crickets, Gryllus bimaculatus, and how behavioural context influences its expression. Animal Behavoior, 49, pp.1491-1501.

Adler, F.R. \& Drew Harvell, C., 1990. Inducible defenses, phenotypic variability and biotic environments. Trends in Ecology \& Evolution, 5(12), pp.407-10.

Agrawal, A.A., 2001. Phenotypic plasticity in the interactions and evolution of species. Science, 294(5541), pp.321-6.

Alexander, R.D., 1961. Aggressiveness, territoriality, and sexual behavior in field crickets (Orthoptera : Gryllidae). Behaviour, 17(2/3), pp.130-223.

Alexander, R.D., 1962. Evolutionary change in cricket acoustical communication. Evolution, 16, pp.443-467.

Alexander, R.D., 1968. Life cycles, origins, speciation and related phenomena in crickets. The Quarterly Review of Biology, 43(1), pp.1-42.

Alexander, R.D. \& Meral, G.H., 1967. Seasonal and daily chirping cycles in the northern spring and fall field crickets, Gryllus veletis and G. pennsylvanicus. The Ohio Journal of Science, 67(1960), pp.200-209.

Anderson, C.L. \& Kasumovic, M.M., 2017. Development rate rather than social environment influences cognitive performance in Australian black field crickets, 
Teleogryllus commodus. PeerJ, 5, pp.1-18.

Andersson, M.B., 1994. Sexaul selection, New Jersy: Princton UP.

Andersson, S. et al., 2002. Multiple receivers, multiple ornaments, and a trade-off between agonistic and epigamic signaling in a widowbird. The American Naturalist, 160(5), pp.683-91.

Atema, J., 1995. Chemical signals in the marine environment: dispersal, detection, and temporal signal analysis. Proceedings of the National Academy of Sciences, 92(1), pp.62-66.

Atwell, A. \& Wagner, W.E., 2014. Female mate choice plasticity is affected by the interaction between male density and female age in a field cricket. Animal Behaviour, 98, pp.177-183.

Bailey, N.W., Gray, B. \& Zuk, M., 2010. Acoustic experience shapes alternative mating tactics and reproductive investment in male field crickets. Current Biology, 20(9), pp.845-9.

Bailey, N.W. \& Macleod, E., 2014. Socially flexible female choice and premating isolation in field crickets (Teleogryllus spp.). Journal of Evolutionary Biology, 27(1), pp.170-180.

Bailey, N.W. \& Zuk, M., 2008. Acoustic experience shapes female mate choice in field crickets. Proceedings of the Royal Society B: Biological sciences, 275(1651), 
pp.2645-50.

Bailey, N.W. \& Zuk, M., 2009. Field crickets change mating preferences using remembered social information. Biology Letters, 5, pp.449-451.

Ball, E. \& Young, D., 1974. Structure and development of the auditory system in the prothoracic leg of the cricket Teleogryllus commodus (Walker). Zeitschrift für Zellforschung und Mikroskopische Anatomie, 147(3), pp.313-324.

Ballen, C., Shine, R. \& Olsson, M., 2014. Effects of early social isolation on the behaviour and performance of juvenile lizards, chamaeleo calyptratus. Animal Behaviour, 88, pp.1-6.

Barber, J.R., Crooks, K.R. \& Fristrup, K.M., 2010. The costs of chronic noise exposure for terrestrial organisms. Trends in Ecology and Evolution, 25(3), pp.180-189.

Bateman, I. et al., 2001. The effect of road traffic on residential property values: a literature review and hedonic pricing study, Edinburgh.

Bateman, P.W. \& Fleming, P.A., 2006a. Males are selective too: mating, but not courtship, with sequential females influences choosiness in male field crickets (Gryllus bimaculatus). Behavioral Ecology and Sociobiology, 59(4), pp.577-581.

Bateman, P.W. \& Fleming, P.A., 2006b. Sex and the single (-eared) female: Leg function, limb autotomy and mating history trade-offs in field crickets (Gryllus bimaculatus). Biology Letters, 2(1), pp.33-35.

Bateman, P.W., Gilson, L.N. \& Ferguson, J.W.., 2001. Male size and sequential mate 
preference in the cricket Gryllus bimaculatus. Animal Behaviour, 61(3), pp.631-637.

Beckers, O.M. \& Schul, J., 2008. Developmental plasticity of mating calls enables acoustic communication in diverse environments. Proceedings of the Royal Society B: Biological sciences, 275(1640), pp.1243-8.

Bee, M.A. \& Swanson, E.M., 2007. Auditory masking of anuran advertisement calls by road traffic noise. Animal Behaviour, 74(6), pp.1765-1776.

Beeler, A.E., Rauter, C.M. \& Moore, A.J., 2002. Mate discrimination by females in the burying beetle Nicrophorus orbicollis: The influence of male size on attractiveness to females. Ecological Entomology, 27(1), pp.1-6.

Benjamini, Y. \& Yekutieli, D., 2001. The control of the false discovery rate in multiple testing under dependency. The Annals of Statistics, 29, pp.1165-1188.

Bergeron, P. et al., 2010. Secondary sexual characters signal fighting ability and determine social rank in Alpine ibex (Capra ibex). Behavioral Ecology and Sociobiology, 64(8), pp.1299-1307.

Bernard, M.F., 2006. Survival trade-offs between two predators-induced phenotypes in pacific treefrogs (Pseudacris regilla). Ecology, 87(2), pp.340-346.

Bertram, S.M. et al., 2004. An electronic acoustic recorder for quantifying total signaling time, duration, rate and magnitude in acoustically signaling insects. Technical Acoustics, 20, pp.1-15.

Bertram, S.M. et al., 2011. Fine- and broad-scale approaches to understanding the 
evolution of aggression in crickets. Ethology, 117(12), pp.1067-1080.

Bertram, S.M. et al., 2016. Linking mating preferences to sexually selected traits and offspring viability: good versus complementary genes hypotheses. Animal Behaviour, 119, pp.75-86.

Bertram, S.M. \& Rook, V., 2012. Relationship between condition, aggression, signaling, courtship, and egg laying in the field cricket, Gryllus assimilis. Ethology, 118(4), pp.360-372.

Berven, K.A. \& Gill, D.E., 1983. Interpreting geographic variation in life-history traits. American Zoologist, 23, pp.85-97.

Bigelow, R., 1960. Developmental rates and diapause in Acheta pennsylvanicus (Burmeister) and Acheta veletis (Orthopera:Gryllidae). Canadian Journal of Zoology, 38, pp.973-988.

Bigelow, R., 1962. Factors affecting developmental rates and diapause in field crickets. Evolution, 16(4), pp.396-406.

Bölting, S. \& Von Engelhardt, N., 2017. Effects of the social environment during adolescence on the development of social behaviour, hormones and morphology in male zebra finches (Taeniopygia guttata). Frontiers in Zoology, 14(5).

Bonenfant, C. et al., 2002. Sex- and age-dependent effects of population density on life history traits of red deer Cervus elaphus in a temperate forest. Oikos, 25(4), pp.446- 
458.

Bretman, A. et al., 2016. Effect of competitive cues on reproductive morphology and behavioral plasticity in male fruitflies. Behavioral Ecology, 27(2), pp.452-461.

Bretman, A., Gage, M.J.G. \& Chapman, T., 2011. Quick-change artists: male plastic behavioural responses to rivals. Trends in Ecology \& Evolution, 26(9), pp.467-73.

Bretman, A., Rodriguez-Munoz, R. \& Tregenza, T., 2006. Male dominance determines female egg laying rate in crickets. Biology Letters, 2(3), pp.409-411.

Briffa, M., 2008. Decisions during fights in the house cricket, Acheta domesticus: mutual or self assessment of energy, weapons and size? Animal Behaviour, 75(3), pp.10531062.

Briscoe, A.D. \& Chittka, L., 2001. The evolution of color vision in insects. Annual Review of Entomology, 46, pp.471-510.

Bunkley, J.P. et al., 2017. Anthropogenic noise changes arthropod abundances. Ecology and Evolution, 7(9), pp.2977-2985.

Burk, T., 1988. Acoustic signals, arms races and the costs of honest signalling. The Florida Entomologist, 71(4), pp.400-409.

Cade, W. \& Cade, E., 1992. Male mating success, calling and searching behaviour at high and low densities in the field cricket, Gryllus integer. Animal Behaviour, 43(1), pp.49-56.

Cade, W.H., 1981. Field cricket spacing, and the phonotaxis of crickets and parasitoid 
flies to clumped and isolated cricket songs. Zeitschrift für Tierpsychologie, 55(4), pp.365-375.

Cade, W.H. \& Wyatt, D.R., 1984. Factors affecting calling behaviour in field crickets, Teleogryllus and Gryllus (age, weight, density, and parasites). Behaviour, 88(1/2), pp.61-75.

Candolin, U., 2003. The use of multiple cues in mate choice. Biological Reviews, 78(4), pp.575-595.

Carrière, Y. \& Roff, D.A., 1995. The evolution of offspring size and number: a test of the Smith-Fretwell model in three species of crickets. Oecologia, 102(3), pp.389-396.

Cattell, R.B., 1966. The scree test for the number of factors. Multivariate Behavioral Research, 1(2), pp.245-276.

Chiswell, R. et al., 2014. Prior mating success can affect allocation towards future sexual signaling in crickets. PeerJ, 2, p.e657.

Clémencet, J. \& Doums, C., 2007. Habitat-related microgeographic variation of worker size and colony size in the ant Cataglyphis cursor. Oecologia, 152(2), pp.211-218.

Cocroft, R.B., Rodríguez, R.L. \& Rodriguez, R.L., 2005. The behavioral ecology of insect vibratory communication. BioScience, 55(4), pp.323-334.

Conroy, L.P. \& Roff, D.A., 2018. Adult social environment alters female reproductive investment in the cricket Gryllus firmus M. Taborsky, ed. Behavioral Ecology, 
29(2), pp.440-447.

Cory, A.-L. \& Schneider, J.M., 2017. Effects of social information on life history and mating tactics of males in the orb-web spider Argiope bruennichi. Ecology and Evolution, 8, pp.344-355.

Costello, R.A. \& Symes, L.B., 2014. Effects of anthropogenic noise on male signalling behaviour and female phonotaxis in Oecanthus tree crickets. Animal Behaviour, 95, pp.15-22.

Cotton, S., Small, J. \& Pomiankowski, A., 2006. Sexual selection and conditiondependent mate preferences. Current Biology, 16(17), pp.755-765.

Crawley, J.N., Schleidt, W.M. \& Contrera, J.F., 1975. Does social environment decrease propensity to fight in male mice? Behavioral Biology, 15(1), pp.73-83.

Danchin, E. et al., 2004. Public information: from nosy neighbors to cultural evolution. Science, 305(5683), pp.487-91.

Darwin, C., 1871. The descent of man and selection in relation to sex, London, UK: Penguin.

Day, T. \& McPhail, J.D., 1996. The effect of behavioural and morphological plasticity on foraging efficiency in the threespine stickleback (Gasterosteus sp.). Oecologia, 108, pp.380-388.

Dewitt, T.J., Sih, A. \& Wilson, D.S., 1998. Costs and limits of phenotypic plasticity. 
Trends in Ecology \& Evolution, 13(2), pp.77-81.

Diaz-Fleischer, F. \& Aluja, M., 2003. Behavioural plasticity in relation to egg and time limitation: the case of two fly species in the genus Anastrepha (Diptera:

Tephritidae). Oikos, 100, pp.125-133.

Diaz, M., Parra, a. \& Gallardo, C., 2011. Serins respond to anthropogenic noise by increasing vocal activity. Behavioral Ecology, 22(2), pp.332-336.

Dingemanse, N.J. et al., 2012. Variation in personality and behavioural plasticity across four populations of the great tit Parus major. The Journal of Animal Ecology, 81(1), pp.116-26.

Dingemanse, N.J. \& Wolf, M., 2013. Between-individual differences in behavioural plasticity within populations: causes and consequences. Animal Behaviour, 85(5), pp.1031-1039.

DiRienzo, N., Pruitt, J.N. \& Hedrick, A. V, 2012. Juvenile exposure to acoustic sexual signals from conspecifics alters growth trajectory and an adult personality trait. Animal Behaviour, 84(4), pp.861-868.

Doherty, J.A. \& Storz, M.M., 1992. Calling song and selective phonotaxis in the field crickets, Gryllus firmus and G. pennsylvanicus (Orthoptera: Gryllidae). Journal of Insect Behavior, 5(5), pp.555-569.

Doutrelant, C. \& McGregor, P.K., 2000. Eavesdropping and mate choice in female 
fighting fish. Behaviour, 137(12), pp.1655-1669.

Doyon, J. \& Boivin, G., 2005. The effect of development time on the fitness of female Trichogramma evanescens. Journal of Insect Science, 5, p.4.

Duckworth, R.A., 2010. Evolution of personality: developmental constraints on behavioral flexibility. The Auk, 127(4), pp.752-758.

Emlen, D.J. et al., 2012. A mechanism of extreme growth and reliable signaling in sexually selected ornaments and weapons. Science, 337(6096), pp.860-864.

Endler, J.A., 1993. Some general comments on the evolution and design of animal communication systems. Philosophical Transactions of the Royal Society B: Biological Sciences, 340(1292), pp.215-225.

Fisher, R.A., 1930. The genetical theory of natural selection, Oxford: Clarendon Press.

Fitzsimmons, L.P. \& Bertram, S.M., 2013. Playing to an audience: the social environment influences aggression and victory displays. Biology letters, 9(4), p.20130449.

Forsman, A., 2015. Rethinking phenotypic plasticity and its consequences for individuals, populations and species. Heredity, 115(4), pp.276-284.

Fowler-Finn, K.D. \& Rodríguez, R.L., 2012. Experience-mediated plasticity in mate preferences: mating assurance in a variable environment. Evolution, 66(2), pp.459468.

French, B., EJ, M. \& Backus, V., 1986. Spatial distribution of calling field crickets, 
gryllus pennsylvanicus (Orthoptera: Gryllidae). The Florida Entomologist, 69(1), pp.255-257.

French, B.W. \& Cade, W.H., 1989. Sexual selection at varying population densities in male field crickets, Gryllus veletis and G. pennsylvanicus. Journal of Insect Behavior, 2(1), pp.105-121.

French, B.W. \& Cade, W.H., 1987. The timing of calling, movement, and mating in the field crickets and G. integer Gryllus veletis, G. pennsylvanicus, and G. integer. Behavioral Ecology and Sociobiology, 21(3), pp.157-162.

Friard, O. \& Gamba, M., 2016. BORIS: a free, versatile open-source event-logging software for video/audio coding and live observations. Methods in Ecology and Evolution, 7(11), pp.1325-1330.

Gage, A.R. \& Barnard, C.J., 1996. Male crickets increase sperm number in relation to competition and female size. Behavioral Ecology and Sociobiology, 38(5), pp.349353.

Gavrilets, S., Arnqvist, G. \& Friberg, U., 2001. The evolution of female mate choice by sexual conflict. Proceedings of the Royal Society B: Biological Sciences, 268(1466), pp.531-9.

Gilbert, S.F., Bosch, T.C.G. \& Ledón-Rettig, C., 2015. Eco-evo-devo: developmental symbiosis and developmental plasticity as evolutionary agents. Nature Reviews 
Genetics, 16(10), pp.611-622.

Gillooly, J.F. \& Ophir, A.G., 2010. The energetic basis of acoustic communication. Proceedings of the Royal Society B: Biological Sciences, 277(1686), pp.1325-1331.

Godin, J.-G.J. \& Briggs, S.E., 1996. Female mate choice under predation risk in the guppy. Animal Behaviour, 51(October 1994), pp.117-130.

Gordon, S.D. \& Uetz, G.W., 2011. Multimodal communication of wolf spiders on different substrates: Evidence for behavioural plasticity. Animal Behaviour, 81(2), pp.367-375.

Gray, D.A. \& Cade, W.H., 1999. Sex, death and genetic variation: natural and sexual selection on $\backslash$ c cricket song. Proceedings of the Royal Society B: Biological Sciences, 266(1420), p.707.

Greenfield, M.D., 1997. Sexual selection and the evolution of advertisement signals. In T. N. . Owings D.H., Beecher M.D., ed. Communication. Perspectives in Ethology. Springer, Boston, MA, pp. 145-177.

Guilford, T. \& Dawkins, M.S., 1991. Reciever psychology and the evolution of animal signals. Animal Behaviour, 42(June 1990), pp.1-14.

Gurule-Small, G.A. \& Tinghitella, R.M., 2018. Developmental experience with anthropogenic noise hinders adult mate location in an acoustically signalling invertebrate. Biology Letters, 14(2), p.20170714.

Gwinner, E., 1996. Circadian and circannual programmes in avian migration. The 
Journal of Experimental Biology, 199, pp.39-48.

Hack, M.A., 1997. The energetic costs of fighting in the house cricket, Acheta domesticus L. Behavioral Ecology, 8(1), pp.28-36.

Halfwerk, W. et al., 2011. Low-frequency songs lose their potency in noisy urban conditions. Proceedings of the National Academy of Sciences, 108(35), pp.1454914554.

Hanna, D. et al., 2011. Anthropogenic noise affects song structure in red-winged blackbirds (Agelaius phoeniceus). The Journal of Experimental Biology, 214, pp.3549-3556.

Harrison, S.J. et al., 2013. Calling, courtship, and condition in the fall field cricket, Gryllus pennsylvanicus. PlLS one, 8(3), p.e60356.

Harrison, S.J., Godin, J.-G.J. \& Bertram, S.M., 2017. Influence of dietary nutrient balance on aggression and signalling in male field crickets. Animal Behaviour, 134, pp.123-134.

Hartig, F., 2017. DHARMa: residual diagnostics for hierarchical (multi-level/mixed) regression models. R package version 0.1. 5 .

Hebets, E. a, 2003. Subadult experience influences adult mate choice in an arthropod: exposed female wolf spiders prefer males of a familiar phenotype. Proceedings of the National Academy of Sciences, 100(23), pp.13390-5.

Hedrick, A. V., 1986. Female preferences for male calling bout duration in a field cricket. 
Behavioral Ecology and Sociobiology, 19(1), pp.73-77.

Hedrick, A. V \& Kortet, R., 2012. Effects of body size on selectivity for mating cues in different sensory modalities. Biological Journal of the Linnean Society, 105(1), pp.160-168.

Hedwig, B. \& Poulet, J.F., 2004. Complex auditory behaviour emerges from simple reactive steering. Nature, 430, pp.781-785.

Hesse, S. \& Thünken, T., 2014. Growth and social behavior in a cichlid fish are affected by social rearing environment and kinship. Naturwissenschaften, 101(4), pp.273283.

Heuschele, J. et al., 2009. Environment-dependent use of mate choice cues in sticklebacks. Behavioral Ecology, 20(6), pp.1223-1227.

Hirtenlehner, S. \& Römer, H., 2014. Selective phonotaxis of female crickets under natural outdoor conditions. Journal of Comparative Physiology. A, 200(3), pp.23950.

Hissmann, K., 1991. Phonotaxis of male Crickets (Gryllus campestris) in a field population as an indication of territoriality (Orthoptera: Gryllidae). Journal of Insect Behavior, 4(5), pp.675-681.

Hoback, W.W. \& Wagner, W.E., 1997. The energetic cost of calling in the variable field cricket, Gryllus lineaticeps. Physiological Entomology, 22, pp.286-290.

Hofmann, H.A. \& Schildberger, K., 2001. Assessment of strength and willingness to 
fight during aggressive encounters in crickets. Animal Behaviour, 62(2), pp.337348.

Hol, T. et al., 1999. Isolation during the play period in infancy decreases adult social interactions in rats. Behavioural Brain Research, 100(1-2), pp.91-97.

Honěk, A., 1993. Intraspecific variation in body size and fecundity in insects: a general relationship. Oikos, 66(3), pp.483-492.

Huber, F. et al., 1984. Auditory behavior of the cricket - III. Tracking of male calling song by surgically and developmentally one-eared females, and the curious role of the anterior tympanum. Journal of Comparative Physiology A, 155(6), pp.725-738.

Hughes, K.A. et al., 1999. Familiarity leads to female mate preference for novel males in the guppy, Poecilia reticulata. Animal Behaviour, 58(4), pp.907-916.

Hunt, J. et al., 2004. High-quality male field crickets invest heavily in sexual display but die young. Nature, 432(7020), pp.1024-1027.

Hunt, J., Brooks, R. \& Jennions, M.D., 2005. Female mate choice as a conditiondependent life-history trait. The American Naturalist, 166(1), pp.79-92.

Iglesias-Carrasco, M., Martín, J. \& Cabido, C., 2017. Urban habitats can affect body size and body condition but not immune response in amphibians. Urban Ecosystems, 20(6), pp.1331-1338.

Jakob, E.M., Marshall, S.D. \& Uetz, G.W., 1996. Estimating fitness: a comparison of 
body condition indices. Oikos, 77(1), p.61.

Janetos, A.C., 1980. Strategies of female mate choice: a theoretical analysis. Behavioral Ecology and Sociobiology, 7, pp.107-112.

Jang, Y., Gerhardt, H.C. \& Choe, J.C., 2008. A comparative study of aggressiveness in eastern North American field cricket species (genus Gryllus). Behavioral Ecology and Sociobiology, 62(9), pp.1397-1407.

Jennings, D.J. et al., 2010. Investment in fighting in relation to body condition, age and dominance rank in the male fallow deer, Dama dama. Animal Behaviour, 79(6), pp.1293-1300.

Jennions, M.D. \& Petrie, M., 1997. Variation in mate choice and mating preferences: a review of causes and consequences. Biological Reviews, 72(2), pp.283-327.

Jirotkul, M., 1999a. Operational sex ratio influences female preference and male- male competition in guppies. Animal Behaviour, 58, pp.287-294.

Jirotkul, M., 1999b. Population density influences male - male competition in guppies. Animal Behaviour, 58, pp.1169-1175.

Johnstone, R.A., 1995. Honest advertisement of multiple qualities using multiple signals. Journal of Theoretical Biology, 177(1), pp.87-94.

Judge, K. a, Ting, J.J. \& Gwynne, D.T., 2008. Condition dependence of male life span and calling effort in a field cricket. Evolution, 62(4), pp.868-78.

Judge, K.A., 2011. Do male field crickets, Gryllus pennsylvanicus, signal their age? 
Animal Behaviour, 81, pp.185-194.

Judge, K.A., 2010. Female social experience affects the shape of sexual selection on males. Evolutionary Ecology Research, 12(3), pp.389-402.

Judge, K.A., 2008. Survival, song and sexual selection.

Judge, K.A. \& Bonanno, V.L., 2008. Male weaponry in a fighting cricket. PLoS ONE, $3(12)$.

Judge, K.A., Ting, J.J. \& Gwynne, D.T., 2014. Condition dependence of female choosiness in a field cricket. Journal of Evolutionary Biology, 27(11), pp.2529-40.

Kaiser, H.F., 1964. A method for determining eigenvalues. Journal of the Society for Industrial and Applied Mathematics, 12(1), pp.238-248.

Kasumovic, M.M. et al., 2009. Evidence for developmental plasticity in response to demographic variation in nature. Ecology, 90(8), pp.2287-2296.

Kasumovic, M.M., Hall, M.D., et al., 2012. Socially cued developmental plasticity affects condition-dependent trait expression. Behavioral Ecology, 24(2), pp.429-434.

Kasumovic, M.M. et al., 2011. The importance of listening: juvenile allocation shifts in response to acoustic cues of the social environment. Journal of Evolutionary Biology, 24(6), pp.1325-34.

Kasumovic, M.M. \& Andrade, M.C.B., 2006. Male development tracks rapidly shifting 
sexual versus natural selection pressures. Current Biology, 16(7), pp.242-243.

Kasumovic, M.M. \& Brooks, R.C., 2011. It's all who you know: the evolution of socially cued anticipatory plasticity as a mating strategy. The Quarterly Review of Biology, 86(3), pp.181-197.

Kasumovic, M.M., Hall, M.D. \& Brooks, R.C., 2012. The juvenile social environment introduces variation in the choice and expression of sexually selected traits. Ecology and Evolution, 2(5), pp.1036-47.

Katti, M. \& Warren, P.S., 2004. Tits, noise and urban bioacoustics. Trends in Ecology \& Evolution, 19(3), pp.109-10.

Kelly, C.D., Tawes, B.R. \& Worthington, A.M., 2014. Evaluating indices of body condition in two cricket species. Ecology and Evolution, 4(23), pp.4476-4487.

Kight, C.R. \& Swaddle, J.P., 2011. How and why environmental noise impacts animals: An integrative, mechanistic review. Ecology Letters, 14(10), pp.1052-1061.

Kilmer, J.T. et al., 2017. Describing mate preference functions and other function-valued traits. Journal of Evolutionary Biology, 30(9), pp.1658-1673.

Kokko, H. et al., 2003. The evolution of mate choice and mating biases. Proceedings of the Royal Society B: Biological Sciences, 270(1515), pp.653-664.

Kokko, H. \& Rankin, D.J., 2006. Lonely hearts or sex in the city? Density-dependent effects in mating systems. Philosophical Transactions of the Royal Society B 
Biological Sciences, 361(1466), pp.319-334.

Kortet, R. \& Hedrick, A., 2005. The scent of dominance: female field crickets use odour to predict the outcome of male competition. Behavioral Ecology and Sociobiology, 59(1), pp.77-83.

Kotiaho, J.S., Simmons, L.W. \& Tomkins, J.L., 2001. Towards a resolution of the lek paradox. Nature, 410(6829), pp.684-686.

Laidre, M.E. \& Johnstone, R.A., 1872. Animal signals. , pp.829-833.

Laiolo, P., 2010. The emerging significance of bioacoustics in animal species conservation. Biological Conservation, 143(7), pp.1635-1645.

Lampe, U. et al., 2012. Staying tuned: grasshoppers from noisy roadside habitats produce courtship signals with elevated frequency components S. Patek, ed. Functional Ecology, 26(6), pp.1348-1354.

Lampe, U., Reinhold, K. \& Schmoll, T., 2014. How grasshoppers respond to road noise: developmental plasticity and population differentiation in acoustic signalling S. Patek, ed. Functional Ecology, 28(3), pp.660-668.

Leather, S.R., 1988. Size, reproductive potential and fecundity in insects: things aren't as simple as they seem. Oikos, 51(3), pp.386-389.

Leimar, O., 1996. Life history plasticity: influence of photoperiod on growth and development in the common blue butterfly. Oikos, 76(2), pp.228-234.

Lengagne, T., 2008. Traffic noise affects communication behaviour in a breeding anuran, 
Hyla arborea. Biological Conservation, 141(8), pp.2023-2031.

Levins, R., 1962. Theory of fitness in a heterogeneous environment. I. The fitness set and adaptive function. The American Naturalist, 96(891), pp.361-373.

Lierheimer, V.F. \& Tinghitella, R.M., 2017. Quantity and quality of available mates alters female responsiveness but not investment in the Pacific field cricket, Teleogryllus oceanicus. Behavioral Ecology and Sociobiology, 71(5).

Lihoreau, M., Brepson, L. \& Rivault, C., 2009. The weight of the clan: even in insects, social isolation can induce a behavioural syndrome. Behavioural Processes, 82(1), pp.81-84.

Loranger, M.J. \& Bertram, S.M., 2016. The effect of male dominance on female choice in a field cricket (Gryllus assimilis). Animal Behaviour, 114, pp.45-52.

Luther, D. a. \& Derryberry, E.P., 2012. Birdsongs keep pace with city life: changes in song over time in an urban songbird affects communication. Animal Behaviour, 83(4), pp.1059-1066.

McGraw, K.J., 2008. An update on the honesty of melanin-based color signals in birds. Pigment Cell and Melanoma Research, 21(2), pp.133-138.

McGregor, P.K. \& Peake, T.M., 2000. Communication networks: social environments for receiving and signalling behaviour. Acta Ethologica, 2(2), pp.71-81.

Mery, F. \& Burns, J.G., 2010. Behavioural plasticity: an interaction between evolution 
and experience. Evolutionary Ecology, 24(3), pp.571-583.

Miller, C.W. et al., 2012. Natal social environment influences habitat selection later in life. Animal Behaviour, 83(2), pp.473-477.

Milner, R.N.C., Jennions, M.D. \& Backwell, P.R.Y., 2008. Does the environmental context of a signalling male influence his attractiveness? Animal Behaviour, 76(5), pp.1565-1570.

Mitchell, G.D. et al., 1966. Long-term effects of total social isolation upon behavior of rhesus monkeys. Psychological Reports, 18, pp.567-580.

Mitra, C., 2011. Life history tradeoffs and phenotypic plasticity: the tale of a flight polyphenic field cricket. University of Nebraska.

Mockford, E.J. \& Marshall, R.C., 2009. Effects of urban noise on song and response behaviour in great tits. Proceedings of the Royal Society B: Biological Sciences, 276(1669), pp.2979-85.

Moczek, A.P. et al., 2011. The role of developmental plasticity in evolutionary innovation. Proceedings of the Royal Society B: Biological Sciences, 278(1719), pp.2705-2713.

Molet, M. et al., 2017. Effect of temperature and social environment on worker size in the ant Temnothorax nylanderi. Journal of Thermal Biology, 67(April), pp.22-29.

Montague, M.J., Danek-Gontard, M. \& Kunc, H.P., 2012. Phenotypic plasticity affects the response of a sexually selected trait to anthropogenic noise. Behavioral Ecology, 
24(2), pp.343-348.

Montroy, K., Loranger, M.J. \& Bertram, S.M., 2016. Male crickets adjust their aggressive behavior when a female is present. Behavioural Processes, 124, pp.108114.

Moore, A.J., Brodie III, E.D. \& Wolf, J.B., 1997. Indirect genetic effects of social interactions. Evolution, 51(5), pp.1352-1362.

Morley, E.L., Jones, G. \& Radford, A.N., 2013. The importance of invertebrates when considering the impacts of anthropogenic noise. Proceedings of the Royal Society B: Biological Sciences, 281(1776), pp.20132683-20132683.

Morse, D.H., 1971. The foraging of warblers isolated on small island. Ecology, 52(2), pp.216-228.

Mousseau, T.A. \& Roff, D.A., 1989. Adaptation to seasonality in a cricket: patterns of phenotypic and genotypic variation in body size and diapause expression along a cline in season length. Evolution, 43(7), pp.1483-1496.

Moya-Laraño, J. et al., 2008. Analysing body condition: mass, volume or density? Journal of Animal Ecology, 77(6), pp.1099-1108.

Nagamoto, J., Aonuma, H. \& Hisada, M., 2005. Discrimination of conspecific individuals via cuticular pheromones by males of the cricket Gryllus bimaculatus. Zoological science, 22(10), pp.1079-88.

Naimi, B. et al., 2014. Where is positional uncertainty a problem for species distribution 
modelling? Ecography, 37(2), pp.191-203.

Narins, P.M., 1990. Seismic in communicati anuran amphibians white-lipped frogs thump the ground as they chirp. BioScience, 40(4), pp.268-274.

Nieh, J.C. et al., 2005. Effect of group size on the aggression strategy of an extirpating stingless bee, Trigona spinipes. Insectes Sociaux, 52(2), pp.147-154.

Niehaus, A.C. et al., 2012. Fall field crickets did not acclimate to simulated seasonal changes in temperature. Journal of Comparative Physiology. B, 182(2), pp.199-207.

Nijhout, H.F., 1999. Control mechanisms of in insects polyphenic development in polyphenic factors alter some environmental. BioScience, 49(3), pp.181-192.

Nocke, H., 1972. Physiological aspects of sound communication in crickets (Gryllus campestris L.). Journal of Comparative Physiology, 80(2), pp.141-162.

Nylin, S. \& Gotthard, K., 1998. Plasticity in life-history traits. Annual Review of Entomology, 43(125), pp.63-83.

Olvido, A. \& Mousseau, T.A., 1995. Effect of rearing environment on calling song plasticity in the striped ground cricket. Evolution, 49(6), pp.1271-1277.

Orci, K.M., Petr, K. \& Barta, K., 2016. Instantaneous song modi fi cation in response to $\mathrm{fl}$ uctuating traf fi $\mathrm{c}$ noise in the tree cricket Oecanthus pellucens. Animal Behaviour, 112, pp.187-194.

Otte, D., 1974. Effects and functions in the evolution of signaling systems. Annual 
Review of Ecology and Systematics, 5, pp.385-417.

Otter, K. et al., 1999. Do female great tits (Parus major) assess males by eavedroppings ? A field study using interactive song playback. Proceedings of the Royal Society B: Biological Sciences, 266(January), pp.1305-1309.

Pacheco, K. et al., 2013. How age influences phonotaxis in virgin female Jamaican field crickets (Gryllus assimilis). PeerJ, 1, p.e130.

Parris, K.M., Velik-lord, M. \& North, J.M. a, 2009. Frogs call at a higherpitch in traffic noise. Ecology and Society, 14(1), p.25.

Partan, S.R. \& Marler, P., 2005. Issues in the classification of multimodal communication signals. The American Naturalist, 166(2), pp.231-245.

Peake, T.M. et al., 2005. Song type matching, song type switching and eavesdropping in male great tits. Animal Behaviour, 69(5), pp.1063-1068.

Perrin, N., Petit, E.J. \& Menard, N., 2012. Social systems: demographic and genetic issues. Molecular Ecology, 21(3), pp.443-446.

Pettit-Riley, R., Estevez, I. \& Russek-Cohen, E., 2002. Effects of crowding and access to perches on aggressive behaviour in broilers. Applied Animal Behaviour Science, 79(1), pp.11-25.

Phillips, J.N. \& Derryberry, E.P., 2018. Urban sparrows respond to a sexually selected trait with increased aggression in noise. Nature, (8), pp.1-10.

Pigliucci, M., 2005. Evolution of phenotypic plasticity: where are we going now? Trends 
in Ecology \& Evolution, 20(9), pp.481-6.

Pijanowski, B.C. et al., 2011. What is soundscape ecology? An introduction and overview of an emerging new science. Landscape Ecology, 26(9), pp.1213-1232.

Pollack, G.S., 2017. Insect bioacoustics. Acoustics Today, 13(2).

Pollack, G.S. \& Hoy, R., 1981. Phonotaxis to individual rhythmic components of a complex cricket calling song. Journal of Comparative Physiology A, 144(3), pp.367-373.

Price, A. et al., 2008. Pigments, patterns, and fish behavior. Zebrafish, 5(4), pp.297-307.

Price, T. \& Schluter, D., 1991. On the low heritability of life-history traits. Evolution, 45(4), pp.856-861.

Price, T.D., Qvarnström, A. \& Irwin, D.E., 2003. The role of phenotypic plasticity in driving genetic evolution. Proceedings of the Royal Society B: Biological Sciences, 270(1523), pp.1433-40.

Rabin, L.A. et al., 2003. Anthropogenic noise and its effect on animal communication: an interface between comparative psychology and conservation biology. International Journal of Comparative Psychology, 16(2), pp.171-192.

Radford, a. N., Kerridge, E. \& Simpson, S.D., 2014. Acoustic communication in a noisy world: can fish compete with anthropogenic noise? Behavioral Ecology, 25, pp.1022-1030.

Rantala, M.J. \& Kortet, R., 2004. Male dominance and immunocompetence in a field 
cricket. Behavioral Ecology, 15(2), pp.187-191.

Rantala, M.J. \& Roff, D.A., 2005. An analysis of trade-offs in immune function, body size and development time in the Mediterranean Field Cricket, Gryllus bimaculatus. Functional Ecology, 19(2), pp.323-330.

Reaney, L.T. et al., 2011. The role of body size and fighting experience in predicting contest behaviour in the black field cricket. Behavioral Ecology and Sociobiology, 65(2), pp.217-225.

Rebar, D., Barbosa, F. \& Greenfield, M.D., 2016. Acoustic experience influences male and female pre-and postcopulatory behaviors in a bushcricket. Behavioral Ecology, 27(2), pp.434-443.

Reifer, M.L., Harrison, S.J. \& Bertram, S.M., 2018. How dietary protein and carbohydrate influence field cricket development, size, and mate attraction signalling. Animal Behaviour, In Press, pp.137-146.

Ricklefs R.E., \& Wikelski, M. (2002) The physiology/life-history nexus. Trends in Ecology and Evolution 17. pp 462-468

Rillich, J., Schildberger, K. \& Stevenson, P. a., 2007. Assessment strategy of fighting crickets revealed by manipulating information exchange. Animal Behaviour, 74(4), pp.823-836.

Robinson, D.J. \& Hall, M.J., 2002. Sound signalling in orthoptera. , 29, pp.151-278.

Rodd, F.H., Reznick, D.N. \& Sokolowski, M.B., 1997. Phenotypic plasticity in the life 
history traits of guppies: repsonses to social environment. Ecology, 78(2), pp.419433.

Rodríguez, R.L., Sullivan, L.E. \& Cocroft, R.B., 2007. Vibrational communication and reproductive isolation in the Enchenopa Binotata pecies omplex of treehoppers (Hemiptera: Membracidae ). Evolution, 58(3), pp.571-578.

Rosenthal, M.F., 2015. Mating in a variable world: the implications of environmental variation for male and female mating behavior. Univrsity of Nebraska.

Rössler, W., 1992. Postembryonic development of the complex tibial organ in the foreleg of the bushcricket Ephippiger ephippiger (Orthoptera, Tettigoniidae). Cell Tissue Res, 269, pp.505-514.

Rowe, L. \& Houle, D., 1996. The lek paradox and the capture of genetic variance by condition dependent traits. Proceedings of the Royal Society B: Biological Sciences, 263(1375), pp.1415-1421.

Rowell, G.A. \& Cade, W.H., 1993. Simulation of alternative male reproductive behavior: calling and satellite behavior in field crickets. Ecological Modelling, 65(3-4), pp.265-280.

Royauté R, Berdal MA, Garrison CR, Dochtermann NA. 2018. Paceless life? A metaanalysis of the pace-of-life syndrome hypothesis. Behavioral Ecology and Sociobiology. 72(64), https://doi.org/10.1007/s00265-018-2472-z

Rutledge, J.M., Miller, A. \& Uetz, G.W., 2010. Exposure to multiple sensory cues as a 
juvenile affects adult female mate preferences in wolf spiders. Animal Behaviour, 80(3), pp.419-426.

Rutte, C., Taborsky, M. \& Brinkhof, M.W.G., 2006. What sets the odds of winning and losing? Trends in Ecology and Evolution, 21(1), pp.16-21.

Saleh, N.W., Larson, E.L. \& Harrison, R.G., 2014. Reproductive success and body size in the cricket Gryllus firmus. Journal of Insect Behavior, 27(3), pp.346-356.

Savage, K.E. et al., 2005. Male attractiveness covaries with fighting ability but not with prior fight outcome in house crickets. Behavioral Ecology, 16(1), pp.196-200.

Schausberger, P., Gratzer, M. \& Strodl, M.A., 2017. Early social isolation impairs development, mate choice and grouping behaviour of predatory mites. Animal Behaviour, 127, pp.15-21.

Scheuber, H., Jacot, A. \& Brinkhof, M.W.G., 2003. Condition dependence of a multicomponent sexual signal in the field cricket Gryllus campestris. Animal Behaviour, 65(4), pp.721-727.

Scheuber, H., Jacot, A. \& Brinkhof, M.W.G., 2004. Female preference for multiple condition-dependent components of a sexually selected signal. Proceedings of the Royal Society B: Biological Sciences, 271(August), pp.2453-2457.

Scheuber, H., Jacot, A. \& Brinkhof, M.W.G., 2003. The effect of past condition on a multicomponent sexual signal. Proceedings of the Royal Society B: Biological 
Sciences, 270(1526), pp.1779-1784.

Schluter, D., Price, T.D. \& Rowe, L., 1991. Conflicting selection pressures and life history trade-offs. Proceedings of the Royal Society B: Biological Sciences, 246(1315), pp.11-17.

Schmidt, R., Morrison, A. \& Kunc, H.P., 2014. Sexy voices - no choices: male song in noise fails to attract females. Animal Behaviour, 94, pp.55-59.

Sedinger, J.S., Flint, P.L. \& Lindberg, M.S., 1995. Environmental Influence on LifeHistory Traits : Growth, Survival, and Fecundity in Black Brant (Branta Bernicla). Ecology, 76(8), pp.2404-2414.

Shams, S. et al., 2017. Developmental social isolation affects adult behavior, social interaction, and dopamine metabolite levels in zebrafish. Developmental Psychobiology, (August), pp.1-14.

Shieh, B.-S. et al., 2012. Acoustic adaptations to anthropogenic noise in the cicada Cryptotympana takasagona Kato (Hemiptera: Cicadidae). Acta eEhologica, 15(1), pp.33-38.

Shieh, B.-S., Liang, S.-H. \& Chiu, Y.-W., 2015. Acoustic and temporal partitioning of cicada assemblages in city and mountain environments. PLoS ONE, 10(1), p.e0116794.

Shoemaker, K.L., 2003. Interactions between immunity and reproduction in the cricket, 
Gryllus texensis. Dalhousie University, Halifax.

Sih, A., Bell, A. \& Johnson, J.C., 2004. Behavioral syndromes: an ecological and evolutionary overview. Trends in Ecology \& Evolution, 19(7), pp.372-8.

Sih, A., Hanser, S.F. \& McHugh, K.A., 2009. Social network theory: new insights and issues for behavioral ecologists. Behavioral Ecology and Sociobiology, 63(7), pp.975-988.

Simmons, A.M., 2003. Perspectives and progress in animal acoustic communication. In A. M. Simmons, A. N. Popper, \& R. R. Fay, eds. Acoustic Communication. New York: Springer-Verlag, pp. 1-14.

Simmons, L., 1986. Intermale competition and mating success in the field cricket, Gryllus-Bimaculatus (Degeer). Animal Behaviour, 34(1961), pp.567-579.

Simmons, L.W., 1986. Female choice in the field cricket Gryllus bimaculatus (De Geer). Animal Behaviour, 34(5), pp.1463-1470.

Simmons, L.W., 1988. The calling song of the field cricket, Gryllus bimaculatus (de geer): constraints on transmission and its role in intermale competition and female choice. Animal Behaviour, 36(2), pp.380-394.

Sivalinghem, S. et al., 2010. Vibratory communication in the jumping spider Phidippus clarus: Polyandry, male courtship signals, and mating success. Behavioral Ecology, 21(6), pp.1308-1314.

Smith, T.B. et al., 2008. Evolutionary consequences of human disturbance in a rainforest 
bird species from Central Africa. Molecular Ecology, 17(1), pp.58-71.

Snell-Rood, E.C., 2013. An overview of the evolutionary causes and consequences of behavioural plasticity. Animal Behaviour, 85(5), pp.1004-1011.

Snijders, L. \& Naguib, M., 2017. Communication in animal social networks: a missing link? M. Naguib et al., eds., Elsevier Ltd.

Sokolovska, N., Rowe, L. \& Johansson, F., 2000. Fitness and body size in mature odonates. Ecological Entomology, 25(2), pp.239-248.

De Soto, N.A. et al., 2013. Anthropogenic noise causes body malformations and delays development in marine larvae. Scientific Reports, 3, pp.1-5.

Souroukis, K. \& Cade, W.H., 1993. Reproductive competition and selection on male traits at varying sex ratios in the field cricket, Gryllus pennsylvanicus. Behaviour, 126(1/2), pp.45-62.

Souroukis, K., Cade, W.H. \& Rowell, G., 1992. Factors that possibly influence variation in the calling song of field crickets: temperature, time, and male size, age, and wing morphology. Canadian Journal of Zoology, 70(5), pp.950-955.

Souroukis, K. \& Murray, A.-M., 1994. Female mating behavior in the field cricket, Gryllus pennsylvanicus (Orthoptera: Gryllidae) at different operational sex ratios. Journal of Insect Behavior, 8(2), pp.269-279.

Stearns, S.C., 1980. A new view of life-history evolution. Oikos, 35(2), pp.266-281.

Stevenson, P.A. \& Rillich, J., 2013. Isolation associated aggression - a consequence of 
recovery from defeat in a territorial animal. PLoS ONE, 8(9), pp.1-11.

Stockley, P. \& Seal, N.J., 2001. Plasticity in reproductive effort of male dung flies (Scatophaga stercoraria) as a response to larval density. Functional Ecology, 15(1), pp.96-102.

Swanger, E. \& Zuk, M., 2015. Cricket responses to sexual signals are influenced more by adult than juvenile experiences. Journal of Insect Behavior, (28), pp.328-337.

Symes, L.B. \& Price, T.D., 2015. Animal communication and noise,

Taborsky, B., 2017. Developmental plasticity: preparing for life in a complex world, Elsevier Ltd.

Theebe, M.A.J., 2004. Planes, trains, and automobiles: the impact of traffic noise on house prices. The Journal of Real Estate Finance and Economics, 28(2/3), pp.209234.

Tinghitella, R.M., 2008. Rapid evolutionary change in a sexual signal: Genetic control of the mutation "flatwing" that renders male field crickets (Teleogryllus oceanicus) mute. Heredity, 100(3), pp.261-267.

Tolle, A.E. \& Wagner, W.E.J., 2010. Costly signals in a field cricket can indicate high- or low-quality direct benefits depending upon the environment. Evolution, 65(1), pp.283-294.

Tomkins, J.L. et al., 2004. Genic capture and resolving the lek paradox. Trends in 
Ecology and Evolution, 19(6), pp.323-328.

Toth, M. et al., 2011. Post-weaning social isolation induces abnormal forms of aggression in conjunction with increased glucocorticoid and autonomic stress responses. Hormones and Behavior, 60(1), pp.28-36.

Tremmel, M. \& Muller, C., 2013. Insect personality depends on environmental conditions. Behavioral Ecology, 24(2), pp.386-392.

Tschirren, B. et al., 2009. Short- and long-term consequences of early developmental conditions: A case study on wild and domesticated zebra finches. Journal of Evolutionary Biology, 22(2), pp.387-395.

Via, S. \& Lande, R., 1985. Genotype-environment interaction and the evolution of phenotypic plasticity. Evolution, 39(3), pp.505-522.

Visanuvimol, L. \& Bertram, S.M., 2011. How dietary phosphorus availability during development influences condition and life history traits of the cricket, Acheta domesticas. Journal of Insect Science, 11(63), pp.1-17.

Wagner Jr., W.E. \& Reiser, M.G., 2000. The importance of calling song and courtship song in female mate choice in the variable field cricket. Animal Behaviour, 59(6), pp.1219-1226.

Wagner Jr, W.E. \& Wyatt Hoback, W., 1999. Nutritional effects on male calling behaviour in the variable field cricket. Animal Behaviour, 57, pp.89-95.

Wagner, W.E., Kelley, R.J., Tucker, K.R., \& Harper, C.J. 2001a. Females recieve a life- 
span benefit from male ejaculates in a field cricket. Evolution, 55(5), pp.994-1001.

Wagner, W.E., 1996. Convergent song preferences between female field crickets and acoustically orienting parasitoid flies. Behavioral Ecology, 7(3), pp.279-285.

Wagner, W.E.J., Smeds, M.R. \& Wiegmann, D.D., 2001b. Experience affects female responses to male song in the variable field cricket Gryllus lineaticeps (Orthoptera, Gryllidae). Ethology, 776, pp.769-776.

Walikonis, R. et al., 1991. Attractiveness of the male Acheta domesticus calling song to females - III. The relation of age-correlated changes in syllable period recognition and phonotactic threshold to juvenile hormone III biosynthesis. Journal of Comparative Physiology A, 169(6), pp.751-764.

Walker, T.J., 1962. Factors responsible for intraspecific variation in the calling songs of crickets. Evolution, 16(4), pp.407-428.

Warren, P.S. et al., 2006. Urban bioacoustics: it's not just noise. Animal Behaviour, 71(3), pp.491-502.

Weatherhead, P.J. \& Robertson, R.J., 1979. Offspring quality and the polygyny threshold: "the sexy son hypothesis." The American Naturalist, 113(2), pp.201-208.

Webb, K.L. \& Roff, D.A., 1992. The quantitative genetics of sound production in Gryllus firmus. Animal Behaviour, 44(5), pp.823-832.

West-Eberhard, M.J., 2003. Developmental Plasticity and Evolution, New York: Oxford 
University Press.

West-Eberhard, M.J., 2005. Developmental plasticity and the origin of species differences. Proceedings of the National Academy of Sciences, 102 Suppl(2), pp.6543-6549.

West-Eberhard, M.J., 1989. Phenotyypic plasticity and the origins of diversity. Annual Review of Ecology and Systematics, 20, pp.249-278.

Whattam, E.M. \& Bertram, S.M., 2011. Effects of juvenile and adult condition on longdistance call components in the Jamaican field cricket, Gryllus assimilis. Animal Behaviour, 81(1), pp.135-144.

Whitman, D. \& Agrawal, A., 2009. What is phenotypic plasticity and why is it important? Phenotypic Plasticity of Insects.

Whitman, D.W., 2008. The significance of body size in the Orthoptera: a review. Journal of Orthoptera Research2, 17(2), pp.117-134.

Wiley, R.H., 1983. The evolution of communication: information and manipulation. Volume 2:Communication, pp.156-189.

Williams, J.R., Catania, K.C. \& Carter, C.S., 1992. Development of partner preferences in female prairie voles (Microtus ochrogaster): The role of social and sexual experience. Hormones and Behavior, 26(3), pp.339-349.

Wood, W.E. \& Yezerinac, S.M., 2006. Song sparrow (Melospiza melodia) song varies 
with urban noise. The Auk, 123(3), pp.650-659.

Yager, D.D., 1999. Structure, development, and evolution of insect auditory systems. Microscopy Research and Technique, 47(6), pp.380-400.

Zahavi, A., 1975. Mate selection-a selection for a handicap. Journal of Theoretical Biology, 53(1), pp.205-214.

Zajitschek, F. et al., 2009. Effects of juvenile and adult diet on ageing and reproductive effort of male and female black field crickets, Teleogryllus commodus. Functional Ecology, 23(3), pp.602-611. 


\section{Appendices}

\section{Appendix 1: Parameter Estimate Tables for Chapter 3}

Table A1.1. Parameter estimates for linear models examining effects of acoustic treatment on development time measures, adult body size (PC1 size) and adult residual mass in females. Significant effects are bolded, FDR $\mathrm{B}_{\mathrm{B}-\mathrm{Y}}$ corrected $P=0.02(\mathrm{~N}=285)$.

\begin{tabular}{|c|c|c|c|c|c|}
\hline Model & Parameter & Estimate & SE & $\mathbf{t}$ & $\boldsymbol{P}$ \\
\hline Hatching to Wing pad & Intercept & 32.83 & 0.28 & 116.9 & $<.0001$ \\
\hline \multirow[t]{8}{*}{ Eclosion } & Season[Fall] & 2.08 & 0.49 & 4.25 & $<.0001$ \\
\hline & Season[Spring] & -1.11 & 0.47 & -2.37 & 0.02 \\
\hline & Season[Summer] & 0.02 & 0.56 & 0.03 & 1.00 \\
\hline & Signalling Density[High] & -1.07 & 0.37 & -2.93 & $\mathbf{0 . 0 0 3 7}$ \\
\hline & Signalling Density[Low] & -0.84 & 0.37 & -2.31 & 0.0218 \\
\hline & Anthropogenic Noise[No] & -0.16 & 0.26 & -0.59 & 0.55 \\
\hline & Signalling Density[High]*Anthropogenic Noise[No] & 0.62 & 0.37 & 1.68 & 0.09 \\
\hline & Signalling Density[Low]*Anthropogenic Noise[No] & -0.45 & 0.37 & -1.22 & 0.23 \\
\hline \multirow[t]{9}{*}{ Wing pad to Adult eclosion } & Intercept & 16.47 & 0.13 & 129.90 & $<.0001$ \\
\hline & Season[Fall] & 0.01 & 0.22 & 0.43 & 0.67 \\
\hline & Season[Spring] & -0.69 & 0.21 & -3.27 & 0.0012 \\
\hline & Season[Summer] & 0.94 & 0.26 & 3.7 & 0.0003 \\
\hline & Signalling Density[High] & -0.81 & 0.17 & -4.89 & $<.0001$ \\
\hline & Signalling Density[Low] & -0.40 & 0.17 & -2.44 & $\mathbf{0 . 0 1 5 3}$ \\
\hline & Anthropogenic Noise[No] & 0.021 & 0.12 & 0.18 & 0.86 \\
\hline & Signalling Density[High]*Anthropogenic Noise[No] & 0.16 & 0.17 & 0.94 & 0.35 \\
\hline & Signalling Density[Low]*Anthropogenic Noise[No] & 0.15 & 0.17 & 0.91 & 0.36 \\
\hline
\end{tabular}




\begin{tabular}{|c|c|c|c|c|c|}
\hline \multirow[t]{9}{*}{ Hatching to Adult eclosion } & Intercept & 49.31 & 0.31 & 160.79 & $<.0001$ \\
\hline & Season[Fall] & 2.18 & 0.54 & 4.07 & $<.0001$ \\
\hline & Season[Spring] & -1.80 & 0.51 & -3.52 & 0.0005 \\
\hline & Season[Summer] & 0.96 & 0.62 & 1.56 & 0.12 \\
\hline & Signalling Density[High] & -1.88 & 0.40 & -4.70 & $<.0001$ \\
\hline & Signalling Density[Low] & -1.25 & 0.40 & -3.12 & 0.002 \\
\hline & Anthropogenic Noise[No] & -0.14 & 0.29 & -0.47 & 0.64 \\
\hline & Signalling Density[High]*Anthropogenic Noise[No] & 0.77 & 0.40 & 1.93 & 0.06 \\
\hline & Signalling Density[Low]*Anthropogenic Noise[No] & -0.29 & 0.40 & -0.74 & 0.46 \\
\hline \multirow[t]{9}{*}{ PC1 size } & Intercept & -0.03 & 0.10 & -0.32 & 0.75 \\
\hline & Season[Fall] & 0.25 & 0.18 & 1.43 & 0.15 \\
\hline & Season[Spring] & -0.30 & 0.17 & -1.75 & 0.08 \\
\hline & Season[Summer] & -0.18 & 0.20 & -0.53 & 0.60 \\
\hline & Signalling Density[High] & 0.33 & 0.13 & 2.48 & 0.0138 \\
\hline & Signalling Density[Low] & -0.18 & 0.13 & -1.35 & 0.18 \\
\hline & Anthropogenic Noise[No] & 0.07 & 0.10 & 0.73 & 0.47 \\
\hline & Signalling Density[High]*Anthropogenic Noise[No] & 0.20 & 0.13 & 1.47 & 0.14 \\
\hline & Signalling Density[Low]*Anthropogenic Noise[No] & -0.05 & 0.13 & -0.35 & 0.72 \\
\hline \multirow[t]{9}{*}{ Residual Mass } & Intercept & -0.34 & 4.05 & -0.08 & 0.93 \\
\hline & Season[Fall] & 6.90 & 7.05 & 0.98 & 0.33 \\
\hline & Season[Spring] & 3.74 & 6.74 & 0.56 & 0.58 \\
\hline & Season[Summer] & -5.24 & 8.13 & -0.64 & 0.52 \\
\hline & Signalling Density[High] & -0.31 & 5.30 & -0.06 & 0.95 \\
\hline & Signalling Density[Low] & 0.57 & 5.27 & 0.11 & 0.91 \\
\hline & Anthropogenic Noise[No] & 1.94 & 3.82 & 0.51 & 0.61 \\
\hline & Signalling Density $[\mathrm{High}]^{*}$ Anthropogenic Noise $[\mathrm{No}]$ & -9.56 & 5.31 & -1.80 & 0.07 \\
\hline & Signalling Density[Low $]^{*}$ Anthropogenic Noise $[\mathrm{No}]$ & -8.12 & 5.26 & -1.54 & 0.12 \\
\hline
\end{tabular}


Table A1.2. Parameter estimates for linear models examining effects of juvenile acoustic treatment during development on development time, body size (PC1 size) and residual mass at adulthood in males. Significant effects are bolded, FDR $\mathrm{B}_{\mathrm{Y}} \mathrm{Y}$ corrected $P=$ $0.02(\mathrm{~N}=228)$.

\begin{tabular}{|c|c|c|c|c|c|}
\hline Model & Parameter & Estimate & SE & $\mathbf{t}$ & $\boldsymbol{P}$ \\
\hline Hatching to Wing pad & Intercept & 31.37 & 0.35 & 89.42 & $<.0001$ \\
\hline \multirow[t]{8}{*}{ Eclosion } & Season[Fall] & 1.32 & 0.73 & 1.81 & 0.07 \\
\hline & Season[Spring] & -1.02 & 0.53 & -1.92 & 0.06 \\
\hline & Season[Summer] & -0.51 & 0.67 & -0.76 & 0.45 \\
\hline & Signalling Density[High] & -0.65 & 0.39 & -1.66 & 0.10 \\
\hline & Signalling Density[Low] & -0.59 & 0.40 & -1.49 & 0.14 \\
\hline & Anthropogenic Noise[No] & -0.0003 & 0.28 & 0.00 & 1.00 \\
\hline & Signalling Density[High]*Anthropogenic Noise[No] & 0.18 & 0.39 & 0.46 & 0.65 \\
\hline & Signalling Density[Low]*Anthropogenic Noise[No] & 0.26 & 0.40 & 0.65 & 0.52 \\
\hline \multirow[t]{9}{*}{ Wing pad to Adult eclosion } & Intercept & 19.53 & 0.18 & 111.33 & $<.0001$ \\
\hline & Season[Fall] & 0.11 & 0.37 & 0.31 & 0.75 \\
\hline & Season[Spring] & -0.65 & 0.27 & -2.44 & 0.02 \\
\hline & Season[Summer] & 1.14 & 0.34 & 3.39 & 0.0008 \\
\hline & Signalling Density[High] & -0.82 & 0.20 & -4.2 & $<.0001$ \\
\hline & Signalling Density[Low] & -0.29 & 0.20 & -1.49 & 0.12 \\
\hline & Anthropogenic Noise[No] & 0.12 & 0.14 & 0.88 & 0.38 \\
\hline & Signalling Density[High]*Anthropogenic Noise[No] & 0.20 & 0.20 & 0.99 & 0.32 \\
\hline & Signalling Density[Low]*Anthropogenic Noise[No] & $\mathbf{0 . 5 3}$ & 0.20 & 2.67 & 0.0081 \\
\hline \multirow[t]{3}{*}{ Hatching to Adult eclosion } & Intercept & 50.91 & 0.40 & 126.81 & $<.0001$ \\
\hline & Season[Fall] & 1.44 & 0.83 & 1.72 & 0.09 \\
\hline & Season[Spring] & -1.66 & 0.61 & -2.74 & 0.0066 \\
\hline
\end{tabular}




\begin{tabular}{|c|c|c|c|c|c|}
\hline & Season[Summer] & 0.63 & 0.77 & 0.82 & 0.42 \\
\hline & Signalling Density[High] & -1.48 & 0.45 & -3.29 & 0.0012 \\
\hline & Signalling Density[Low] & -0.89 & 0.45 & -1.96 & 0.05 \\
\hline & Anthropogenic Noise[No] & 0.12 & 0.32 & 0.38 & 0.70 \\
\hline & Signalling Density $[\mathrm{High}]^{*}$ Anthropogenic Noise $[\mathrm{No}]$ & 0.38 & 0.45 & 0.84 & 0.40 \\
\hline & Signalling Density[Low]*Anthropogenic Noise[No] & 0.79 & 0.45 & 1.74 & 0.08 \\
\hline \multirow[t]{9}{*}{ PC1 size } & Intercept & -0.04 & 0.13 & -0.33 & 0.74 \\
\hline & Season[Fall] & -0.24 & 0.28 & -0.86 & 0.40 \\
\hline & Season[Spring] & 0.16 & 0.20 & 0.82 & 0.41 \\
\hline & Season[Summer] & -0.01 & 0.25 & -0.05 & 0.96 \\
\hline & Signalling Density[High] & 0.05 & 0.15 & 0.35 & 0.73 \\
\hline & Signalling Density[Low] & 0.18 & 0.15 & 1.23 & 0.22 \\
\hline & Anthropogenic Noise[No] & -0.07 & 0.11 & -0.69 & 0.49 \\
\hline & Signalling Density $[\mathrm{High}]^{*}$ Anthropogenic Noise $[\mathrm{No}]$ & -0.18 & 0.15 & -1.21 & 0.23 \\
\hline & Signalling Density[Low]*Anthropogenic Noise[No] & -0.15 & 0.15 & -0.98 & 0.33 \\
\hline \multirow[t]{9}{*}{ Residual Mass } & Intercept & -0.33 & 2.81 & -0.12 & 0.91 \\
\hline & Season[Fall] & 1.15 & 5.84 & 0.2 & 0.85 \\
\hline & Season[Spring] & 2.71 & 4.24 & 0.64 & 0.52 \\
\hline & Season[Summer] & -2.55 & 5.38 & -0.47 & 0.64 \\
\hline & Signalling Density[High] & 1.98 & 3.14 & 0.63 & 0.53 \\
\hline & Signalling Density[Low] & -3.57 & 3.17 & -1.13 & 0.26 \\
\hline & Anthropogenic Noise[No] & -2.58 & 2.26 & -1.14 & 0.26 \\
\hline & Signalling Density[High $]^{*}$ Anthropogenic Noise[No] & -0.90 & 3.14 & -0.29 & 0.78 \\
\hline & Signalling Density[Low]*Anthropogenic Noise[No] & 5.57 & 3.17 & 1.76 & 0.08 \\
\hline
\end{tabular}


Table A1.3. Parameter estimates for the linear models examining effects of development time on body size (PC1 size) and residual mass at adulthood in females $(\mathrm{N}=285)$ males $(\mathrm{N}=228)$. Significant effects are bolded, FDR $\mathrm{B}_{\mathrm{Y}}$ corrected $P=0.027$.

\begin{tabular}{llrrrr}
\hline Model & Parameter & Estimate & SE & t & $\boldsymbol{P}$ \\
\hline Females & & & & & \\
PC1 size & Intercept & 1.29 & 0.956322 & 1.35 & 0.1779 \\
& Season[Fall] & 0.26 & 0.181439 & 1.45 & 0.1479 \\
& Season[Spring] & -0.36 & 0.173284 & -2.09 & 0.0374 \\
& Season[Summer] & -0.02 & 0.209285 & -0.11 & 0.9127 \\
& Days from Hatching to Wing pad Eclosion & 0.05 & 0.051176 & 0.9 & 0.3712 \\
& Days from Hatching to Adult Eclosion & -0.6 & 0.044645 & -1.28 & 0.2006 \\
Residual Mass & Intercept & -78.35 & 36.36009 & -2.15 & 0.032 \\
& Season[Fall] & 9.94 & 6.900264 & 1.44 & 0.1507 \\
& Season[Spring] & 8.20 & 6.587981 & 1.24 & 0.2143 \\
& Season[Summer] & -12.85 & 7.953945 & -1.62 & 0.1073 \\
& Days from Hatching to Wing pad Eclosion & $\mathbf{- 1 0 . 6 2}$ & $\mathbf{1 . 9 4 9 6 2 4}$ & $\mathbf{- 5 . 4 5}$ & $<.0001$ \\
& Days from Hatching to Adult Eclosion & $\mathbf{8 . 6 7}$ & $\mathbf{1 . 6 9 9 6 6 7}$ & $\mathbf{5 . 1}$ & $<.0001$ \\
\hline Males & & & & & \\
PC1 size & Intercept & 5.73 & 1.06 & 5.41 & $<.0001$ \\
& Season[Fall] & -0.15 & 0.26 & -0.57 & 0.57 \\
& Season[Spring] & -0.03 & 0.19 & -0.16 & 0.87 \\
& Season[Summer] & 0.20 & 0.25 & 0.83 & 0.41 \\
& Days from Hatching to Wing pad Eclosion & $\mathbf{0 . 1 6}$ & $\mathbf{0 . 0 5}$ & $\mathbf{3 . 0 7}$ & $\mathbf{0 . 0 0 2 4}$ \\
& Days from Hatching to Adult Eclosion & $\mathbf{- 0 . 2 1}$ & $\mathbf{0 . 0 4}$ & $\mathbf{- 4 . 8 5}$ & $<.0001$ \\
Residual Mass & Intercept & -87.10 & 23.16 & $\mathbf{- 3 . 7 6}$ & 0.0002 \\
& Season[Fall] & 0.86 & 5.68 & 0.15 & 0.88
\end{tabular}


Season[Spring]

Season[Summer]

Days from Hatching to Wing pad Eclosion

Days from Hatching to Adult Eclosion

\begin{tabular}{rrrr}
5.48 & 4.14 & 1.32 & 0.19 \\
-6.11 & 5.36 & -1.14 & 0.26 \\
-2.53 & 1.16 & -2.18 & 0.03 \\
$\mathbf{3 . 2 7}$ & $\mathbf{0 . 9 6}$ & $\mathbf{3 . 4 0}$ & $\mathbf{0 . 0 0 0 8}$ \\
\hline
\end{tabular}




\section{Appendix 2: Parameter Estimate Tables for Chapter 4}

Table A2.1. Parameter estimates for the linear model examining effects of acoustic treatment, weight (mg) of males before the trial,

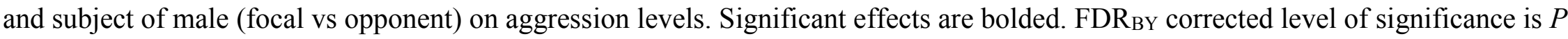
$=0.0219(\mathrm{~N}=394)$.

\begin{tabular}{llrrrr}
\hline Model & Parameter & Estimate & \multicolumn{1}{c}{ SE } & t & $\boldsymbol{P}$ \\
\hline PC1(aggression) & Intercept & -0.92 & 0.47 & -1.98 & 0.05 \\
& Season placed in Experiment[Fall] & 0.28 & 0.16 & 1.79 & 0.08 \\
& Season placed in Experiment[Spring] & -0.02 & 0.19 & -0.13 & 0.90 \\
& Season placed in Experiment[Summer] & -0.24 & 0.18 & -1.36 & 0.18 \\
& Signalling Density[High] & 0.06 & 0.13 & 0.43 & 0.67 \\
& Signalling Density[Low] & -0.12 & 0.13 & -0.92 & 0.36 \\
& Anthropogenic Noise[No] & 0.04 & 0.09 & 0.45 & 0.65 \\
& Signalling Density[High]*Anthropogenic Noise[No] & 0.05 & 0.13 & 0.37 & 0.71 \\
& Signalling Density[Low]*Anthropogenic Noise[No] & 0.01 & 0.13 & 0.08 & 0.93 \\
& Weight before Trial (mg) & 0.00 & 0.00 & 2.15 & 0.03 \\
\hline
\end{tabular}


Table A2.2. Parameter estimates for the generalized linear model on focal males examining effects of acoustic treatment, percentage difference in body weight between fighting males, body size of focal males (PC1 size), residual mass of focal males, and season on likelihood of being a winner or loser. Significant effects are bolded. FDR $\mathrm{BY}_{\mathrm{BY}}$ corrected level of significance is $P=0.0193$ (N=197).

\begin{tabular}{|c|c|c|c|c|c|c|c|}
\hline Model & Parameter & Estimate & SE & $\mathbf{X}^{2}$ & $P$ & Lower CL & Upper CL \\
\hline \multirow[t]{12}{*}{ Win/Lose } & Intercept & -1.19 & 0.22 & 39.67 & $<.0001$ & -1.65 & -0.78 \\
\hline & Season placed in Experiment[Fall] & -0.18 & 0.24 & 0.60 & 0.44 & -0.67 & 0.27 \\
\hline & Season placed in Experiment[Spring] & -0.19 & 0.28 & 0.51 & 0.48 & -0.77 & 0.32 \\
\hline & Season placed in Experiment[Summer] & 0.22 & 0.24 & 0.81 & 0.37 & -0.27 & 0.68 \\
\hline & Signalling Density[High] & -0.04 & 0.19 & 0.04 & 0.85 & -0.41 & 0.32 \\
\hline & Signalling Density[Low] & -0.06 & 0.19 & 0.11 & 0.74 & -0.44 & 0.30 \\
\hline & Anthropogenic Noise[No] & -0.06 & 0.13 & 0.23 & 0.63 & -0.31 & 0.19 \\
\hline & Signalling Density[High]*Anthropogenic Noise[No] & -0.09 & 0.19 & 0.24 & 0.62 & -0.47 & 0.27 \\
\hline & Signalling Density[Low]*Anthropogenic Noise[No] & -0.21 & 0.18 & 1.27 & 0.26 & -0.57 & 0.15 \\
\hline & Percentage in Weight Difference & 0.03 & 0.02 & 2.32 & 0.13 & -0.01 & 0.07 \\
\hline & PC1 (size) & -0.18 & 0.08 & 5.12 & 0.0237 & -0.33 & -0.02 \\
\hline & Residuals Weight at Adulthood (mg) & 0.00 & 0.00 & 1.46 & 0.23 & -0.01 & 0.00 \\
\hline
\end{tabular}


Table A2.3. Parameter estimates for the linear models on focal males examining effects of acoustic treatment, percentage difference in body weight between fighting males, body size of focal males (PC1 size), and season on aggression (PC1 aggression). Significant effects are bolded. FDR $\mathrm{BY}_{\mathrm{B}}$ corrected level of significance is $P=0.0193(\mathrm{~N}=197)$.

\begin{tabular}{llrrrr}
\hline Model & Parameter & Estimate & SE & t & $\boldsymbol{P}$ \\
\hline PC1 (aggression) & Term & 0.27 & 0.15 & 1.85 & 0.07 \\
& Intercept & 0.21 & 0.16 & 1.30 & 0.20 \\
& Season placed in Experiment[Fall] & 0.04 & 0.19 & 0.22 & 0.83 \\
& Season placed in Experiment[Spring] & -0.22 & 0.18 & -1.23 & 0.22 \\
& Season placed in Experiment[Summer] & 0.01 & 0.13 & 0.11 & 0.91 \\
& Signalling Density[High] & -0.10 & 0.13 & -0.78 & 0.44 \\
& Signalling Density[Low] & 0.02 & 0.09 & 0.28 & 0.78 \\
& Anthropogenic Noise[No] & 0.06 & 0.13 & 0.43 & 0.67 \\
& Signalling Density[High]*Anthropogenic Noise[No] & 0.02 & 0.13 & 0.16 & 0.87 \\
& Signalling Density[Low]*Anthropogenic Noise[No] & -0.03 & 0.01 & -1.76 & 0.08 \\
& Percentage in Weight Difference & $\mathbf{0 . 1 7}$ & $\mathbf{0 . 0 6}$ & $\mathbf{2 . 8 6}$ & $\mathbf{0 . 0 0 4 8}$ \\
& PC1 (size) & 0.00 & 0.00 & 1.62 & 0.11 \\
\hline
\end{tabular}


Table A2.4. Parameter estimates for the linear models examining effects of acoustic treatment, percentage difference in body weight between trial partners, body size of focal males (PC1 size), and season on aggression (PC1 aggression) in focal males for losers and winners. FDR $\mathrm{BY}_{\mathrm{BY}}$ corrected level of significance is $P=0.0193\left(\mathrm{~N}_{\text {losers }}=67, \mathrm{~N}_{\text {winners }}=130\right)$.

\begin{tabular}{llrrrr}
\hline Model & Parameter & Estimate & SE & t & $\boldsymbol{P}$ \\
\hline PC1 (aggression) & Intercept & -1.22 & 0.15 & -8.37 & $<0.0001$ \\
Losers & Season placed in Experiment[Fall] & 0.16 & 0.17 & 0.94 & 0.35 \\
& Season placed in Experiment[Spring] & -0.09 & 0.21 & -0.42 & 0.68 \\
& Season placed in Experiment[Summer] & -0.12 & 0.16 & -0.79 & 0.43 \\
& Signalling Density[High] & -0.22 & 0.12 & -1.85 & 0.07 \\
& Signalling Density[Low] & -0.18 & 0.12 & -1.47 & 0.15 \\
& Anthropogenic Noise[No] & 0.00 & 0.08 & -0.01 & 0.99 \\
& Signalling Density[High]*Anthropogenic Noise[No] & 0.08 & 0.12 & 0.64 & 0.53 \\
& Signalling Density[Low]*Anthropogenic Noise[No] & -0.17 & 0.12 & -1.39 & 0.17 \\
& Percentage in Weight Difference & -0.02 & 0.01 & -1.55 & 0.13 \\
& PC1 (size) & 0.04 & 0.06 & 0.70 & 0.49 \\
& Residuals Weight at Adulthood (mg) & 0.00 & 0.00 & 1.48 & 0.14 \\
\hline PC1 (aggression $)$ & 0.81 & 0.10 & 7.74 & $<0.0001$ \\
Winners & Intercept & -0.02 & 0.12 & -0.17 & 0.87 \\
& Season placed in Experiment[Fall] & -0.01 & 0.13 & -0.07 & 0.94 \\
& Season placed in Experiment[Spring] & -0.02 & 0.14 & -0.13 & 0.89 \\
& Season placed in Experiment[Summer] & 0.11 & 0.09 & 1.22 & 0.22 \\
& Signalling Density[High] & -0.11 & 0.10 & -1.14 & 0.26 \\
& Signalling Density[Low] & -0.05 & 0.07 & -0.73 & 0.47 \\
& Anthropogenic Noise[No] & -0.02 & 0.09 & -0.20 & 0.85 \\
& Signalling Density[High]*Anthropogenic Noise[No] & -0.08 & 0.10 & -0.81 & 0.42 \\
& Signalling Density[Low]*Anthropogenic Noise[No] & 0.00 & 0.01 & 0.36 & 0.72 \\
& Percentage in Weight Difference & 0.05 & 0.04 & 1.19 & 0.23 \\
& PC1 (size) & 0.00 & 0.00 & 1.16 & 0.25 \\
\hline & Residuals Weight at Adulthood (mg) & & & \\
& & &
\end{tabular}


Table A2.5. Parameter estimates for the linear models examining effects of acoustic treatment and season on development time, adult body size (PC1 size), residual mass at adulthood. Significant effects are bolded for both models and model effects. FDR BY $_{\text {corrected }}$ level of significance is $P=0.024(\mathrm{~N}=350)$.

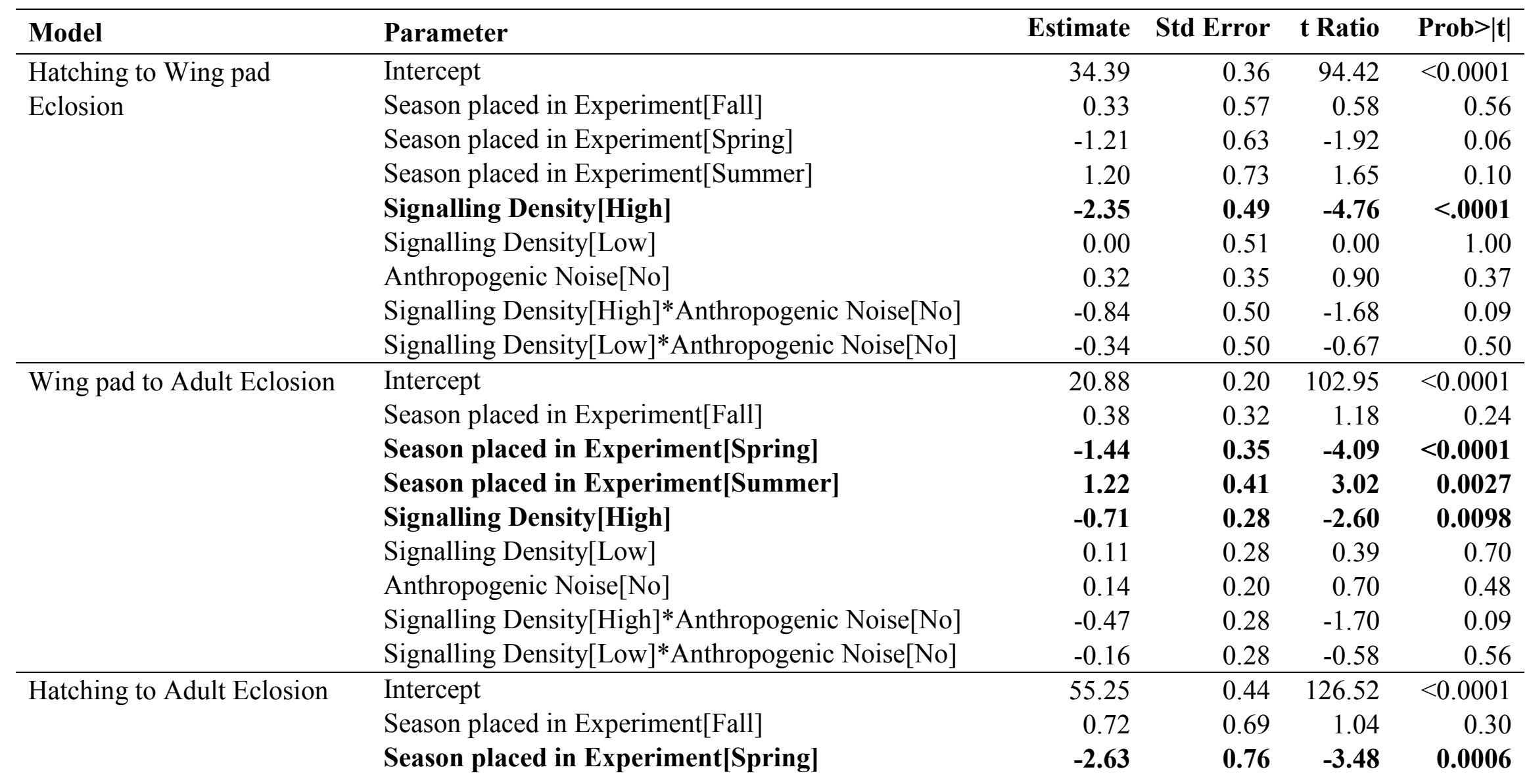




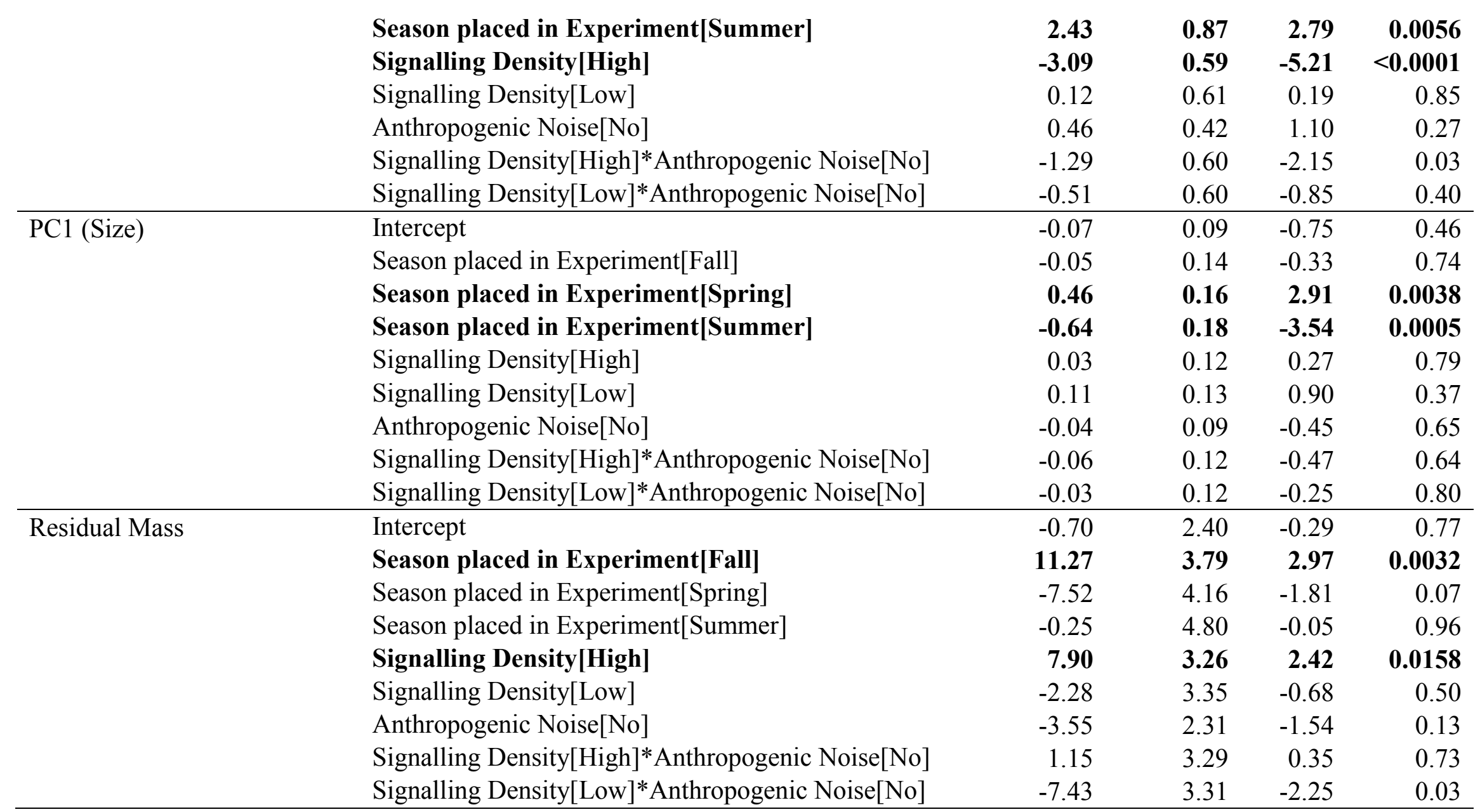

Table A2.6. Parameter estimates for the linear models examining effects of development time on body size (PC1 size) and residual

mass at adulthood in males $(\mathrm{N}=350)$. Significant effects are bolded for both models and model effects, FDR $\mathrm{BY}_{\mathrm{B}}$ corrected $P=0.027$. 


\begin{tabular}{llrrrr}
\hline Model & Model & Estimate & Std Error & t Ratio & Prob>|t| \\
\hline PC1 size & Intercept & 2.13 & 0.60 & 3.56 & 0.0004 \\
& Season placed in Experiment[Fall] & -0.03 & 0.14 & -0.22 & 0.82 \\
& Season placed in Experiment[Spring] & $\mathbf{0 . 3 4}$ & $\mathbf{0 . 1 5}$ & $\mathbf{2 . 2 6}$ & $\mathbf{0 . 0 2}$ \\
& Season placed in Experiment[Summer] & $\mathbf{- 0 . 5 2}$ & $\mathbf{0 . 1 8}$ & $\mathbf{- 2 . 9 2}$ & $\mathbf{0 . 0 0 3 7}$ \\
& Days from Hatching to Wing pad Eclosion & 0.00 & 0.01 & 0.20 & 0.84 \\
Residual Mass & Days from Wing pad to Adult Eclosion & $\mathbf{- 0 . 1 1}$ & $\mathbf{0 . 0 2}$ & $\mathbf{- 4 . 7 1}$ & $<\mathbf{0 . 0 0 0 1}$ \\
& Intercept & -17.87 & 16.67 & -1.07 & 0.28 \\
& Season placed in Experiment[Fall] & $\mathbf{1 1 . 6 4}$ & $\mathbf{3 . 8 0}$ & $\mathbf{3 . 0 6}$ & $\mathbf{0 . 0 0 2 4}$ \\
& Season placed in Experiment[Spring] & -7.24 & 4.22 & -1.72 & 0.09 \\
& Season placed in Experiment[Summer] & -1.36 & 4.93 & -0.28 & 0.78 \\
& Days from Hatching to Wing pad Eclosion & -0.03 & 0.35 & -0.08 & 0.94 \\
& Days from Wing pad to Adult Eclosion & 0.86 & 0.65 & 1.33 & 0.18 \\
\hline
\end{tabular}




\section{Appendix 3: Developmental Plasticity for Males from Chapter 4 and 5}

Male G. pennsylvanicus development time was affected by their acoustic environment, as males raised in the High density acoustic environment developed faster than males raised in either the Low density acoustic environment or males raised in social isolation (None environment). Further, males reared in isolation also developed faster in the presence of anthropogenic noise than without out (Table A2.5). Given these development patterns match Chapter 3's results, please refer to Chapter 3's discussion for details on why I believe these findings occurred. Similar to Chapter 3, rearing environment did not significantly influence body size or residual mass at adult eclosion. However, male development time from hatching to adult eclosion was influenced by a significant interaction between signalling density and anthropogenic noise. Specifically, males in the None treatment achieved their adult moult nine days sooner, on average, when there was Anthropogenic noise present. This conflicting finding provides partial support for the prediction that anthropogenic noise may interfere with juveniles' ability to accurately access the adult cohort and properly align their development to suite the expected social environment. The fact that the growth trajectories for males in this chapter are different from what I found in Chapter 3 suggests that anthropogenic noise can have multiple effects on life history traits and development, further supporting the idea of developmental "hedge-betting" - where male developmental plasticity allows for multiple life history trajectories (as discussed in Chapter 3) and indicating the need to for further study into the effects of anthropogenic noise across developmental stages. 


\section{Appendix 4: Parameter Estimate Table for Chapter 5}

Table A4.1. Parameter estimates of the linear models examining effects of acoustic treatment, adult body size (PC1 size) and residual mass, and aggression trial participation on signal factor 1, signal factor 2, time spent signalling, and amplitude plus interactions.

Significant effects are bolded. FDR ${ }_{\mathrm{BY}}$ corrected level of significance is $P=0.02$.

\begin{tabular}{|c|c|c|c|c|c|}
\hline Model & Parameter & Estimate & SE & $\mathbf{t}$ & $\boldsymbol{P}$ \\
\hline \multirow[t]{17}{*}{ Signal Factor 1} & Intercept & -0.02 & 0.08 & -0.22 & 0.83 \\
\hline & Season placed in Experiment[Fall] & 0.01 & 0.11 & 0.13 & 0.90 \\
\hline & Season placed in Experiment[Spring] & -0.22 & 0.17 & -1.30 & 0.20 \\
\hline & Season placed in Experiment[Summer] & 0.18 & 0.13 & 1.38 & 0.17 \\
\hline & Aggression Trial $(\mathrm{Y} / \mathrm{N})[\mathrm{N}]$ & 0.08 & 0.07 & 1.17 & 0.24 \\
\hline & Signalling Density[High] & 0.11 & 0.09 & 1.16 & 0.25 \\
\hline & Signalling Density[Low] & 0.05 & 0.09 & 0.53 & 0.60 \\
\hline & Aggression Trial $(\mathrm{Y} / \mathrm{N})[\mathrm{N}]^{*}$ Signalling Density[High] & 0.08 & 0.09 & 0.83 & 0.41 \\
\hline & Aggression Trial $(\mathrm{Y} / \mathrm{N})[\mathrm{N}]^{*}$ Signalling Density[Low $]$ & -0.06 & 0.09 & -0.65 & 0.52 \\
\hline & Anthropogenic Noise[No] & -0.05 & 0.07 & -0.74 & 0.46 \\
\hline & Aggression Trial $(\mathrm{Y} / \mathrm{N})[\mathrm{N}]^{*}$ Anthropogenic Noise[No] & -0.03 & 0.07 & -0.39 & 0.70 \\
\hline & Signalling Density[High]*Anthropogenic Noise[No] & 0.09 & 0.09 & 0.99 & 0.32 \\
\hline & Signalling Density[Low]*Anthropogenic Noise[No] & -0.08 & 0.09 & -0.82 & 0.42 \\
\hline & Aggression Trial $(\mathrm{Y} / \mathrm{N})[\mathrm{N}]^{*}$ Signalling Density[High $]^{*}$ Anthropogenic Noise[No] & -0.15 & 0.09 & -1.62 & 0.11 \\
\hline & Aggression Trial $(\mathrm{Y} / \mathrm{N})[\mathrm{N}]^{*}$ Signalling Density[Low]*Anthropogenic Noise[No] & 0.13 & 0.09 & 1.40 & 0.16 \\
\hline & Prin1 (Size) & 0.28 & $\mathbf{0 . 0 4}$ & 6.76 & $<0.0001$ \\
\hline & Residuals Weight at Adult Eclosion & 0.00 & 0.00 & 1.05 & 0.30 \\
\hline \multirow[t]{4}{*}{ Signal Factor 2} & Intercept & 0.00 & 0.10 & 0.00 & 1.00 \\
\hline & Season placed in Experiment[Fall] & -0.07 & 0.12 & -0.54 & 0.59 \\
\hline & Season placed in Experiment[Spring] & -0.11 & 0.19 & -0.57 & 0.57 \\
\hline & Season placed in Experiment[Summer] & 0.24 & 0.15 & 1.58 & 0.12 \\
\hline
\end{tabular}


Aggression Trial (Y/N)[N]

Signalling Density[High]

Signalling Density[Low]

Aggression Trial $(\mathrm{Y} / \mathrm{N})[\mathrm{N}] *$ Signalling Density[High]

Aggression Trial $(\mathrm{Y} / \mathrm{N})[\mathrm{N}]^{*}$ Signalling Density[Low

Anthropogenic Noise[No]

Aggression Trial $(\mathrm{Y} / \mathrm{N})[\mathrm{N}]^{*}$ Anthropogenic Noise[No]

Signalling Density[High]*Anthropogenic Noise[No]

Signalling Density[Low ${ }^{*}$ Anthropogenic Noise[No]

Aggression Trial $(\mathrm{Y} / \mathrm{N})[\mathrm{N}]^{*}$ Signalling Density[High]*Anthropogenic Noise[No]

Aggression Trial $(\mathrm{Y} / \mathrm{N})[\mathrm{N}]{ }^{*}$ Signalling Density[Low ${ }^{*}$ Anthropogenic Noise[No]

Prin1 (Size)

Residuals Weight at Adult Eclosion

\begin{tabular}{ll}
\hline Time Spent Signalling & Intercept \\
& Season placed in Experiment[Fall] \\
& Season placed in Experiment[Spring] \\
& Season placed in Experiment[Summer] \\
& Aggression Trial (Y/N)[N] \\
& Signalling Density[High] \\
& Signalling Density[Low] \\
& Aggression Trial (Y/N)[N]*Signalling Density[High] \\
& Aggression Trial (Y/N)[N]*Signalling Density[Low] \\
& Anthropogenic Noise[No] \\
& Aggression Trial $(\mathrm{Y} / \mathrm{N})[\mathrm{N}]^{*}$ Anthropogenic Noise[No] \\
& Signalling Density[High]*Anthropogenic Noise[No] \\
& Signalling Density[Low $]^{*}$ Anthropogenic Noise[No] \\
& Aggression Trial (Y/N)[N]*Signalling Density[High]*Anthropogenic Noise[No] \\
& Aggression Trial (Y/N)[N]*Signalling Density[Low]*Anthropogenic Noise[No] \\
& Prin1 $($ Size) \\
& Residuals Weight at Adult Eclosion \\
\hline Amplitude $(\mathrm{db})$ & Intercept
\end{tabular}

Season placed in Experiment[Fall]

Season placed in Experiment[Spring]

Season placed in Experiment[Summer]

Signalling Density[High]

Signalling Density[Low]

Aggression Trial $(\mathrm{Y} / \mathrm{N})[\mathrm{N}] *$ Signalling Density[High

Aggression Trial $(\mathrm{Y} / \mathrm{N})[\mathrm{N}]^{*}$ Signalling Density[Low

Anthropogenic Noise[No]

Agression Trial $(\mathrm{Y} / \mathrm{N})$

Sensity[High]*Anthropogenic Noise[No]

Aggression Trial $(\mathrm{Y} / \mathrm{N})[\mathrm{N}] *$ Signalling Density[High]*Anthropogenic Noise[No]

Prin1 (Size)

Intercept

\begin{tabular}{rrrr}
0.08 & 0.08 & 1.01 & 0.32 \\
-0.08 & 0.11 & -0.76 & 0.45 \\
0.00 & 0.11 & -0.02 & 0.98 \\
0.02 & 0.10 & 0.15 & 0.88 \\
0.04 & 0.11 & 0.39 & 0.69 \\
-0.02 & 0.08 & -0.32 & 0.75 \\
0.12 & 0.08 & 1.63 & 0.10 \\
0.11 & 0.11 & 1.03 & 0.30 \\
-0.17 & 0.11 & -1.61 & 0.11 \\
0.04 & 0.11 & 0.41 & 0.69 \\
0.06 & 0.11 & 0.53 & 0.60 \\
0.07 & 0.05 & 1.44 & 0.15 \\
0.00 & 0.00 & -0.91 & 0.37 \\
\hline 254.82 & 16.11 & 15.82 & $<0.0001$ \\
-2.66 & 20.64 & -0.13 & 0.90 \\
-29.27 & 32.09 & -0.91 & 0.36 \\
2.53 & 25.20 & 0.10 & 0.92 \\
15.66 & 13.30 & 1.18 & 0.24 \\
0.70 & 18.03 & 0.04 & 0.97 \\
-10.80 & 18.01 & -0.60 & 0.55 \\
-17.83 & 17.70 & -1.01 & 0.32 \\
13.16 & 18.06 & 0.73 & 0.47 \\
-8.39 & 12.76 & -0.66 & 0.51 \\
17.89 & 12.68 & 1.41 & 0.16 \\
14.51 & 17.72 & 0.82 & 0.41 \\
-44.04 & 18.09 & -2.43 & 0.02 \\
-3.11 & 17.84 & -0.17 & 0.86 \\
1.23 & 18.03 & 0.07 & 0.95 \\
16.62 & 7.96 & 2.09 & 0.04 \\
0.05 & 0.31 & 0.16 & 0.88 \\
\hline 53.23 & 0.81 & 65.81 & $<.0001$
\end{tabular}


Season placed in Experiment[Fall]

Season placed in Experiment[Spring]

Season placed in Experiment[Summer]

Aggression Trial $(\mathrm{Y} / \mathrm{N})[\mathrm{N}]$

Signalling Density[High]

Signalling Density[Low]

Aggression Trial $(\mathrm{Y} / \mathrm{N})[\mathrm{N}] *$ Signalling Density[High]

Aggression Trial $(\mathrm{Y} / \mathrm{N})[\mathrm{N}] *$ Signalling Density[Low]

Anthropogenic Noise[No]

Aggression Trial $(\mathrm{Y} / \mathrm{N})[\mathrm{N}]^{*}$ Anthropogenic Noise[No]

Signalling Density[High]*Anthropogenic Noise[No]

Signalling Density[Low]*Anthropogenic Noise[No]

Aggression Trial $(\mathrm{Y} / \mathrm{N})[\mathrm{N}]^{*}$ Signalling Density[High]*Anthropogenic Noise[No]

Aggression Trial $(\mathrm{Y} / \mathrm{N})[\mathrm{N}] *$ Signalling Density[Low]*Anthropogenic Noise[No]

Prin1 (Size)

Residuals Weight at Adult Eclosion

\begin{tabular}{rrrr}
0.32 & 1.04 & 0.31 & 0.76 \\
-2.15 & 1.61 & -1.34 & 0.18 \\
4.59 & 1.27 & 3.63 & 0.00 \\
0.94 & 0.67 & 1.41 & 0.16 \\
0.93 & 0.91 & 1.02 & 0.31 \\
0.39 & 0.90 & 0.43 & 0.67 \\
-0.45 & 0.89 & -0.50 & 0.62 \\
1.82 & 0.91 & 2.01 & 0.05 \\
-0.88 & 0.64 & -1.37 & 0.17 \\
-0.03 & 0.64 & -0.05 & 0.96 \\
1.18 & 0.89 & 1.33 & 0.19 \\
$\mathbf{- 2 . 3 4}$ & $\mathbf{0 . 9 1}$ & $\mathbf{- 2 . 5 7}$ & $\mathbf{0 . 0 1 0 9}$ \\
-0.62 & 0.90 & -0.69 & 0.49 \\
0.68 & 0.91 & 0.75 & 0.46 \\
$\mathbf{3 . 5 0}$ & $\mathbf{0 . 4 0}$ & $\mathbf{8 . 7 7}$ & $<\mathbf{0 . 0 0 0 1}$ \\
0.02 & 0.02 & 1.30 & 0.20 \\
\hline
\end{tabular}




\section{Appendix 5: Parameter Estimate Table for Chapter 6}

Table A5.1. Parameter estimates for the generalized linear model examining effects of acoustic treatment, residual mass, adult body

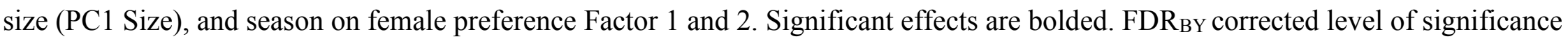
is $P=0.022$.

\begin{tabular}{llrrrr}
\hline Model & Parameter & Estimate & SE & t & $\boldsymbol{P}$ \\
\hline Preference Factor 1 & Intercept & 0.17 & 0.01 & 31.15 & $<0.0001$ \\
& SigDensLow & 0.00 & 0.01 & 0.75 & 0.46 \\
& SigDensNone & -0.01 & 0.01 & -1.68 & 0.10 \\
& AnthropYes & 0.00 & 0.00 & 0.14 & 0.89 \\
& RWeightAE & 0.00 & 0.00 & -1.30 & 0.20 \\
& PC1Size & $\mathbf{0 . 0 1}$ & $\mathbf{0 . 0 0}$ & $\mathbf{- 4 . 5 2}$ & $<\mathbf{0 . 0 0 0 1}$ \\
& SeasonSpring & $\mathbf{0 . 0 6}$ & $\mathbf{0 . 0 1}$ & $\mathbf{1 2 . 0 5}$ & $<\mathbf{0 . 0 0 0 1}$ \\
& SeasonSummer & $\mathbf{0 . 0 2}$ & $\mathbf{0 . 0 1}$ & $\mathbf{3 . 6 8}$ & $\mathbf{0 . 0 0 0 3 1}$ \\
& SeasonWinter & 0.02 & 0.01 & 2.04 & 0.04 \\
\hline Preference Factor 2 & (Intercept) & -0.16 & 0.19 & -0.85 & 0.40 \\
& SigDensLow & 0.23 & 0.18 & 1.33 & 0.18 \\
& SigDensNone & 0.39 & 0.18 & 2.24 & 0.03 \\
& AnthropYes & 0.11 & 0.14 & 0.78 & 0.44 \\
& RWeightAE & 0.00 & 0.00 & -1.03 & 0.31 \\
& PC1Size & 0.04 & 0.05 & 0.79 & 0.43 \\
& SeasonSpring & -0.15 & 0.17 & -0.87 & 0.39 \\
& SeasonSummer & -0.01 & 0.23 & -0.06 & 0.95 \\
& SeasonWinter & -0.38 & 0.31 & -1.22 & 0.22 \\
\hline
\end{tabular}


Table A5.2. Parameter estimates for the generalized linear model examining the effects of trial order, adult body size (PC1 size), residual mass, season, acoustic treatment, and chirp rate on female net vector scores. Significant effects are bolded. FDR $\mathrm{BY}_{\mathrm{B}}$ corrected level of significance is $P=0.015$.

\begin{tabular}{llrrrr}
\hline Model & Parameter & Estimate & SE & $\mathbf{t}$ & $\boldsymbol{P}$ \\
\hline Net Vector Score & Intercept & 36.75 & 4.23 & 8.69 & $<0.0001$ \\
& Order & 0.62 & 0.66 & 0.94 & 0.35 \\
& (Order (non-linear) & -0.05 & 0.04 & -1.46 & 0.14 \\
& PC1Size & $\mathbf{2 . 7 3}$ & $\mathbf{0 . 8 3}$ & $\mathbf{3 . 2 9}$ & $\mathbf{0 . 0 0 1 1 9 5}$ \\
& RMassAE & -0.01 & 0.02 & -0.73 & 0.47 \\
& SeasonSpring & -22.78 & 3.05 & -7.47 & $<\mathbf{0 . 0 0 0 1}$ \\
& SeasonSummer & $\mathbf{- 1 4 . 5 1}$ & $\mathbf{4 . 0 1}$ & $\mathbf{- 3 . 6 2}$ & $\mathbf{0 . 0 0 0 4}$ \\
& SeasonWinter & $\mathbf{- 1 6 . 0 9}$ & $\mathbf{5 . 5 0}$ & $\mathbf{- 2 . 9 2}$ & $\mathbf{0 . 0 0 4}$ \\
& SigDensLow & -3.89 & 3.12 & -1.25 & 0.21 \\
& SigDensNone & $\mathbf{1 0 . 0 6}$ & $\mathbf{3 . 1 2}$ & $\mathbf{3 . 2 3}$ & $\mathbf{0 . 0 0 1 5}$ \\
& AnthropYes & 2.38 & 2.94 & 0.81 & 0.42 \\
& Chirp Rate & $\mathbf{7 . 7 6}$ & $\mathbf{0 . 7 9}$ & $\mathbf{9 . 8 1}$ & $<\mathbf{0 . 0 0 0 1}$ \\
& Chirp Rate (non-linear) & -0.36 & 0.23 & -1.56 & 0.12 \\
& PC1Size*Chirp Rate & $\mathbf{0 . 7 4}$ & $\mathbf{0 . 2 0}$ & $\mathbf{3 . 6 3}$ & $\mathbf{0 . 0 0 0 3}$ \\
& SeasonSpring*Chirp Rate & $\mathbf{- 4 . 0 9}$ & $\mathbf{0 . 7 5}$ & $\mathbf{- 5 . 4 7}$ & $<\mathbf{0 . 0 0 0 1}$ \\
& SeasonSummer*Chirp Rate & $\mathbf{- 2 . 4 4}$ & $\mathbf{0 . 9 9}$ & $\mathbf{- 2 . 4 8}$ & $\mathbf{0 . 0 1 3}$ \\
& SeasonWinter*Chirp Rate & -3.00 & 1.32 & -2.28 & 0.02 \\
& SigDensLow*Chirp Rate & $\mathbf{- 1 . 9 6}$ & $\mathbf{0 . 7 7}$ & $\mathbf{- 2 . 5 5}$ & $\mathbf{0 . 0 1 2}$ \\
& SigDensNone*Chirp Rate & 0.42 & 0.77 & 0.54 & 0.59 \\
& AnthropYes:*Chirp Rate (non-linear) & -0.62 & 0.32 & -1.94 & 0.05 \\
\hline
\end{tabular}




\section{Appendix 6: Parameter Estimate Table for Chapter 7}

Table A6.1. Parameter estimates for the linear models examining the effects of juvenile and adult acoustic treatment, adult body size,

residual mass at adulthood and before the signalling trial, and season on signal factors 1, 2 and 3, time spent signalling, and amplitude.

Significant effects are bolded for both models and model effects. FDR $\mathrm{BY}_{\mathrm{B}}$ corrected level of significance is $P=0.018(\mathrm{~N}=118)$.

\begin{tabular}{|c|c|c|c|c|c|}
\hline Model & Parameter & Estimate & SE & $\mathbf{t}$ & $P$ \\
\hline \multirow[t]{12}{*}{ Signal Factor 1} & Intercept & 0.04 & 0.12 & 0.29 & 0.77 \\
\hline & Season[Fall] & -0.91 & 0.23 & -3.91 & 0.0002 \\
\hline & Season[Spring] & 0.07 & 0.19 & 0.39 & 0.70 \\
\hline & Season[Summer] & -0.12 & 0.15 & -0.80 & 0.43 \\
\hline & Juvenile Signalling Density[High] & 0.14 & 0.12 & 1.16 & 0.25 \\
\hline & Adult Signalling Density[High] & -0.13 & 0.09 & -1.40 & 0.16 \\
\hline & Juvenile Signalling Density[High]*Adult Signalling Density[High] & 0.05 & 0.09 & 0.56 & 0.57 \\
\hline & $\mathrm{PC}($ Size $)$ & 0.13 & 0.06 & 2.01 & 0.05 \\
\hline & Juvenile Signalling Density[High $]^{*}(\mathrm{PC}($ Size $))$ & 0.04 & 0.07 & 0.57 & 0.57 \\
\hline & Adult Signalling Density[High]*(PC(Size $))$ & -0.08 & 0.06 & -1.30 & 0.20 \\
\hline & Juvenile Signalling Density[High]*Adult Signalling Density[High]*(PC(Size $))$ & 0.11 & 0.06 & 1.80 & 0.07 \\
\hline & Residuals Mass at Adult Eclosion (mg) & -0.01 & $\mathbf{0 . 0 0}$ & -2.58 & 0.0111 \\
\hline \multirow[t]{8}{*}{ Signal Factor 2} & Intercept & 0.11 & 0.13 & 0.83 & 0.41 \\
\hline & Season[Fall] & 0.12 & 0.24 & 0.48 & 0.63 \\
\hline & Season[Spring] & 0.41 & 0.20 & 2.03 & 0.04 \\
\hline & Season [Summer] & -0.41 & 0.16 & -2.58 & 0.0111 \\
\hline & Juvenile Signalling Density[High] & 0.01 & 0.12 & 0.12 & 0.90 \\
\hline & Adult Signalling Density[High] & 0.09 & 0.10 & 0.95 & 0.34 \\
\hline & Juvenile Signalling Density[High]*Adult Signalling Density[High] & -0.17 & 0.10 & -1.78 & 0.08 \\
\hline & & & & 25 & \\
\hline
\end{tabular}




\begin{tabular}{llrrrr} 
& PC(Size) & 0.08 & 0.07 & 1.28 & 0.20 \\
& Juvenile Signalling Density[High]*(PC(Size)) & 0.06 & 0.07 & 0.91 & 0.37 \\
& Adult Signalling Density[High]*(PC(Size)) & 0.02 & 0.06 & 0.29 & 0.78 \\
& Juvenile Signalling Density[High]*Adult Signalling Density[High]*(PC(Size)) & 0.07 & 0.06 & 1.11 & 0.27 \\
& Residuals Mass at Adult Eclosion (mg) & 0.00 & 0.00 & 0.53 & 0.60 \\
\hline Signal Factor 3 & Intercept & -0.09 & 0.13 & -0.67 & 0.50 \\
& Season[Fall] & 0.16 & 0.25 & 0.63 & 0.53 \\
& Season[Spring] & 0.03 & 0.21 & 0.16 & 0.87 \\
& Season[Summer] & 0.04 & 0.17 & 0.25 & 0.80 \\
& Juvenile Signalling Density[High] & 0.18 & 0.13 & 1.43 & 0.16 \\
& Adult Signalling Density[High] & -0.05 & 0.10 & -0.50 & 0.61 \\
& Juvenile Signalling Density[High]*Adult Signalling Density[High] & -0.03 & 0.10 & -0.27 & 0.79 \\
& PC(Size) & 0.10 & 0.07 & 1.45 & 0.15 \\
& Juvenile Signalling Density[High]*(PC(Size)) & 0.05 & 0.07 & 0.75 & 0.46 \\
& Adult Signalling Density[High]*(PC(Size)) & 0.00 & 0.07 & -0.04 & 0.97 \\
& Juvenile Signalling Density[High]*Adult Signalling Density[High]*(PC(Size)) & -0.03 & 0.07 & -0.43 & 0.67 \\
& Residuals Mass at Adult Eclosion (mg) & 0.00 & 0.00 & -0.40 & 0.69 \\
\hline Time Spent & $\mathbf{2 2 0 . 1 7}$ & $\mathbf{2 2 . 4 4}$ & $\mathbf{9 . 8 1}$ & $<\mathbf{0 . 0 0 0 1}$ \\
Signalling & -32.55 & 43.36 & -0.75 & 0.45 \\
& Intercept & 22.96 & 35.71 & 0.64 & 0.52 \\
& Season[Fall] & 0.01 & 28.58 & 0.00 & 1.00 \\
& Season[Spring] & 18.01 & 21.56 & 0.84 & 0.41 \\
& Season[Summer] & -4.91 & 17.15 & -0.29 & 0.78 \\
& Juvenile Signalling Density[High] & -1.89 & 17.09 & -0.11 & 0.91 \\
& Adult Signalling Density[High] & $\mathbf{2 8 . 4 1}$ & $\mathbf{1 1 . 1 2}$ & $\mathbf{2 . 5 6}$ & $\mathbf{0 . 0 1 2}$ \\
& Juvenile Signalling Density[High]*Adult Signalling Density[High] & 8.08 & 11.58 & 0.70 & 0.49 \\
& PC(Size) & -1.71 & 10.54 & -0.16 & 0.87 \\
& Juvenile Signalling Density[High]*(PC(Size)) & 3.58 & 10.53 & 0.34 & 0.73
\end{tabular}


Residuals Mass at Adult Eclosion (mg)

$-0.97$

\begin{tabular}{rrrr}
$\mathbf{4 9 . 6 0}$ & $\mathbf{1 . 3 8}$ & $\mathbf{3 5 . 9 9}$ & $<\mathbf{0 . 0 0 0 1}$ \\
-1.83 & 2.65 & -0.69 & 0.49 \\
0.66 & 2.18 & 0.30 & 0.76 \\
-1.97 & 1.74 & -1.13 & 0.26 \\
1.63 & 1.34 & 1.22 & 0.23 \\
-0.82 & 1.07 & -0.77 & 0.44 \\
0.68 & 1.07 & 0.64 & 0.53 \\
$\mathbf{3 . 4 2}$ & $\mathbf{0 . 7 1}$ & $\mathbf{4 . 8 3}$ & $<\mathbf{0 . 0 0 0 1}$ \\
-0.16 & 0.75 & -0.22 & 0.83 \\
0.14 & 0.69 & 0.20 & 0.84 \\
-0.23 & 0.69 & -0.33 & 0.74 \\
-0.04 & 0.03 & -1.02 & 0.31 \\
\hline & & &
\end{tabular}


Table A6.2. Parameter estimates for the linear models examining the effects of acoustic treatment and season on development time, adult body size (PC1 size), residual mass at adulthood. Significant effects are bolded for both models and model effects. FDR $\mathrm{BY}$ corrected level of significance is $P=0.033(\mathrm{~N}=124)$.

\begin{tabular}{llrrrr}
\hline Model & Parameter & Estimate & SE & t & $\boldsymbol{P}$ \\
\hline Hatching to Wing pad & Intercept & 45.57 & 1.27 & 36.01 & $<0.0001$ \\
& Season[Fall] & $\mathbf{1 1 . 2 1}$ & $\mathbf{2 . 5 1}$ & $\mathbf{4 . 4 6}$ & $<\mathbf{0 . 0 0 0 1}$ \\
& Season[Spring] & $\mathbf{1 5 . 7 3}$ & $\mathbf{1 . 9 0}$ & $\mathbf{- 8 . 2 6}$ & $<\mathbf{0 . 0 0 0 1}$ \\
& Season[Summer] & 3.30 & 1.57 & 2.10 & 0.04 \\
& Juvenile Signalling Density[High] & 0.40 & 1.00 & 0.40 & 0.69 \\
\hline Wing pad to Adult Eclosion & Intercept & 24.88 & 0.67 & 37.10 & $<0.0001$ \\
& Season[Fall] & 1.04 & 1.33 & 0.78 & 0.44 \\
& Season[Spring] & $\mathbf{- 2 . 9 8}$ & $\mathbf{1 . 0 1}$ & $\mathbf{- 2 . 9 5}$ & $\mathbf{0 . 0 0 3 8}$ \\
& Season[Summer] & $\mathbf{5 . 3 2}$ & $\mathbf{0 . 8 3}$ & $\mathbf{6 . 4 0}$ & $<\mathbf{0 . 0 0 0 1}$ \\
& Juvenile Signalling Density[High] & $\mathbf{- 4 . 1 1}$ & $\mathbf{0 . 5 3}$ & $\mathbf{- 7 . 7 5}$ & $<\mathbf{0 . 0 0 0 1}$ \\
\hline \multirow{2}{*}{ Hatching to Adult Eclosion } & Intercept & 70.45 & 1.25 & 56.39 & $<0.0001$ \\
& Season[Fall] & 12.25 & 2.48 & 4.94 & $<0.0001$ \\
& Season[Spring] & $\mathbf{- 1 8 . 7 1}$ & $\mathbf{1 . 8 8}$ & $\mathbf{- 9 . 9 5}$ & $<\mathbf{0 . 0 0 0 1}$ \\
& Season[Summer] & $\mathbf{8 . 6 2}$ & $\mathbf{1 . 5 5}$ & $\mathbf{5 . 5 6}$ & $<\mathbf{0 . 0 0 0 1}$ \\
& Juvenile Signalling Density[High] & $\mathbf{- 3 . 7 1}$ & $\mathbf{0 . 9 9}$ & $\mathbf{- 3 . 7 6}$ & $\mathbf{0 . 0 0 0 3}$ \\
\hline PC1 (Size) & 0.36 & 0.20 & 1.81 & 0.07 \\
& Intercept & 0.29 & 0.39 & 0.76 & 0.45 \\
& Season[Fall] & -0.46 & 0.30 & -1.57 & 0.12 \\
& Season[Spring] & $\mathbf{- 0 . 6 3}$ & $\mathbf{0 . 2 4}$ & $\mathbf{- 2 . 5 8}$ & $\mathbf{0 . 0 1 1}$ \\
& Season[Summer] & $\mathbf{0 . 7 9}$ & $\mathbf{0 . 1 6}$ & $\mathbf{5 . 1 1}$ & $<\mathbf{0 . 0 0 0 1}$ \\
\hline Juvenile Signalling Density[High] & 0.98 & 3.78 & 0.26 & 0.80
\end{tabular}




\begin{tabular}{lrrrr} 
Season[Fall] & 4.14 & 7.50 & 0.55 & 0.58 \\
Season[Spring] & -6.72 & 5.69 & -1.18 & 0.24 \\
Season[Summer] & -1.07 & 4.69 & -0.23 & 0.82 \\
Juvenile Signalling Density[High] & $\mathbf{1 1 . 7 5}$ & $\mathbf{2 . 9 9}$ & $\mathbf{3 . 9 3}$ & $\mathbf{0 . 0 0 0 1}$ \\
\hline
\end{tabular}


